This document was prepared in conjunction with work accomplished under Contract No. DE-AC09-96SR18500 with the U. S. Department of Energy.

\title{
DISCLAIMER
}

This report was prepared as an account of work sponsored by an agency of the United States Government. Neither the United States Government nor any agency thereof, nor any of their employees, nor any of their contractors, subcontractors or their employees, makes any warranty, express or implied, or assumes any legal liability or responsibility for the accuracy, completeness, or any third party's use or the results of such use of any information, apparatus, product, or process disclosed, or represents that its use would not infringe privately owned rights. Reference herein to any specific commercial product, process, or service by trade name, trademark, manufacturer, or otherwise, does not necessarily constitute or imply its endorsement, recommendation, or favoring by the United States Government or any agency thereof or its contractors or subcontractors. The views and opinions of authors expressed herein do not necessarily state or reflect those of the United States Government or any agency thereof. 


\section{TARGETING AND ESTIMATING WASTE LOADINGS AT DWPF: A SENSITIVITY ANALYSIS}

T. B. Edwards

September 2004

Statistical Consulting Section

Savannah River National Laboratory

Aiken, SC 29808

Prepared for the U.S. Department of Energy Under Contract Number DEAC09-96SR18500

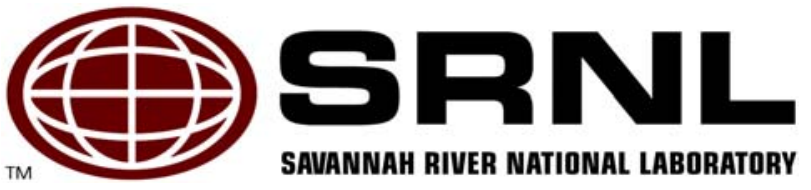


This page was intentionally left blank 
Key Words: sludge, frit, SME, Hydragard,

Retention: Permanent

\section{TARGETING AND ESTIMATING WASTE LOADINGS AT DWPF: A SENSITIVITY ANALYSIS}

T. B. Edwards

September 2004

Statistical Consulting Section

Savannah River National Laboratory

Aiken, SC 29808

Prepared for the U.S. Department of Energy Under Contract Number DEAC09-96SR18500

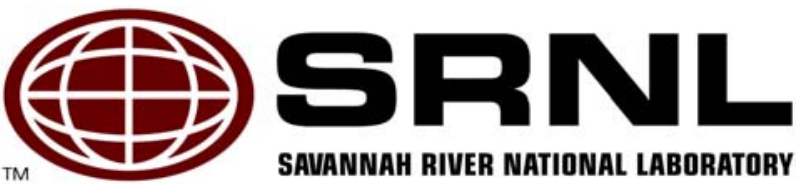




\section{EXECUTIVE SUMMARY}

Waste Loading (WL) is a measure (expressed as a percentage) of the Defense Waste Processing Facility (DWPF) glass product that comes from high level waste. In this report, the DWPF calculations used to target WL during blending decisions and those used to estimate WL during processing are investigated to assess the sensitivities of these calculations to the random uncertainties of their inputs. For the calculations used to target WLs, the uncertainties in the inputs lead to an uncertainty, at approximately $95 \%$ confidence, in the targeted WL of \pm 1.05 to \pm 1.75 percentage points depending on how the random errors in the inputs are represented. For the calculations used to estimate the WL for a given Slurry Mix Evaporator (SME) batch, the random uncertainties of the inputs to this calculation lead to an uncertainty, at approximately $95 \%$ confidence, in the estimated WL of \pm 1.50 percentage points.

Since one would expect to see agreement between the WL calculations of the targeting process and the WL calculations of the estimating process, comparisons between these WLs for SME batches 234 through 265 were conducted. The comparisons suggested that the targeted WLs and estimated WLs for these batches did not track each other as closely as would be expected based upon their random variations as outlined in this report. In an effort to reconcile the targeted and estimated WLs some issues were identified:

- During the blending process, the $\mathrm{Li}_{2} \mathrm{O}$ content planned for the next SME batch is normalized using the sum of oxides for the 16 elements being tracked (i.e., the presence of minor oxides that might account for $\sim 1$ or $2 \%$ of the SME is not accounted for). This may lead to the targeted WL being understated.

- If there is a small $(\sim 1.5 \%)$ bias in the measured $\mathrm{Li}_{2} \mathrm{O}$ content of the SME samples due to the Hydragard $\AA /$ peanut vial sampling system (as seen in a prototypical test of this system conducted by Steimke in 1995), it could have an effect on the estimated WL (the WL value as a percentage could be overstated $\sim 1 \%$ ) and to a lesser extent on the targeted WL (the targeted WL value as a percentage could be overstated $\sim 0.4 \%$ ).

- The normalization of the $\mathrm{Li}_{2} \mathrm{O}$ content of each SME sample using the sample's sum of oxides, while not suggested by the data (i.e., there does not appear to be a correlation between a low lithium recovery and a low sum of oxides for the SME samples), may actually be lessening the impact of the potential bias in the $\mathrm{Li}_{2} \mathrm{O}$ measurements for the SME samples. 


\section{TABLE OF CONTENTS}

EXECUTIVE SUMMARY iii

LIST OF FIGURES $\quad \mathrm{v}$

LIST OF TABLES $\quad \mathrm{V}$

LIST OF ACRONYMS vi

1.0 INTRODUCTION AND BACKGROUND 1

2.0 RESULTS 3

2.1 Targeting a WL for a SME batch 3

2.2 Estimating the WL attained for a SME Batch 5

2.3 Uncertainties in Estimating the WL attained for a SME Batch 7

2.4 Contrasting Targeted and Estimated Waste loadings 7

2.5 IMPACT of A potential Bias on WL's 12

3.0 CONCLUSIONS 15

4.0 REFERENCES 17

APPENDIX: Tables and Exhibits $\quad 19$ 


\section{LIST OF FIGURES}

Figure 2-1 Estimated WLs by SME Batch with 95\% Confidence Intervals Based upon 4

Samples

Figure 2-2 Estimated WLs by SME Batch with 95\% Confidence Intervals Based upon 4

Samples and a Pooled Estimate of the Standard Deviation 6

Figure 2-3 Plot of Targeted and Estimated Waste Loadings by Batch Number with

Uncertainty Bands from Sensitivity Study 8

Figure 2-4 Plot of Targeted and Estimated Waste Loadings Adjusted for 98\% Recovery by Batch Number

Figure 2-5 A Linear Fit of the WLs Calculated Using the Adjusted $\mathrm{Li}_{2} \mathrm{O}$ Values Versus the Unadjusted WLs 13

Figure 2-6 Targeted versus Estimated WLs for SME Batches 234 through 265. 14

\section{LIST OF TABLES}

Table 2-1 Simulation Results for SME Batch 261's WL Target of 32.4\% with Larger Analytical Uncertainties

Table 2-2 Simulation Results for SME Batch 261's WL Target of 32.4\% with Smaller

Analytical Uncertainties...................................................................................... 5

Table 2-3 Simulation Results for Uncertainties in Estimated WL for SME Batch 261 ........... 7

Table 2-4 Impact of the Sum of the Concentrations of the 16 Oxides Being Monitored on

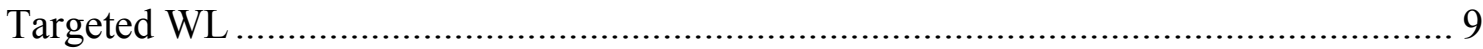

Table 2-5 Impact of the Sum of the 16 Oxides Being Monitored on Estimated WL ............. 10

Table 2-6 Impact of a $1.5 \% \mathrm{Li}_{2} \mathrm{O}$ Bias on Estimated WL 12 


\section{LIST OF ACRONYMS}

DWPF Defense Waste Processing Facility

HLW high-level waste

LCL lower confidence limit

MFT Melter Feed Tank

Prep preparation

\%RSD Percent Relative Standard Deviation

SCS Statistical Consulting Section

SME Slurry Mix Evaporator

SRAT Sludge Receipt and Adjustment Tank

SRS Savannah River Site

Std Dev Standard Deviation

TTR Technical Task Request

UCL upper confidence limit

WL Waste Loading 


\subsection{INTRODUCTION AND BACKGROUND}

The Defense Waste Processing Facility (DWPF) at the Department of Energy's Savannah River Site (SRS) in Aiken, South Carolina, has been immobilizing high-level radioactive waste (HLW) in borosilicate glass since 1996. The flowsheet for the DWPF operation involves combining each process batch of HLW sludge with an appropriate amount of glass formers called frit. The HLW and frit are combined with the heel in the Slurry Mix Evaporator (SME), and the chemical composition of this blend is determined via sampling. In fact, the SME is a hold-point for the process, and the blending and sampling of its contents are critical steps in the DWPF process control strategy.

Currently, each of the SME samples (which number at least 4) is vitrified and analyzed for its chemical composition. The resulting measurements are used by DWPF to ensure that the SME batch meets objectives of processability and product quality. Through relationships provided by glasscomposition/glass property models (e.g., those for durability, viscosity, and liquidus temperature), the product quality and processability of the SME batch are predicted, and these predictions are judged against operational constraints to ensure that the constraints are met with high confidence [1].

The same SME measurements are used to estimate the waste loading (WL) attained for the SME batch. WL is a measure of the amount (expressed as a percentage) of the glass product that comes from HLW, and the rule is the higher the WL the better, tempered by the need for the material in the SME to meet process and quality constraints and to have an acceptable melt rate (i.e., for the SME material to have no adverse impact on the DWPF's melter performance). Thus, WL and melt rate are the two important factors for achieving optimal waste throughput where waste throughput is an overall metric for how quickly a tank of HLW is processed (i.e., the faster a canister of glass is poured the better and the larger the percentage of HLW in the canister the better). Once again, the WL for a SME batch is estimated from the chemical composition measurements of the SME samples, the same samples used for the SME acceptability decision.

DWPF relies on its blending strategy (i.e., the decisions made by DWPF Process Engineering on the amount of frit to add into the SME heel along with the amount of HLW sludge transferred to the SME from the Sludge Receipt and Adjustment Tank (SRAT)) to meet the processability, product quality, and WL objectives for each SME batch. The strategy relies on measurements of the SRAT composition, of the frit composition, and of the SME heel composition as well as estimates of volumes or pounds of the additions and transfers. The measurements are used to predict the composition of the resulting SME on a glass basis. Predictions generated using the models relating glass composition to process and property models are judged against the SME acceptability criteria for the batch. Thus, the blending strategy yields a SME batch that is predicted to satisfy the process and product quality constraints as well a one that attains the WL target for the batch.

Note that the processes of targeting a WL for a batch and of estimating the WL attained for a batch both rely on measurements and as such are inherently uncertain. That is, there is uncertainty in the WL targets and estimates due to uncertainties in the measurements on which they are based. The goal of this report is to explore the sensitivities of the WL targets and estimates to uncertainties in the inputs to these important metrics of the DWPF operation. Section 2 provides the results from this study. Exploring the blending calculations employed by DWPF Process Engineering, Section 2.1 addresses the uncertainties of the targeted WL. Section 2.2 investigates the uncertainties associated with estimating the WL for a SME batch. In this section, one measure of the uncertainty in the estimated WL is obtained using the 4 SME samples. Also, in this section, a more complete uncertainty analysis is conducted by introducing 
uncertainties for the frit composition. Section 3 provides the conclusions from this study while Section 4 provides the list of references. An appendix provides supporting tables and exhibits.

The sensitivity study was initiated as part of the response to the Technical Task Request (TTR) [2] issued by DWPF Process Engineering, and the calculations and analyses were conducted using the statistical software package JMP® Version 5 [3]. 


\subsection{RESULTS}

This part of the report provides the main discussion points of the sensitivity study. In the first sub-section that follows, the calculations used to target a WL during the SME blending process are investigated, the inputs to the calculations are identified, random uncertainties for the values of these inputs are estimated, and the impact of these random uncertainties on the targeted WL is assessed. Section 2.2 explores how WL is estimated for a SME batch and information available from samples of recent SME batches (234 through 265). The variation of these data offers some insight into the uncertainty of the estimated WL for a specific SME batch. The random uncertainties of the inputs for the calculations used to estimate WL and their impact on the uncertainty of the WL values are explored in Section 2.3. Section 2.4 attempts to reconcile the targeted and estimated WLs for the SME batches in light of the uncertainties identified in the earlier sections. Finally, in Section 2.5, the impact on estimated WLs of a potential bias in the measurement of the SME samples is investigated.

\subsection{TARGETING A WL FOR A SME BATCH}

Targeting WL is part of the strategy that is utilized by DWPF Process Engineering as they plan the blending for each SME batch. Table A1 in the Appendix provides the WLs targeted by this process for batches 234 through 265 (batches produced during the processing of Sludge Batch 2 with Frit 320). Also, included in this table are the concentrations of the $\mathrm{Li}_{2} \mathrm{O}$ component of Frit 320 that were used in these blending calculations. The $\mathrm{Li}_{2} \mathrm{O}$ values are but one of the inputs used in the targeting of DWPF WLs, and the impact of the random uncertainties of these values along with the other inputs is one step along the path followed by this investigation.

All of the calculations associated with DWPF's blending process are not listed in detail as part of this report. But to provide an opportunity for the reproducibility of the results presented here, the blending for SME Batch 261 is mimicked in this report with its input values and resulting targeted WL. A JMP simulation is set up to introduce uncertainties for the inputs and to record their impacts on the resulting targeted WL. The uncertainty for each input is represented by setting up the input as a random variable that is normally distributed with a mean value equal to the nominal value for the input and a relative standard deviation representative of the uncertainty for the input. Table A2 in the Appendix provides an overview of the inputs, their nominal values, and the uncertainty (relative standard deviation) assumed for each. In this table, two views of the uncertainties are provided. In the first column of relative standard deviations, the estimates of the analytical uncertainties are taken from Edwards [4] and represent the relative standard deviation of a single measurement (i.e., $n=1$ ). In the last column of relative standard deviations, the estimates are adjusted to reflect the number of samples used to determine the average values for the inputs. For the SRAT composition information, the number of samples is 6 , and for the SME heel composition information, the number of samples is 4 . An error number is also indicated as part of the information appearing in the first column of this table. From this information, there are 11 different error sources that are being studied for the targeting phase of the WL determinations. Numbering the error sources allows for selective activation of the error sources so that their individual as well as collective impacts can be studied.

An initial investigation of the impacts of random uncertainties of the critical inputs to the WL targeting is provided in Exhibits A1 and A2 in the Appendix. These analyses are conducted with estimates of the analytical uncertainties not adjusted for the number of samples taken (i.e., all of the uncertainties are interpreted as if each of the inputs was based on a sample of size 1). This should be considered as a worst case scenario for the compositional uncertainties, since the compositions of the SRAT and the SME heel are usually based on 6 and 4 samples, respectively. Exhibit A3 in the Appendix provides an opportunity to see how the random uncertainty of each input affects the values for that input in the simulation. 
Exhibit A1 provides a plot that shows the WLs that result from 1000 perturbations (i.e., 1000 runs of the simulation) of the inputs by each error number category. The relative size of the bars in this exhibit provides insight into the comparative impacts of the error sources on WL. The $95 \%$ confidence intervals (each interval is defined by a lower confidence limit, LCL, and an upper confidence limit, UCL) for the targeted WLs from Exhibit A2 are summarized by error number in Table 2-1. Thus, with all of the probable errors activated for the worst case scenario, the $95 \%$ confidence interval for the $32.4 \%$ targeted waste loading for SME Batch 261 is given by $(30.5,34.1)$.

Table 2-1 Simulation Results for SME Batch 261's WL Target of 32.4\% with Larger Analytical Uncertainties

\begin{tabular}{||c|c|c|c|c||}
\hline $\begin{array}{c}\text { Active Error } \\
\text { Indicator }\end{array}$ & $\begin{array}{c}\text { Active Error Descriptor } \\
(\mathbf{1 0 0 0} \text { runs each) }\end{array}$ & $\begin{array}{c}\mathbf{9 5 \%} \\
\mathbf{L C L}\end{array}$ & $\begin{array}{c}\mathbf{9 5 \%} \\
\mathbf{U C L}\end{array}$ & $\begin{array}{c}\text { WL } \\
\text { Uncertainty }\end{array}$ \\
\hline 0 & All (0) & 30.6 & 34.1 & 1.75 \\
\hline 1 & Heel calcine solids (1) & 32.4 & 32.4 & 0.00 \\
\hline 2 & Heel SpG (2) & 32.4 & 32.4 & 0.00 \\
\hline 3 & Heel Chem Comps (3) & 31.1 & 33.7 & 1.30 \\
\hline 4 & Heel Volume (4) & 32.4 & 32.4 & 0.00 \\
\hline 5 & SRAT wt\% solids (5) & 31.7 & 33.1 & 0.70 \\
\hline 6 & SRAT SpG (6) & 32.1 & 32.6 & 0.25 \\
\hline 7 & SRAT Chem Comps (7) & 32.0 & 32.9 & 0.45 \\
\hline 8 & SRAT Volume (8) & 32.2 & 32.6 & 0.20 \\
\hline 9 & Frit Chem Comps (9) & 31.7 & 33.1 & 0.70 \\
\hline 10 & Frit lbs (10) & 32.2 & 32.6 & 0.20 \\
\hline 11 & Frit lbs from Can Decon (11) & 32.34 & 32.43 & 0.04 \\
\hline
\end{tabular}

Running the simulations after adjusting these uncertainties to reflect the number of samples that are typically used in determining the associated input (i.e., 4 samples for SME heel measurements and 6 for SRAT transfer measurements) leads to Exhibits A4 and A5 in the Appendix. These analyses are conducted with the estimates of the analytical uncertainties as provided in the last column of Table A2, as relative standard deviations reduced by a factor of $\sqrt{\mathrm{n}}$. Exhibit A6 in the Appendix provides an opportunity to see how the uncertainty of each input affects the values for that input in the simulation. The $95 \%$ confidence intervals for the targeted WLs from Exhibit A4 are summarized in Table 2-2. Thus, with all of the probable errors activated for the best case scenario, the $95 \%$ confidence interval for the $32.4 \%$ targeted waste loading for SME Batch 261 is given by $(31.3,33.4)$. 
WSRC-TR-2004-00508

Revision 0

Table 2-2 Simulation Results for SME Batch 261's WL Target of 32.4\% with Smaller Analytical Uncertainties

\begin{tabular}{||c|c|c|c|c||}
\hline $\begin{array}{c}\text { Active Error } \\
\text { Indicator }\end{array}$ & $\begin{array}{c}\text { Active Error Descriptor } \\
\text { (1000 runs each) }\end{array}$ & $\begin{array}{c}\mathbf{9 5 \%} \\
\text { LCL }\end{array}$ & $\begin{array}{c}\mathbf{9 5 \%} \\
\text { UCL }\end{array}$ & $\begin{array}{c}\text { WL } \\
\text { Uncertainty }\end{array}$ \\
\hline 0 & All (0) & 31.3 & 33.4 & 1.05 \\
\hline 1 & Heel calcine solids (1) & 32.4 & 32.4 & 0.00 \\
\hline 2 & Heel SpG (2) & 32.4 & 32.4 & 0.00 \\
\hline 3 & Heel Chem Comps (3) & 31.7 & 33.0 & 0.65 \\
\hline 4 & Heel Volume (4) & 32.4 & 32.4 & 0.00 \\
\hline 5 & SRAT wt\% solids (5) & 32.1 & 32.7 & 0.30 \\
\hline 6 & SRAT SpG (6) & 32.3 & 32.5 & 0.10 \\
\hline 7 & SRAT Chem Comps (7) & 32.2 & 32.6 & 0.20 \\
\hline 8 & SRAT Volume (8) & 32.2 & 32.6 & 0.20 \\
\hline 9 & Frit Chem Comps (9) & 31.7 & 33.1 & 0.70 \\
\hline 10 & Frit lbs (10) & 32.2 & 32.6 & 0.20 \\
\hline 11 & Frit lbs from Can Decon (11) & 32.3 & 32.4 & 0.05 \\
\hline \multicolumn{4}{|l}{}
\end{tabular}

\subsection{ESTIMATING THE WL ATTAINED FOR A SME BATCH}

Table A3 in the Appendix provides information on estimated WLs for SME batches 234 through 265. This information is part of the SME acceptability spreadsheets, and the data presented represent the 4 samples for each of the SME batches. Included in this table are the batch number, the elemental lithium (Li) content for the sample (as a weight percent, wt\%), sum of oxides ( $w t \%$ ) for the sample, the $\mathrm{Li}_{2} \mathrm{O}$ content (wt \%) for the sample, and the $\mathrm{Li}_{2} \mathrm{O}$ content of the frit for the frit lot(s) used for that SME batch. This information is used to estimate the WL for each of the four samples for each SME batch through the formula given by Equation (1):

$$
\mathrm{WL}=100 \cdot\left(1-\frac{\mathrm{Li}_{2} \mathrm{O} /(\text { Sum of Oxides })}{\text { Frit }_{\mathrm{Li}_{2} \mathrm{O}} / 100}\right)
$$

The average of the 4 WLs is used as the estimated WL for the SME batch. If the four WLs are assumed to be a random sample of the possible WLs that might have been determined for the SME batch, they may be used to construct a 95\% confidence interval for the true WL (under the additional assumption of normality for these random variables). Figure 2-1 provides a plot of these $95 \%$ confidence intervals covering SME batches 233 through 265. The plotted " $X$ " represents the estimated WL with the vertical bar around this symbol representing the confidence interval for the WL. The length of the confidence intervals for the various batches differs due to the differences in the scatter (i.e., the standard deviation) of the 4 sample results for a given SME batch. 
WSRC-TR-2004-00508

Revision 0

Figure 2-1 Estimated WLs by SME Batch with 95\% Confidence Intervals Based upon 4 Samples

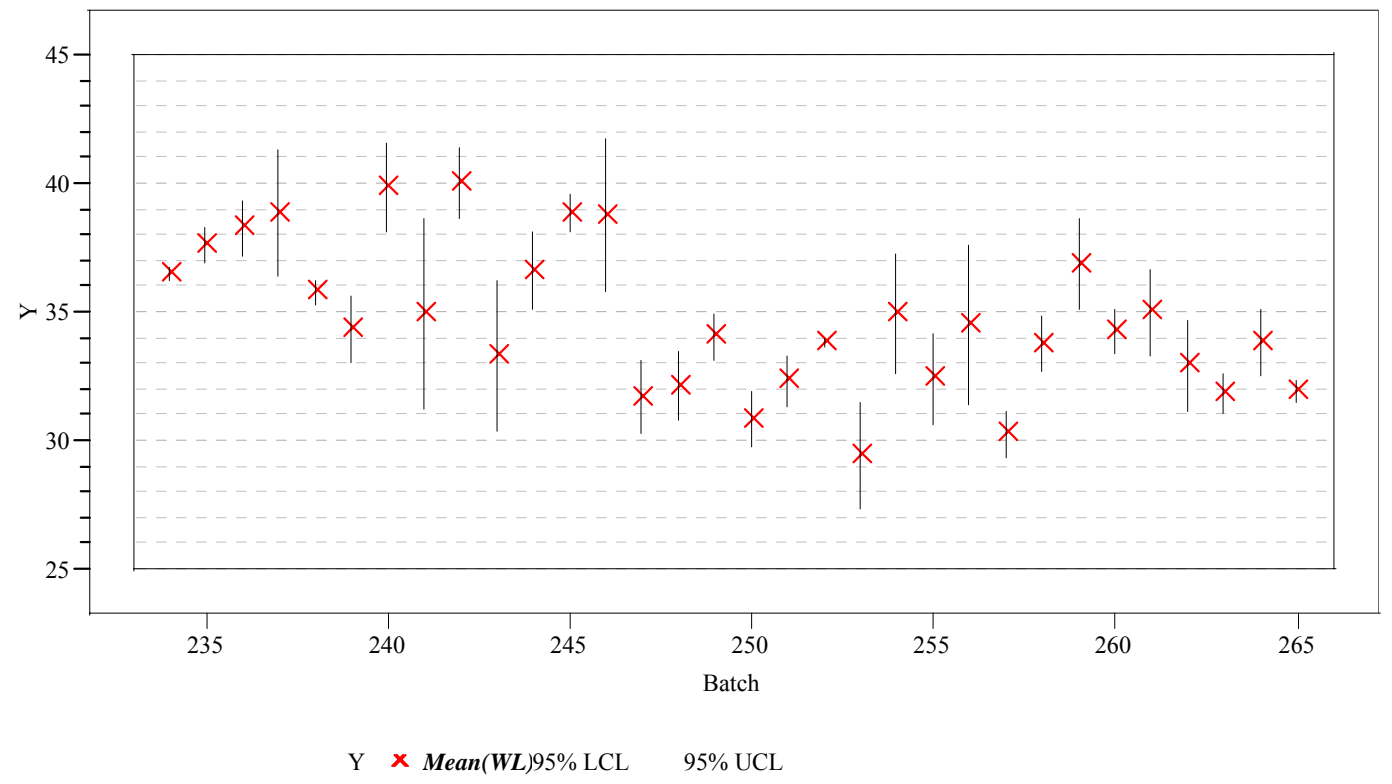

In Figure 2-2 the individual standard deviations have been pooled, and the pooled standard deviation used in determining the $95 \%$ confidence interval for the WL of each SME batch. In this case, the lengths of the confidence intervals are all the same, and the interval is given by WL \pm 1.1 with 1.1 representing the uncertainty of the estimated WL. Thus, using this approach for SME Batch 261, the estimated WL would be $35 \pm 1.1$ or $(33.9,36.1)$ with $95 \%$ confidence.

Figure 2-2 Estimated WLs by SME Batch with 95\% Confidence Intervals Based upon 4 Samples and a Pooled Estimate of the Standard Deviation

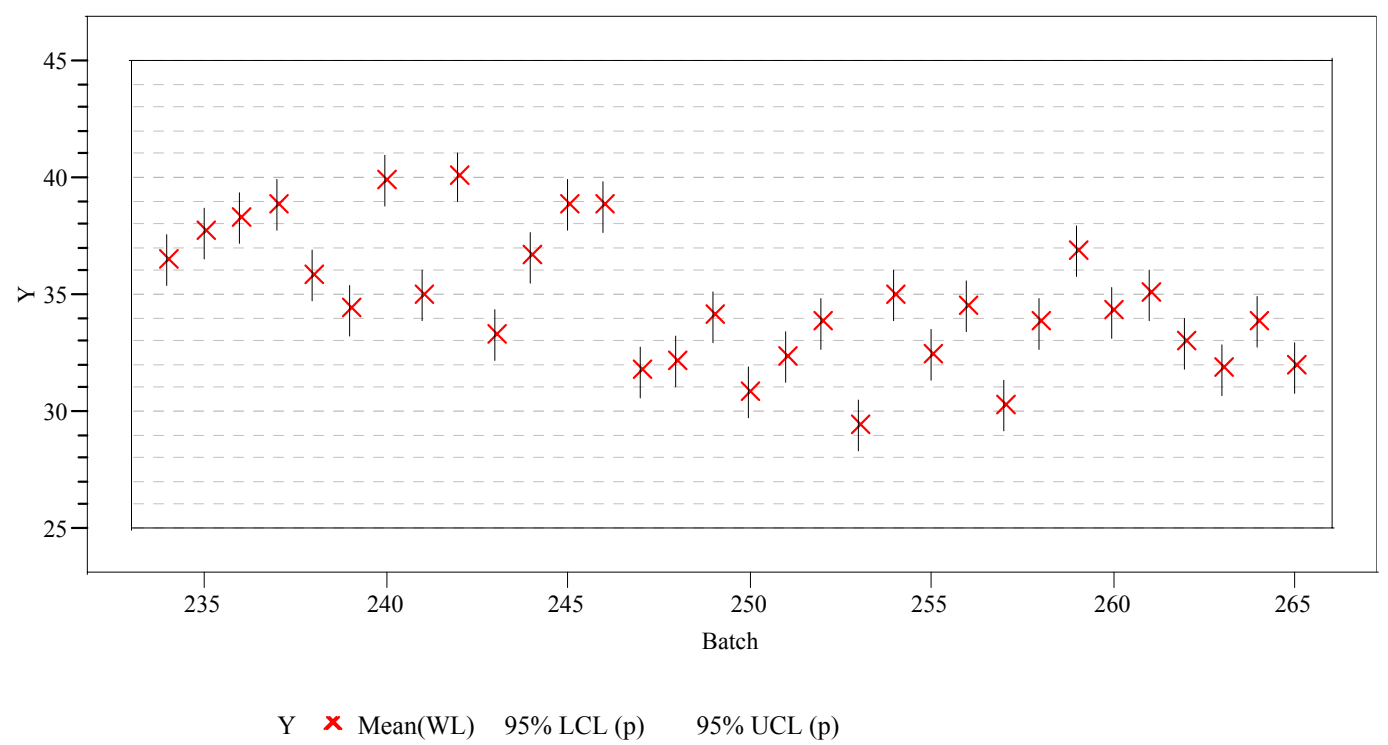




\subsection{UNCERTAINTIES IN ESTIMATING THE WL ATTAINED FOR A SME BATCH}

The uncertainty of the estimated WL also can be approached in a manner similar to that used for the targeted WL. The inputs to Equation (1) provide the starting place; their nominal values and estimated random uncertainties for SME Batch 261 are provided in Table A4 in the Appendix. The last row of this table provides the nominal $\mathrm{Li}_{2} \mathrm{O}$ content in the frit $\operatorname{lot}(\mathrm{s})$ used for this batch. The percent relative standard deviation (\% RSD) for this value was estimated using the $\mathrm{Li}_{2} \mathrm{O}$ frit values from Table A3. The \%RSD's for the elemental measurements are the same as those in the first \%RSD column of Table A2.

Exhibit A7 and Exhibit A8 in Appendix A provide the results of this simulation. Exhibit A7 provides a summary plot of the impact on the uncertainty of the estimated WL based upon the contribution of the different error categories. Exhibit A8 provides histograms and descriptive statistics for these results. Exhibit A9 in the Appendix provides a look at the impact of the input uncertainties on the values of the inputs to this determination. Table $2-3$ provides $95 \%$ confidence intervals for the estimated WL based upon the information of Exhibit A8. For SME Batch 261, the 95\% confidence interval for the estimated $\mathrm{WL}$ is $35.0 \pm 1.5$ or $(33.5,36.5)$ based on the random uncertainties of the inputs.

Table 2-3 Simulation Results for Uncertainties in Estimated WL for SME Batch 261

\begin{tabular}{||c|c|c|c|c|}
\hline \hline $\begin{array}{c}\text { Active Error } \\
\text { Indicator }\end{array}$ & $\begin{array}{c}\text { Active Error Descriptor } \\
(\mathbf{1 0 0 0} \text { runs each) }\end{array}$ & $\begin{array}{c}\mathbf{9 5 \%} \\
\text { LCL }\end{array}$ & $\begin{array}{c}\mathbf{9 5 \%} \\
\text { UCL }\end{array}$ & $\begin{array}{c}\text { WL } \\
\text { Uncertainty }\end{array}$ \\
\hline 0 & All (0) & 33.5 & 36.5 & 1.50 \\
\hline 1 & Aluminum (1) & 34.9 & 35.1 & 0.10 \\
\hline 2 & Boron (2) & 34.9 & 35.1 & 0.10 \\
\hline 3 & Calcium (3) & 34.9 & 35.1 & 0.10 \\
\hline 4 & Chromium (4) & 35.0 & 35.0 & 0.00 \\
\hline 5 & Copper (5) & 35.0 & 35.0 & 0.00 \\
\hline 6 & Iron (6) & 34.7 & 35.3 & 0.30 \\
\hline 7 & Potassium (7) & 35.0 & 35.0 & 0.00 \\
\hline 8 & Lithium (8) & 33.9 & 36.1 & 1.10 \\
\hline 9 & Magnesium (9) & 35.0 & 35.0 & 0.00 \\
\hline 10 & Manganese (10) & 35.0 & 35.0 & 0.00 \\
\hline 11 & Sodium (11) & 34.5 & 35.4 & 0.45 \\
\hline 12 & Nickel (12) & 34.9 & 35.1 & 0.10 \\
\hline 13 & Silicon (13) & 34.4 & 35.6 & 0.60 \\
\hline 14 & Titanium (14) & 35.0 & 35.0 & 0.00 \\
\hline 15 & Uranium (15) & 34.7 & 35.3 & 0.30 \\
\hline 16 & Zirconium (16) & 35.0 & 35.0 & 0.00 \\
\hline 17 & Frit 320 Li I $_{2} \mathrm{O}(17)$ & 34.7 & 35.3 & 0.30 \\
\hline
\end{tabular}

\subsection{CONTRASTING TARGETED AND ESTIMATED WASTE LOADINGS}

The results of the investigation suggest that the targeted WL for SME Batch 261 was in the interval (30.5, 34.1 ) with $95 \%$ confidence (this is the worst case scenario for analytical uncertainties) while the estimated WL for this batch was in the interval $(33.5,36.5)$ with the same confidence. Thus, these two intervals overlap somewhat at the low end of the estimated value and the high end of the targeted value. For the best case scenario, where the confidence interval for the targeted WL was $(31.3,33.4)$, this interval does not overlap with the interval for the estimated WL. 
How do the targeted and estimated waste loadings compare for other recent DWPF batches? Figure 2-3 provides a plot of the targeted and estimated WLs for batches 234 through 265. For most of the batches considered the estimated WLs are larger than the targeted WLs. There is some overlap of the confidence bands for the pairs of values but not as much as might be expected. One aspect common to both targeting and estimating WL is the normalization of the SME composition, whether the composition is targeted as part of the SME blending process or measured as part of the assessment of a SME batch. For SME blending, the normalization is inherent in the determination of the $\mathrm{wt} \% \mathrm{Li}_{2} \mathrm{O}$ as the ratio of the pounds of $\mathrm{Li}_{2} \mathrm{O}$ to the total pounds of the 16 oxides that currently are being monitored (see Table 2-3 for a listing of the corresponding cations). For the SME assessment, the normalization is explicitly handled in the calculation of WL for each sample by dividing the measured $\mathrm{Li}_{2} \mathrm{O}$ by the corresponding sum of oxides.

Figure 2-3 Plot of Targeted and Estimated Waste Loadings by Batch Number with Uncertainty Bands from Sensitivity Study

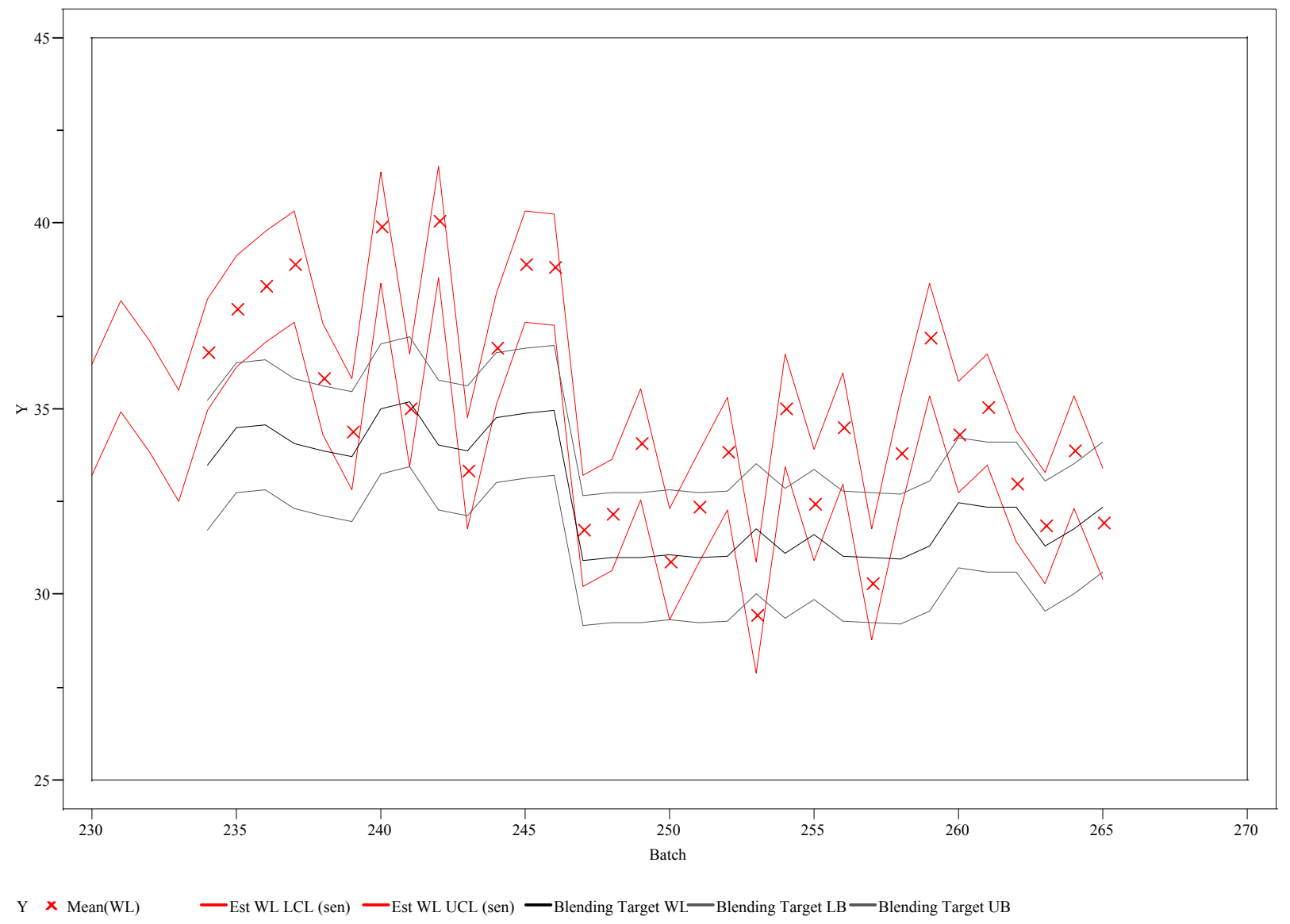

The normalization step in these processes, whether explicit or implicit, warrants a closer look. For the SME assessment (i.e., the estimation of WL), the normalization of the $\mathrm{Li}_{2} \mathrm{O}$ measurement for a sample by dividing by the corresponding sum of oxides suggests that a low sum of oxides for a sample may imply a lower $\mathrm{Li}_{2} \mathrm{O}$ measurement for that sample. Exhibit A10 in the Appendix provides a series of plots and linear regressions of $\mathrm{Li}_{2} \mathrm{O}$ versus sum of oxides for samples from SME batches 227 through 265. The results for these 39 batches may be summarized as follows: only 6 of the 39 batches revealed a statistically significant (at $\sim 5 \%$ significance level) correlation between the $\mathrm{Li}_{2} \mathrm{O}$ content and total sum of oxides for a sample. There is little indication from these data that a low sum of oxides for a sample indicates a low $\mathrm{Li}_{2} \mathrm{O}$ recovery for the sample. 
As stated above, the targeted WL is driven by the planned $\mathrm{Li}_{2} \mathrm{O}$ concentration for the batch that is determined by the ratio of the pounds of $\mathrm{Li}_{2} \mathrm{O}$ to the total pounds of the 16 oxides that are monitoring for the blending process. Can minor oxides that are not accounted for during this normalization affect the targeted WL? Table 2-4 attempts to provide insight into the answer for this question by revisiting the targeted WL for each batch from 233 through 265. For each of these batches, four values of WL are provided: the original targeted WL and three WL values adjusted to reflect 1,2 , and $3 \mathrm{wt} \%$ for minor oxides in the final glass product. These minor oxides are not part of the 16 currently being measured and, therefore, do not contribute to the sum of oxides. Thus, even if the measured values for the 16 oxides were unbiased relative to their true values, the sum of oxides would always be less than $100 \%$; that is, the sum of the measurements for these 16 oxides would recover less than $100 \%$.

Table 2-4 Impact of the Sum of the Concentrations of the 16 Oxides Being Monitored on Targeted WL

\begin{tabular}{|c|c|c|c|c|}
\hline Batch & $\begin{array}{c}\text { Blending } \\
\text { Targeted } \\
\text { WL }\end{array}$ & $\begin{array}{c}\text { Targeted WL } \\
\text { Assuming 99\% } \\
\text { Recovery }\end{array}$ & $\begin{array}{c}\text { Targeted WL } \\
\text { Assuming 98\% } \\
\text { Recovery }\end{array}$ & $\begin{array}{c}\text { Targeted WL } \\
\text { Assuming 97\% } \\
\text { Recovery }\end{array}$ \\
\hline 234 & 33.49 & 34.15 & 34.82 & 35.48 \\
\hline 235 & 34.52 & 35.17 & 35.83 & 36.48 \\
\hline 236 & 34.60 & 35.25 & 35.91 & 36.56 \\
\hline 237 & 34.09 & 34.75 & 35.40 & 36.06 \\
\hline 238 & 33.90 & 34.56 & 35.23 & 35.89 \\
\hline 239 & 33.75 & 34.41 & 35.07 & 35.74 \\
\hline 240 & 35.03 & 35.68 & 36.33 & 36.98 \\
\hline 241 & 35.22 & 35.87 & 36.51 & 37.16 \\
\hline 242 & 34.04 & 34.70 & 35.36 & 36.02 \\
\hline 243 & 33.90 & 34.56 & 35.23 & 35.89 \\
\hline 244 & 34.77 & 35.43 & 36.08 & 36.73 \\
\hline 245 & 34.92 & 35.57 & 36.22 & 36.87 \\
\hline 246 & 34.99 & 35.64 & 36.29 & 36.94 \\
\hline 247 & 30.94 & 31.63 & 32.32 & 33.01 \\
\hline 248 & 31.00 & 31.69 & 32.38 & 33.07 \\
\hline 249 & 31.02 & 31.71 & 32.40 & 33.09 \\
\hline 250 & 31.10 & 31.79 & 32.48 & 33.17 \\
\hline 251 & 31.00 & 31.69 & 32.38 & 33.07 \\
\hline 252 & 31.07 & 31.75 & 32.44 & 33.13 \\
\hline 253 & 31.80 & 32.48 & 33.16 & 33.84 \\
\hline 254 & 31.12 & 31.81 & 32.50 & 33.19 \\
\hline 255 & 31.62 & 32.31 & 32.99 & 33.67 \\
\hline 256 & 31.05 & 31.74 & 32.43 & 33.12 \\
\hline 257 & 31.01 & 31.70 & 32.39 & 33.08 \\
\hline 258 & 30.97 & 31.66 & 32.35 & 33.04 \\
\hline 259 & 31.32 & 32.01 & 32.70 & 33.38 \\
\hline 260 & 32.48 & 33.15 & 33.83 & 34.50 \\
\hline 261 & 32.39 & 33.06 & 33.74 & 34.41 \\
\hline 262 & 32.39 & 33.07 & 33.74 & 34.42 \\
\hline 263 & 31.31 & 31.99 & 32.68 & 33.37 \\
\hline 264 & 31.77 & 32.46 & 33.14 & 33.82 \\
\hline 265 & 32.38 & 33.05 & 33.73 & 34.41 \\
\hline
\end{tabular}

Note that as the concentration of the group of minor oxides in the glass product increases from 1 to $3 \mathrm{wt} \%$ (i.e., the recovery decreases from 99 to $97 \%$ ) there is a dramatic effect on the targeted WL. Even if the 
minors are at only $2 \mathrm{wt} \%$, the resulting adjustment increases $\mathrm{WL}$ by over a percentage point. For example, the targeted WL for SME Batch 261 goes from $32.38 \%$ to $33.64 \%$.

The presence of minor oxides also affects the estimation of WL determined for each of the SME samples. Since the sum of oxides for each sample is used to normalize the $\mathrm{Li}_{2} \mathrm{O}$ content of the sample, if the sum of oxides for the 16 monitored oxides is inherently low, the $\mathrm{Li}_{2} \mathrm{O}$ content is being inflated and the estimated WL is understated. Table $2-5$ explores the effects of less than $100 \%$ recovery of the oxides by monitoring only the set of 16 routinely measured oxides. Once again, the minors are assumed to represent 1, 2, and 3 $\mathrm{wt} \%$ of the final glass product, and the normalization of the $\mathrm{Li}_{2} \mathrm{O}$ content of each sample is adjusted appropriately.

Table 2-5 Impact of the Sum of the 16 Oxides Being Monitored on Estimated WL

\begin{tabular}{|c|c|c|c|c|}
\hline Batch & $\begin{array}{c}\text { Estimated } \\
\text { WL for Batch }\end{array}$ & $\begin{array}{c}\text { Estimated WL Assuming } \\
99 \% \text { Recovery }\end{array}$ & $\begin{array}{c}\text { Estimated WL Assuming } \\
98 \% \text { Recovery }\end{array}$ & $\begin{array}{c}\text { Estimated WL Assuming } \\
97 \% \text { Recovery }\end{array}$ \\
\hline 227 & 32.38 & 33.06 & 33.74 & 34.41 \\
\hline 228 & 31.95 & 32.63 & 33.31 & 33.99 \\
\hline 229 & 31.42 & 32.11 & 32.79 & 33.48 \\
\hline 230 & 34.74 & 35.39 & 36.04 & 36.69 \\
\hline 231 & 36.42 & 37.06 & 37.70 & 38.33 \\
\hline 232 & 35.37 & 36.01 & 36.66 & 37.30 \\
\hline 233 & 34.02 & 34.68 & 35.34 & 36.00 \\
\hline 234 & 36.48 & 37.12 & 37.76 & 38.39 \\
\hline 235 & 37.65 & 38.28 & 38.90 & 39.52 \\
\hline 236 & 38.30 & 38.92 & 39.53 & 40.15 \\
\hline 237 & 38.86 & 39.47 & 40.09 & 40.70 \\
\hline 238 & 35.81 & 36.45 & 37.10 & 37.74 \\
\hline 239 & 34.35 & 35.01 & 35.67 & 36.32 \\
\hline 240 & 39.89 & 40.49 & 41.09 & 41.69 \\
\hline 241 & 34.98 & 35.63 & 36.28 & 36.93 \\
\hline 242 & 40.04 & 40.64 & 41.24 & 41.84 \\
\hline 243 & 33.30 & 33.97 & 34.63 & 35.30 \\
\hline 244 & 36.62 & 37.25 & 37.89 & 38.52 \\
\hline 245 & 38.87 & 39.48 & 40.09 & 40.70 \\
\hline 246 & 38.79 & 39.40 & 40.01 & 40.63 \\
\hline 247 & 31.72 & 32.40 & 33.08 & 33.76 \\
\hline 248 & 32.15 & 32.82 & 33.50 & 34.18 \\
\hline 249 & 34.06 & 34.72 & 35.38 & 36.04 \\
\hline 250 & 30.84 & 31.53 & 32.23 & 32.92 \\
\hline 251 & 32.35 & 33.02 & 33.70 & 34.38 \\
\hline 252 & 33.81 & 34.47 & 35.14 & 35.80 \\
\hline 253 & 29.41 & 30.11 & 30.82 & 31.53 \\
\hline 254 & 34.98 & 35.63 & 36.28 & 36.93 \\
\hline 255 & 32.42 & 33.10 & 33.77 & 34.45 \\
\hline 256 & 34.49 & 35.15 & 35.80 & 36.46 \\
\hline 257 & 30.28 & 30.97 & 31.67 & 32.37 \\
\hline 258 & 33.79 & 34.45 & 35.12 & 35.78 \\
\hline 259 & 36.89 & 37.52 & 38.15 & 38.78 \\
\hline 260 & 34.28 & 34.93 & 35.59 & 36.25 \\
\hline 261 & 35.00 & 35.65 & 36.30 & 36.95 \\
\hline 262 & 32.94 & 33.61 & 34.28 & 34.95 \\
\hline 263 & 31.82 & 32.51 & 33.19 & 33.87 \\
\hline 264 & 33.85 & 34.51 & 35.17 & 35.84 \\
\hline 265 & 31.92 & 32.60 & 33.28 & 33.96 \\
\hline
\end{tabular}


Based upon the earlier results, the normalization of the $\mathrm{Li}_{2} \mathrm{O}$ in a SME sample by the sum of oxides for the sample may not be necessary; thus, the role of minor oxides may have a potentially bigger impact on the targeted WL. To investigate this possibility in more detail, one additional comparison is provided in the form of Figure 2-4. In this figure, the targeted WLs adjusted for $98 \%$ recovery are compared to estimate WLs by batch with the calculation of the estimated WLs not involving a normalization step. Uncertainty limits (at 95\% confidence) are added to the plot for the targeted WLs based upon the \pm 1.75 value in Table 2-1. Uncertainty limits (at 95\% confidence) are added to the plot for the estimated WL based upon the \pm 1.50 value in Table $2-3$. This is a conservative bound on these errors since errors in the sum of oxides do not come into play without the normalization step. Although some improvement is seen in the comparisons of the targeted versus estimated WLs for these batches, there are still some batches for which the two uncertainty bands do not overlap.

Figure 2-4 Plot of Targeted and Estimated Waste Loadings Adjusted for $\mathbf{9 8 \%}$ Recovery by Batch Number

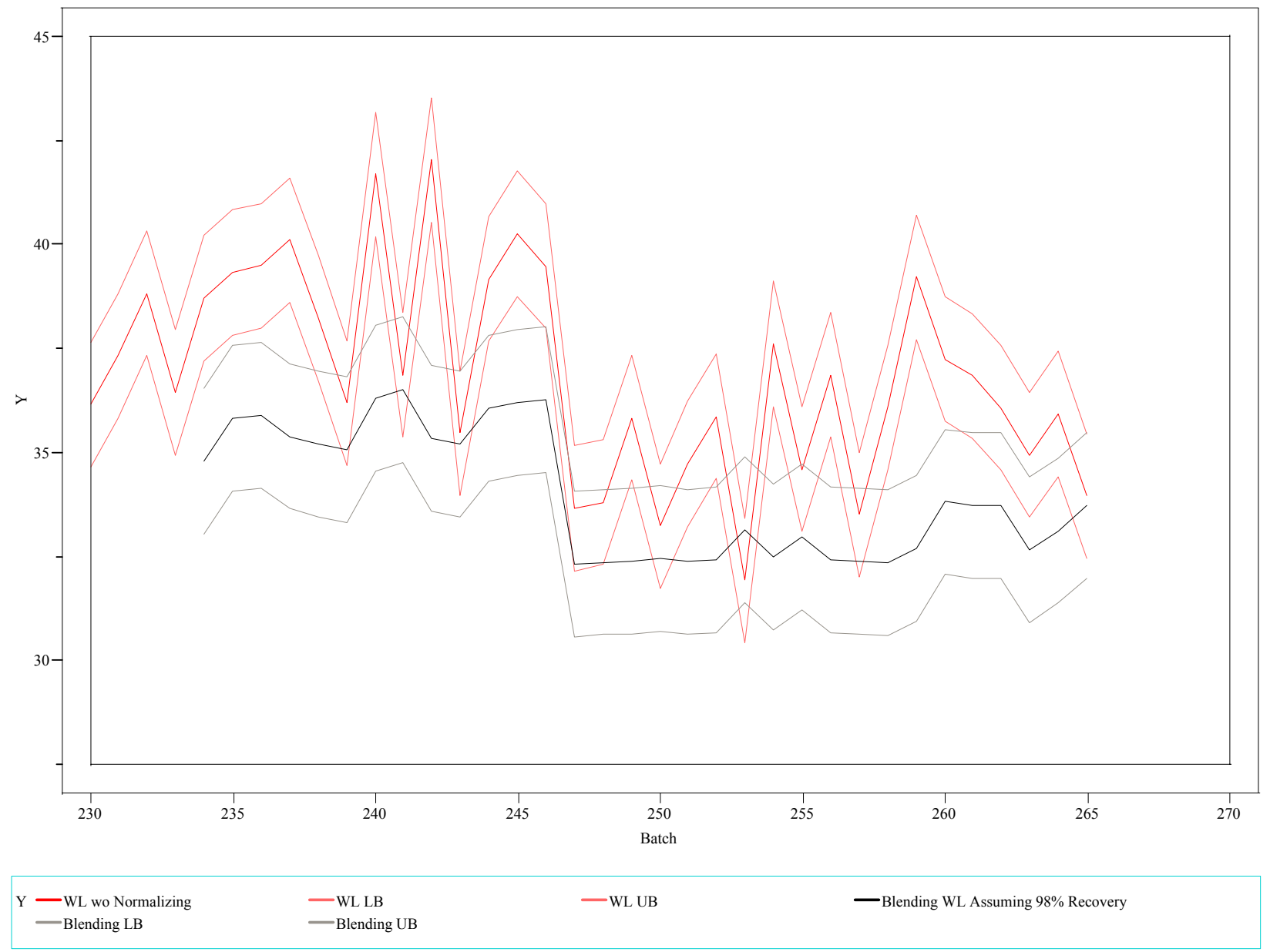




\subsection{IMPACT OF A POTENTIAL BIAS ON WL'S}

In the analyses conducted above, the sensitivity of estimated WLs to random variations in the inputs to the estimation process was investigated. This section considers the impact of a potential bias in one of the inputs, namely the $\mathrm{Li}_{2} \mathrm{O}$ content in the SME samples. The discussion presented here is motivated by a result presented in [5], which indicates that in prototypical testing the DWPF sampling mechanism yielded samples slightly $(\sim 1.5 \%)$ low in $\mathrm{Li}_{2} \mathrm{O}$ content. The impact of such a bias on the reporting of the DWPF glass product was investigated and found to be acceptable as documented in DWPF's Wasteform Qualification Report [6]. The question to be addressed in this section is how big of an impact could a $1.5 \%$ bias in the $\mathrm{Li}_{2} \mathrm{O}$ measurement have on the estimated WL? To answer the question, the information presented in Table A. 3 for SME batches 234 through 265 was revisited. Assuming that the $\mathrm{Li}_{2} \mathrm{O}$ measurements of the SME samples are biased low by $1.5 \%$, an adjustment was made to each of these measured values: the value was multiplied by 1.015 . WLs were then estimated using the adjusted $\mathrm{Li}_{2} \mathrm{O}$ values, both normalized and not normalized, and the resulting values are presented in Table 2-6. To facilitate the comparisons, WLs computed using the unadjusted, not-normalized $\mathrm{Li}_{2} \mathrm{O}$ values are also presented in this table.

Table 2-6 Impact of a 1.5\% $\mathrm{Li}_{2} \mathrm{O}$ Bias on Estimated WL

\begin{tabular}{|c|c|c|c|c|}
\hline Batch & $\begin{array}{c}\text { Estimated WL for } \\
\text { Batch }\end{array}$ & $\begin{array}{l}\text { Estimated WL Without } \\
\text { Normalizing } \mathrm{Li}_{2} \mathrm{O}\end{array}$ & $\begin{array}{c}\text { Estimated WL After } \\
\text { Adjusting for a } 1.5 \% \\
\mathrm{Li}_{2} \mathrm{O} \text { Bias and } \\
\text { Normalizing }\end{array}$ & $\begin{array}{c}\text { Estimated WL After } \\
\text { Adjusting for a } 1.5 \% \\
\mathrm{Li}_{2} \mathrm{O} \text { Bias Without } \\
\text { Normalizing }\end{array}$ \\
\hline 234 & 36.48 & 38.72 & 35.53 & 37.81 \\
\hline 235 & 37.65 & 39.35 & 36.72 & 38.44 \\
\hline 236 & 38.30 & 39.50 & 37.37 & 38.59 \\
\hline 237 & 38.86 & 40.12 & 37.95 & 39.23 \\
\hline 238 & 35.81 & 38.24 & 34.85 & 37.32 \\
\hline 239 & 34.35 & 36.21 & 33.37 & 35.25 \\
\hline 240 & 39.89 & 41.71 & 38.99 & 40.83 \\
\hline 241 & 34.98 & 36.88 & 34.00 & 35.94 \\
\hline 242 & 40.04 & 42.05 & 39.15 & 41.18 \\
\hline 243 & 33.30 & 35.48 & 32.30 & 34.51 \\
\hline 244 & 36.62 & 39.18 & 35.67 & 38.27 \\
\hline 245 & 38.87 & 40.28 & 37.95 & 39.38 \\
\hline 246 & 38.79 & 39.49 & 37.87 & 38.58 \\
\hline 247 & 31.72 & 33.67 & 30.69 & 32.68 \\
\hline 248 & 32.15 & 33.82 & 31.13 & 32.83 \\
\hline 249 & 34.06 & 35.85 & 33.07 & 34.88 \\
\hline 250 & 30.84 & 33.25 & 29.80 & 32.25 \\
\hline 251 & 32.35 & 34.74 & 31.33 & 33.76 \\
\hline 252 & 33.81 & 35.89 & 32.82 & 34.93 \\
\hline 253 & 29.41 & 31.95 & 28.35 & 30.93 \\
\hline 254 & 34.98 & 37.63 & 34.01 & 36.69 \\
\hline 255 & 32.42 & 34.61 & 31.41 & 33.63 \\
\hline 256 & 34.49 & 36.88 & 33.51 & 35.93 \\
\hline 257 & 30.28 & 33.53 & 29.23 & 32.53 \\
\hline 258 & 33.79 & 36.10 & 32.80 & 35.15 \\
\hline 259 & 36.89 & 39.23 & 35.94 & 38.32 \\
\hline 260 & 34.28 & 37.26 & 33.29 & 36.32 \\
\hline 261 & 35.00 & 36.86 & 34.03 & 35.92 \\
\hline 262 & 32.94 & 36.10 & 31.94 & 35.14 \\
\hline 263 & 31.82 & 34.95 & 30.80 & 33.98 \\
\hline 264 & 33.85 & 35.94 & 32.86 & 34.98 \\
\hline 265 & 31.92 & 33.97 & 30.90 & 32.98 \\
\hline
\end{tabular}


The question of interest from the data in Table 2-6 is: How much of an impact does a potential 1.5\% bias in the $\mathrm{Li}_{2} \mathrm{O}$ sample measurements have on the resulting WL calculation. This question is addressed by Figure 2-5, which provides a direct comparison between the unadjusted and adjusted WL values (i.e., a comparison between columns 2 and 4 of Table 2-6).

Figure 2-5 A Linear Fit of the WLs Calculated Using the Adjusted $\mathrm{Li}_{2} \mathrm{O}$ Values Versus the Unadjusted WLs Linear Fit: WL adjusted for $1.5 \%$ bias $=-0.979+1 \mathrm{WL}$

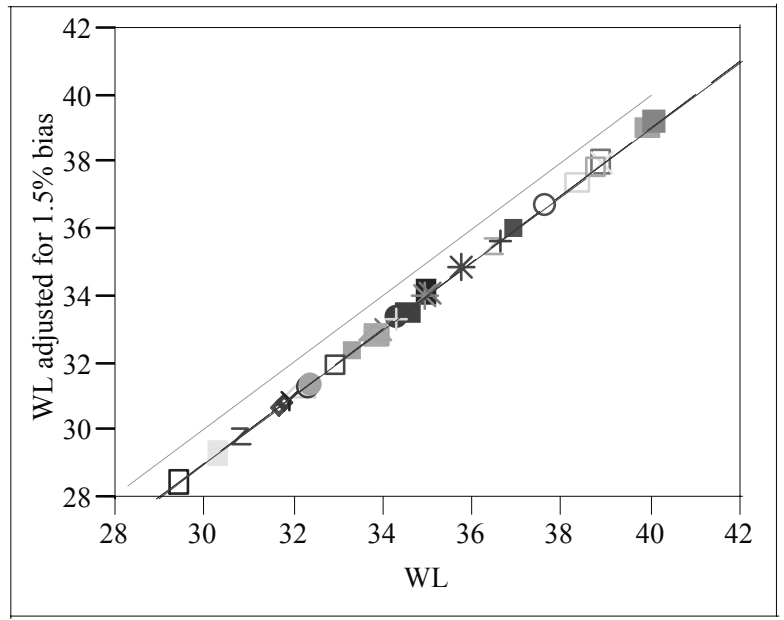

The dashed, diagonal line of Figure 2-5 represents situation where the adjusted and unadjusted WLs are equal. Since the plotted points and the fitted line are below the diagonal, the adjusted WLs are consistently less than their unadjusted counterparts. From the equation of the fitted model above the figure, the estimated WLs calculated using the bias-corrected $\mathrm{Li}_{2} \mathrm{O}$ values are on-average 0.98 smaller than those reported by DWPF. Thus, for a reported WL of 34\%, if the WL were to be calculated using a bias-corrected value for $\mathrm{Li}_{2} \mathrm{O}$, the resulting $\mathrm{WL}$ would be $\sim 33 \%$.

How about the impact of the potential $\mathrm{Li}_{2} \mathrm{O}$ bias in the SME sampling on WL targeting? Since the blending process accounts for the SME heel, any bias in $\mathrm{Li}_{2} \mathrm{O}$ content of the heel would have an effect on WL targeting. Since the heel accounts for $\sim 40 \%$ of the total mass (of the 16 elements being tracked) determined during the blending process, the effect of a $1.5 \%$ bias would not be as significant in WL targeting as it is in the estimating of the WL at the SME. For the case where the SME heel accounts for $\sim 40 \%$, the WL targeted by the blending process would be overstated by $\sim 0.39$ (i.e., a nominal target of $34 \%$, would actually be only $\sim 33.6 \%$ ).

Another question of interest is the impact of the potential bias in explaining the gap between the targeted versus estimated WLs. Figure 2-6 provides a plot of the fourth column of Table 2-4 (i.e., the Blend WL Assuming 98\% Recovery) and the fourth column of Table 2-6 (i.e., the Estimated WL After Correcting for a $1.5 \% \mathrm{Li}_{2} \mathrm{O}$ Bias and Normalizing) versus $\mathrm{SME}$ batch number. Even without the introduction of any uncertainties, these two plots are seen to overlap to a great extent. 
WSRC-TR-2004-00508

Revision 0

Figure 2-6 Targeted versus Estimated WLs for SME Batches 234 through 265

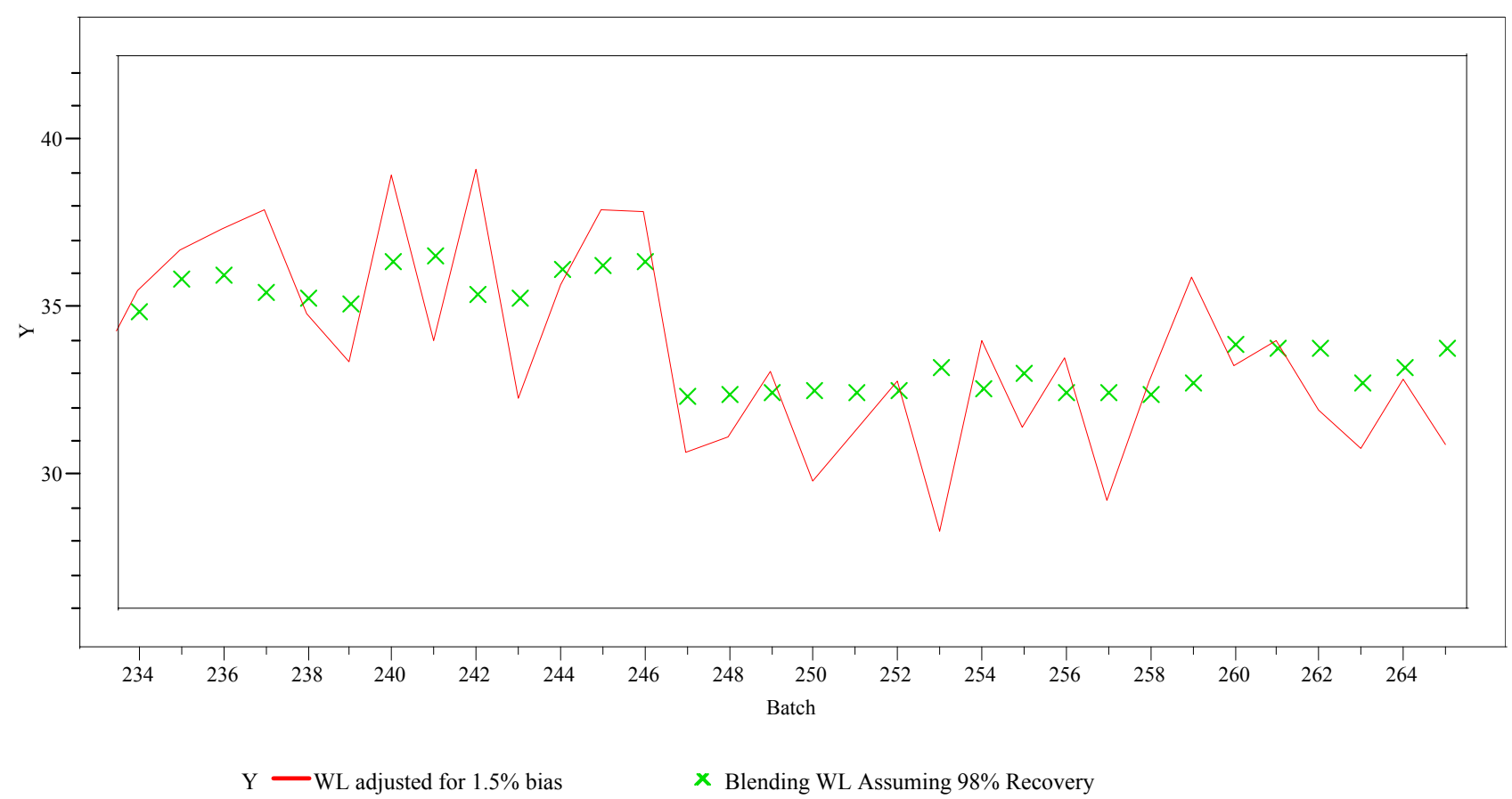

From the discussion above, it is obvious that even a small $\sim 1.5 \%$ bias in the $\mathrm{Li}_{2} \mathrm{O}$ measurements of SME samples can have a significant impact on the targeting and estimating of WLs. As seen in Figure 2-6, adjusting the targeted WL for a less than perfect recovery ( $98 \%$ for this situation) using the 16 oxides being tracked and adjusting the estimated WL for a $1.5 \%$ bias while also normalizing by the sum of oxides seem to bring these two processes more in line. Thus, the normalization of the $\mathrm{Li}_{2} \mathrm{O}$ content in each SME sample by the sample's sum of oxides (since this almost always leads to a larger $\mathrm{Li}_{2} \mathrm{O}$ value)

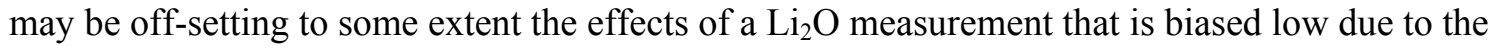
sampling mechanism. 


\subsection{CONCLUSIONS}

In this report, the calculations used to target WL during blending decisions and those used to estimate WL during SME processing are investigated to explore the sensitivities of these calculations to the random uncertainties of their inputs. For the calculations used to target WLs, the random uncertainties in the inputs lead to an uncertainty, at approximately $95 \%$ confidence, in the targeted WL of \pm 1.05 to \pm 1.75 points depending on how the errors in the inputs are represented. For the calculations used to estimate the WL for a given SME batch, the random uncertainties of the inputs to this calculation lead to an uncertainty, at approximately $95 \%$ confidence, in the estimated WL of \pm 1.50 points.

Since one would expect to see agreement between the WL calculations of the targeting process and the WL calculations of the estimating process, comparisons between these WLs for SME batches 234 through 265 were conducted. The comparisons suggested that the targeted WLs and estimated WLs for these batches did not track each other as closely as would be expected based upon their random variations as outlined in this report. In an effort to reconcile the targeted and estimated WLs some issues were identified:

- During the blending process, the $\mathrm{Li}_{2} \mathrm{O}$ content planned for the next SME batch is normalized using the sum of oxides for the 16 elements being tracked (i.e., the presence of minor oxides that might account for $\sim 1$ or $2 \%$ of the SME is not accounted for). This may lead to the targeted WL being understated.

- If there is a small $(\sim 1.5 \%)$ bias in the measured $\mathrm{Li}_{2} \mathrm{O}$ content of the SME samples due to the Hydragard $\AA /$ peanut vial sampling system (as seen in a prototypical test of this system conducted by Steimke in 1995), it could have an effect on the estimated WL (the WL value as a percentage could be overstated $\sim 1 \%$ ) and to a lesser extent on the targeted WL (the targeted WL value as a percentage could be overstated $\sim 0.4 \%$ ).

- The normalization of the $\mathrm{Li}_{2} \mathrm{O}$ content of each SME sample using the sample's sum of oxides, while not suggested by the data (i.e., there does not appear to be a correlation between a low lithium recovery and a low sum of oxides for the SME samples), may actually be lessening the impact of the potential bias in the $\mathrm{Li}_{2} \mathrm{O}$ measurements for the SME samples. 
WSRC-TR-2004-00508

Revision 0

This page intentionally left blank. 


\subsection{REFERENCES}

[1] Brown, K.G., R.L. Postles, T.B. Edwards, "SME Acceptability Determination for DWPF Process Control (U),”WSRC-TR-95-000364, Revision 4, August 30, 2002.

[2] Patel, P.M., "Technical Task Request: Statistical Analysis of DWPF Process Data and Lab Analytical Data (U)," HLW/DWPF/TTR-03-0020, Revision 0, December 9, 2003.

[3] SAS Institute, JMP ${ }^{\circledR}$ : Statistics and Graphics Guide, Version 5, SAS Institute, Inc., Cary, NC, 2002.

[4] Edwards, T.B., “A Statistical Review of Analytical Laboratory Measurements from DWPF's Macrobatch 1and Macrobatch 2 (U)," WSRC-TR-2003-00045, Revision 0, January 22, 2003.

[5] Steimke, J.L., "Results from Tests of TFL Hydragard Loop (U)," WSRC-TR-94-0598, March, 1995.

[6] Plodinec, M.J., S.L. Marra, T.B. Edwards, and C.J. Coleman, "Reporting the Chemical Composition of the DWPF Product (U)," WSRC-IM-91-116-2, Revision 1, December, 1995. 
WSRC-TR-2004-00508

Revision 0

This page intentionally left blank. 
WSRC-TR-2004-00508

Revision 0

APPENDIX: Tables and Exhibits 
WSRC-TR-2004-00508

Revision 0

This page intentionally left blank. 
WSRC-TR-2004-00508

Revision 0

Table A1. WL Targets for Batches 234 through 265

\begin{tabular}{|c|c|c|}
\hline $\begin{array}{c}\text { Batch } \\
\text { Number }\end{array}$ & $\begin{array}{c}\text { Targeted } \\
\text { WL }\end{array}$ & $\begin{array}{c}\mathrm{Li} 2 \mathrm{O}(\mathrm{wt} \%) \\
\text { in Frit } 320\end{array}$ \\
\hline 234 & 33.49 & 8.0450 \\
\hline 235 & 34.52 & 8.1025 \\
\hline 236 & 34.60 & 8.1025 \\
\hline 237 & 34.09 & 8.1090 \\
\hline 238 & 33.90 & 8.1090 \\
\hline 239 & 33.75 & 8.1090 \\
\hline 240 & 35.03 & 8.1090 \\
\hline 241 & 35.22 & 8.1090 \\
\hline 242 & 34.04 & 8.1090 \\
\hline 243 & 33.90 & 8.1090 \\
\hline 244 & 34.77 & 8.1090 \\
\hline 245 & 34.92 & 8.1136 \\
\hline 246 & 34.99 & 8.1136 \\
\hline 247 & 30.94 & 8.1136 \\
\hline 248 & 31.00 & 8.1136 \\
\hline 249 & 31.02 & 8.1136 \\
\hline 250 & 31.10 & 8.1163 \\
\hline 251 & 31.00 & 8.1163 \\
\hline 252 & 31.07 & 8.1163 \\
\hline 253 & 31.80 & 8.1163 \\
\hline 254 & 31.12 & 8.1163 \\
\hline 255 & 31.62 & 8.1163 \\
\hline 256 & 31.05 & 8.1163 \\
\hline 257 & 31.01 & 8.1163 \\
\hline 258 & 30.97 & 8.1163 \\
\hline 259 & 31.32 & 8.1163 \\
\hline 260 & 32.48 & 8.1163 \\
\hline 261 & 32.39 & 8.1163 \\
\hline 262 & 32.39 & 8.1163 \\
\hline 263 & 31.31 & 8.1163 \\
\hline 264 & 31.77 & 8.1163 \\
\hline 265 & 32.10 & 8.1163 \\
\hline
\end{tabular}


WSRC-TR-2004-00508

Revision 0

Table A2. Inputs and Their Uncertainties for Targeting WL

\begin{tabular}{|c|c|c|c|}
\hline & Nominal Value & Norminal +/- Normal Error with & Norminal +/- Normal Error with \\
\hline & in Blending & \% Relative Standard Deviation & \% Relative Standard Deviation \\
\hline Input & for SME Batch 261 & Assuming n=1 Sample & Assuming Nominal \# of Samples \\
\hline (1) SME Heel Calcined wt $\%$ solids wt $\%$ (s) rand & 41.253333 & 1.6 & 0.800 \\
\hline (2) SME Heel Specific gravity spgr rand & 1.41575 & 1.1 & 0.550 \\
\hline (3) SME Heel Aluminum wt\% (v) rand & 2.55575 & 4.1 & 2.050 \\
\hline (3) SME Heel Boron wt $\%(v)$ rand & 1.5445 & 3.5 & 1.750 \\
\hline (3) SME Heel Calcium wt\% (v) rand & 0.89225 & 6 & 3.000 \\
\hline (3) SME Heel Chromium wt\% (v) rand & 0.074 & 17.1 & 8.550 \\
\hline (3) SME Heel Copper wt $\%$ (v) rand & 0.02425 & 15.2 & 7.600 \\
\hline (3) SME Heel Iron wt $\%$ (v) rand & 9.413 & 3.3 & 1.650 \\
\hline (3) SME Heel Potassium wt $\%$ (v) rand & 0.16325 & 20 & 10.000 \\
\hline (3) SME Heel Lithium wt\% (v) rand & 2.35175 & 2.1 & 1.050 \\
\hline (3) SME Heel Magnesium wt\% (v) rand & 0.676 & 3.5 & 1.750 \\
\hline (3) SME Heel Manganese wt\% (v) rand & 1.131 & 3.8 & 1.900 \\
\hline (3) SME Heel Sodium wt $\%$ (v) rand & 8.046 & 6 & 3.000 \\
\hline (3) SME Heel Nickel wt\% (v) rand & 0.468 & 17.7 & 8.850 \\
\hline (3) SME Heel Silicon wt $\%$ (v) rand & 22.203625 & 2.1 & 1.050 \\
\hline (3) SME Heel Titanium wt $\%(v)$ rand & 0.0365 & 25 & 12.500 \\
\hline (3) SME Heel Uranium wt\% (v) rand & 3.29175 & 9.5 & 4.750 \\
\hline (3) SME Heel Zirconium wt\% (v) rand & 0.068 & 15.6 & 7.800 \\
\hline (4) SME Heel Volume (gals) rand & 2915 & 1 & 1 \\
\hline (5) SRAT Total wt $\%$ solids wt $\%$ (s) rand & 24.1305746 & 2.9 & 1.184 \\
\hline (6) SRAT Specific gravity spgr rand & 1.18191447 & 1.1 & 0.449 \\
\hline (7) SRAT Aluminum wt $\%$ (s) rand & 5.1704 & 3.6 & 1.470 \\
\hline (7) SRAT Boron $\mathrm{wt} \%(\mathrm{~s})$ rand & 0.0308 & 25 & 10.206 \\
\hline (7) SRAT Calcium wt $\%$ (s) rand & 2.1828 & 5.7 & 2.327 \\
\hline (7) SRAT Chromium wt\% (s) rand & 0.1408 & 25 & 10.206 \\
\hline (7) SRAT Copper wt\% (s) rand & 0.0456 & 25 & 10.206 \\
\hline (7) SRAT Iron $w t \%(s)$ rand & 20.941 & 3.6 & 1.470 \\
\hline (7) SRAT Potassium $\mathrm{wt} \%$ (s) rand & 0.0634 & 25 & 10.206 \\
\hline (7) SRAT Lithium $w t \%(s)$ rand & 0.063 & 25 & 10.206 \\
\hline (7) SRAT Magnesium wt\% (s) rand & 1.6738 & 3.4 & 1.388 \\
\hline (7) SRAT Manganese $w t \%(s)$ rand & 2.7936 & 3.3 & 1.347 \\
\hline (7) SRAT Sodium $w t \%(s)$ rand & 6.431 & 3.4 & 1.388 \\
\hline (7) SRAT Nickel wt\% (s) rand & 1.0484 & 11.6 & 4.736 \\
\hline (7) SRAT Silicon $w t \%(s)$ rand & 0.96 & 22 & 8.981 \\
\hline (7) SRAT Titanium $w t \%(s)$ rand & 0.0152 & 8.1 & 3.307 \\
\hline (7) SRAT Uranium wt $\%$ (s) rand & 6.8894 & 5.3 & 2.164 \\
\hline (7) SRAT Zirconium wt $\%$ (s) rand & 0.065 & 9 & 3.674 \\
\hline (8) SRAT Volume (gals) rand & 3900 & 1 & 1 \\
\hline (9) Frit 320 Aluminum wt $\%(v)$ rand & 0.24907861 & 2 & 2 \\
\hline (9) Frit 320 Boron $w t \%(v)$ rand & 2.47043302 & 1.1 & 1.1 \\
\hline (9) Frit 320 Calcium wt $\%(v)$ rand & 0.10720514 & 2 & 2 \\
\hline (9) Frit 320 Chromium wt $\%$ (v) rand & 0.0013684 & 2 & 2 \\
\hline (9) Frit 320 Copper wt\% (v) rand & 0 & 2 & 2 \\
\hline$(9)$ Frit 320 Iron wt $\%(v)$ rand & 0.03326678 & 2 & 2 \\
\hline (9) Frit 320 Potassium wt $\%$ (v) rand & 0.01302293 & 2 & 2 \\
\hline (9) Frit 320 Lithium $w t \%(v)$ rand & 3.77056582 & 1.2 & 1.2 \\
\hline (9) Frit 320 Magnesium wt\% (v) rand & 0.03501377 & 2 & 2 \\
\hline (9) Frit 320 Manganese $w t \%(v)$ rand & 0.00077446 & 2 & 2 \\
\hline (9) Frit 320 Sodium $w t \%(v)$ rand & 8.94772848 & 1 & 1 \\
\hline (9) Frit 320 Nickel wt $\%$ (v) rand & 0.00088404 & 2 & 2 \\
\hline (9) Frit 320 Silicon $w t \%(v)$ rand & 33.1835196 & 0.5 & 0.5 \\
\hline (9) Frit 320 Titanium wt $\%(v)$ rand & 0.03615785 & 2 & 2 \\
\hline (9) Frit 320 Uranium wt\% (v) rand & 0 & - & - \\
\hline (9) Frit 320 Zirconium wt $\%$ (v) rand & 0.00666278 & 2 & 2 \\
\hline (10) Frit 320 (lbs) rand & 13000 & 1 & 1 \\
\hline (11) Frit lbs from 5 Can Decon rand & 1000 & 2.5 & 2.5 \\
\hline
\end{tabular}


WSRC-TR-2004-00508

Revision 0

Table A3. Estimated WLs for Batches 234 through 265

\begin{tabular}{|c|c|c|c|c|c|}
\hline Batch & $\begin{array}{c}\mathbf{L i} \\
\text { (wt\%) }\end{array}$ & $\begin{array}{l}\text { Sum of } \\
\text { Oxides }\end{array}$ & $\begin{array}{c}\begin{array}{c}\mathrm{Li} 20 \\
(\mathrm{wt} \%)\end{array} \\
\end{array}$ & $\begin{array}{c}\text { Li2O (wt\%) } \\
\text { in Frit }\end{array}$ & $\begin{array}{c}\% \\
\text { WL } \\
\end{array}$ \\
\hline 234 & 2.296 & 97.460 & 4.943 & 7.980 & 36.44 \\
\hline 234 & 2.259 & 96.300 & \begin{tabular}{|l|l|}
4.863 \\
\end{tabular} & 7.980 & 36.72 \\
\hline 234 & 2.262 & 96.020 & \begin{tabular}{|l|l|}
4.870 \\
\end{tabular} & 7.980 & 36.45 \\
\hline 234 & 2.268 & 96.110 & \begin{tabular}{|l|l|}
4.883 \\
\end{tabular} & 7.980 & 36.33 \\
\hline 235 & 2.269 & 97.250 & \begin{tabular}{|l}
4.885 \\
\end{tabular} & 8.110 & 38.06 \\
\hline 235 & 2.300 & 97.520 & \begin{tabular}{|l|}
4.952 \\
\end{tabular} & 8.110 & 37.39 \\
\hline 235 & 2.275 & 997.350 & 4.898 & 8.110 & 37.96 \\
\hline 235 & 2.295 & 96.990 & \begin{tabular}{|l|}
4.941 \\
\end{tabular} & 8.110 & 37.19 \\
\hline 236 & 2.302 & 98.170 & \begin{tabular}{|l|l}
4.956 \\
\end{tabular} & 8.190 & 38.36 \\
\hline 236 & \begin{tabular}{|l|}
2.304 \\
\end{tabular} & $\begin{array}{l}98.370 \\
\end{array}$ & \begin{tabular}{|l|l}
4.960 \\
\end{tabular} & 8.190 & 38.43 \\
\hline 236 & \begin{tabular}{|l|}
2.339 \\
\end{tabular} & 98.190 & \begin{tabular}{|l|l|}
5.036 \\
\end{tabular} & 8.190 & 37.38 \\
\hline 236 & 2.261 & $\begin{array}{l}97.470 \\
\end{array}$ & \begin{tabular}{|l|l|}
4.868 \\
\end{tabular} & 8.190 & 39.02 \\
\hline 237 & 2.208 & 98.430 & \begin{tabular}{|l|l|}
4.754 \\
\end{tabular} & 8.180 & 40.96 \\
\hline 237 & 2.263 & $\begin{array}{l}97.540 \\
\end{array}$ & \begin{tabular}{|l|}
4.872 \\
\end{tabular} & 8.180 & 38.94 \\
\hline 237 & 2.286 & 97.360 & \begin{tabular}{|l|}
4.922 \\
\end{tabular} & 8.180 & 38.20 \\
\hline 237 & 2.343 & 98.420 & 5.044 & 8.180 & 37.34 \\
\hline 238 & 2.356 & 96.110 & 5.072 & 8.180 & 35.49 \\
\hline 238 & \begin{tabular}{|l|}
2.339 \\
\end{tabular} & 96.010 & \begin{tabular}{|l|}
5.036 \\
\end{tabular} & 8.180 & 35.88 \\
\hline 238 & 2.352 & 96.270 & \begin{tabular}{|l|l|}
5.064 \\
\end{tabular} & 8.180 & 35.70 \\
\hline 238 & 2.339 & 96.460 & 5.036 & 8.180 & 36.18 \\
\hline 239 & 2.388 & 997.640 & 5.141 & 8.120 & 35.15 \\
\hline 239 & 2.414 & 98.050 & \begin{tabular}{|l|l}
5.197 \\
\end{tabular} & 8.120 & 34.72 \\
\hline 239 & 2.415 & $\begin{array}{l}97.470 \\
\end{array}$ & \begin{tabular}{|l|l|}
5.199 \\
\end{tabular} & 8.120 & 34.31 \\
\hline 239 & 2.407 & $\begin{array}{l}95.580 \\
\end{array}$ & \begin{tabular}{|l|l|}
5.182 \\
\end{tabular} & 8.120 & 33.23 \\
\hline 240 & 2.203 & 98.460 & \begin{tabular}{|l|l|}
4.743 \\
\end{tabular} & 8.190 & 41.19 \\
\hline 240 & 2.259 & 96.800 & \begin{tabular}{|l|l|}
4.863 \\
\end{tabular} & 8.190 & 38.65 \\
\hline 240 & 2.200 & $\begin{array}{l}96.740 \\
\end{array}$ & \begin{tabular}{|l|l}
4.736 \\
\end{tabular} & 8.190 & 40.22 \\
\hline 240 & 2.208 & 95.930 & \begin{tabular}{|l|l|}
4.754 \\
\end{tabular} & 8.190 & 39.50 \\
\hline 241 & 2.358 & 97.450 & \begin{tabular}{|l|l}
5.077 \\
\end{tabular} & 8.270 & 37.01 \\
\hline 241 & 2.416 & 99.210 & \begin{tabular}{|l|}
5.201 \\
\end{tabular} & 8.270 & 36.60 \\
\hline 241 & 2.485 & 98.530 & \begin{tabular}{|l|l|}
5.350 \\
\end{tabular} & 8.270 & 34.34 \\
\hline 241 & \begin{tabular}{|l|}
2.439 \\
\end{tabular} & 93.320 & \begin{tabular}{|l|}
5.251 \\
\end{tabular} & 8.270 & 31.96 \\
\hline 242 & 2.182 & 997.170 & \begin{tabular}{|l|l|}
4.698 \\
\end{tabular} & 8.100 & 40.32 \\
\hline 242 & 2.243 & 96.420 & \begin{tabular}{|l|l|}
4.829 \\
\end{tabular} & 8.100 & 38.17 \\
\hline 242 & 2.188 & 96.420 & \begin{tabular}{|l|l}
4.711 \\
\end{tabular} & 8.100 & 39.69 \\
\hline 242 & 2.205 & 96.620 & \begin{tabular}{|l|l}
4.747 \\
\end{tabular} & 8.100 & 39.34 \\
\hline 243 & \begin{tabular}{|l|}
2.478 \\
\end{tabular} & 99.760 & 5.335 & 8.110 & 34.06 \\
\hline 243 & 2.468 & 997.550 & \begin{tabular}{|l|l|}
5.313 \\
\end{tabular} & 8.110 & 32.84 \\
\hline 243 & \begin{tabular}{|l|}
2.320 \\
\end{tabular} & 95.210 & \begin{tabular}{|l|l|}
4.995 \\
\end{tabular} & 8.110 & 35.31 \\
\hline 243 & 2.456 & $\begin{array}{l}94.470 \\
\end{array}$ & 5.288 & 8.110 & 30.99 \\
\hline 244 & 2.375 & 96.700 & \begin{tabular}{|l|l|}
5.113 \\
\end{tabular} & 8.163 & 35.22 \\
\hline 244 & 2.311 & $\begin{array}{l}96.840 \\
\end{array}$ & \begin{tabular}{|l|l|}
4.975 \\
\end{tabular} & 8.163 & 37.06 \\
\hline 244 & 2.296 & 95.900 & \begin{tabular}{|l|l|}
4.943 \\
\end{tabular} & 8.163 & 36.85 \\
\hline 244 & 2.242 & $\begin{array}{l}94.380 \\
\end{array}$ & \begin{tabular}{|l|l}
4.827 \\
\end{tabular} & 8.163 & 37.35 \\
\hline 245 & 2.254 & 98.580 & \begin{tabular}{|l|l|}
4.853 \\
\end{tabular} & 8.125 & 39.41 \\
\hline 245 & 2.258 & 98.090 & \begin{tabular}{|l|l|}
4.861 \\
\end{tabular} & 8.125 & 39.01 \\
\hline 245 & 2.266 & 97.430 & \begin{tabular}{|l|l|}
4.878 \\
\end{tabular} & 8.125 & 38.37 \\
\hline 245 & 2.238 & 96.690 & 4.818 & 8.125 & 38.67 \\
\hline 246 & \begin{tabular}{|l|}
2.377 \\
\end{tabular} & 98.330 & 5.117 & 8.220 & 36.69 \\
\hline 246 & \begin{tabular}{|l|}
2.309 \\
\end{tabular} & 99.500 & \begin{tabular}{|l|}
4.971 \\
\end{tabular} & 8.220 & 39.22 \\
\hline 246 & 2.405 & 101.810 & \begin{tabular}{|l|l|}
5.178 \\
\end{tabular} & 8.220 & 38.13 \\
\hline 246 & 2.150 & 95.640 & \begin{tabular}{|l|l|}
4.629 \\
\end{tabular} & 8.220 & 41.12 \\
\hline
\end{tabular}


WSRC-TR-2004-00508

Revision 0

Table A3. Estimated WLs for Batches 234 through 265

\begin{tabular}{|c|c|c|c|c|c|}
\hline Batch & \begin{tabular}{|c}
$\mathbf{L i}$ \\
$(\mathrm{wt} \%)$
\end{tabular} & $\begin{array}{l}\text { Sum of } \\
\text { Oxides }\end{array}$ & \begin{tabular}{|l|}
$\begin{array}{c}\text { Li2O } \\
(w t \%)\end{array}$ \\
\end{tabular} & $\begin{array}{c}\mathrm{Li2O}(\mathrm{wt} \%) \\
\text { in Frit }\end{array}$ & $\begin{array}{c}\% \\
\text { WL }\end{array}$ \\
\hline 247 & 2.548 & 97.780 & 5.486 & 8.153 & 31.19 \\
\hline 247 & 2.496 & 96.620 & 5.374 & 8.153 & 31.78 \\
\hline 247 & 2.564 & 98.050 & 5.520 & 8.153 & 30.95 \\
\hline 247 & 2.439 & 96.050 & 5.251 & 8.153 & 32.94 \\
\hline 248 & 2.446 & 97.790 & 5.266 & 8.027 & 32.91 \\
\hline 248 & 2.435 & 97.250 & 5.242 & 8.027 & 32.84 \\
\hline 248 & 2.495 & 97.310 & 5.371 & 8.027 & 31.23 \\
\hline 248 & 2.493 & 97.760 & 5.367 & 8.027 & 31.60 \\
\hline 249 & 2.454 & 98.350 & 5.283 & 8.088 & 33.59 \\
\hline 249 & 2.423 & 97.220 & 5.216 & 8.088 & 33.66 \\
\hline 249 & 2.378 & 97.060 & 5.120 & 8.088 & 34.78 \\
\hline 249 & 2.386 & 96.550 & 5.137 & 8.088 & 34.22 \\
\hline 250 & 2.534 & 97.600 & 5.455 & 8.167 & 31.56 \\
\hline 250 & 2.539 & 96.220 & 5.466 & 8.167 & 30.44 \\
\hline 250 & 2.552 & 97.850 & 5.494 & 8.167 & 31.24 \\
\hline 250 & 2.503 & 94.440 & 5.389 & 8.167 & 30.13 \\
\hline 251 & 2.403 & 96.900 & 5.173 & 7.885 & 32.29 \\
\hline 251 & 2.378 & 96.580 & 5.120 & 7.885 & 32.77 \\
\hline 251 & 2.421 & 96.510 & 5.212 & 7.885 & 31.50 \\
\hline 251 & 2.359 & 95.890 & 5.079 & 7.885 & 32.83 \\
\hline 252 & 2.443 & 97.370 & 5.260 & 8.148 & 33.71 \\
\hline 252 & 2.432 & 96.950 & 5.236 & 8.148 & 33.72 \\
\hline 252 & 2.425 & 96.920 & 5.221 & 8.148 & 33.89 \\
\hline 252 & 2.406 & 96.200 & 5.180 & 8.148 & 33.92 \\
\hline 253 & 2.535 & 98.890 & 5.458 & 8.028 & 31.25 \\
\hline 253 & 2.603 & 97.430 & 5.604 & 8.028 & 28.35 \\
\hline 253 & 2.509 & 95.210 & 5.402 & 8.028 & 29.33 \\
\hline 253 & 2.504 & 94.170 & 5.391 & 8.028 & 28.69 \\
\hline 254 & 2.355 & 97.380 & 5.070 & .238 & 36.80 \\
\hline 254 & 2.366 & 95.870 & 5.094 & 8.238 & 35.50 \\
\hline 254 & 2.418 & 95.120 & 5.206 & 8.238 & 33.57 \\
\hline 254 & 2.408 & 95.420 & 5.184 & 8.238 & 34.05 \\
\hline 255 & 2.519 & 97.600 & 5.423 & 8.170 & 31.99 \\
\hline 255 & 2.470 & 96.400 & 5.318 & 8.170 & 32.48 \\
\hline 255 & 2.481 & 95.170 & 5.341 & 8.170 & 31.30 \\
\hline 255 & 2.456 & 97.920 & 5.288 & 8.170 & 33.91 \\
\hline 256 & 2.503 & 96.730 & 5.389 & 8.158 & 31.71 \\
\hline 256 & 2.367 & 96.200 & 5.096 & 8.158 & 35.06 \\
\hline 256 & 2.343 & 97.040 & 5.044 & 8.158 & 36.29 \\
\hline 256 & 2.355 & 95.470 & 5.070 & 8.158 & 34.90 \\
\hline 257 & 2.450 & 95.610 & 5.275 & 7.982 & 30.88 \\
\hline 257 & 2.457 & 95.440 & 5.290 & 7.982 & 30.56 \\
\hline 257 & 2.496 & 95.590 & 5.374 & 7.982 & 29.57 \\
\hline 257 & 2.455 & 94.710 & 5.285 & 7.982 & 30.09 \\
\hline 258 & 2.396 & 97.340 & 5.158 & 8.015 & 33.88 \\
\hline 258 & 2.324 & 95.580 & 5.003 & 8.015 & 34.69 \\
\hline 258 & 2.397 & 96.970 & 5.161 & 8.015 & 33.60 \\
\hline 258 & 2.398 & 96.130 & 5.163 & 8.015 & 33.00 \\
\hline 259 & 2.309 & 97.740 & 4.971 & 8.070 & 36.97 \\
\hline 259 & 2.263 & 97.690 & 4.872 & 8.070 & 38.20 \\
\hline 259 & 2.314 & 95.710 & 4.982 & 8.070 & 35.50 \\
\hline 259 & 2.225 & 94.020 & 4.790 & 8.070 & 36.87 \\
\hline 260 & 2.389 & 96.530 & 5.143 & 8.070 & 33.98 \\
\hline
\end{tabular}


WSRC-TR-2004-00508

Revision 0

Table A3. Estimated WLs for Batches 234 through 265

\begin{tabular}{||c|c|c|c|c|c||}
\hline Batch & $\begin{array}{c}\text { Li } \\
\text { (wt\%) }\end{array}$ & $\begin{array}{c}\text { Sum of } \\
\text { Oxides }\end{array}$ & $\begin{array}{c}\text { Li2O } \\
\text { (wt\%) }\end{array}$ & $\begin{array}{c}\text { Li2O (wt\%) } \\
\text { in Frit }\end{array}$ & $\begin{array}{c}\text { \% } \\
\text { WL }\end{array}$ \\
\hline 260 & 2.338 & 96.110 & 5.033 & 8.070 & 35.10 \\
\hline 260 & 2.356 & 95.060 & 5.072 & 8.070 & 33.88 \\
\hline 260 & 2.324 & 94.150 & 5.003 & 8.070 & 34.15 \\
\hline 261 & 2.323 & 98.170 & 5.001 & 8.010 & 36.40 \\
\hline 261 & 2.377 & 96.680 & 5.117 & 8.010 & 33.91 \\
\hline 261 & 2.334 & 96.420 & 5.025 & 8.010 & 34.94 \\
\hline 261 & 2.362 & 97.300 & 5.085 & 8.010 & 34.75 \\
\hline 262 & 2.398 & 95.830 & 5.163 & 8.127 & 33.71 \\
\hline 262 & 2.387 & 95.880 & 5.139 & 8.127 & 34.05 \\
\hline 262 & 2.437 & 95.480 & 5.247 & 8.127 & 32.39 \\
\hline 262 & 2.427 & 94.040 & 5.225 & 8.127 & 31.63 \\
\hline 263 & 2.412 & 96.000 & 5.193 & 7.978 & 32.20 \\
\hline 263 & 2.419 & 95.860 & 5.208 & 7.978 & 31.91 \\
\hline 263 & 2.394 & 95.130 & 5.154 & 7.978 & 32.09 \\
\hline 263 & 2.417 & 94.670 & 5.204 & 7.978 & 31.11 \\
\hline 264 & 2.378 & 98.410 & 5.120 & 7.982 & 34.82 \\
\hline 264 & 2.353 & 96.520 & 5.066 & 7.982 & 34.24 \\
\hline 264 & 2.389 & 96.540 & 5.143 & 7.982 & 33.25 \\
\hline 264 & 2.380 & 95.940 & 5.124 & 7.982 & 33.09 \\
\hline 265 & 2.434 & 97.120 & 5.240 & 7.906 & 31.75 \\
\hline 265 & 2.456 & 97.850 & 5.288 & 7.906 & 31.65 \\
\hline 265 & 2.433 & 97.840 & 5.238 & 7.906 & 32.28 \\
\hline 265 & 2.376 & 95.140 & 5.115 & 7.906 & 32.00 \\
\hline
\end{tabular}


WSRC-TR-2004-00508

Revision 0

Table A4. Nominal Values and Uncertainties for Inputs to the Estimation of WL for SME Batch 261

\begin{tabular}{||c|c|c|c|c|c||}
\hline Element & Sample 1 & Sample 2 & Sample 3 & Sample 4 & \%RSD \\
\hline Aluminum & 2.59 & 2.681 & 2.802 & 2.685 & 4.1 \\
\hline Boron & 1.489 & 1.46 & 1.388 & 1.458 & 3.5 \\
\hline Calcium & 1.118 & 1.015 & 0.975 & 0.984 & 6 \\
\hline Chromium & 0.092 & 0.074 & 0.074 & 0.077 & 17.1 \\
\hline Copper & 0.026 & 0.024 & 0.025 & 0.024 & 15.2 \\
\hline Iron & 10.654 & 9.722 & 10.018 & 10.105 & 3.3 \\
\hline Potassium & 0.159 & 0.123 & 0.453 & 0.14 & 20 \\
\hline Lithium & 2.323 & 2.377 & 2.334 & 2.362 & 2.1 \\
\hline Magnesium & 0.905 & 0.815 & 0.773 & 0.794 & 3.5 \\
\hline Manganese & 1.417 & 1.278 & 1.256 & 1.275 & 3.8 \\
\hline Sodium & 8.673 & 8.586 & 8.387 & 8.576 & 6 \\
\hline Nickel & 0.58 & 0.498 & 0.496 & 0.52 & 17.7 \\
\hline Silicon & 21.5525 & 21.8955 & 21.535 & 21.916 & 2.1 \\
\hline Titanium & 0.034 & 0.033 & 0.031 & 0.033 & 25 \\
\hline Uranium & 3.657 & 3.386 & 3.569 & 3.474 & 9.5 \\
\hline Zirconium & 0.066 & 0.065 & 0.068 & 0.066 & 15.6 \\
\hline \hline \multicolumn{2}{|c|}{ Frit Li $\mathbf{0}$} & Nominal & $\mathbf{8 . 0 1}$ & \%RSD & $\mathbf{0 . 2 5}$ \\
\hline \hline
\end{tabular}


Exhibit A1. Overview of Impact of Uncertainties on Measured WLs

(assuming analytical uncertainties with only 1 sample)

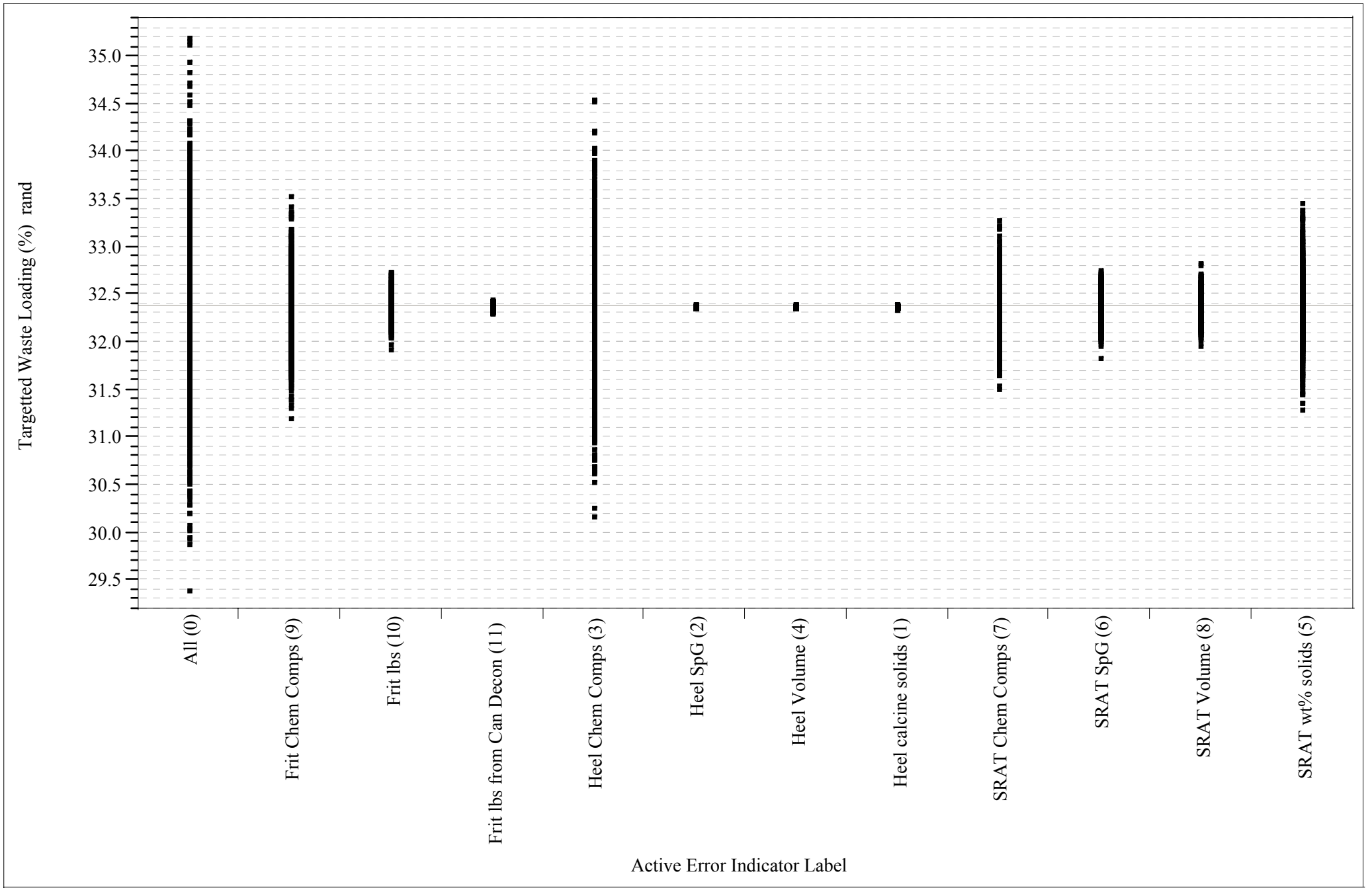


WSRC-TR-2004-00508

Revision 0

Exhibit A2. Histograms and Other Descriptive Statistics for WLs with Analytical Uncertainties Assuming 1 Sample

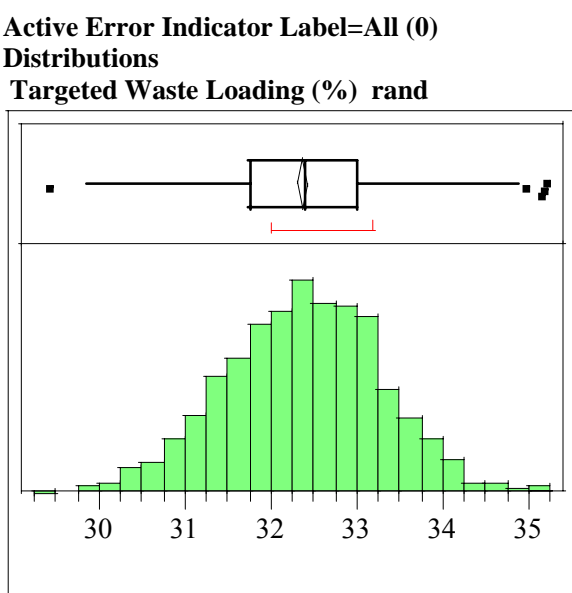

Quantiles

$97.5 \%$

(5.0\%

quartile

median

$25.0 \%$ quartile

$10.0 \%$

$2.5 \%$

$0.5 \%$

$0.0 \%$

minimum

Mean

Std Dev

Std Err Mean

upper $95 \%$ Mean

lower $95 \%$ Mean

$\mathrm{N}$

34.059

33.506

33.002

32.405

31.757

31.203

30.558

30.037
29.405

32.373504

0.8988659

0.0284246

32.429283

32.317726

1000
Active Error Indicator Label=Frit Chem Comps (9) Distributions

Targeted Waste Loading (\%) rand

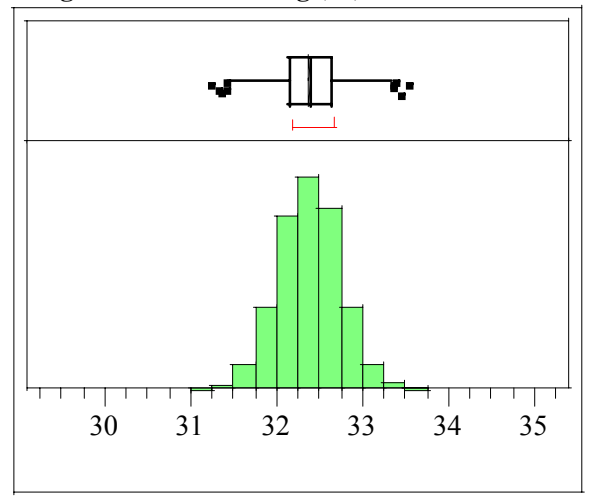

Quantiles

$100.0 \%$ maximum

$99.5 \%$

$97.5 \%$

$90.0 \%$

$75.0 \%$

$50.0 \%$

$25.0 \%$

(n)

quartile

median

quartile

$10.0 \%$

$2.5 \%$

$0.5 \%$

$0.0 \%$

minimum

Mean

Std Dev

Std Err Mean

upper $95 \%$ Mean

lower $95 \%$ Mean

$\mathrm{N}$

32.40805
32.363986

32.363986
Active Error Indicator Label=Frit lbs (10)

Distributions

Targeted Waste Loading (\%) rand

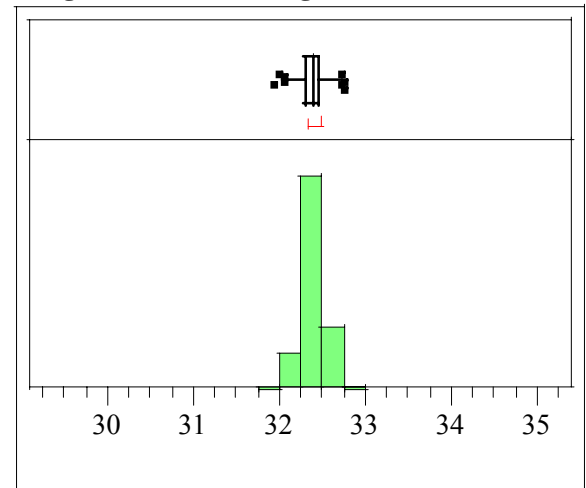

Quantiles

32.752

$99.5 \%$

$97.5 \%$

$\begin{array}{ll}90.0 \% & \\ 75.0 \% & \text { quartile }\end{array}$

$50.0 \%$ median 32.398

$25.0 \%$ quartile $\quad 32.312$

$\begin{array}{ll}10.5 \% & 32.153\end{array}$

$0.5 \% \quad 32.071$

$0.0 \%$ minimum 31.93

Moments

Mean

32.396082

Std Dev

Std Err Mean

upper $95 \%$ Mean

lower $95 \%$ Mean

0.1224269

0.0038715

32.403679
$\mathrm{N}$ 
WSRC-TR-2004-00508

Revision 0

Exhibit A2. Histograms and Other Descriptive Statistics for WLs with Analytical Uncertainties Assuming 1 Sample

Active Error Indicator Label=Frit lbs from Can Decon (11)

Distributions

Targeted Waste Loading (\%) rand

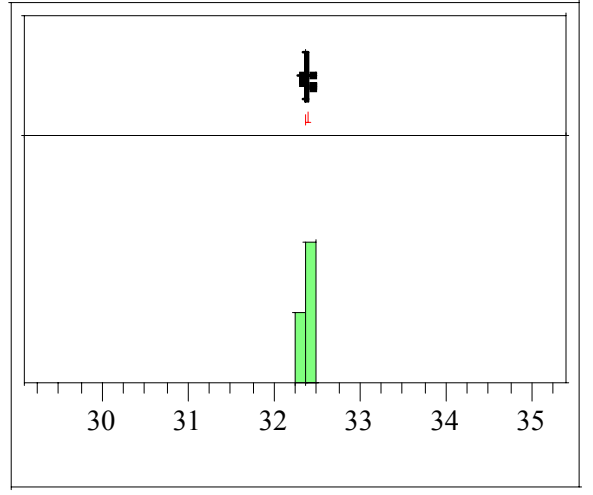

\section{Quantiles}

$100.0 \% \quad$ maximum

$99.5 \%$

$97.5 \%$

$90.0 \%$

$75.0 \%$

quartile

$50.0 \%$ median

$25.0 \%$ quartile

$10.0 \%$

$2.5 \%$

$0.5 \%$

$0.0 \%$

Moments

minimum

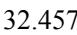

32.450

32.434

32.418

32.402

32.386

32.370

32.355

32.341

32.327

Mean

Std Dev

Std Err Mean

upper $95 \%$ Mean

lower 95\% Mean

32.385901

0.0239868

0.0007585

32.38739

32.384413

1000
Active Error Indicator Label=Heel Chem Comps (3) Distributions

Targeted Waste Loading (\%) rand

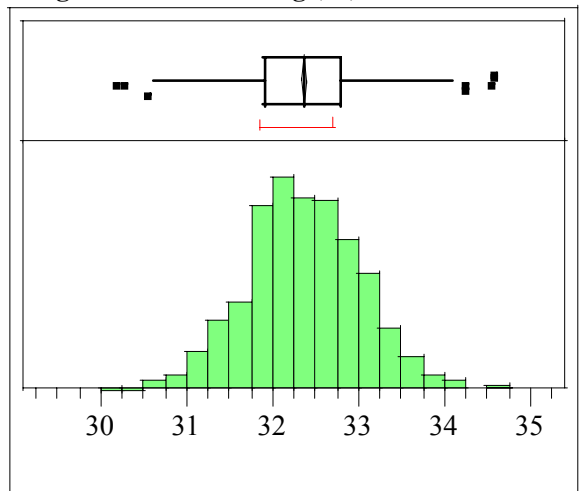

Quantiles

$100.0 \%$ maximum

$99.5 \%$

$97.5 \%$

$90.0 \%$

$75.0 \%$

$50.0 \%$

(n)

$25.0 \%$

quartile

median

quartile

$10.0 \%$

$2.5 \%$

$0.5 \%$

Moments

minimum

Std Dev

Std Err Mean

upper $95 \%$ Mean

lower $95 \%$ Mean

$\mathrm{N}$

34.567

34.222

33.662

33.198

32.794

32.363

31.930

31.539

31.073

30.651

30.177

32.368519

0.6613395

0.0209134

32.409558

32.32748
1000
Active Error Indicator Label=Heel SpG (2)

Distributions

Targeted Waste Loading (\%) rand

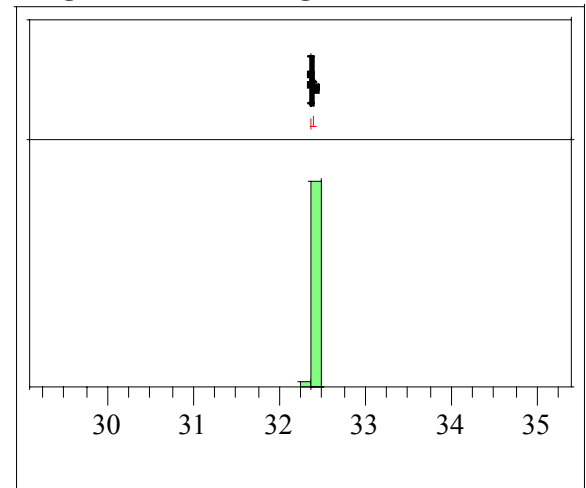

Quantiles

$100.0 \% \quad$ maximum $\quad 32.408$

$99.5 \%-32.402$

$\begin{array}{ll}97.5 \% & 32.397\end{array}$

$90.0 \%$

$\begin{array}{ll}75.0 \% & \text { quartile } \\ 50.0 \% & 32.390\end{array}$

32.386

$25.0 \%$ quartile

$\begin{array}{ll}10.0 \% & 32.378 \\ 2.5 \% & 32.374\end{array}$

$\begin{array}{ll}0.5 \% & 32.371\end{array}$

$0.0 \%$ minimum 32.368

Moments

Mean

Std Dev

Std Err Mean

upper $95 \%$ Mean

lower $95 \%$ Mea

32.386068

0.0059989

0.0001897

32.38644

32.385696 
Revision 0

Exhibit A2. Histograms and Other Descriptive Statistics for WLs with Analytical Uncertainties Assuming 1 Sample

Active Error Indicator Label=Heel Volume (4) Distributions

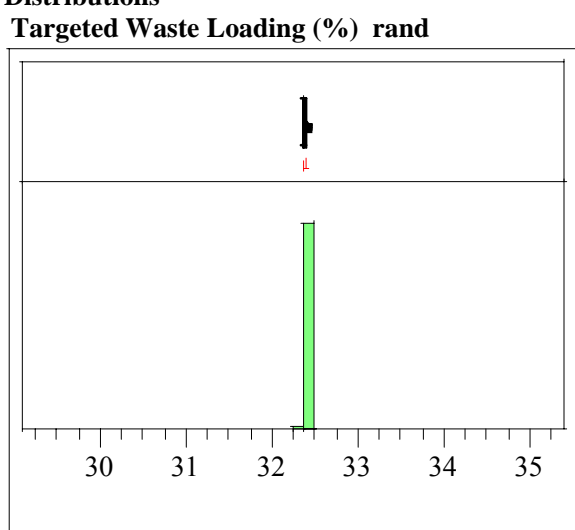

Quantiles

$100.0 \% \quad$ maximum

$99.5 \%$

$97.5 \%$

$90.0 \%$

$75.0 \%$

$50.0 \% \quad$ quartile

$25.0 \%$ quartile

$10.0 \%$

$2.5 \%$

$0.5 \%$

$0.0 \%$

Moments

minimum

Mean

Std Dev

Std Err Mean

upper $95 \%$ Mean

lower $95 \%$ Mean

$\mathrm{N}$

2.385729

1000
Active Error Indicator Label=Heel calcine solids (1) Distributions

Targeted Waste Loading (\%) rand

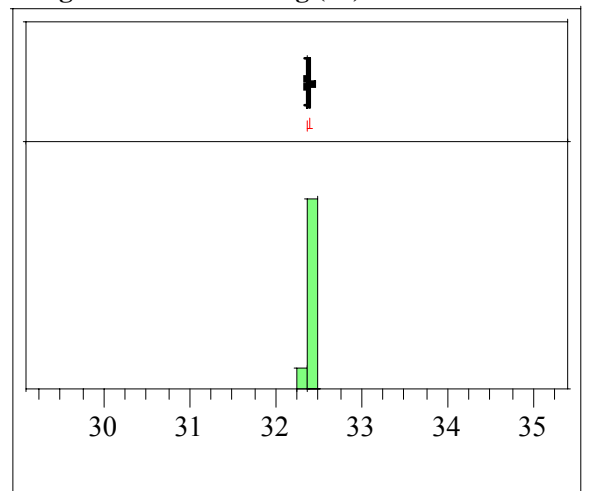

Quantiles

$100.0 \%$ maximum

$99.5 \%$

$97.5 \%$

$90.0 \%$

$75.0 \%$

$50.0 \%$

$25.0 \%$

quarte

quartile

median

quartile

$10.0 \%$

$2.5 \%$

$0.5 \%$

$0.0 \%$

minimum

(Moments

Mean

Std Dev
Std Err Mean

upper $95 \%$ Mean

lower 95\% Mean

$\mathrm{N}$

.38527
1000
Active Error Indicator Label=SRAT Chem Comps (7) Distributions

Targeted Waste Loading (\%) rand

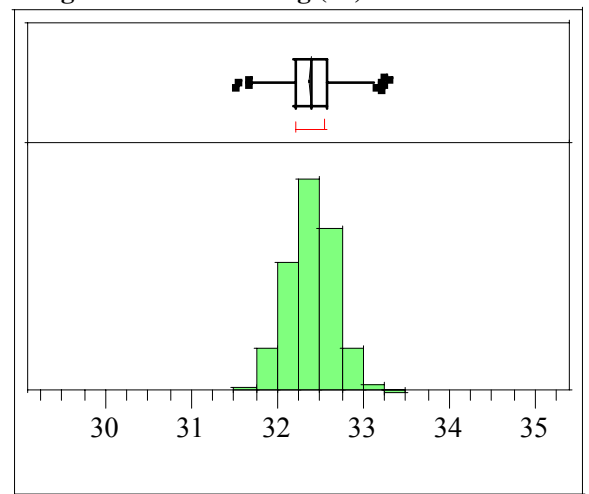

Quantiles

$100.0 \%$ maximum $\quad 33.299$

$99.5 \% \quad 33.213$

$97.5 \% \quad 32.91$

$\begin{array}{ll}90.0 \% & 32.724 \\ 75.0 \% & \text { quartile }\end{array}$

$50.0 \%$ median $\quad 32.390$

$25.0 \%$ quartile $\quad 32.216$
$10.0 \%$

$10.0 \% \quad 32.045$

$2.5 \% \quad 31.854$

$\begin{array}{ll}0.5 \% & 31.705 \\ 0.0 \% & 31.522\end{array}$

$0.0 \% \quad$ minimum 31.522

Moments

Mean

Std Dev

Std Err Mean

upper $95 \%$ Mean

lower $95 \%$ Mea

32.390796

0.2672311

0.0084506

32.407379

32.374213 
Revision 0

Exhibit A2. Histograms and Other Descriptive Statistics for WLs with Analytical Uncertainties Assuming 1 Sample

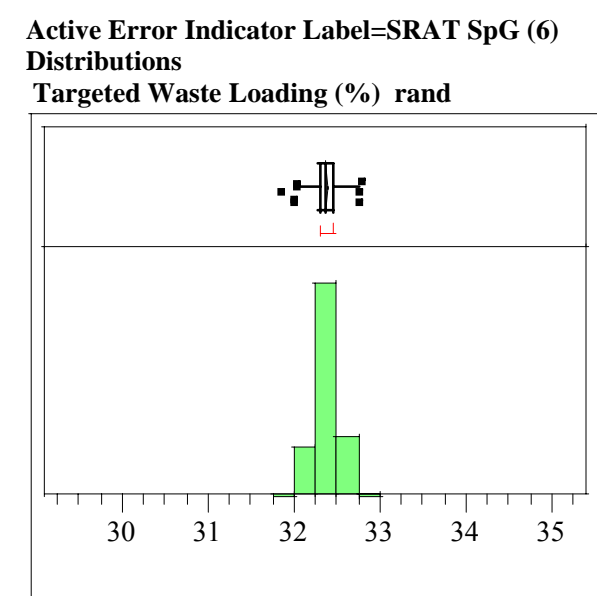

Quantiles

$100.0 \% \quad$ maximum $\quad 32.771$

$97.5 \%$

$90.0 \%$

quartile

median

$25.0 \%$ quartile

$10.0 \%$

$2.5 \%$

$0.5 \%$

Moments

minimum

Mean

Std Dev

Std Err Mean

upper $95 \%$ Mean

lower 95\% Mean

$\mathrm{N}$

32.639

32.469

32.383

32.296

32.212

32.126

32.034

31.854

32.383917

0.1325667

0.0041921

32.392144

32.375691

1000
Active Error Indicator Label=SRAT Volume (8)

Distributions

Targeted Waste Loading (\%) rand

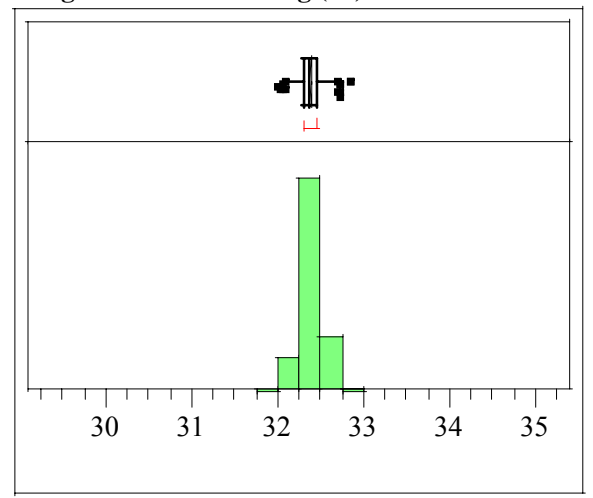

Quantiles

$100.0 \%$ maximum

$99.5 \%$

$97.5 \%$

$90.0 \%$

$75.0 \%$

$50.0 \%$

quartile

median

quartile

$25.0 \%$

$10.0 \%$

$2.5 \%$

$0.5 \%$
$0.0 \%$

Moments

minimum

Mean

Std Dev

Std Err Mean

upper $95 \%$ Mean

lower 95\% Mean

$\mathrm{N}$

32.392537

0.1199607

0.0037935

32.399981
32.385093

1000
Active Error Indicator Label=SRAT wt \% solids (5) Distributions

Targeted Waste Loading (\%) rand

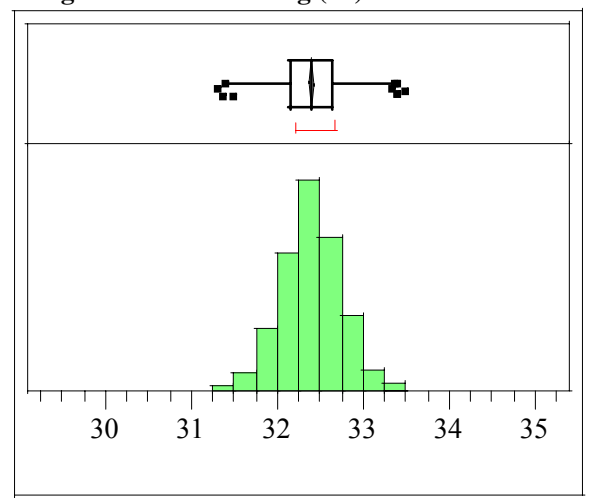

Quantiles

33.477

$99.5 \% \quad 33.354$

$97.5 \% \quad 33.104$

$\begin{array}{ll}90.0 \% & \\ 75.0 \% & \text { quartile }\end{array}$

$50.0 \%$ median 32.398

$25.0 \%$ quartile $\quad 32.166$

$25.0 \%$ quartile

$\begin{array}{ll}10.0 \% & 31.948 \\ 2.5 \% & 31.686\end{array}$

$\begin{array}{ll}0.5 \% & 31.686 \\ 0.0 \% & 31.309\end{array}$

$0.0 \%$ minimum 31.309

Moments

Mean

32.397053

Std Dev

Std Err Mean

upper $95 \%$ Mean

lower $95 \%$ Mean

0.3512802

0.0111085

32.418851

$\mathrm{N}$ 


\section{Exhibit A3. Histograms and Other Descriptive Statistics for Inputs to WL Targeting Assuming 1 Sample}

Active Error Indicator Label=All (0)

Distributions

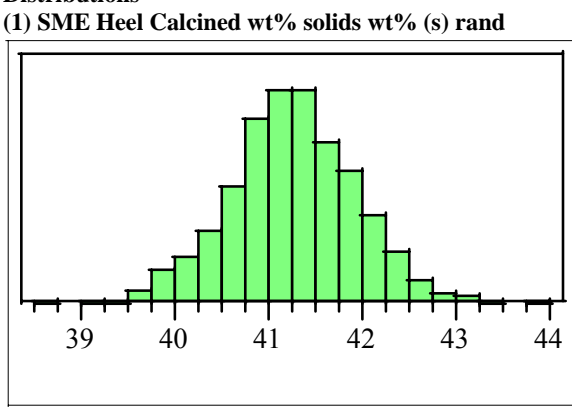

Moments

Mean

Std Dev

(1) $95 \%$ Me

lower $95 \%$ Mean

$$
\begin{aligned}
& 41.263233 \\
& 0.6674493 \\
& 0.0211066 \\
& 41.304652 \\
& 41.221815
\end{aligned}
$$

1000

(2) SME Heel Specific gravity spgr rand

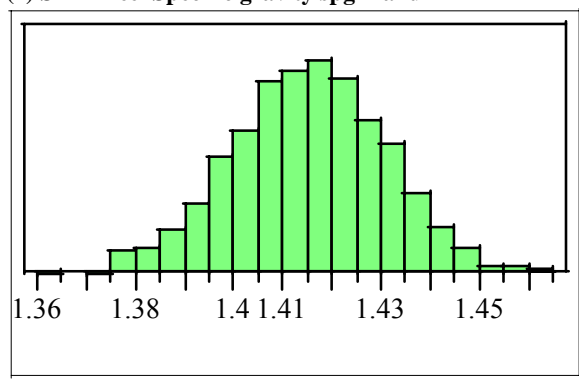

Moments

Mean

Std Dev

upper $95 \% \mathrm{Me}$

lower $95 \%$ Mean

1.4152176

0.0155526

0.0004918

1.4161828

1000

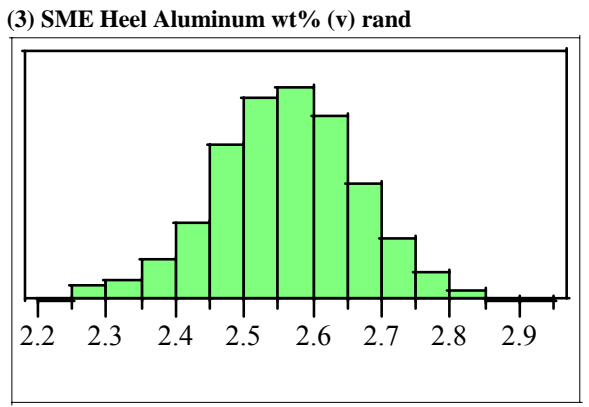

Moments

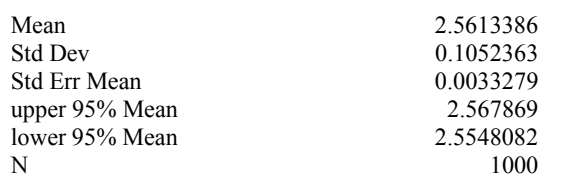

(3) SME Heel Boron wt \% (v) rand

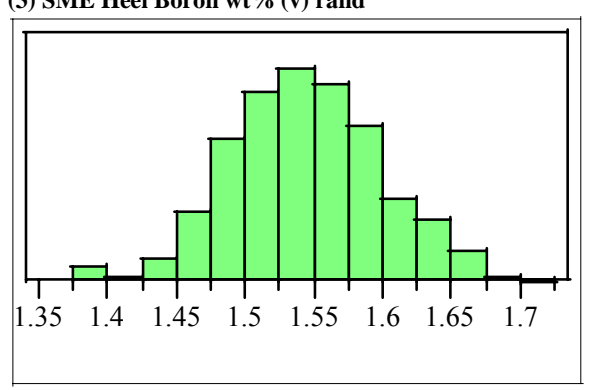

Moments

Mean

Std Err Mean

(1) $95 \%$ Mean

lower $95 \%$ Mean

$\mathrm{N}$

0.0017376

1.5475147

-1000
1000
(3) SME Heel Calcium wt \% (v) rand

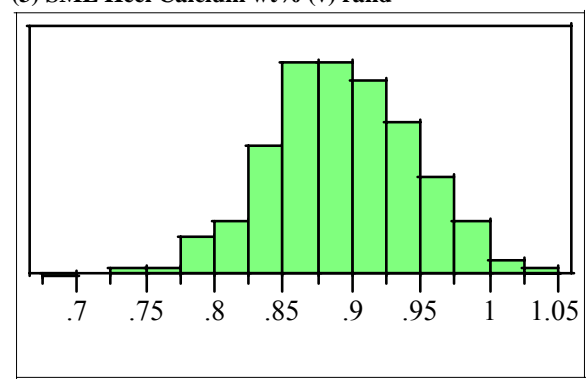

Moments

Mean

Std Err Mean

upper $95 \%$ Mean

lower $95 \%$ Mean

0.8931166

0.0524099

0.0016573

0.896368

(3) SME Heel Chromium wt \% (v) rand

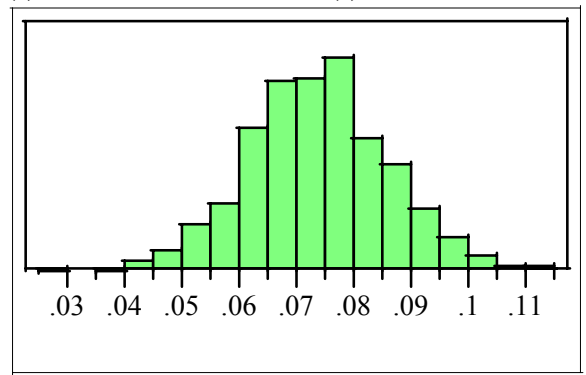

Moments

Mean

Std Err Mean

upper $95 \%$ Mean

lower $95 \%$ Mea

0.0736697

0.0122386

0.0744291

0.0729102 
Exhibit A3. Histograms and Other Descriptive Statistics for Inputs to WL Targeting Assuming 1 Sample

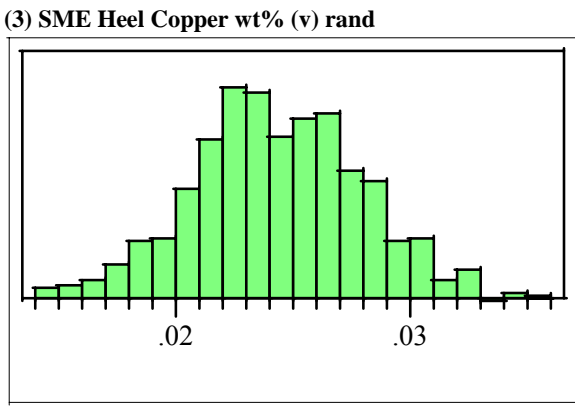

Moments

Mean

Std Dev

Std Err Mean

lower $95 \%$ Mean

0.0243482

0.0036602

0.0001157

0.0245753

(3) SME Heel Iron wt\% (v) rand

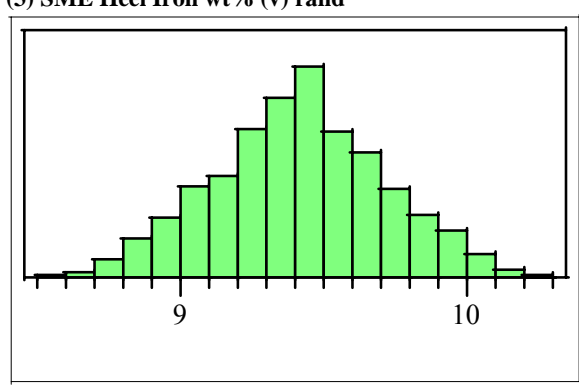

Moments

Mean

Std Dev

upper $95 \% \mathrm{Me}$

lower $95 \%$ Mean

9.4117924

0.3054719

0.0096599

9.4307484

1000
(3) SME Heel Potassium wt\% (v) rand

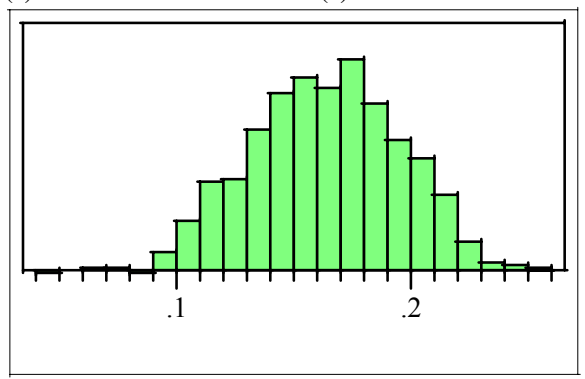

Moments

Mean

Std Err Mean

upper $95 \%$ Mean

upper $95 \%$ Mean

(3) SME Heel Lithium wt\% (v) rand

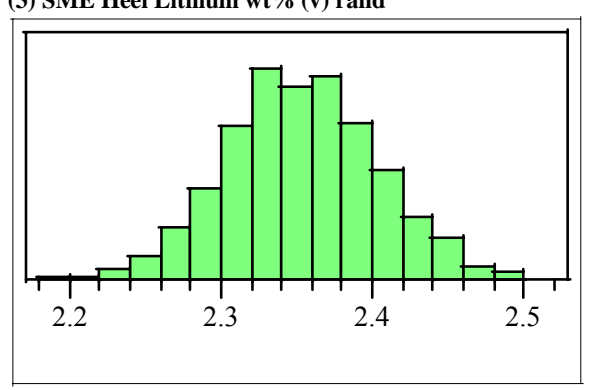

Moments

Mean

Std Dev

upper $95 \%$ Mean

lower $95 \%$ Mean

$95 \%$ Mea

1000
(3) SME Heel Magnesium wt\% (v) rand

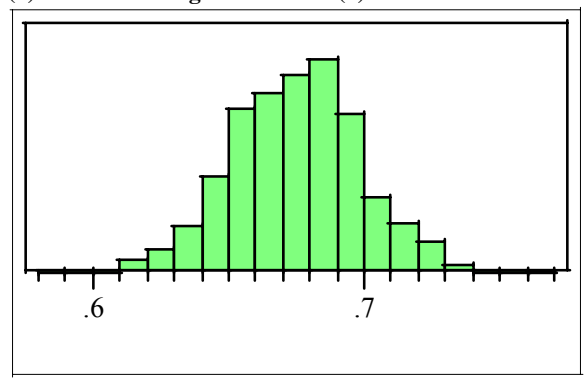

Moments

Mean

Std Err Mean

upper $95 \%$ Mean

lower $95 \%$ Mean

0.6748296

0.0238275

0.0007535

0.676308

(3) SME Heel Manganese wt \% (v) rand

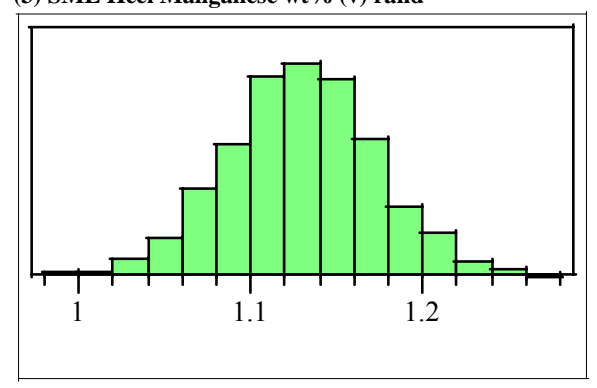

Moments

Mean

Std Err Me

upper $95 \%$ Mean

lower $95 \%$ Mea

1.1293235

0.0435915

0.0013785
1.1320285
1.126184

1.1320285

1000 
Exhibit A3. Histograms and Other Descriptive Statistics for Inputs to WL Targeting Assuming 1 Sample

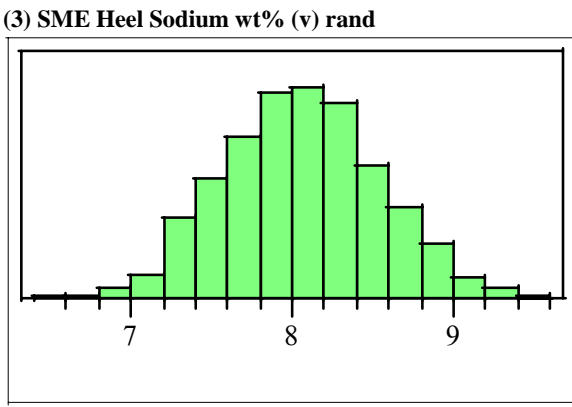

Moments

Mean

Std Err Mea

Std $95 \%$ Mean

lower $95 \%$ Mean

8.0565664

0.4821181

0.0152459

8.0864841

(3) SME Heel Nickel wt\% (v) rand

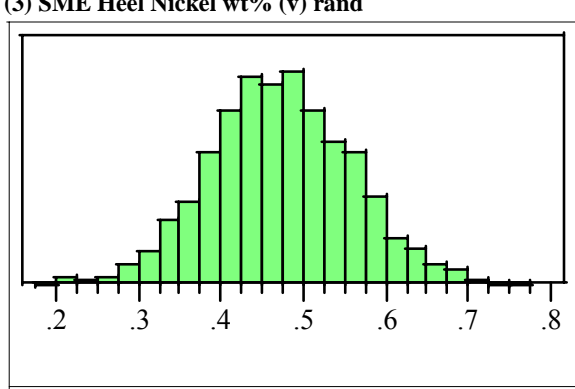

Moments

Mean

Std Dev

lower $95 \%$ Mean

0.4722485

0.0858191

0.0027138
0.477574

0.477574

1000

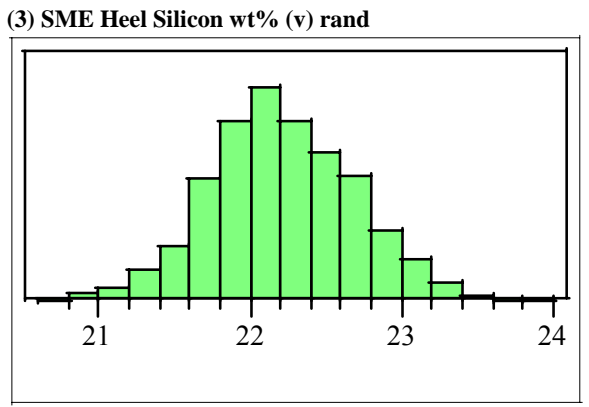

Moments

Mean

Std Err Mean

upper $95 \%$ Mean

upper 95\% Mean

0.0149696

22.239518

N 22.180768

(3) SME Heel Titanium wt\% (v) rand

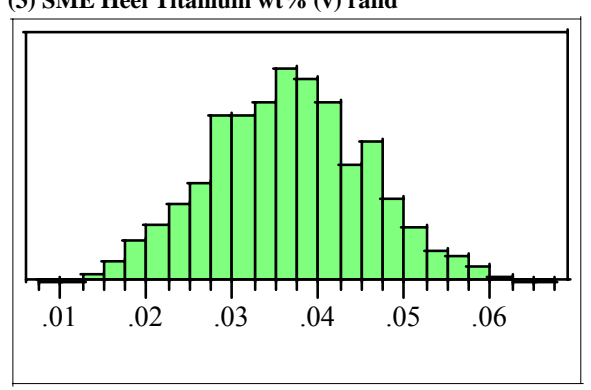

Moments

Mean

Mean
Std Dev

Std Err Mean

upper 95\% Mean

lower $95 \%$ Mea

$95 \%$ Mean

0.0002899

1000
(3) SME Heel Uranium wt\% (v) rand

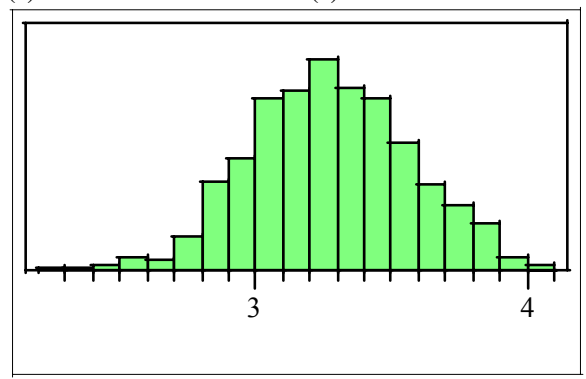

Moments

Mean

Std Err Mean

upper $95 \%$ Mean

lower $95 \%$ Mean

3.2731949

0.3044525

0.0096276

3.2920876

(3) SME Heel Zirconium wt \% (v) rand

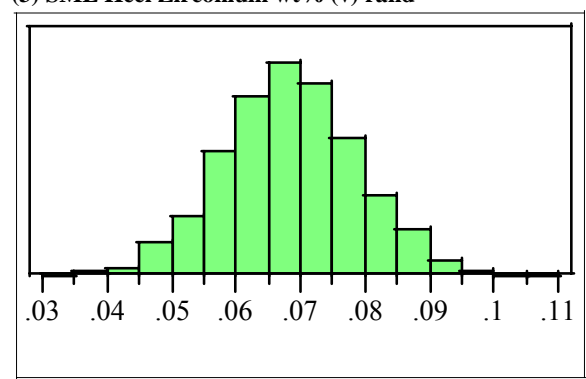

Moments

Mean

Std Err Mean

upper $95 \%$ Mean

lower $95 \%$ Mea

0.0683398

0.0104777

0.0003313
0.06899
0.066896

0.0676896 
Exhibit A3. Histograms and Other Descriptive Statistics for Inputs to WL Targeting Assuming 1 Sample

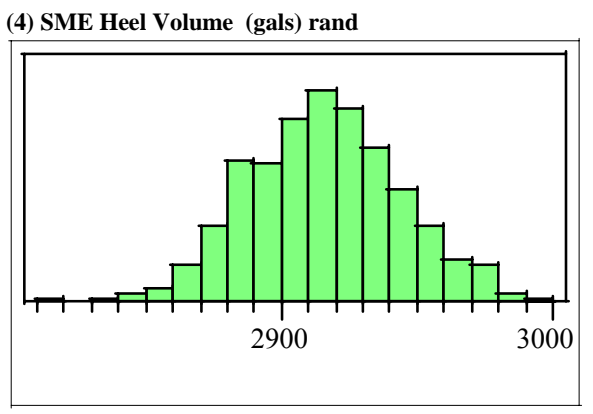

Moments

Mean

Std Dev

upper $95 \%$ Mean

lower $95 \%$ Mean

2915.5008

28.622453

0.9051214

2917.277

1000

(5) SRAT Total wt \% solids wt $\%$ (s) rand

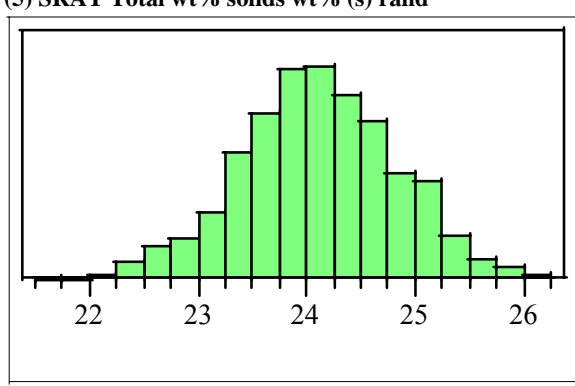

Moments

Mean

Std Dev

upper $95 \%$ Mea

lower $95 \%$ Mean

24.107526

0.7105275

0.0224689

24.151618

1000

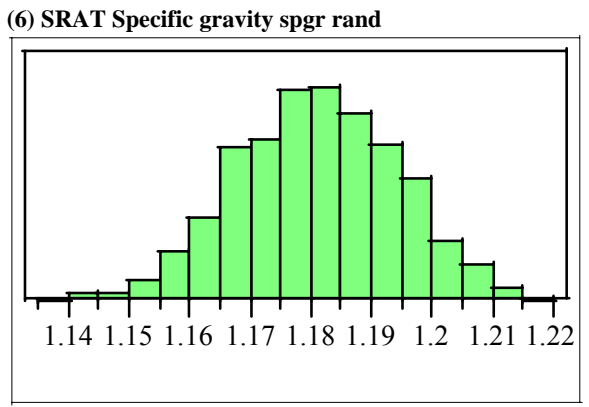

Moments

Mean

Std Err Mean

upper $95 \%$ Mean

upper $95 \%$ Mea

1.1810913

0.0131407

0.000415

1.1819068

1000

(7) SRAT Aluminum wt\% (s) rand

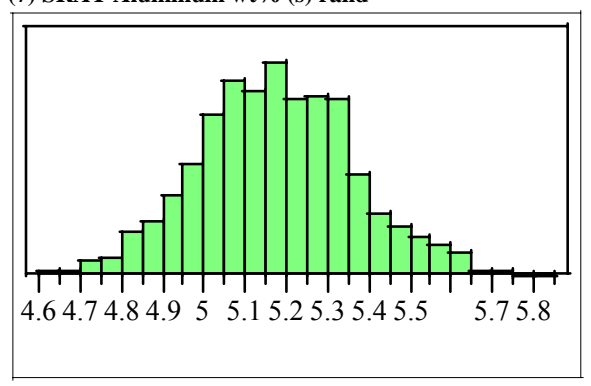

Moments

Mean

Std Dev

upper $95 \%$ Me

upper $95 \%$ Mea

$\mathrm{N}$
(7) SRAT Boron wt\% (s) rand

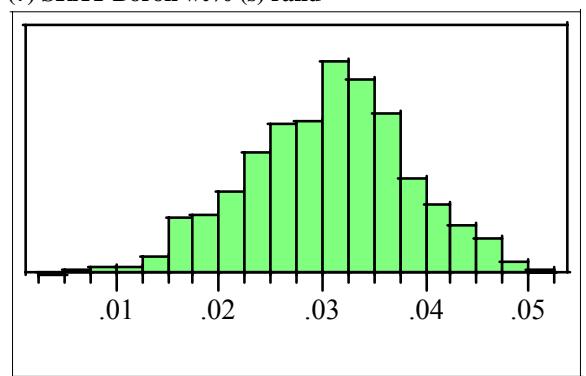

Moments

Mean

Std Err Mean

upper $95 \%$ Mean

lower $95 \%$ Mean

0.0305799

0.0078732

0.031068

0.031068

(7) SRAT Calcium wt \% (s) rand

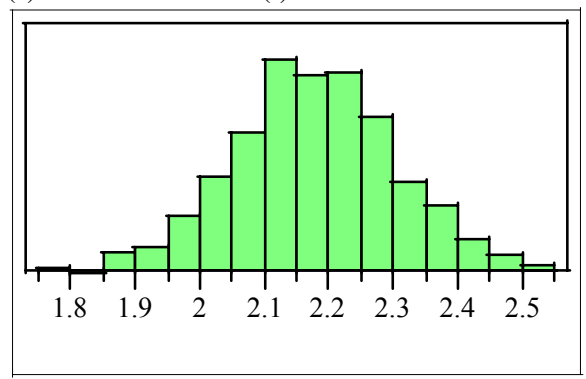

Moments

Mean

2.1782042

Std Dev

upper $95 \%$ Mean

lower $95 \%$ Mea

0.1255322

0.0039697
2.185994

2.185994
2.1704143

1000 


\section{Exhibit A3. Histograms and Other Descriptive Statistics for Inputs to WL Targeting Assuming 1 Sample}

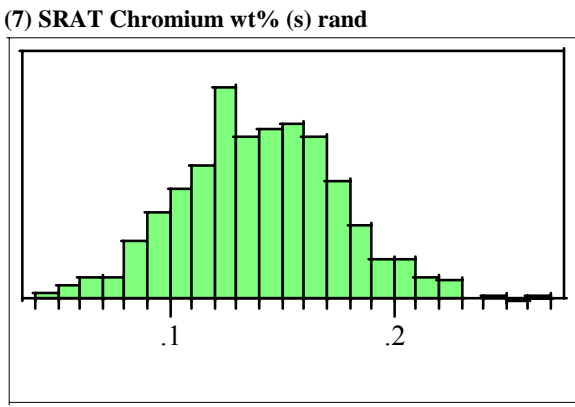

Moments

Mean

Std Dev
Std Err Mean

(1) 95\% Mean

lower $95 \%$ Mean

0.1404361

0.0356621

0.0011277

0.1426491

(7) SRAT Copper wt \% (s) rand

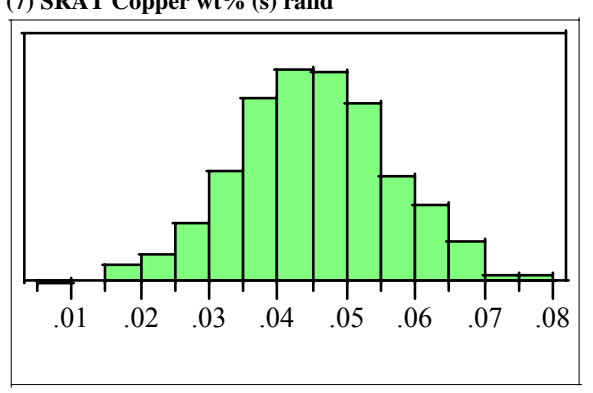

Moments

Mean

Std Dev

lower $95 \%$ Mean

0.0452202

0.0112898

0.000357
0.0459207

0.0445196

1000
(7) SRAT Iron wt\% (s) rand

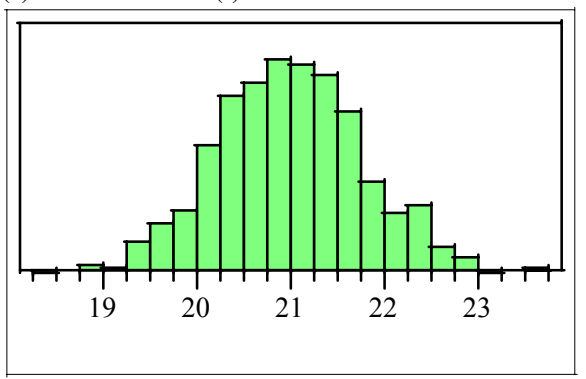

Moments

Mean

Std Err Mean

Std Err Mean

upper $95 \%$ Mean

20.992091

0.771214
0.0243879

0.0243879

21.039948

(7) SRAT Potassium wt\% (s) rand

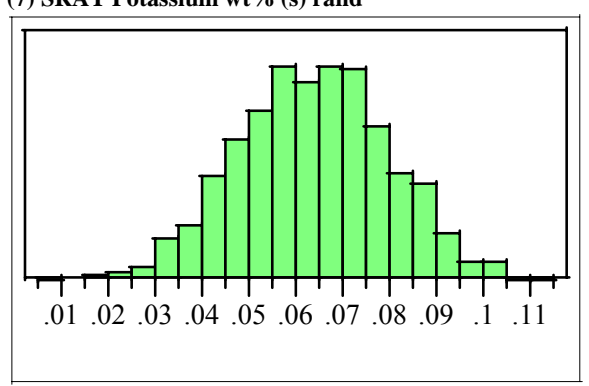

Moments

Mean

Std Err Mean

lower $95 \%$ Mean

0.0004991

0.0628233
(7) SRAT Lithium wt\% (s) rand

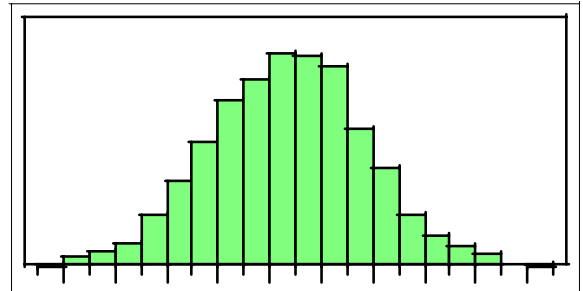

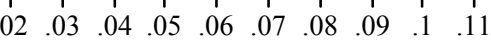

Moments

Mean

Std Err Mean

upper $95 \%$ Mean

lower 95\% Mean

0.06335

0.0004713

0.0642749

(7) SRAT Magnesium wt\% (s) rand

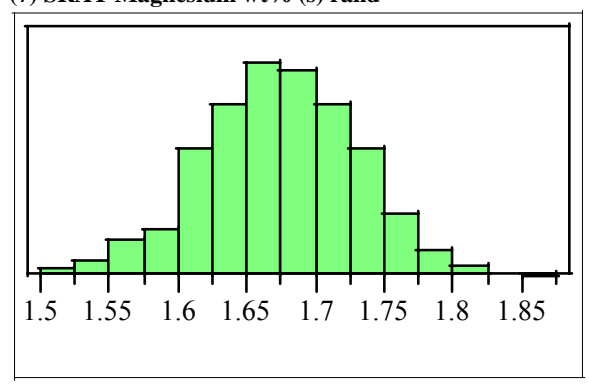

Moments

Mean

Std Err Mean

upper 95\%

lower $95 \%$ Mea

1.6737464

0.0547707
0.001732

0.001732
1.6771452
1.6703476

1000

1000 
Exhibit A3. Histograms and Other Descriptive Statistics for Inputs to WL Targeting Assuming 1 Sample

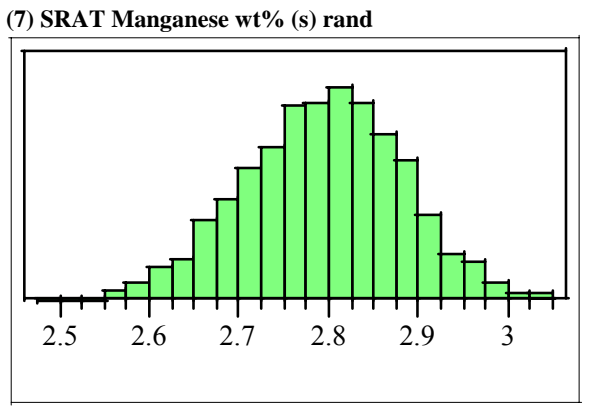

Moments

Mean

Std Dev

Std Err Mean

lower $95 \%$ Mean

2.793822

0.088917

0.0028118
2.7993397

2.7883042

(7) SRAT Sodium wt\% (s) rand

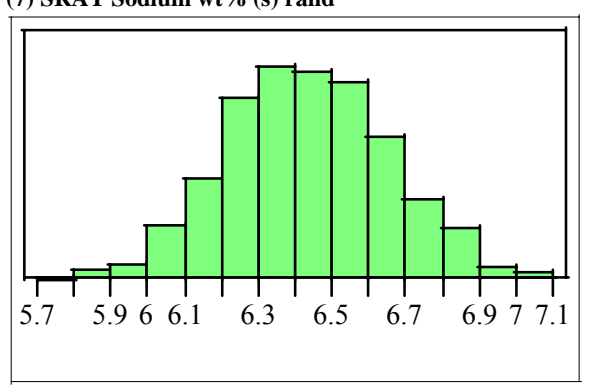

Moments

Mean

Std Dev

lower 95\% Mea

ower 95\% Mean

6.4341759

0.2244619

6.4481049

6.4481049
(7) SRAT Nickel wt $\%$ (s) rand

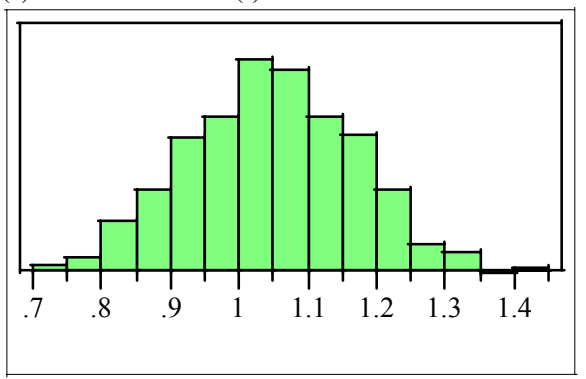

Moments

Mean

Std Dev

ster $95 \%$ Mean

upper $95 \%$ Mean

1.0473038

0.1223274

(7) SRAT Silicon wt\% (s) rand

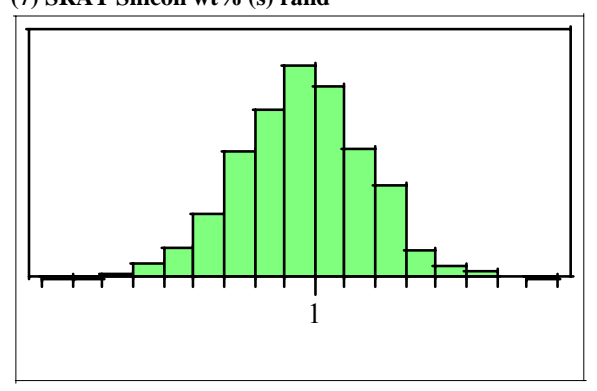

Moments

Mean

Std Err Mean

upper 95\% Mean

upper $95 \%$ Mea

$\mathrm{N}$

1000
(7) SRAT Titanium wt\% (s) rand

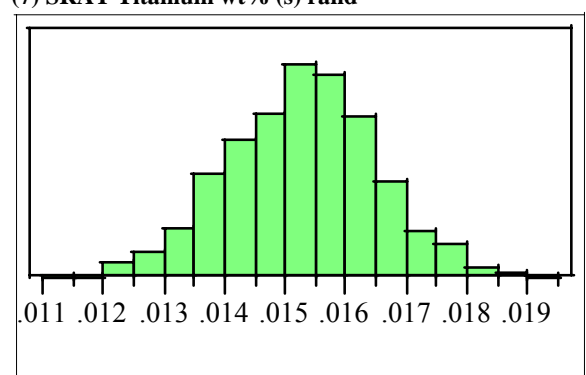

Moments

Mean

0.015275

0.0012272

0.00153512

Std Err Mean

upper 95\% Mean

0.0153512

$\mathrm{N}$

(7) SRAT Uranium wt\% (s) rand

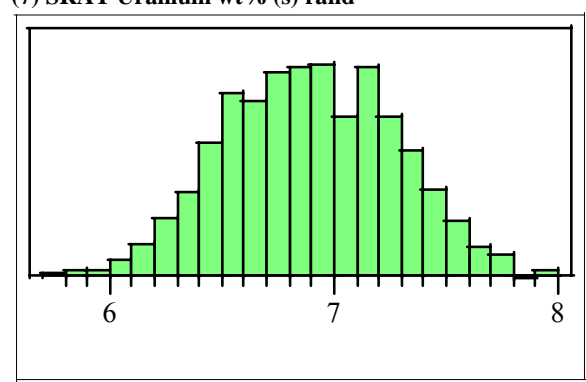

Moments

Mean

Std Err Mean

upper $95 \%$ Mean

lower $95 \%$ Mea

0.0120776
6.9163394

6.9163394

1000 
Exhibit A3. Histograms and Other Descriptive Statistics for Inputs to WL Targeting Assuming 1 Sample

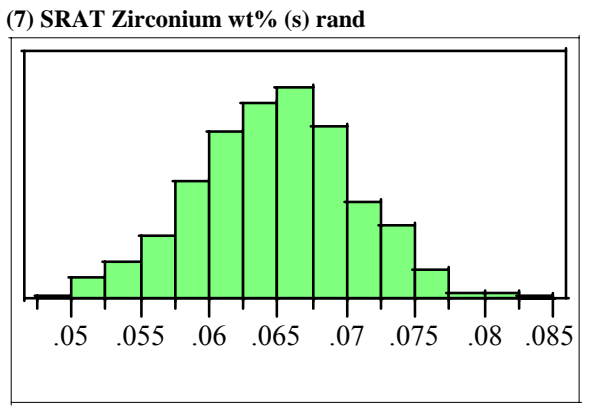

Moments

Mean

Std Err Mea

St

lower $95 \%$ Mean

0.0648119

0.0058987

0.0001865

0.065178

(8) SRAT Volume (gals) rand

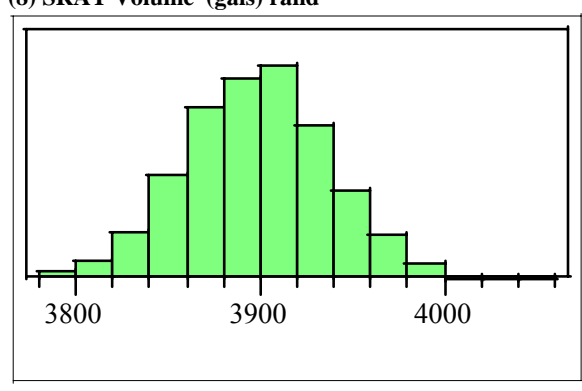

Moments

Mean

Std Dev

lower $95 \%$ Mean

3898.2007

38.986737

1.2328689

3900.62
(9) Frit 320 Aluminum wt \% (v) rand

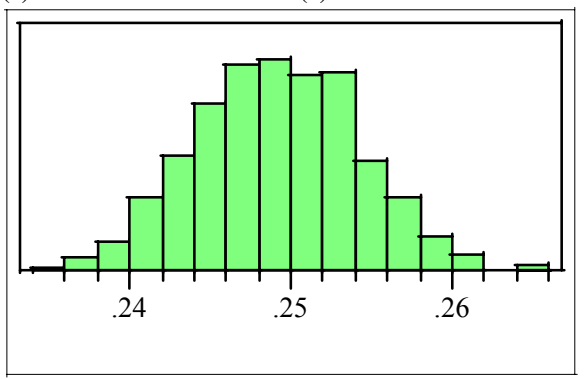

Moments

Mean

Std Err Mean

upper $95 \%$ Mean

upper $95 \%$ Mean

0.2491261

0.0050682

0.2488116

1000

(9) Frit 320 Boron wt\% (v) rand

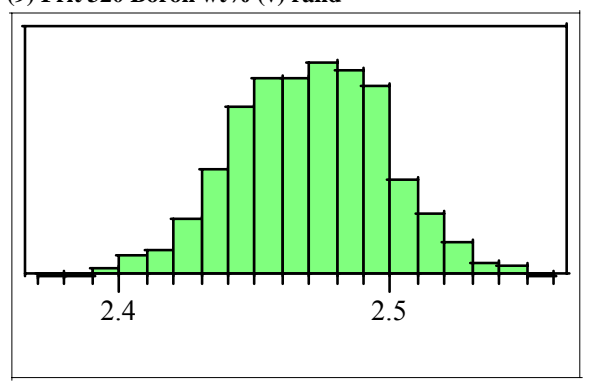

Moments

Mean

Std Dev

upper 95\% Mean

upper $95 \%$ Mea

2.472004
(9) Frit 320 Calcium wt\% (v) rand

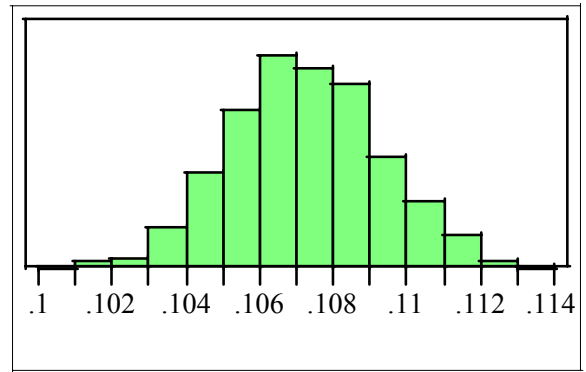

Moments

Mean

Std Err Mean

upper $95 \%$ Mean

0.1072404

0.0000643

0.1073667

0.1073667

(9) Frit 320 Chromium wt \% (v) rand

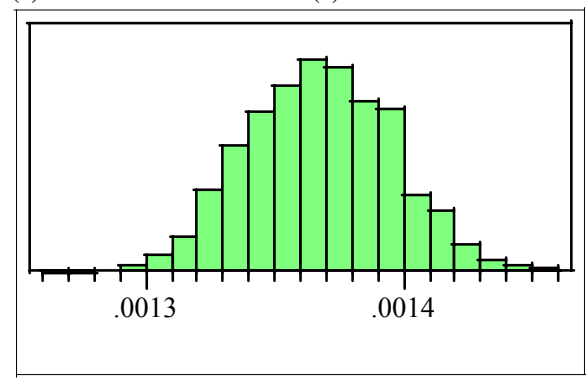

Moments

Mean

0.0013675

Std Dev

upper $95 \%$ Mea

lower $95 \%$ Mea

0.000028

$8.9531 \mathrm{e}-7$

0.0013693

1000

1000 
Exhibit A3. Histograms and Other Descriptive Statistics for Inputs to WL Targeting Assuming 1 Sample

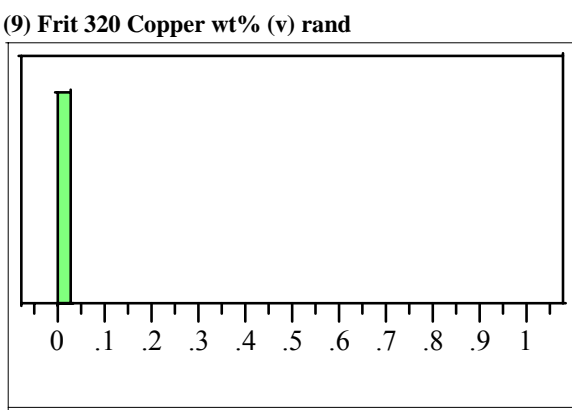

Moments

Mean

Std Err Men

Utd Err Mean

lower $95 \%$ Mean

(9) Frit 320 Iron wt \% (v) rand

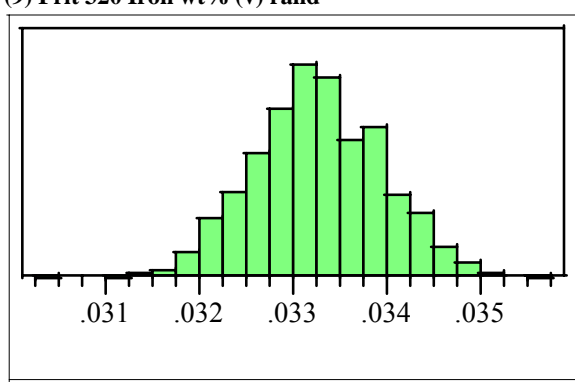

Moments

Mean

Std Dev

lower $95 \%$ Mean

0.0332671

0.0006818

0.0000216

1000
(9) Frit 320 Potassium wt\% (v) rand

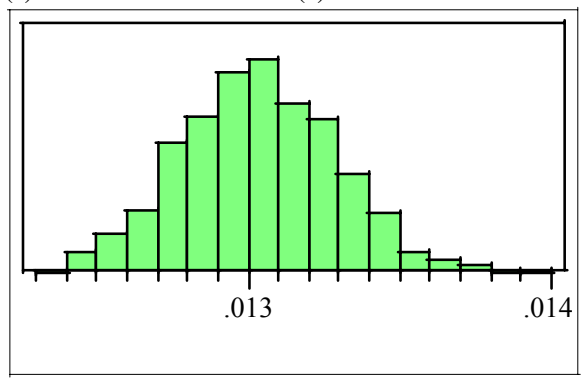

Moments

Mean

Std Dev

St Er $95 \%$ Mean

upper $95 \%$ Mea

0.0130336

0.0002525

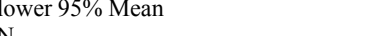

(9) Frit 320 Lithium wt \% (v) rand

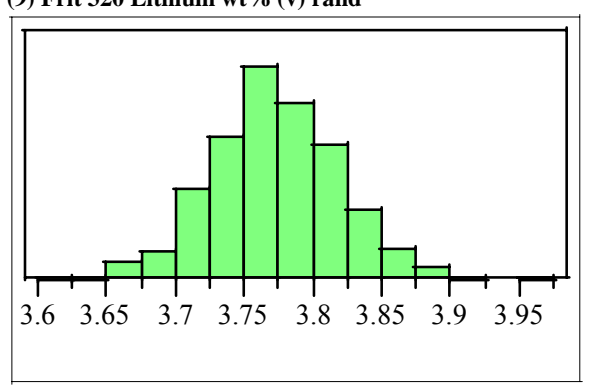

Moments

Mean

Std Err Mean

lower $95 \%$ Mean
(9) Frit 320 Magnesium wt\% (v) rand

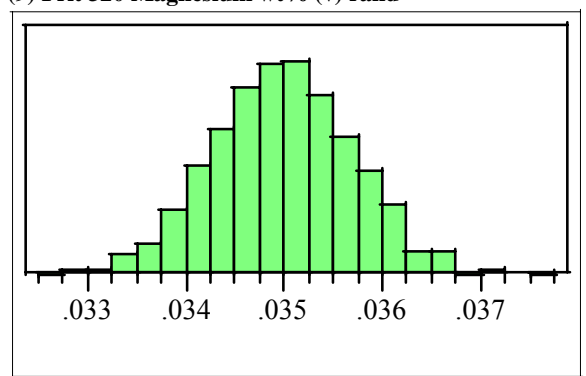

Moments

Mean

Std Err Mean

upper $95 \%$ Mean

lower $95 \%$ Mean

0.0349885

0.0007124

0.000022

0.035032

(9) Frit 320 Manganese wt \% (v) rand

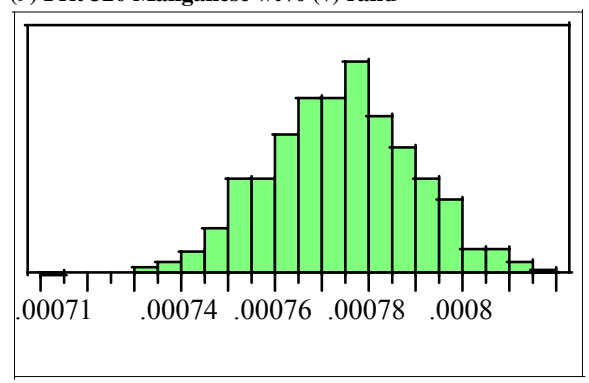

Moments

Mean

Std Err Mean

upper $95 \%$ Mean

lower $95 \%$ Mea

0.00077

0.0000155

$4.9066 \mathrm{e}-7$
0.000775

0.0007731 
Exhibit A3. Histograms and Other Descriptive Statistics for Inputs to WL Targeting Assuming 1 Sample

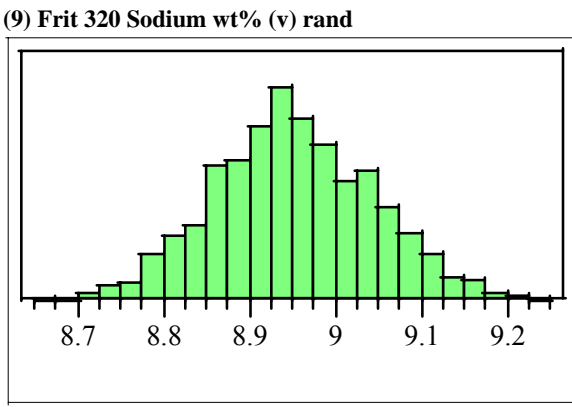

Moments

Mean

Std Dev

Std Err Mean

lower $95 \%$ Mean

8.9511029

0.0930499

0.0029425

8.9568771

(9) Frit 320 Nickel wt\% (v) rand

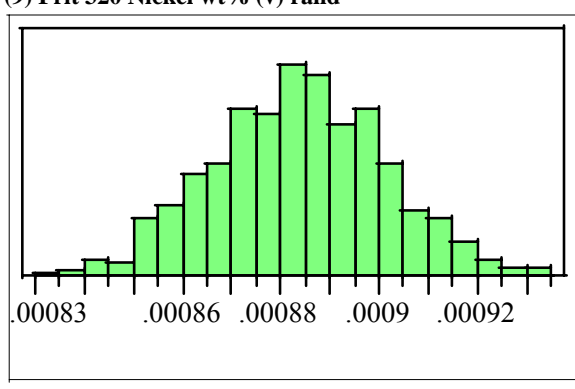

Moments

Mean

Std Dev

upper $95 \%$ Mea

lower $95 \%$ Mean

0.0008838

0.0000176

$5.5725 \mathrm{e}-7$

0.0008849

1000

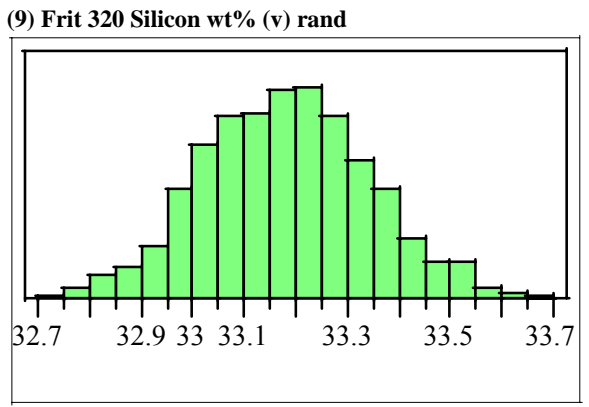

Moments

Mean

Std Err Mean

upper $95 \%$ Mean

upper $95 \%$ Mean

33.179802

0.162745

0.005146

33.189901

$\begin{array}{lr}\mathrm{N} & 1000\end{array}$

(9) Frit 320 Titanium wt \% (v) rand

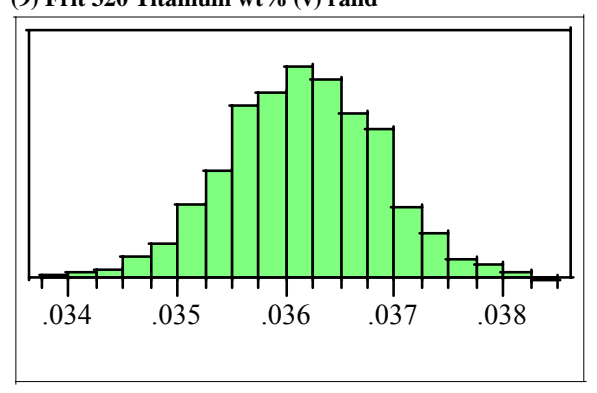

Moments

Mean

Std Dev

Std Err Mean

upper $95 \%$ Mean

lower $95 \%$ Mean

0.0361541

0.0007175

0.0000227

0.0361986

1000
(9) Frit 320 Uranium wt \% (v) rand

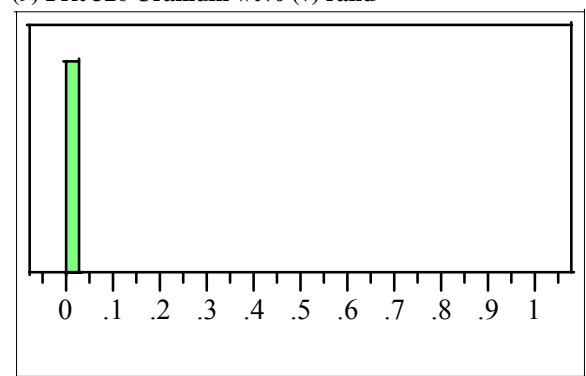

Moments

Mean

Std Err Mean

upper $95 \%$ Mean

lower $95 \%$ Mean

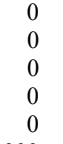

(9) Frit 320 Zirconium wt \% (v) rand

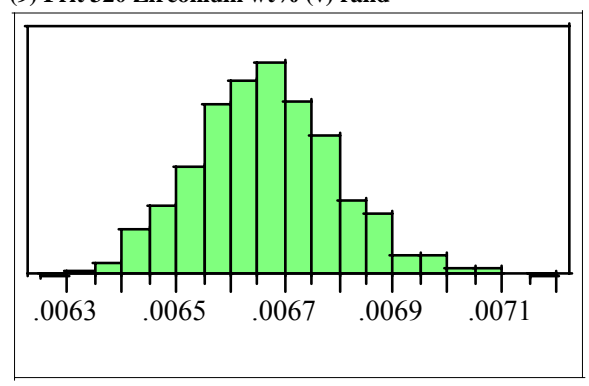

Moments

Mean

Std Err Mean

upper $95 \%$ Mean

lower $95 \%$ Mea

0.0066742

0.006658

1000 


\section{Exhibit A3. Histograms and Other Descriptive Statistics for Inputs to WL Targeting Assuming 1 Sample}

(10) Frit 320 (lbs) rand

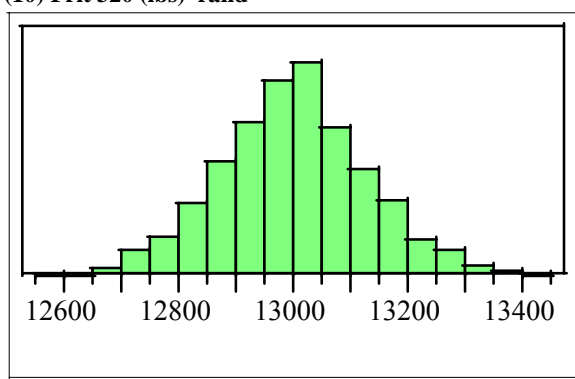

Moments

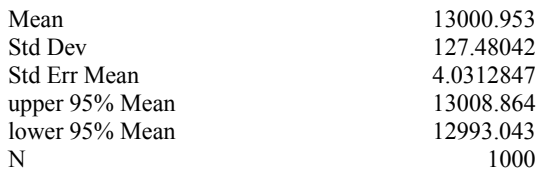

lower 95\% Mean 12993.043

(11) Frit lbs from 5 Can Decon rand

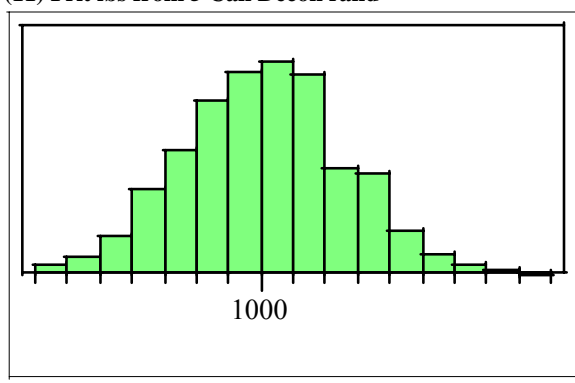

Moments

Mean

1000.6385

1000.6385
24.744295

0.7824833

Std Err Mean $\quad 0.7824833$

upper $95 \%$ Mean $\quad 1002.174$

$\begin{array}{lr}\text { lower 95\% Mean } & 1000 \\ \text { N }\end{array}$ 
Exhibit A4. Overview of WL Uncertainties with Analytical Uncertainties Assuming Nominal Number of Samples

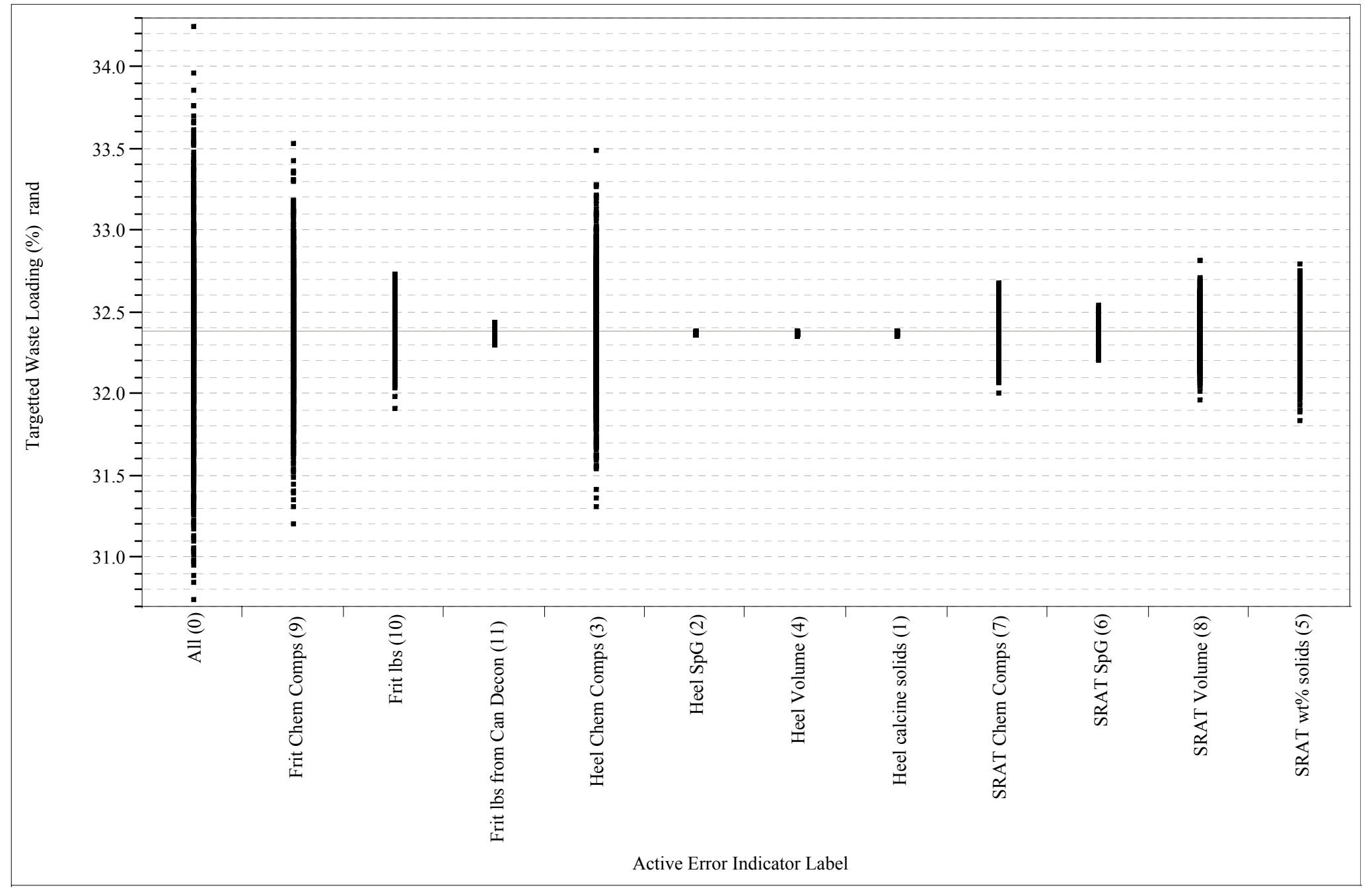


Exhibit A5. Histograms and Other Descriptive Statistics for WLs with Analytical Uncertainties Assuming Nominal Number of Samples

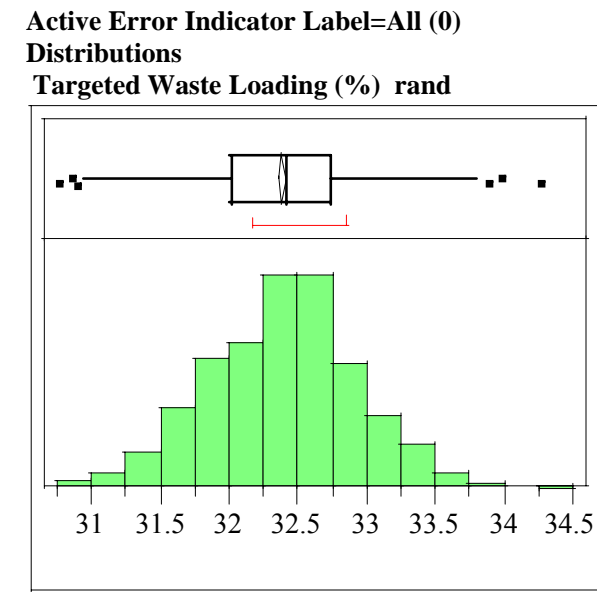

Quantiles

$\begin{array}{llr}100.0 \% & \text { maximum } & 34.261 \\ 99.5 \% & & 33.775 \\ 97.5 \% & & 33.426 \\ 90.0 \% & & 33.048 \\ 75.0 \% & \text { quartile } & 32.734 \\ 50.0 \% & \text { median } & 32.415 \\ 25.0 \% & \text { quartile } & 32.019 \\ 10.0 \% & & 31.671 \\ 2.5 \% & & 31.333 \\ 0.5 \% & & 30.993 \\ 0.0 \% & \text { minimum } & 30.756 \\ \text { Moments } & & \\ & & \\ \text { Mean } & & 32.387782 \\ \text { Std Dev } & & 0.5364729 \\ \text { Std Err Mean } & 0.0169648 \\ \text { upper 95\% Mean } & 32.421073 \\ \text { lower 95\% Mean } & 32.354492 \\ & & 1000\end{array}$

Active Error Indicator Label=Frit Chem Comps (9) Distributions

Targeted Waste Loading (\%) rand

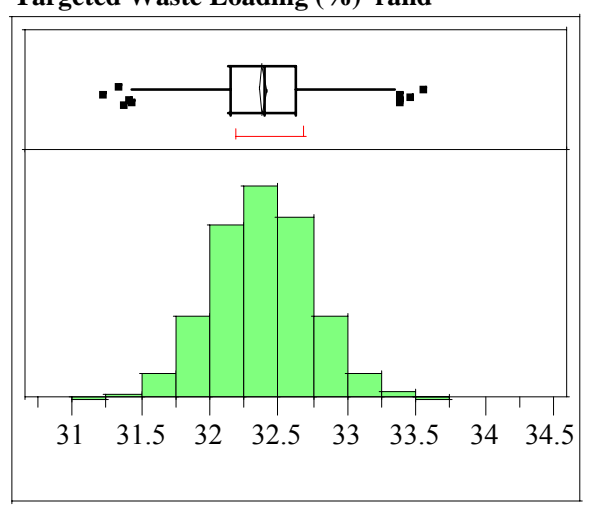

Quantiles

$100.0 \%$ maximum

$99.5 \%$

$97.5 \%$

$90.0 \%$

$75.0 \%$

$50.0 \%$

$25.0 \%$

quartile

median

$10.0 \%$

$2.5 \%$

$0.5 \%$

Moments

minimum

Mean

Std Dev

Std Err Mean

upper $95 \%$ Mean

lower $95 \%$ Mean

$\mathrm{N}$

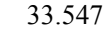$$
33.547
$$$$
33.366
$$$$
33.092
$$$$
32.834
$$$$
32.632
$$$$
32.396
$$$$
32.149
$$$$
31.930
$$$$
\begin{aligned}
& 31.930 \\
& 31.685 \\
& 31.421
\end{aligned}
$$$$
31.685
$$$$
\begin{aligned}
& 31.421 \\
& 31.220
\end{aligned}
$$

32.386018

0.3550432

0.0112275

32.40805
32.363986

1000
Active Error Indicator Label=Frit lbs (10) Distributions

Targeted Waste Loading (\%) rand

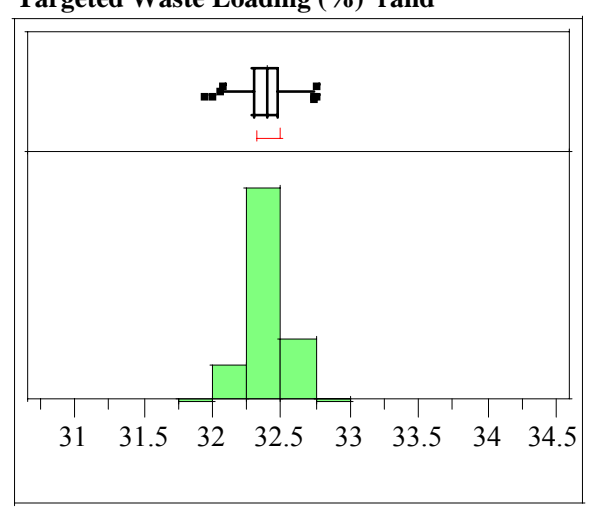

Quantiles

$100.0 \%$ maximum 32.752

$99.5 \% \quad 32.719$

$97.5 \% \quad 32.635$

$90.0 \% \quad 32.551$

$75.0 \%$ quartile 32.476

$50.0 \%$ median 32.398

$25.0 \%$ quartile 32.312

$10.0 \%$

$2.5 \%$

$\begin{array}{lll}0.0 \% & & \\ 0.0 \% & 31.931\end{array}$

Moments

Mean

0.1224269

upper $95 \%$ Mean

lower $95 \%$ Mean $\quad 32.388485$ 
Exhibit A5. Histograms and Other Descriptive Statistics for WLs with Analytical Uncertainties Assuming Nominal Number of Samples

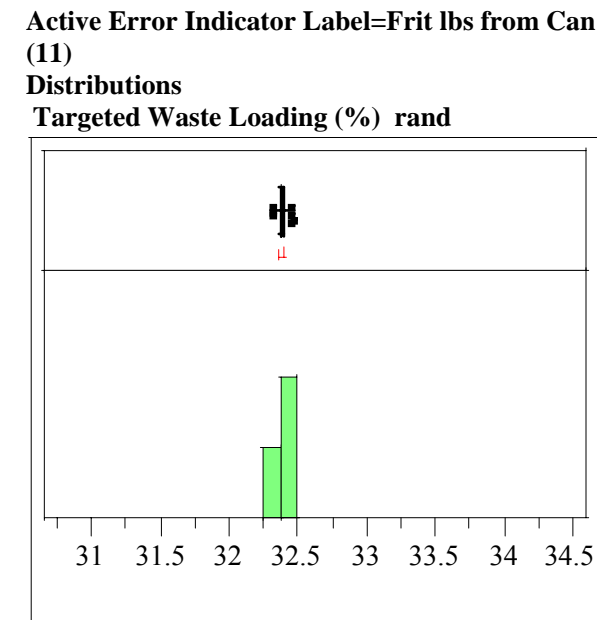

Quantiles

$\begin{array}{llr}100.0 \% & \text { maximum } & 32.457 \\ 99.5 \% & & 32.450 \\ 97.5 \% & & 32.434 \\ 90.0 \% & & 32.418 \\ 75.0 \% & \text { quartile } & 32.402 \\ 50.0 \% & \text { median } & 32.386 \\ 25.0 \% & \text { quartile } & 32.370 \\ 10.0 \% & & 32.355 \\ 2.5 \% & & 32.341 \\ 0.5 \% & & 32.327 \\ 0.0 \% & \text { minimum } & 32.314 \\ \text { Moments } & & \\ & & \\ \text { Mean } & & 32.385901 \\ \text { Std Dev } & & 0.0239868 \\ \text { Std Err Mean } & 0.0007585 \\ \text { upper 95\% Mean } & 32.38739 \\ \text { lower 95\% Mean } & 32.384413 \\ \text { N } & 1000\end{array}$

Active Error Indicator Label=Heel Chem Comps (3) Distributions

Targeted Waste Loading (\%) rand

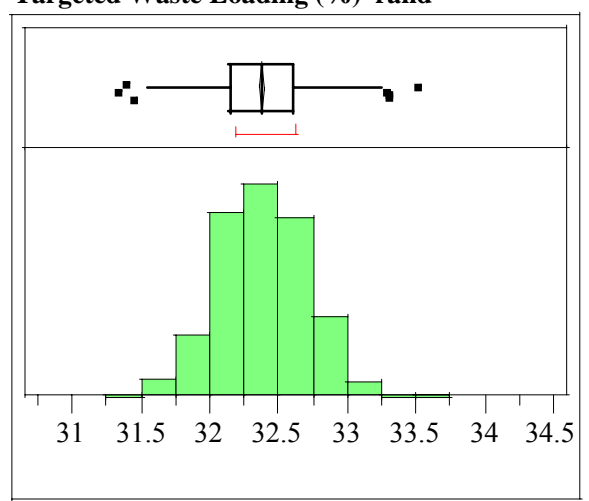

Quantiles

$\begin{array}{llr}100.0 \% & \text { maximum } & 33.509 \\ 99.5 \% & & 33.236 \\ 97.5 \% & & 32.984 \\ 90.0 \% & & 32.800 \\ 75.0 \% & \text { quartile } & 32.601 \\ 50.0 \% & \text { median } & 32.383 \\ 25.0 \% & \text { quartile } & 32.158 \\ 10.0 \% & & 31.984 \\ 2.5 \% & & 31.746 \\ 0.5 \% & & 31.565 \\ 0.0 \% & \text { minimum } & 31.331 \\ \text { Moments } & & \\ & & \\ \text { Mean } & & 32.382192 \\ \text { Std Dev } & & 0.318699 \\ \text { Std Err Mean } & 0.0100781 \\ \text { upper 95\% Mean } & 32.401969 \\ \text { lower 95\% Mean } & 32.362416 \\ \text { N } & & 1000\end{array}$

Active Error Indicator Label=Heel SpG (2) Distributions

Targeted Waste Loading (\%) rand

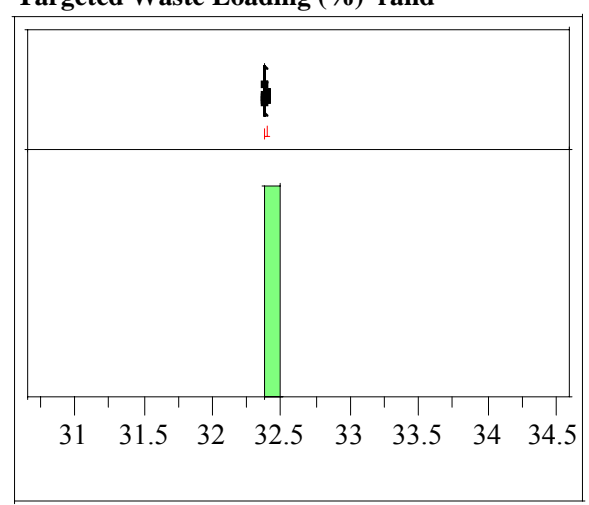

Quantiles

$100.0 \% \quad$ maximum $\quad 32.396$

$99.5 \% \quad 32.395$

$97.5 \% \quad 32.392$

$90.0 \%-32.390$

$75.0 \% \quad$ quartile $\quad 32.388$

$50.0 \%$ median 32.386

$25.0 \%$ quartile 32.384

$2.5 \%$

$2.5 \%-5 \%-2-0$

$\begin{array}{ll}0.5 \% & \\ 0.0 \% & \text { minimum }\end{array}$

Moments

Mean

0.0030879

lower $95 \%$ Mean $\quad 32.385837$ 
WSRC-TR-2004-00508

Revision 0

Exhibit A5. Histograms and Other Descriptive Statistics for WLs with Analytical Uncertainties Assuming Nominal Number of Samples

Active Error Indicator Label=Heel Volume (4) Distributions

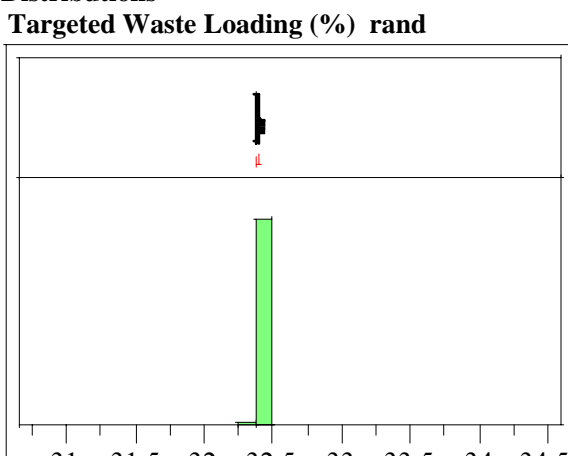

$\begin{array}{llllllll}31 & 31.5 & 32 & 32.5 & 33 & 33.5 & 34 & 34.5\end{array}$

Quantiles

$100.0 \%$ maximum 32.404

$97.5 \%$

$75.0 \%$ quartile

$50.0 \% \quad$ median

$25.0 \%$ quartile

$10.0 \%$

$2.5 \%$

$0.5 \%$

minimum

32.402

32.396

32.390

32.386

32.382

32.379

32.375

32.372

Moments

32.371

Mean

Std Dev

Std Err Mean

upper $95 \%$ Mean

lower $95 \%$ Mean

$\mathrm{N}$

32.386078

0.0056239

0.0001778

32.386427

32.385729

1000
Active Error Indicator Label=Heel calcine solids (1) Distributions

Targeted Waste Loading (\%) rand

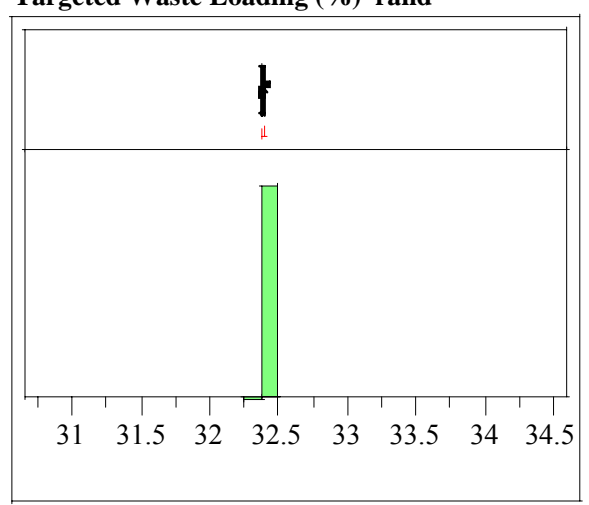

Quantiles

$100.0 \% \quad$ maximum $\quad 32.400$

$99.5 \% \quad 32.396$

$99.5 \%$

$75.0 \%$

quartile

median

$25.0 \%$ quartile

$10.0 \%$

$2.5 \%$

$0.5 \%$

Moments

minimum

Mean

Std Dev

Std Err Mean

upper $95 \%$ Mean

lower $95 \%$ Mean

$\mathrm{N}$

32.392

32.389

32.386

32.383

32.380

32.378

32.371

2.386076

0.004302

0.000136

32.386343

32.385809

1000
Active Error Indicator Label=SRAT Chem Comps (7) Distributions

Targeted Waste Loading (\%) rand

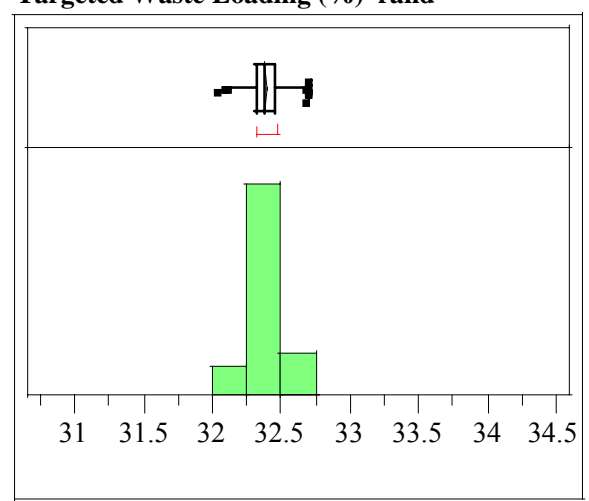

Quantiles

$100.0 \%$ maximum 32.697

$99.5 \%$

$97.5 \%$

$90.0 \%-32.533$

$\begin{array}{ll}75.0 \% & \text { quartile } \\ 50.0 \% & 32.456\end{array}$

$50.0 \%$ median 32.379

$25.0 \%$ quartile 32.316

32.244

$0.5 \%$

$0.0 \% \quad$ minimum $\quad 32.022$

Moments

Mean

Std Dev

Std Err Mean

upper $95 \%$ Mean

lower $95 \%$ Mean

32.383876

0.1083588

32.39060

32.377152 
WSRC-TR-2004-00508

Revision 0

Exhibit A5. Histograms and Other Descriptive Statistics for WLs with Analytical Uncertainties Assuming Nominal Number of Samples

Active Error Indicator Label=SRAT SpG (6) Distributions

Targeted Waste Loading (\%) rand

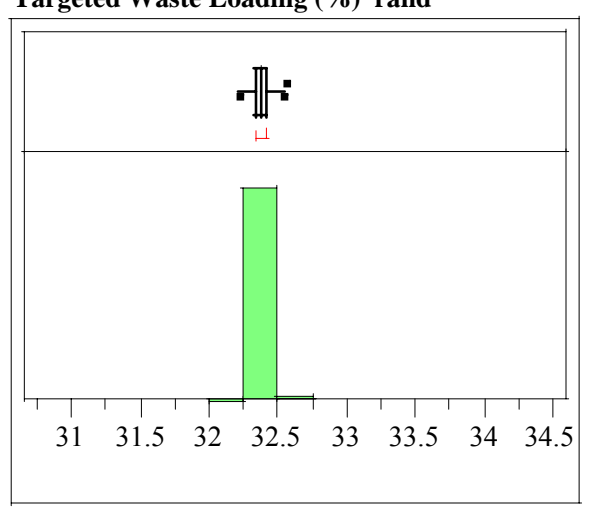

Quantiles

$100.0 \%$ maximum 32.562

$97.5 \%$

$90.0 \%$

$75.0 \%$ quartile

$50.0 \% \quad$ median

$25.0 \%$ quartile

$10.0 \%$

$2.5 \%$

$0.5 \%$

minimum

32.487

32.451

32.423

32.383

32.347

32.312

32.280

32.241

Moments

32.225

Mean

Std Dev

Std Err Mean

upper $95 \%$ Mean

lower $95 \%$ Mean

32.38419

0.0544885

32.387571

32.387571

1000
Active Error Indicator Label=SRAT Volume (8) Distributions

Targeted Waste Loading (\%) rand

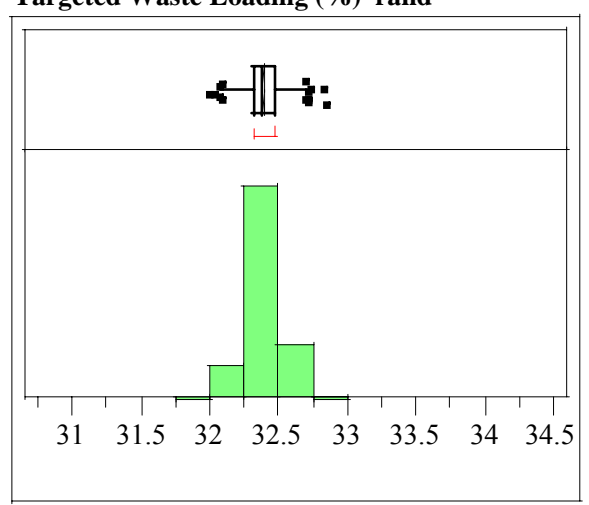

Quantiles

$100.0 \% \quad$ maximum $\quad 32.836$

$99.5 \% \quad 32.710$

32.710

$97.5 \%$

$\begin{array}{lll}75.0 \% & \text { quartile } & 32.553 \\ 50.0 \% & 32.466\end{array}$

$50.0 \%$ median

$25.0 \%$ quartile

32.243

$2.5 \% \quad 32.159$

$0.5 \%$

$0.0 \% \quad$ minimum 31.983

Moments

32.392537

Std Dev

Std Err Mean

upper $95 \%$ Mean

lower $95 \%$ Mean

$\mathrm{N}$

0.1199607

0.0037935

32.39998

385093
Active Error Indicator Label=SRAT wt \% solids (5) Distributions

Targeted Waste Loading (\%) rand

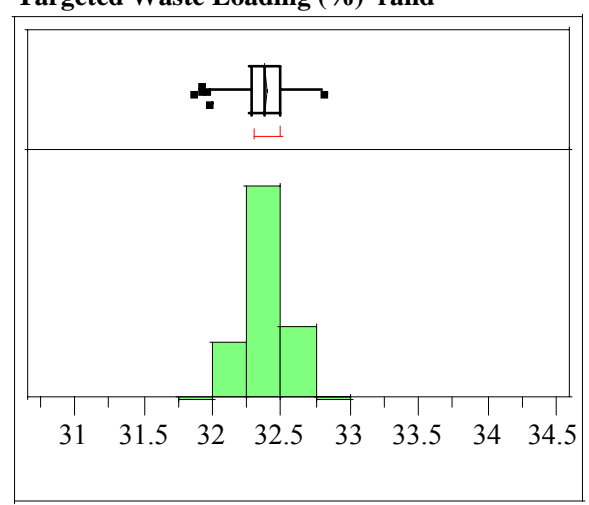

Quantiles

$100.0 \%$ maximum 32.809

$99.5 \%$

$97.5 \%-32.682$

$90.0 \% \quad 32.575$

$75.0 \%$ quartile 32.486

$50.0 \%$ median 32.387

$25.0 \%$ quartile 32.29

32.195

$2.5 \% \quad 32.098$

$\begin{array}{ll}0.5 \% & \\ 0.0 \% & \text { minimum }\end{array}$

Moments

Mean

0.1460066

upper $95 \%$ Mean

lower 95\% Mean $\quad 32.377339$ 
Exhibit A6. Histograms and Other Descriptive Statistics for Inputs to WL Targeting Assuming Nominal Number of Samples

Error Indicator \#=0

(1) SME Heel Calcined wt $\%$ solids wt $\%$ (s) rand

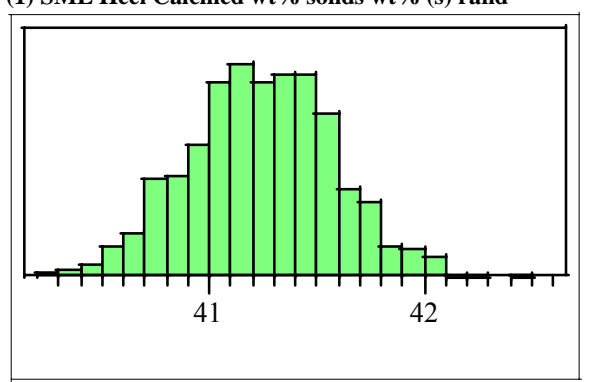

Moments

Mean

Std Dev

upper 95\% Mean

lower $95 \%$ Mean

41.241983

0.3356037

41.262809

41.262809

(2) SME Heel Specific gravity spgr rand

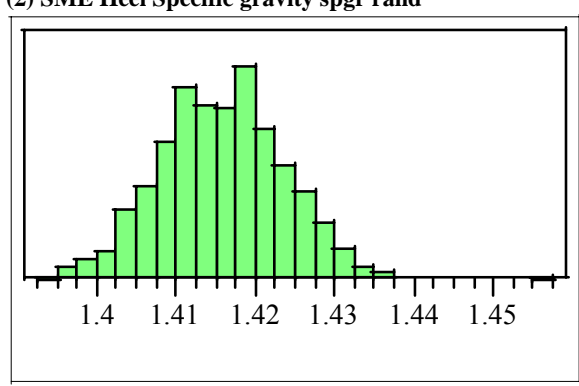

Moments

Mean

Std Dev

upper $95 \%$ Mea

lower $95 \%$ Mean

1.4157401

0.0002463

0.0002463

1.4162234

1000
(3) SME Heel Aluminum wt \% (v) rand

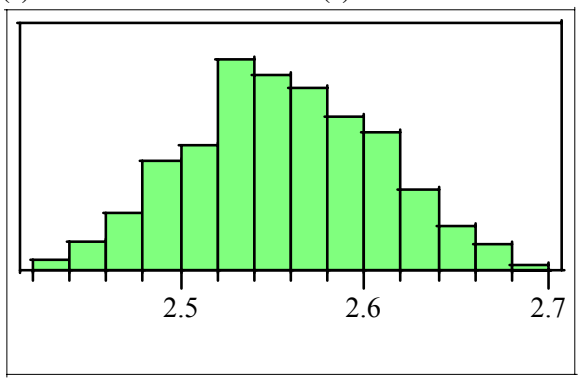

Moments

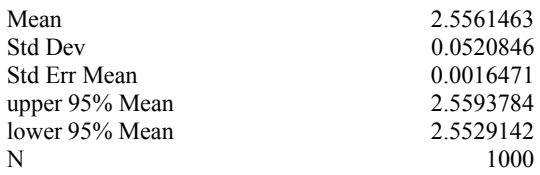

(3) SME Heel Boron wt \% (v) rand

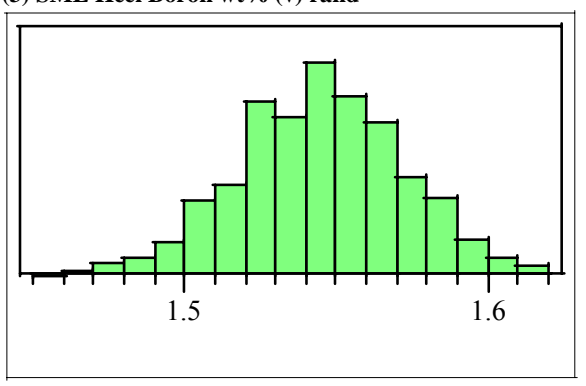

Moments

Mean

1.5447169

Std Dev

upper $95 \%$ Mean

lower $95 \%$ Mea

$\mathrm{N}$
(3) SME Heel Calcium wt\% (v) rand

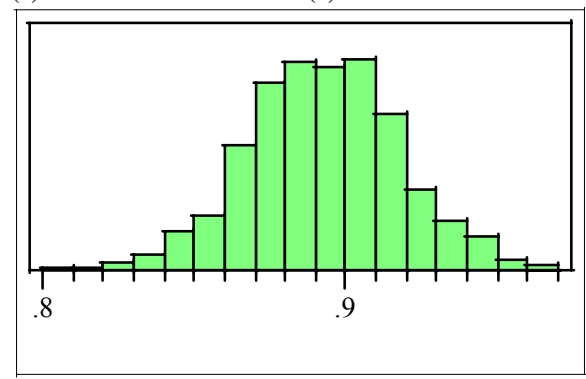

Moments

Mean

Std Err Mean

upper $95 \%$ Mean

lower $95 \%$ Mean

0.8927972

0.025545

0.0008078

0.8943825

(3) SME Heel Chromium wt \% (v) rand

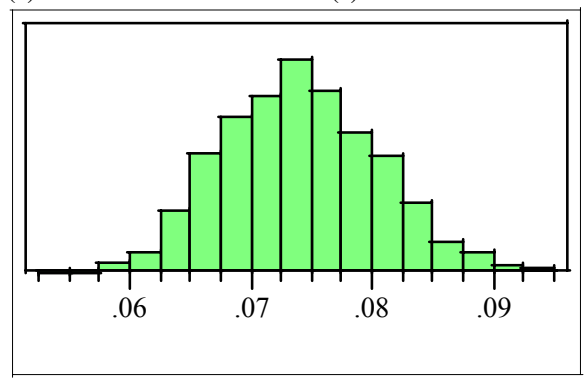

Moments

Mean

Std Err Mean

upper $95 \%$ Mea

lower $95 \%$ Mea

0.074057

0.0063134

0.0744487

0.0736652

1000 
Exhibit A6. Histograms and Other Descriptive Statistics for Inputs to WL Targeting Assuming Nominal Number of Samples

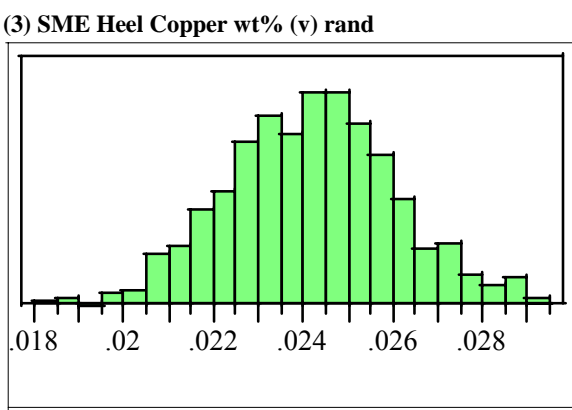

Moments

Mean

Std Dev

Std Err Mean

lower $95 \%$ Mean

0.0241915

0.0018775

0.0000594

0.024308

(3) SME Heel Iron wt\% (v) rand

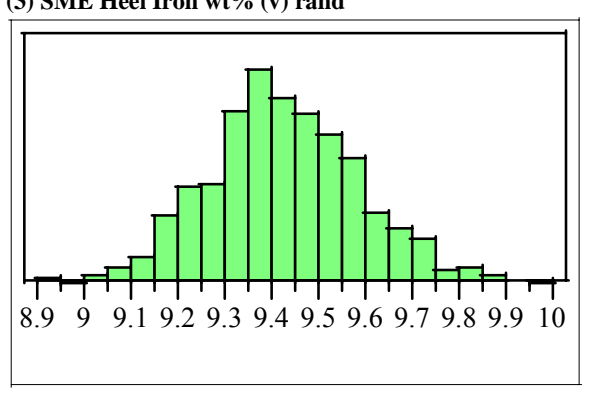

Moments

Mean

Std Dev

lower $95 \%$ Mea

ower 95\% Mean

9.424205

0.1602945

0.005069

9.434152

1000
(3) SME Heel Potassium wt\% (v) rand

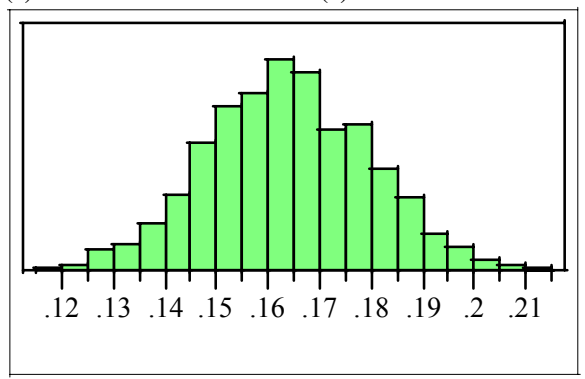

Moments

Mean

Std Dev
Std Err Mean

ster $95 \%$ Mean

upper $95 \%$ Mean

0.1639478

0.0162079

0.0005125

0.1649536

1000

(3) SME Heel Lithium wt\% (v) rand

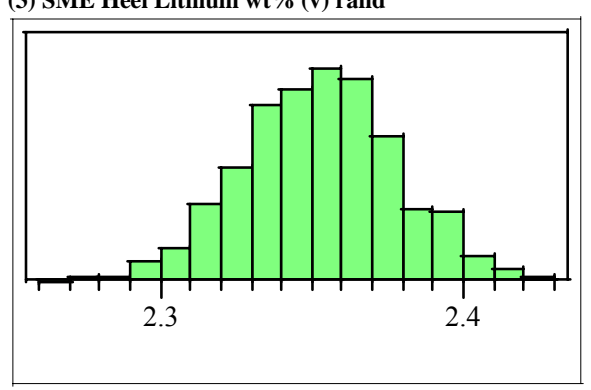

Moments

Mean

Std Dev

upper 95\% Mean

upper $95 \%$ Mea

$\mathrm{N}$

2.354336

1000
(3) SME Heel Magnesium wt\% (v) rand

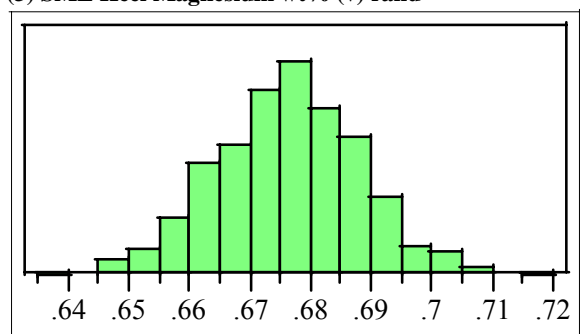

Moments

Mean

Std Err Mean

upper $95 \%$ Mean

0.0116049

0.6768766

0.6768766

(3) SME Heel Manganese wt \% (v) rand

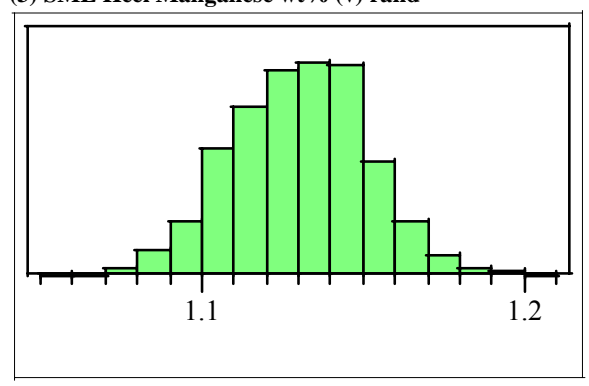

Moments

Mean

Std Err Me

upper 95\% Mean

lower $95 \%$ Mea

1.1299694

0.0210358

0.0006652 
Exhibit A6. Histograms and Other Descriptive Statistics for Inputs to WL Targeting Assuming Nominal Number of Samples

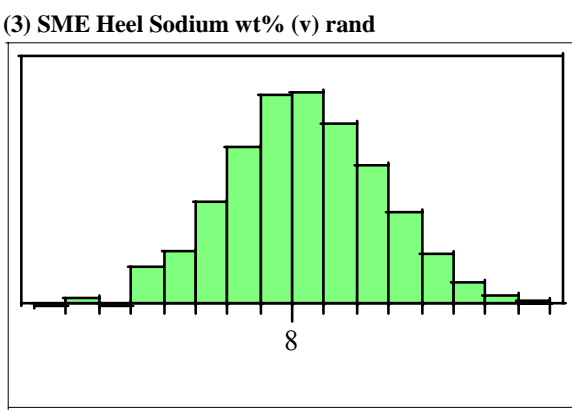

Moments

Mean

Std Dev

Std Err Mean

lower $95 \%$ Mean

8.0362043

0.2415546

0.0076386

8.0511939

(3) SME Heel Nickel wt\% (v) rand

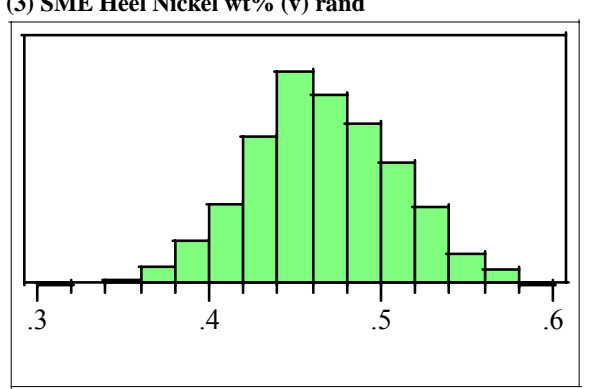

Moments

Mean

Std Dev

S

lower $95 \%$ Mea

ower $95 \%$ Mean

0.4666291

0.0423369

0.0013388

0.4692563

1000
(3) SME Heel Silicon wt \% (v) rand

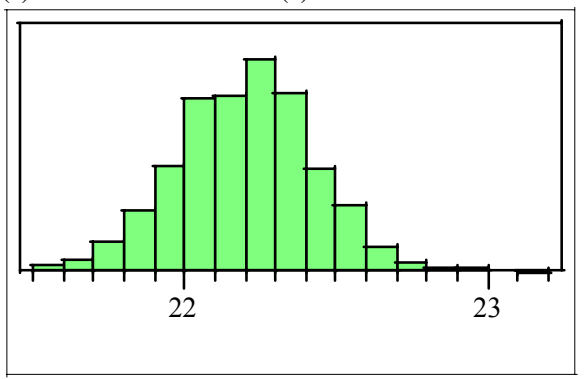

Moments

Mean

Std Err Mean

upper $95 \%$ Mean

upper $95 \%$ Mean

0.2282597

0.0072182

22.21603

N

(3) SME Heel Titanium wt\% (v) rand

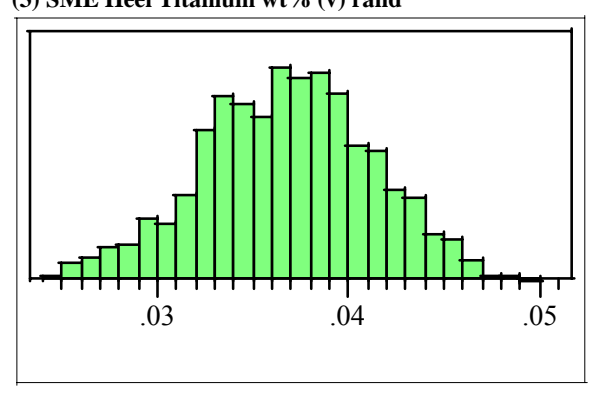

Moments

Mean

Std Dev

upper 95\% Mean

upper $95 \%$ Mea

$\mathrm{N}$
0.0367647

0.0044552

0.0001409

0.0370412

1000
(3) SME Heel Uranium wt\% (v) rand

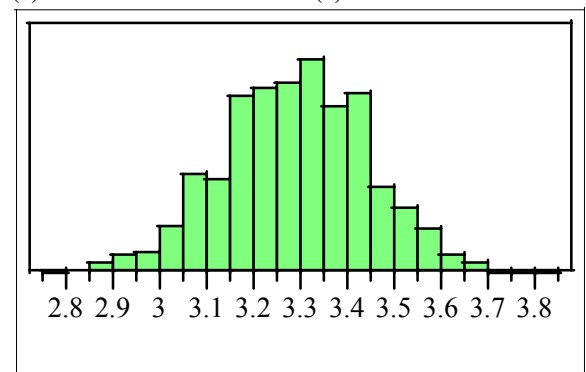

Moments

Mean

Std Err Mean

upper $95 \%$ Mean

lower $95 \%$ Mean

3.2888142

0.1544408

0.0048838

3.298398

(3) SME Heel Zirconium wt\% (v) rand

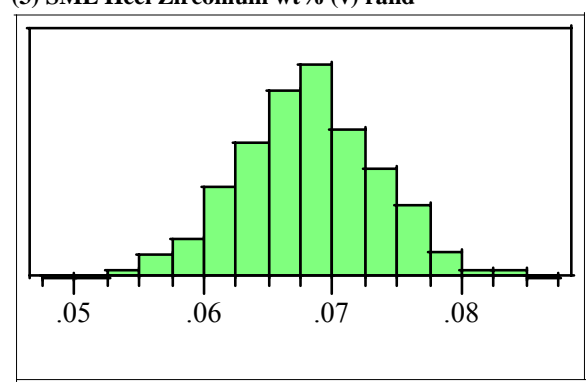

Moments

Mean

Std Err Mean

upper $95 \%$ Mean

lower $95 \%$ Mea 
Exhibit A6. Histograms and Other Descriptive Statistics for Inputs to WL Targeting Assuming Nominal Number of Samples

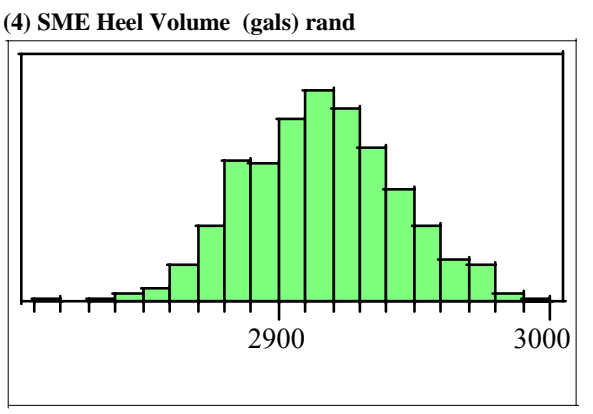

Moments

Mean

Std Dev

Std Err Mean

lower $95 \%$ Mean

2915.5008

28.622453

0.9051214

2917.277

1000

(5) SRAT Total wt \% solids wt $\%$ (s) rand

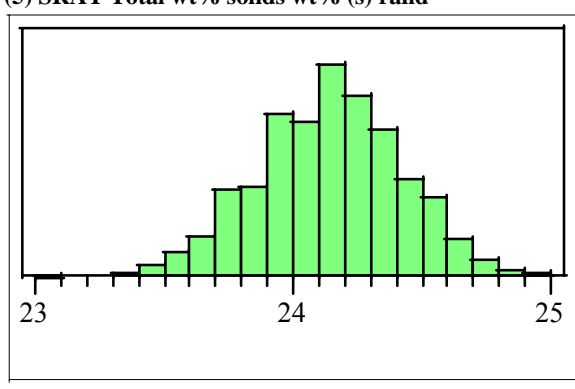

Moments

Mean

Std Dev

lower $95 \%$ Mea

ower $95 \%$ Mean

24.139466

0.2800148

0.0088548

24.156842

1000
(6) SRAT Specific gravity spgr rand

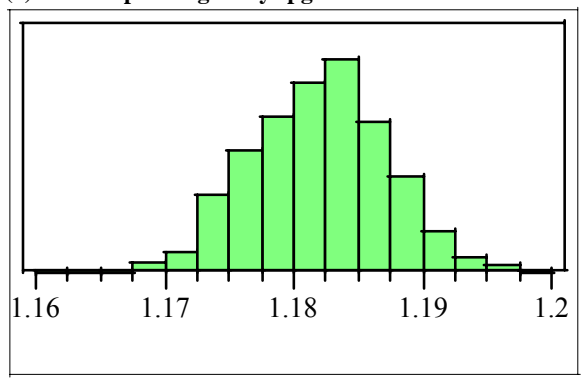

Moments

Mean

Std Err Mean

upper $95 \%$ Mean

upper $95 \%$ Mean

(7) SRAT Aluminum wt\% (s) rand

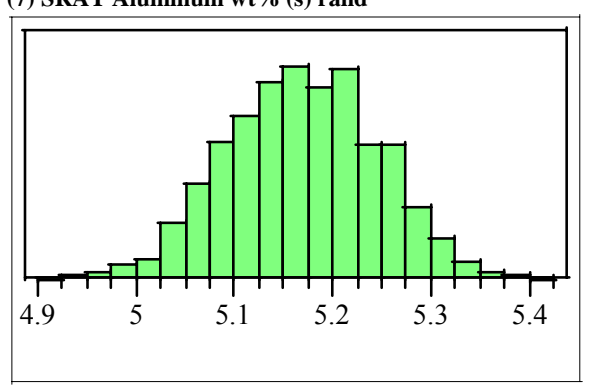

Moments

Mean

Std Err Mean

upper $95 \%$ Mean

upper $95 \%$ Mea

$\mathrm{N}$

0.0770177

0.0024355
5.173551
5.1639924

1000
(7) SRAT Boron wt \% (s) rand

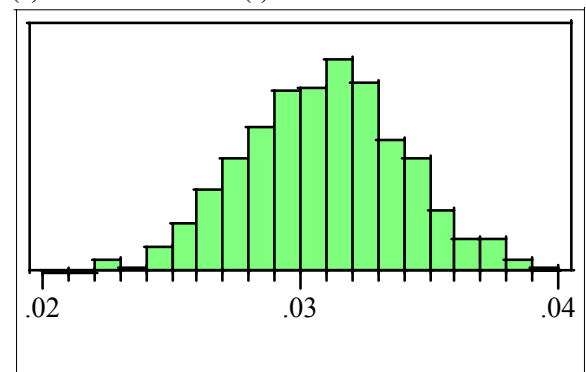

Moments

Mean

Std Err Mean

upper 95\% Mean

0.0031258

0.0000988

0.0310661

0.000168

1.1822864
1.1816266

(7) SRAT Calcium wt \% (s) rand

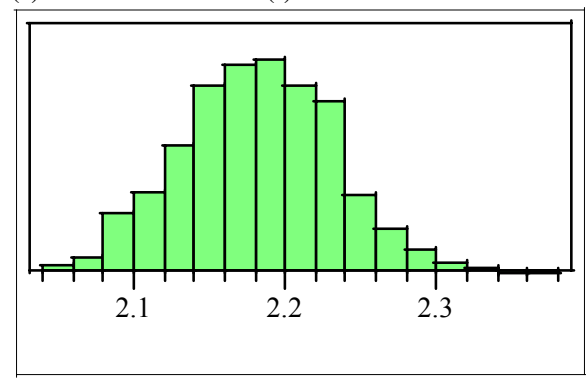

Moments

Mean

Std Dev

upper 95\% Mean

lower $95 \%$ Mea

2.1818946

0.0500017

2.1849975

2.1849975

1000 
Exhibit A6. Histograms and Other Descriptive Statistics for Inputs to WL Targeting Assuming Nominal Number of Samples

(7) SRAT Chromium wt\% (s) rand

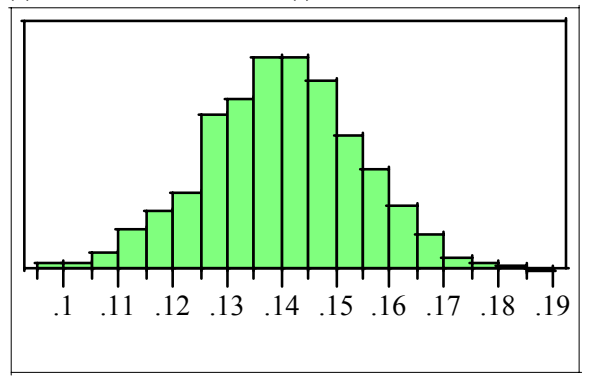

Moments

Mean

Std Err Mean

upper $95 \%$ Mean

lower $95 \%$ Mean

0.1399624

0.0144149

0.0004558

0.1408569

(7) SRAT Copper wt $\%$ (s) rand

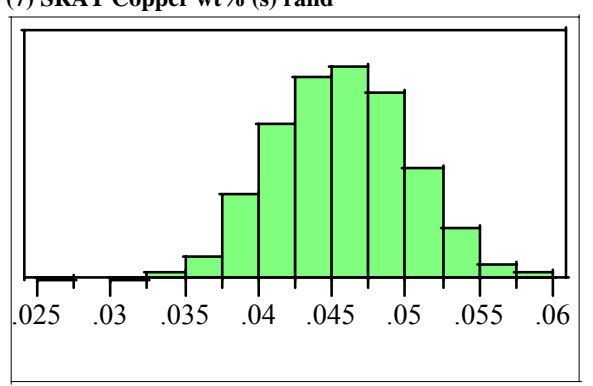

Moments

Mean

Std Dev

upper $95 \%$ Mea

upper $95 \%$ Mea

lower $95 \%$ Mean

0.0456142

0.004589

0.0001451

0.0453294

1000

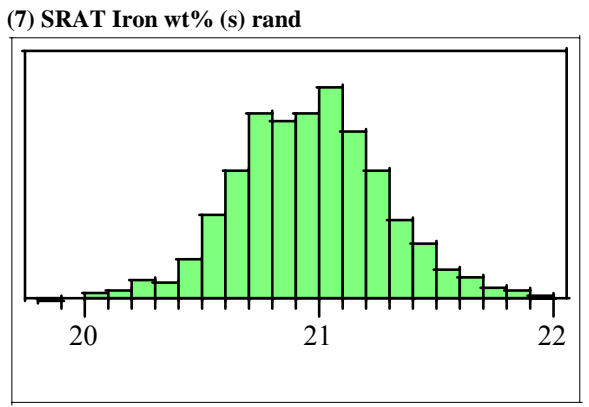

Moments

Mean

Std Dev
Std Err Mean

upper $95 \%$ Mean

upper $95 \%$ Mean

(7) SRAT Potassium wt\% (s) rand

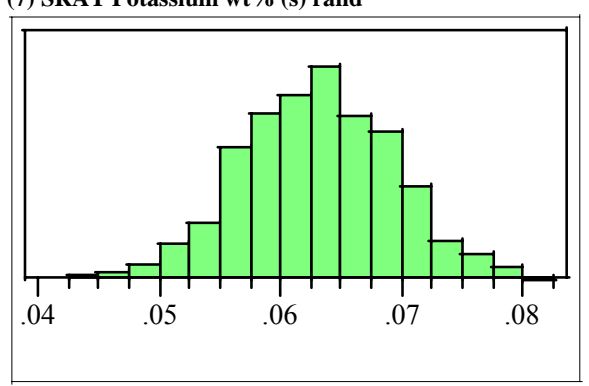

Moments

Mean

Std Dev

Std Err Mean

upper $95 \%$ Mean

lower $95 \%$ Mean

0.3166484

0.0013

20.98061

1000

0.0062546

0.0001978

1000
(7) SRAT Lithium wt \% (s) rand

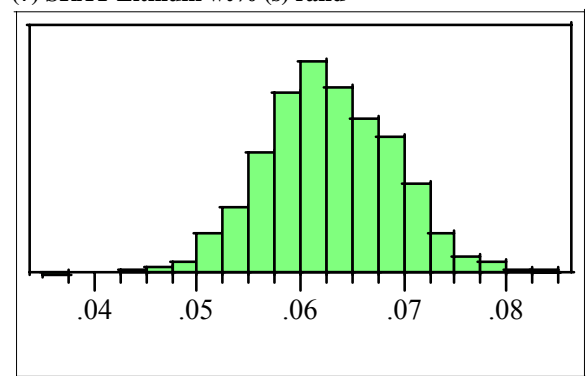

Moments

Mean

Std Err Mean

upper 95\% Mean

0.0628596

0.006414

0.0002028

0.0632577

1000

(7) SRAT Magnesium wt \% (s) rand

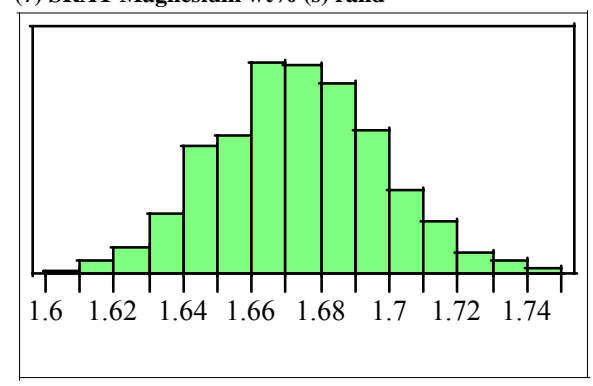

Moments

Mean

Std Err Me

upper 95\% Mean

lower $95 \%$ Mea

1.6735341

0.0007707

0.000707
1.6750465

1.6750465
1.6720217

1000 
Exhibit A6. Histograms and Other Descriptive Statistics for Inputs to WL Targeting Assuming Nominal Number of Samples

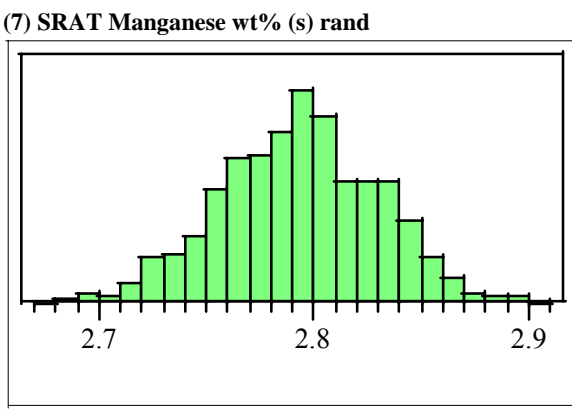

Moments

Mean

Std Dev

upper $95 \%$ Me

lower $95 \%$ Mean

2.7928983

0.037048

0.0011716
2.7951973

2.7951973

(7) SRAT Sodium wt\% (s) rand

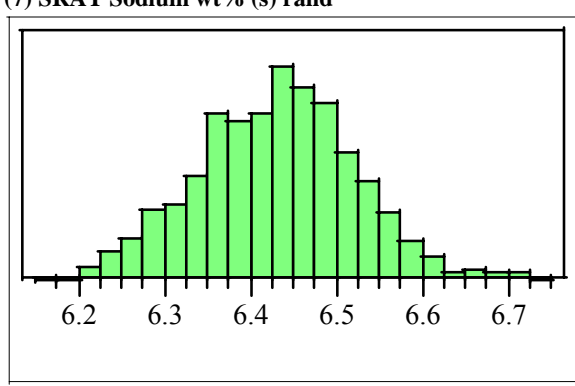

Moments

Mean

Std Dev

lower 95\% Mea

6.4305032

0.0907308

0.0028692

6.4361335

1000
(7) SRAT Nickel wt \% (s) rand

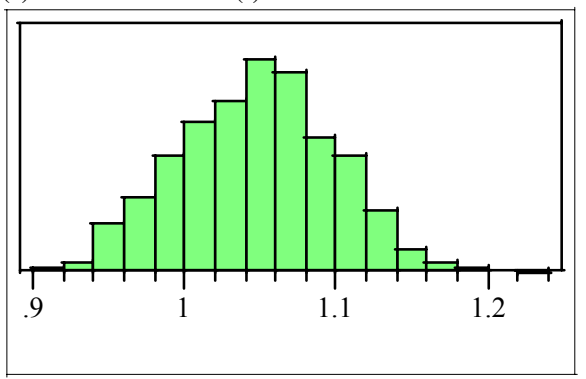

Moments

Mean

Std Err Mean

St Er $95 \%$ Mean

upper $95 \%$ Mean

(7) SRAT Silicon wt\% (s) rand

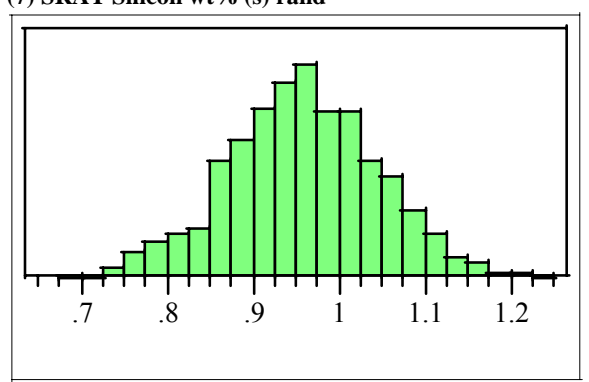

Moments

Mean

Std Err Mean

upper $95 \%$ Mea

$\mathrm{N}$
(7) SRAT Titanium wt\% (s) rand

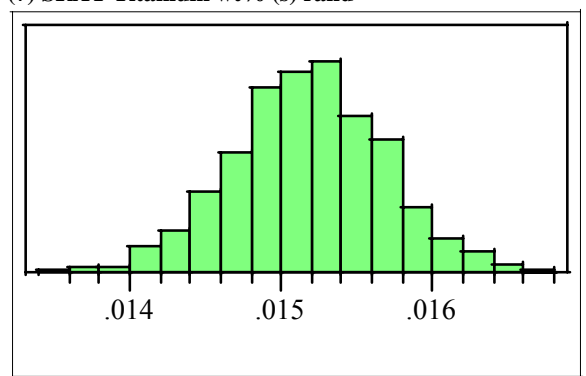

Moments

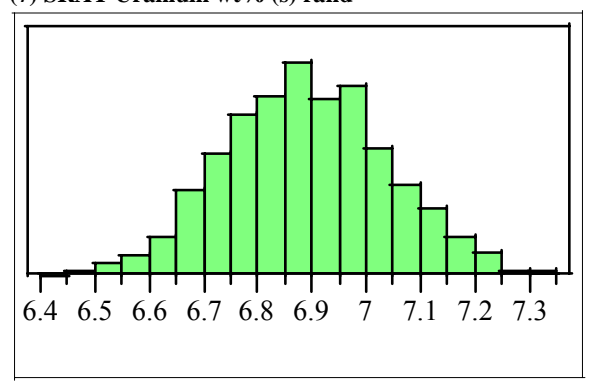

1.0470837

0.050428

0.001594

1.050213

Mean

Std Err Mean

upper $95 \%$ Mean

upper $95 \%$ Mean

(7) SRAT Uranium wt $\%$ (s) rand

Moments

Mean

Std Err Mean

upper $95 \%$ Mean

lower $95 \%$ Mea

0.0151768

0.0005074

0.000016

0.0151453

1000

0.0869347

0.964313

1000

6.8888818

0.1491283

0.0047159
6.8981359

6.8981359
6.8796277

1000 
Exhibit A6. Histograms and Other Descriptive Statistics for Inputs to WL Targeting Assuming Nominal Number of Samples

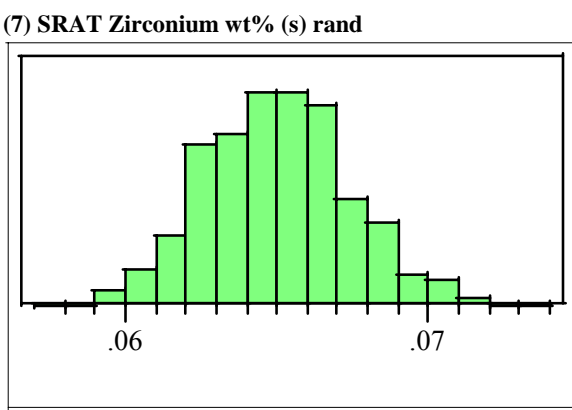

Moments

Mean

Std Err Mea

Std Err Mean

upper $95 \%$ Mean

0.0650175

0.002368

0.0000749

0.0651645

1000

(8) SRAT Volume (gals) rand

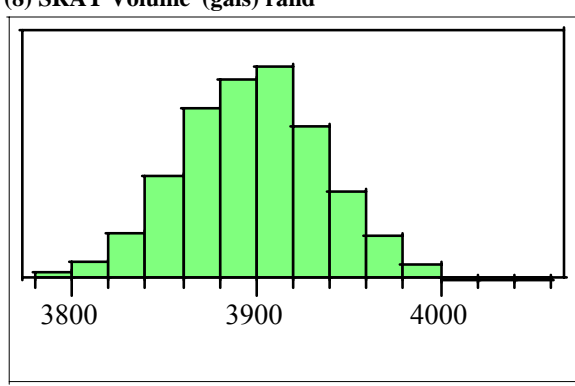

Moments

Mean

Std Dev

lower $95 \%$ Mea

ower $95 \%$ Mea

3898.2007

38.986737

1.2328689

3900.62
(9) Frit 320 Aluminum wt\% (v) rand

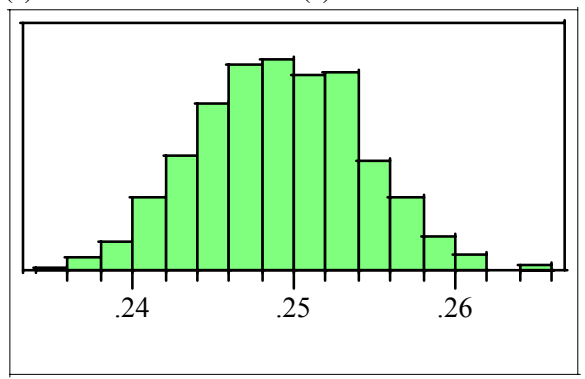

Moments

Mean

Std Err Mean

upper $95 \%$ Mean

upper $95 \%$ Mean

0.2491261

0.0050682

0.2494406

(9) Frit 320 Boron wt \% (v) rand

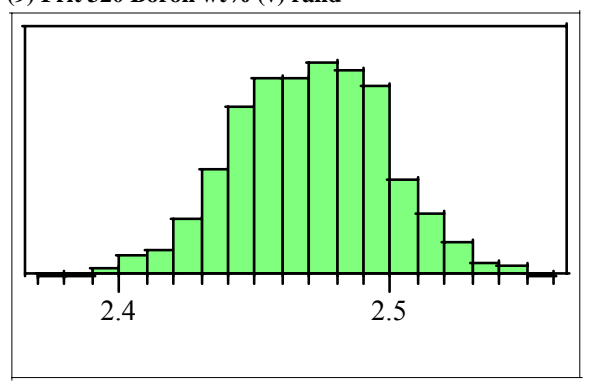

Moments

Mean

Std Err Mean

upper $95 \%$ Mean

upper $95 \%$ Mea

$\mathrm{N}$

2.472004

1000
(9) Frit 320 Calcium wt \% (v) rand

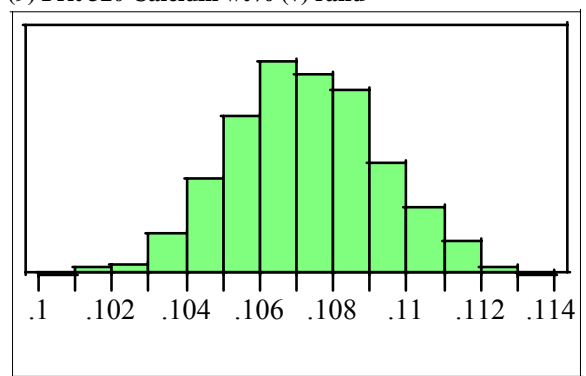

Moments

Mean

Std Err Mean

upper $95 \%$ Mean

0.1072404

0.0000643

0.1073667

0.1073667

(9) Frit 320 Chromium wt \% (v) rand

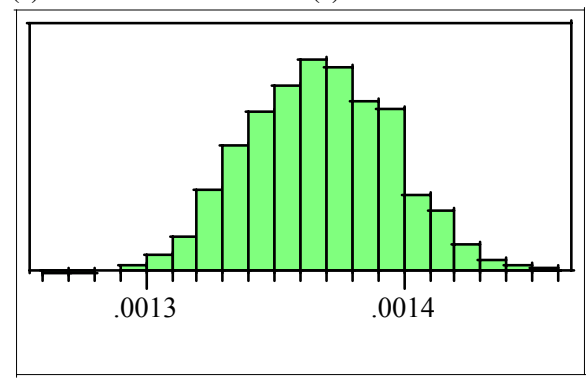

Moments

Mean

0.0013675

Std Dev

upper $95 \%$ Mea

lower $95 \%$ Mea

0.000028

$8.9531 \mathrm{e}-7$

0.0013693

1000 
Exhibit A6. Histograms and Other Descriptive Statistics for Inputs to WL Targeting Assuming Nominal Number of Samples

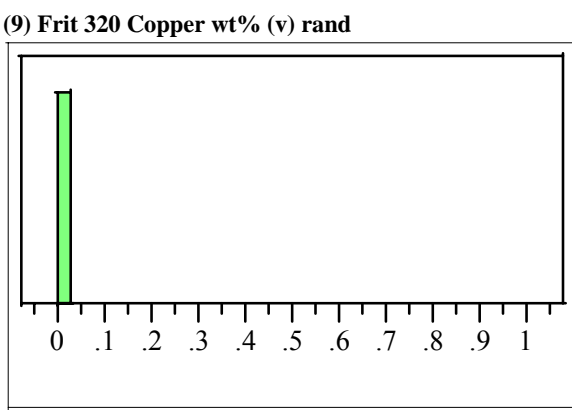

Moments

Mean

Std Dev

Std Err Mean

upper $95 \%$ Mean

ower $95 \%$ Mean

(9) Frit 320 Iron wt \% (v) rand

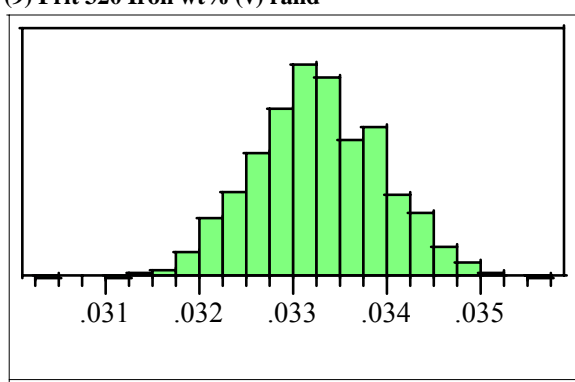

Moments

Mean

0.0332671

Std Dev

lower $95 \%$ Mea

0.0006818

0.0000216

1000
(9) Frit 320 Potassium wt\% (v) rand

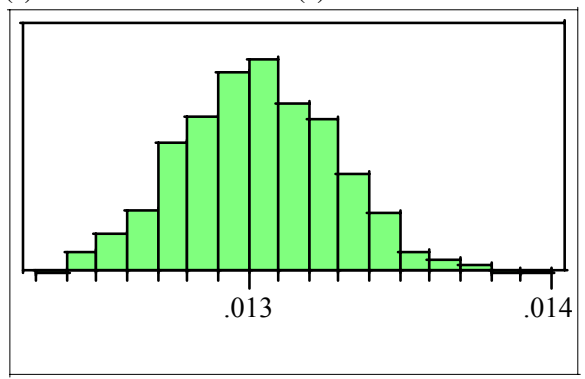

Moments

Mean

Std Err Mean

St Er $95 \%$ Mean

upper $95 \%$ Mea

0.0130336

0.0002525

$$
1000
$$

(9) Frit 320 Lithium wt \% (v) rand

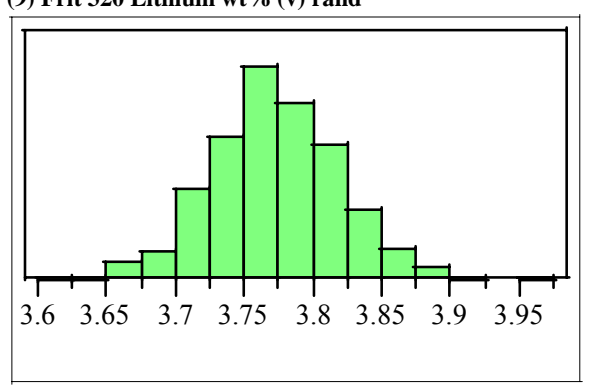

Moments

Mean

Std Err Mean

upper $95 \%$ Mean

lower $95 \%$ Mean

3.772915

0.0460263

0.0014555

3.7700593

1000
(9) Frit 320 Magnesium wt\% (v) rand

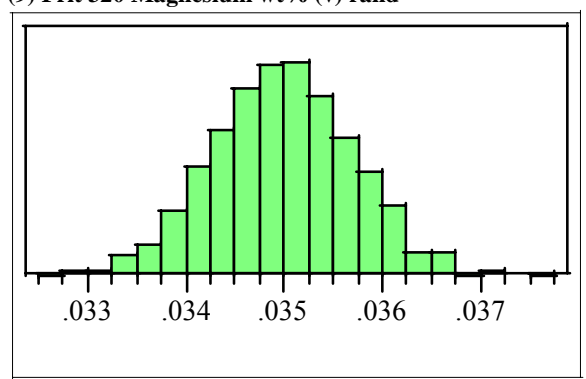

Moments

Mean

Std Err Mean

upper $95 \%$ Mean

lower $95 \%$ Mean

0.0349885

0.0007124

0.000022

0.035032

(9) Frit 320 Manganese wt \% (v) rand

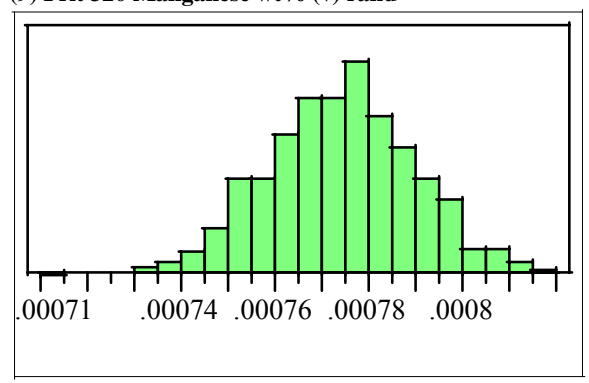

Moments

Mean

Std Err Mean

upper $95 \%$ Mean

lower $95 \%$ Mea

0.000774

0.0000155

$4.9066 \mathrm{e}-7$
0.000775

0.0007731

1000 
Exhibit A6. Histograms and Other Descriptive Statistics for Inputs to WL Targeting Assuming Nominal Number of Samples

(9) Frit 320 Sodium wt $\%$ (v) rand

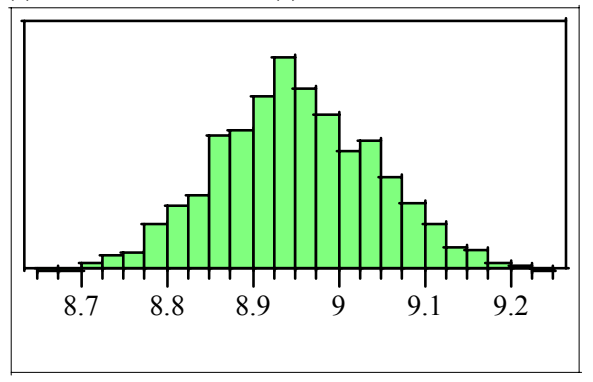

Moments

Mean

Std Err Mea

Std Err Mean

lower $95 \%$ Mean

8.9511029

0.0930499

0.0029425

8.9568771

(9) Frit 320 Nickel wt\% (v) rand

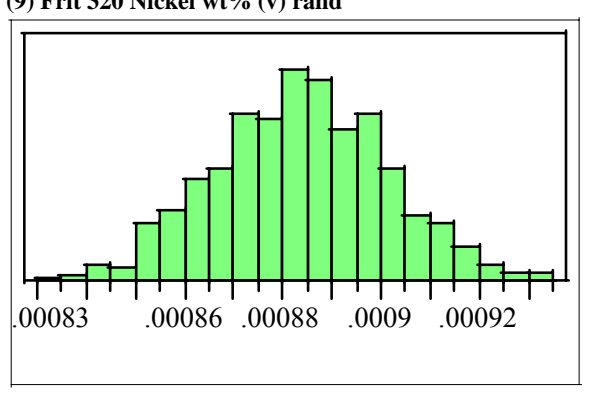

Moments

Mean

Std Dev

upper $95 \%$ Mea

lower $95 \%$ Mean

0.0008838

0.0000176

$5.5725 \mathrm{e}-7$

0.0008849

1000
(9) Frit 320 Silicon wt \% (v) rand

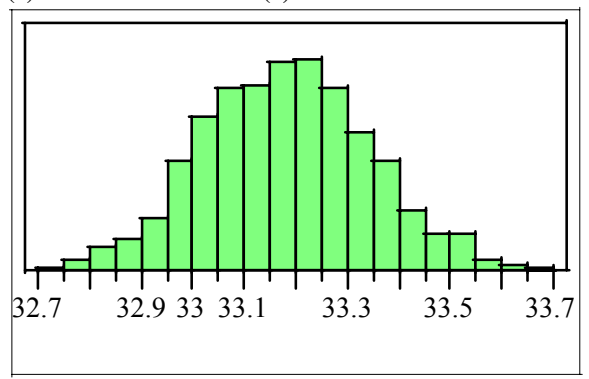

Moments

Mean

Std Err Mean

upper $95 \%$ Mean

upper 95\% Mean

33.179802

0.1627451

0.0051465

$$
1000
$$

(9) Frit 320 Titanium wt\% (v) rand

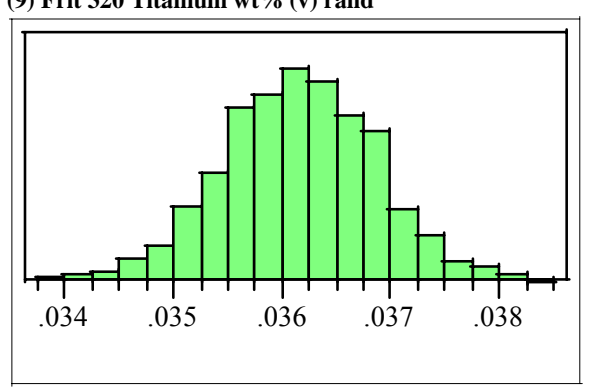

Moments

Mean

Std Dev

Std Err Mean

upper $95 \%$ Mean

lower 95\% Mean
(9) Frit 320 Uranium wt\% (v) ran

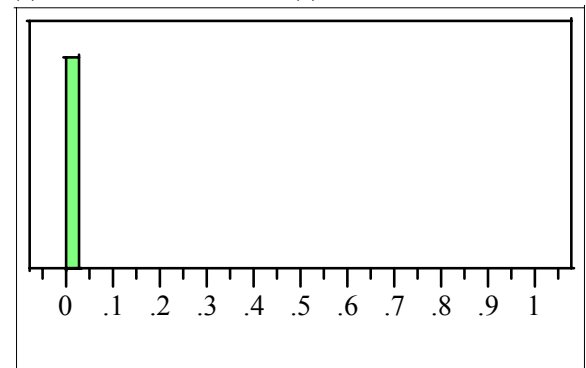

Moments

Mean

Std Err Mean

upper $95 \%$ Mean

(9) Frit 320 Zirconium wt\% (v) rand

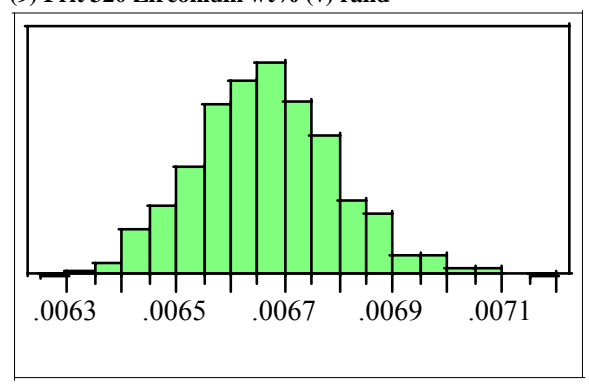

Moments

Mean

Std Err Mean

upper $95 \%$ Mean

lower $95 \%$ Mea

0.0001298

0.0000041

0.0066581

1000 
Exhibit A6. Histograms and Other Descriptive Statistics for Inputs to WL Targeting Assuming Nominal Number of Samples

(10) Frit 320 (lbs) rand

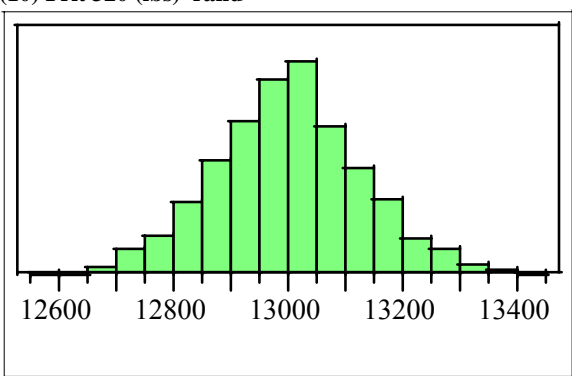

Moments

Mean

Std Dev

Std Err Mean

upper $95 \%$ Mea

$\mathrm{N}$

13000.953

127.48042

4.0312847

13008.864

1000

(11) Frit lbs from 5 Can Decon rand

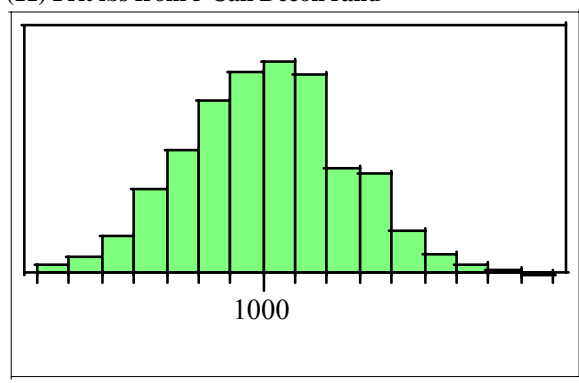

Moments

Mean

1000.6385

1000.6385
24.744295

0.7824833

Std Err Mean $\quad 0.7824833$

upper $95 \%$ Mean 1002.174

ower $95 \%$ Mean 
Exhibit A7. Overview of Uncertainties of Estimated WLs

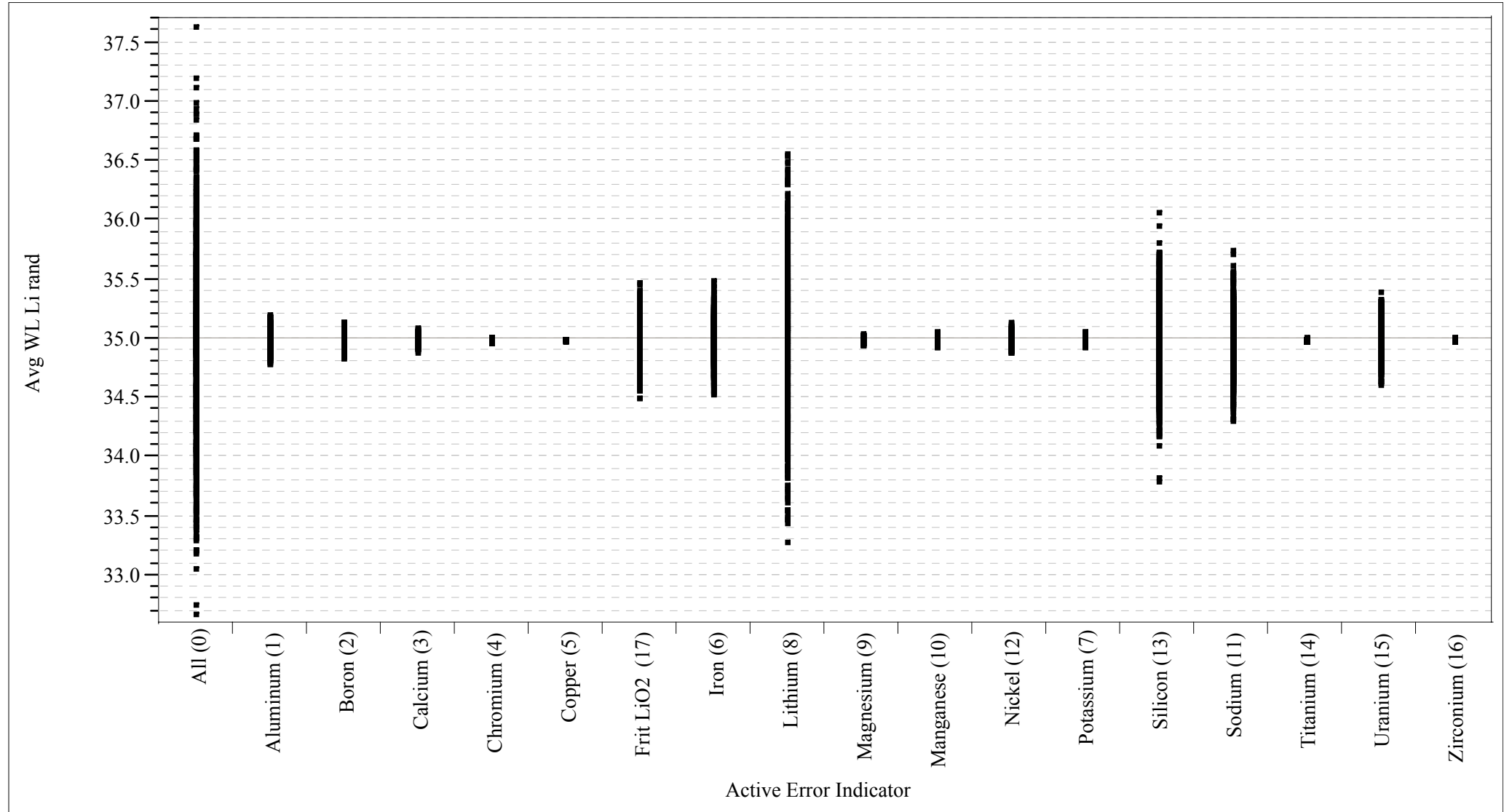




\section{Exhibit A8. Uncertainties of Estimated WLs by Error Number}

Active Error Indicator=All (0)

Distributions

Avg WL Li rand

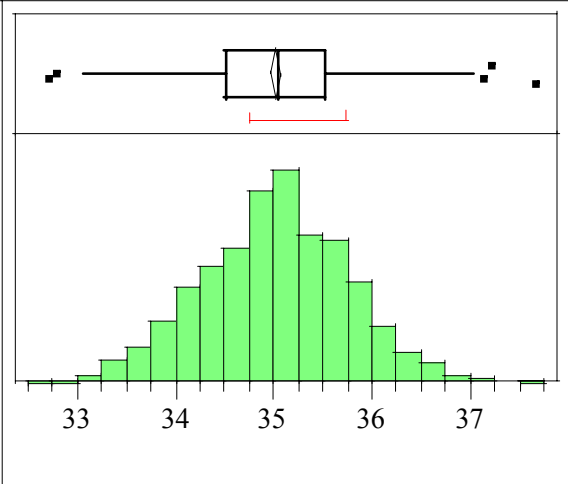

Quantiles

$100.0 \%$ maximum

$99.5 \%$

$97.5 \%$

$90.0 \%$

$75.0 \%$

quartile

$25.0 \%$ quartile

$10.0 \%$

$2.5 \%$

$0.5 \%$

$0.0 \%$

Moments

minimum

Std Dev

Std Err Mean

upper 95\% Mean

lower 95\% Mean

$\mathrm{N}$

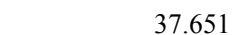

37.651

36.457

35.960

35.529

35.043

34.516

34.045

33.548

33.222

32.689

35.020999

0.7405267

0.0234175

35.066952

34.975046

1000
Active Error Indicator=Aluminum (1)

Distributions

Avg WL Li rand

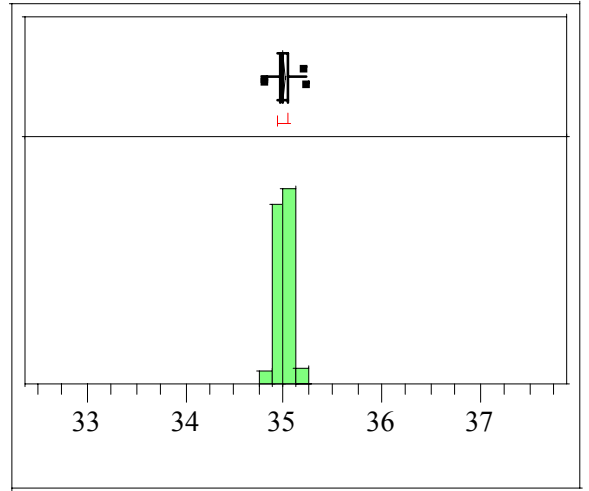

Quantiles

$100.0 \%$ maximum

$99.5 \%$

$97.5 \%$

$90.0 \%$

$75.0 \%$

$50.0 \%$

quartile

$25.0 \%$

median

quartile

$10.0 \%$

$2.5 \%$

$0.5 \%$

Moments

minimum

Mean

Std Dev

Std Err Mean

upper $95 \%$ Mean

lower $95 \%$ Mean

N

35.214

35.197

35.138

35.093

35.053

35.004

34.956

34.913

34.825

34.799

35.004075

0.0701703

0.002219

35.008429

34.99972

1000
Active Error Indicator=Boron (2)

Distributions

Avg WL Li rand

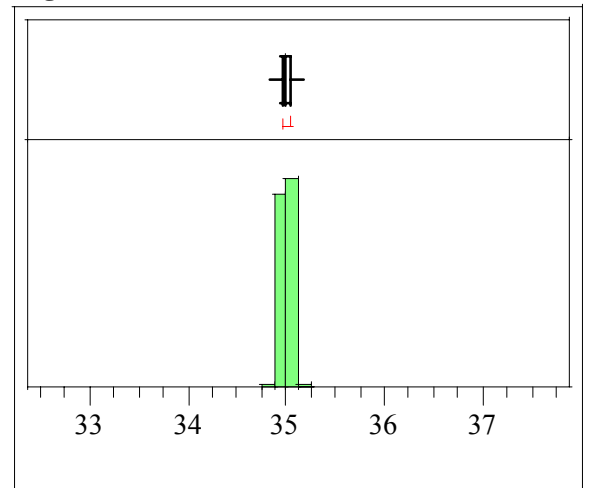

Quantiles

$100.0 \% \quad$ maximum $\quad 35.152$

$99.5 \%$

$97.5 \% \quad 35.107$

$90.0 \% \quad 35.073$

$\begin{array}{ll}75.0 \% & \text { quartile } \\ 50.0 \% & 35.042\end{array}$

$25.0 \%$

$25.0 \%$ quattile

$10.0 \%$

$0.5 \% \quad 34.859$

$0.0 \%$ minimum 34.849

Moments

Mean

35.002269

Std Dev

Std Err Mean

upper $95 \%$ Mean

lower $95 \%$ Mean

0.001729

35.005662

34.998876

1000 
WSRC-TR-2004-00508

Revision 0

\section{Exhibit A8. Uncertainties of Estimated WLs by Error Number}

Active Error Indicator=Calcium (3)

Distributions

Avg WL Li rand

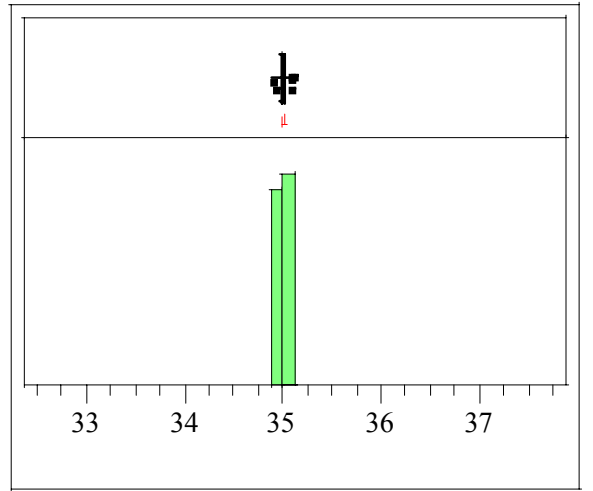

Quantiles

$100.0 \% \quad$ maximum

$99.5 \%$

$97.5 \%$

$90.0 \%$

$75.0 \%$

quartile

$25.0 \%$ quartile

$10.0 \%$

$2.5 \%$

$0.5 \%$

$0.0 \%$

Moments

minimum

Std Dev

Std Err Mean

upper $95 \%$ Mean

lower 95\% Mean

$\begin{array}{ll}\text { maximum } & 35.099 \\ & 35.076 \\ & 35.057 \\ \text { quartile } & 35.038 \\ \text { median } & 35.021 \\ \text { quartile } & 35.002 \\ & 34.982 \\ & 34.964 \\ \text { minimum } & 34.945 \\ & 34.930 \\ & 34.893\end{array}$

35.001302

0.0288808

0.0009133

35.003094

34.99951

1000
Active Error Indicator=Chromium (4)

Distributions

Avg WL Li rand

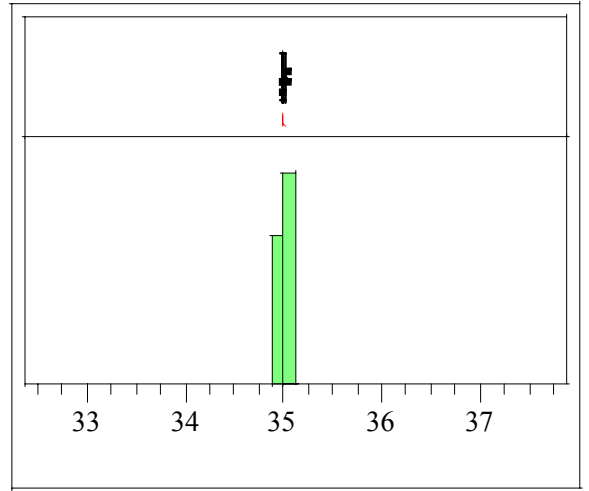

Quantiles

$100.0 \%$ maximum

$99.5 \%$

$97.5 \%$

$90.0 \%$

$75.0 \%$

$50.0 \%$

$25.0 \%$

(a)

quartile

median

quartile

$10.0 \%$

$2.5 \%$

$0.5 \%$

Moments

minimum

Std Dev

Std Err Mean

upper $95 \%$ Mean

lower $95 \%$ Mean

N

35.001543

0.0067785

0.0002144

35.001964

35.001122
Active Error Indicator=Copper (5)

Distributions

Avg WL Li rand

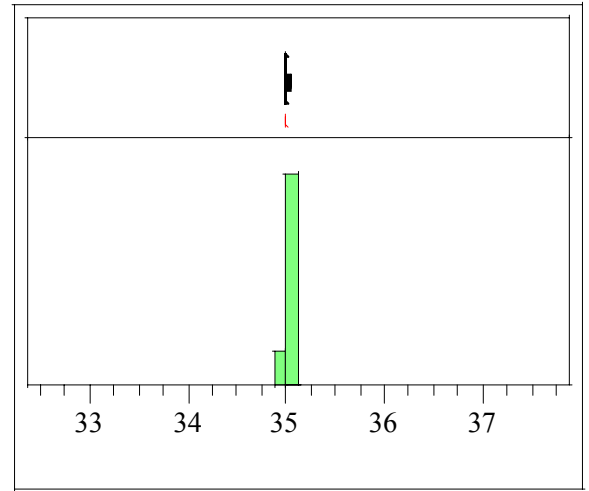

Quantiles

$100.0 \%$

$99.5 \%$

maximum

35.007

$97.5 \%$

$90.0 \%$

$75.0 \%$

$50.0 \%$

$25.0 \%$

$10.0 \%$

$2.5 \%$

$0.5 \%$

$0.0 \%$

Moments

quartile
median
quartile

35.006

35.005

35.003

35.002

35.001

35.000

34.999

34.998

Mean

Std Dev

Std Err Mean

upper $95 \%$ Mean

lower $95 \%$ Mea

N

35.001729

0.0016021

0.0000507

35.001828

35.001629 


\section{Exhibit A8. Uncertainties of Estimated WLs by Error Number}

Active Error Indicator=Frit Li2O (17)

Distributions

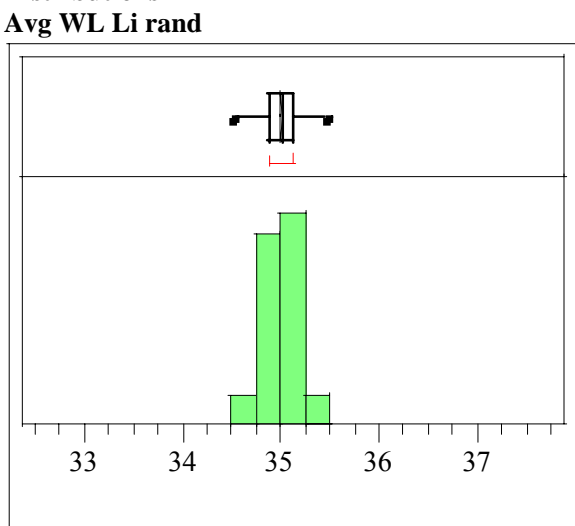

Quantiles

$100.0 \%$ maximum

$99.5 \%$

$97.5 \%$

$90.0 \%$

$75.0 \%$

quartile

$25.0 \%$ quartile

$10.0 \%$

$2.5 \%$

$0.5 \%$

$0.0 \%$

Moments

minimum

Std Dev

Std Err Mean

upper $95 \%$ Mean

lower 95\% Mean

$\begin{array}{lr} & 35.492 \\ & 35.397 \\ & 35.309 \\ \text { quartile } & 35.215 \\ \text { median } & 35.119 \\ \text { quartile } & 35.009 \\ & 34.891 \\ & 34.785 \\ \text { minimum } & 34.684 \\ & 34.587 \\ & 34.503 \\ & \\ & 35.003879 \\ & 0.1633252 \\ & 0.0051648 \\ \text { an } & 35.014014 \\ & 34.993743 \\ & 1000\end{array}$

1000
Active Error Indicator=Iron (6)

Distributions

Avg WL Li rand

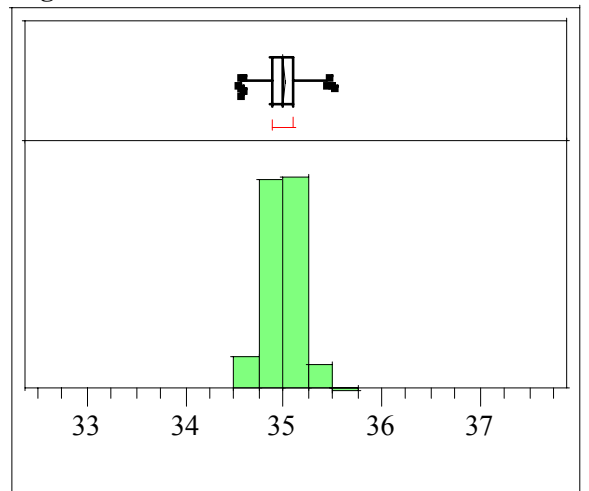

Quantiles

$100.0 \%$ maximum

$99.5 \%$

$97.5 \%$

$90.0 \%$

$75.0 \%$

$50.0 \%$

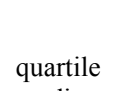

$25.0 \%$

median

quartile

$10.0 \%$

$2.5 \%$

$0.5 \%$
$0.0 \%$

Moments

minimum

Std Dev

Std Err Mean

upper $95 \%$ Mean

lower $95 \%$ Mean

$\mathrm{N}$
35.502

35.413

35.298

35.197

35.111

34.998

34.897

34.684

34.570

34.541

34.999114

0.1590175

0.0050286

35.008982

34.989246

1000
Active Error Indicator=Lithium (8)

Distributions

Avg WL Li rand

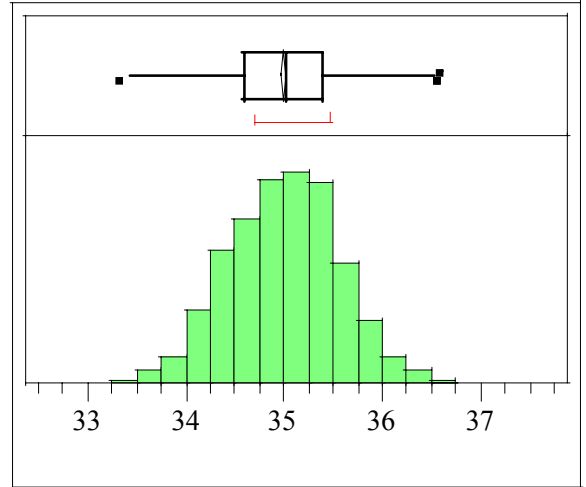

Quantiles

$100.0 \% \quad$ maximum $\quad 36.573$

$99.5 \%-36.489$

$\begin{array}{ll}97.5 \% & 36.108\end{array}$

$90.0 \%$

$75.0 \%$ quartile $\quad 35.379$

$50.0 \%$ median 35.011

$25.0 \%$ quartile 34.603

$10.0 \% \quad 34.256$

$2.5 \% \quad 33.901$

$0.5 \% \quad 33.502$

$0.0 \%$ minimum 33.296

Moments

Mean

34.994933

Std Dev

Std Err Mean

upper $95 \%$ Mean

lower $95 \%$ Mea

0.0179729

35.030202

34.959664 
WSRC-TR-2004-00508

Revision 0

\section{Exhibit A8. Uncertainties of Estimated WLs by Error Number}

Active Error Indicator=Magnesium (9)

Distributions

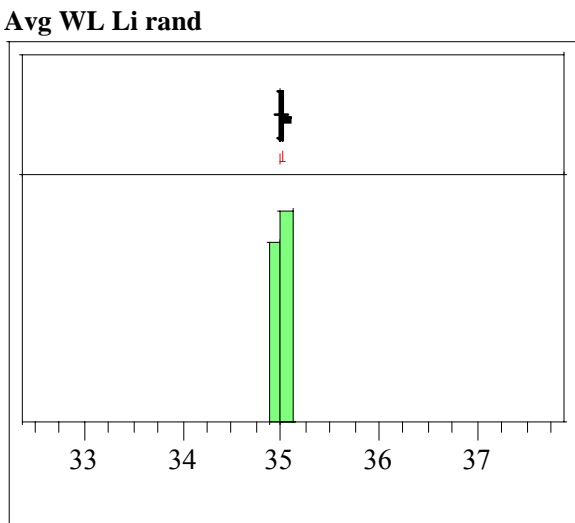

Quantiles

$100.0 \%$ maximum

$99.5 \%$

$97.5 \%$

$90.0 \%$

$75.0 \%$

quartile

$25.0 \%$ quartile

$10.0 \%$

$2.5 \%$

$0.5 \%$

$0.0 \%$

Moments

minimum

Std Dev

Std Err Mean

upper $95 \%$ Mean

lower 95\% Mean

$\begin{array}{ll} & 35.041 \\ & 35.032 \\ & 35.023 \\ \text { quartile } & 35.014 \\ \text { median } & 35.002 \\ \text { quartile } & 34.991 \\ & 34.982 \\ & 34.972 \\ \text { minimum } & 34.964 \\ & 34.960\end{array}$

35.002167

0.0157439

0.0004979

35.003144

35.00119

1000
Active Error Indicator=Manganese (10)

Distributions

Avg WL Li rand

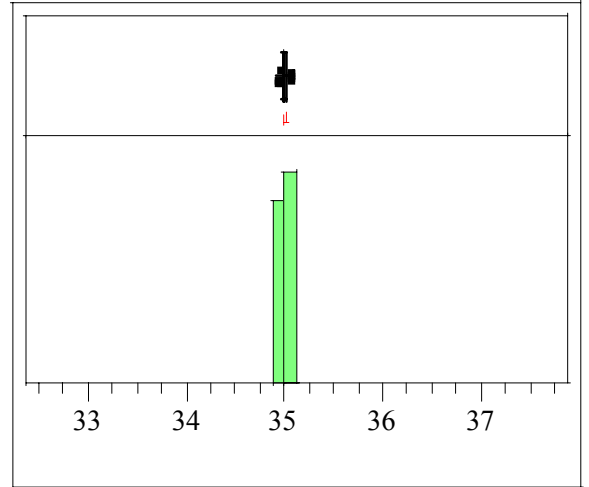

Quantiles

$100.0 \%$ maximum

$99.5 \%$

$97.5 \%$

$90.0 \%$

$75.0 \%$

$50.0 \%$

(2)

quartile

median

$25.0 \%$ quartile

$10.0 \%$

$2.5 \%$

$0.5 \%$

$0.0 \%$

minimum

Moments

Mean

Std Err Mean

upper $95 \%$ Mean

upper $95 \%$ Mean

$\mathrm{N}$

35.001079
Active Error Indicator=Nickel (12)

Distributions

Avg WL Li rand

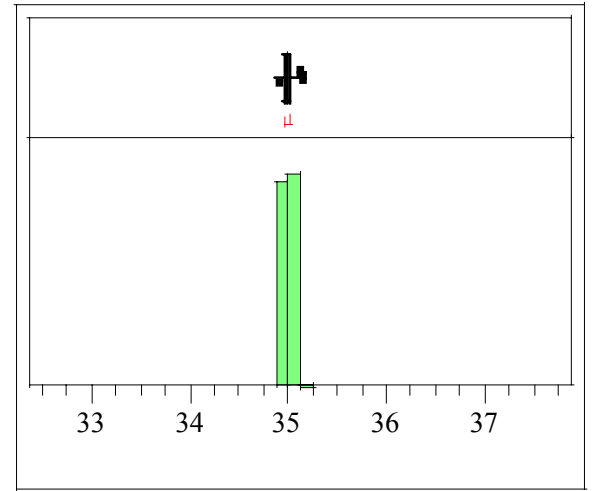

Quantiles

$100.0 \% \quad$ maximum $\quad 35.147$

$99.5 \% \quad 35.102$

$97.5 \%$

$90.0 \%$

$\begin{array}{lll}75.0 \% & \text { quartile } & 35.029 \\ 50.0 \% & 35.001\end{array}$

34.975

$10.0 \% \quad$ quartile

$10.0 \%-2 .-10 \%$

$\begin{array}{ll}2.5 \% & 34.904\end{array}$

$0.0 \%$ minimum 34.894

Moments

Mean

35.001728

Std Dev

Std Err Mean

upper $95 \%$ Mean

lower $95 \%$ Mean

0.039562

0.0012511

35.004183

34.999273

N 


\section{Exhibit A8. Uncertainties of Estimated WLs by Error Number}

Active Error Indicator=Potassium (7)

Distributions

Avg WL Li rand

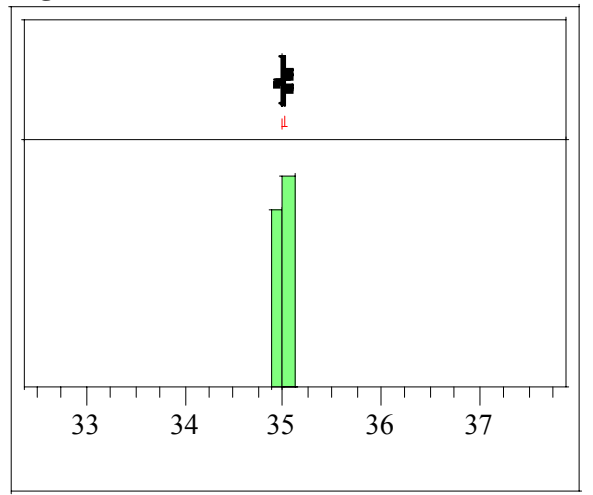

Quantiles

$100.0 \%$ maximum

$99.5 \%$

$97.5 \%$

$90.0 \%$

$75.0 \%$

quartile

$25.0 \%$ quartile

$10.0 \%$

$2.5 \%$

$0.5 \%$

$0.0 \%$

Moments

minimum

Std Dev

Std Err Mean

upper $95 \%$ Mean

lower 95\% Mean

$\begin{array}{ll} & 35.071 \\ & 35.058 \\ & 35.047 \\ \text { quartile } & 35.030 \\ \text { median } & 35.017 \\ \text { quartile } & 35.003 \\ & 34.988 \\ & 34.975 \\ \text { minimum } & 34.960 \\ & 34.948 \\ & 34.938\end{array}$

35.002731

0.021576

0.0006823

35.00407

35.001392

1000
Active Error Indicator=Silicon (13)

Distributions

Avg WL Li rand

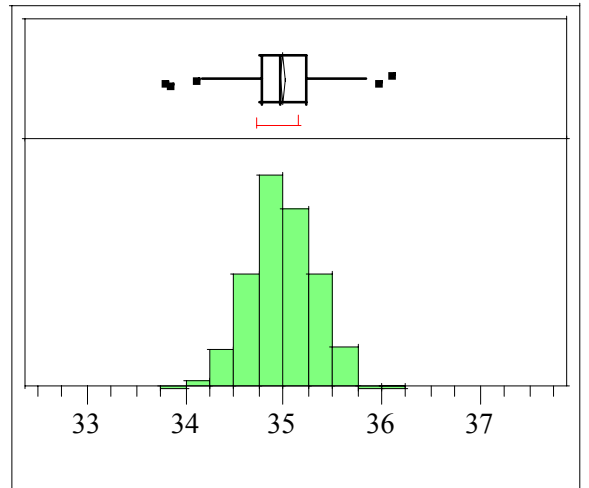

Quantiles

$100.0 \%$ maximum

$99.5 \%$

$97.5 \%$

$90.0 \%$

$75.0 \%$

$50.0 \%$

$25.0 \%$

(2)

quartile

median

quartile

$10.0 \%$

$2.5 \%$

$0.5 \%$

$0.0 \%$

minimum

Moments

Mean

Std Err Mean

upper $95 \%$ Mean

upper $95 \%$ Mean

$\mathrm{N}$

34.973029
Active Error Indicator=Sodium (11)

Distributions

Avg WL Li rand

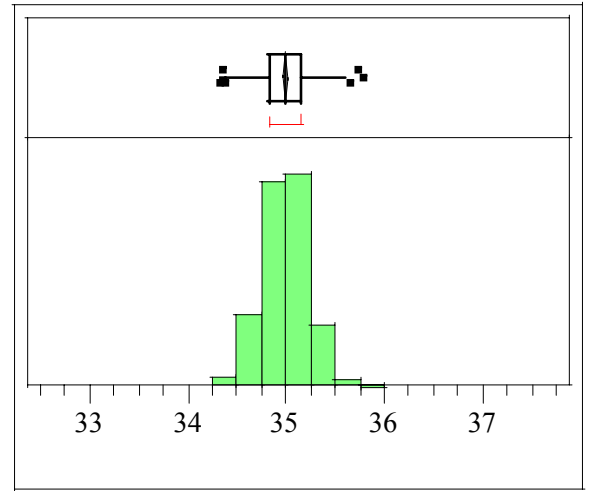

Quantiles

$100.0 \% \quad$ maximum $\quad 35.765$

$99.5 \% \quad 35.566$

$97.5 \% \quad 35.409$

$90.0 \%$

$\begin{array}{ll}75.0 \% & \text { quartile } \\ 50.0 \% & 35.144\end{array}$

34.840

$25.0 \%$ quartile

$\begin{array}{ll}10.0 \% & 34.695 \\ 2.5 \% & 34.546\end{array}$

$\begin{array}{ll}2.5 \% & 34.404\end{array}$

$0.0 \%$ minimum 34.325

Moments

Mean

34.992592

Std Dev

Std Err Mean

upper $95 \%$ Mean

lower $95 \%$ Mean

0.007123

35.00657

34.978615

$\mathrm{N}$ 
WSRC-TR-2004-00508

Revision 0

\section{Exhibit A8. Uncertainties of Estimated WLs by Error Number}

Active Error Indicator=Titanium (14)

Distributions

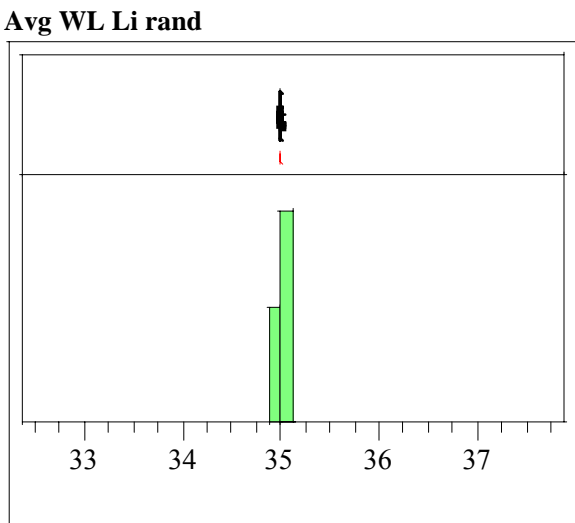

Quantiles

$100.0 \%$

$99.5 \%$

$97.5 \%$

$90.0 \%$

$75.0 \%$

quartile

$25.0 \%$ quartile

$10.0 \%$

$2.5 \%$

$0.5 \%$

$0.0 \%$

Moments

minimum

Std Dev

Std Err Mean

upper 95\% Mean

lower 95\% Mean

$\begin{array}{ll}\text { maximum } & 35.015 \\ & 35.013 \\ & 35.011 \\ \text { quartile } & 35.008 \\ \text { median } & 35.005 \\ \text { quartile } & 35.002 \\ & 34.999 \\ & 34.996 \\ & 34.993 \\ \text { minimum } & 34.989 \\ & 34.987\end{array}$

35.001903

0.0045992

0.0001454

35.002188

35.001617

1000
Active Error Indicator=Uranium (15)

Distributions

Avg WL Li rand

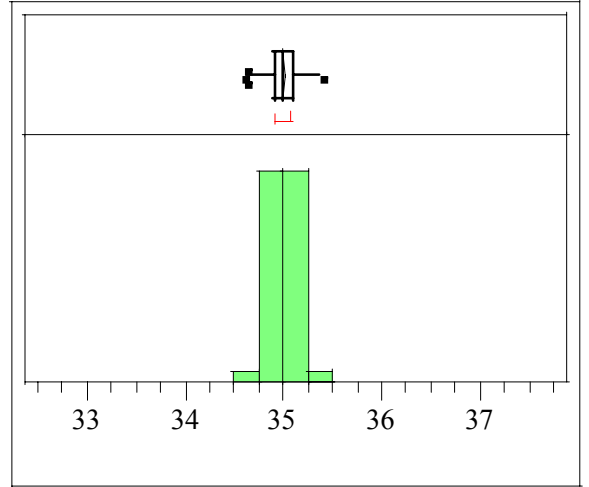

Quantiles

$100.0 \%$ maximum

$99.5 \%$

$97.5 \%$

$90.0 \%$

$75.0 \%$

$50.0 \%$

$25.0 \%$

(a)

quartile

median

quartile

$10.0 \%$

$2.5 \%$

$0.5 \%$

Moments

minimum

Std Dev

Std Err Mean

upper $95 \%$ Mean

lower $95 \%$ Mean

$\mathrm{N}$

1000
Active Error Indicator=Zirconium (16)

Distributions

Avg WL Li rand

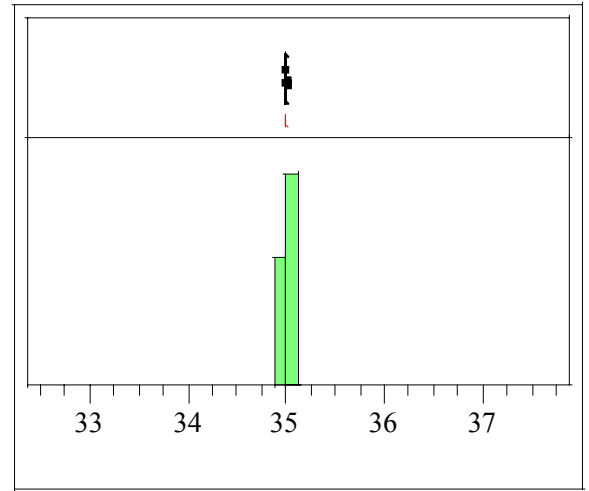

Quantiles

$100.0 \% \quad$ maximum $\quad 35.017$

$99.5 \%$

$97.5 \% \quad 35.01$

35.008

$\begin{array}{ll}75.0 \% & \text { quartile } \\ 50.0 \% & 35.005\end{array}$

$50.0 \%$

$25.0 \%$ quartile 34.998

$10.0 \% \quad 34.995$

$2.5 \%$

$0.0 \%$ minimum 34.986

Moments

Mean

35.001495

Std Dev

Std Err Mean

upper $95 \%$ Mean

lower $95 \%$ Mea

0.004704

0.0001488

35.001786

35.001203

1000 
Error Indicator $=0$

Distributions

1 Aluminum rand

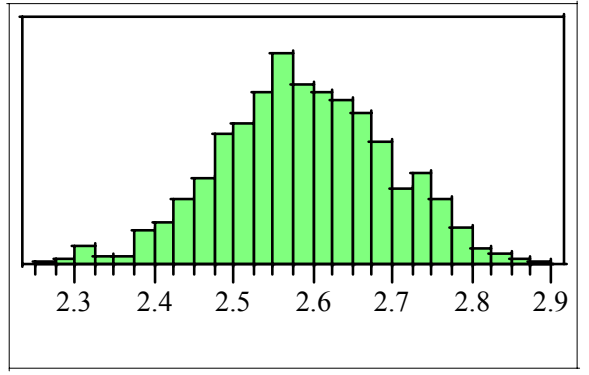

Moments

Mean

Std Dev

upper 95\% Mean

lower 95\% Mean

2.5893669

0.1069462

0.0033819

2.5827304

1000

Sample 1 Boron rand

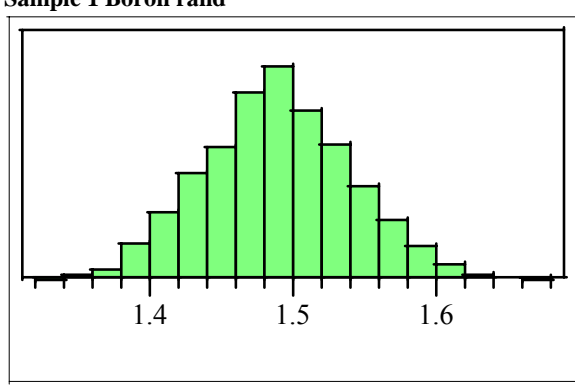

Moments

Mean

Std Dev

upper $95 \%$ Mea

upper $95 \%$ Mea

1.4894601

0.0513924

0.0016252
1.4926493

1.486271

$\mathrm{N}$

\section{Exhibit A9. Uncertainties of Inputs to WL Estimation}

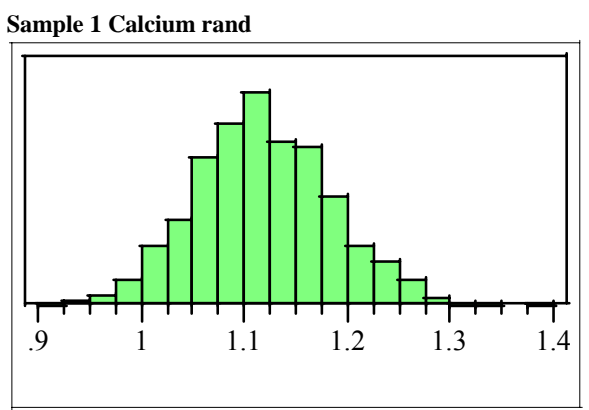

Moments

Mean

Std Err Mean

Std Er Mean

upper $95 \%$ Mea

Sample 1 Chromium rand

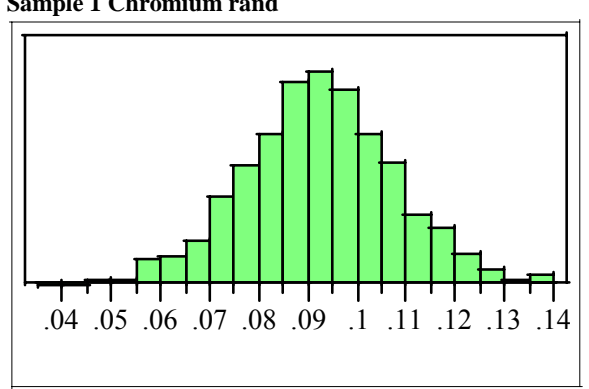

Moments

Mean

Std Err Mean

upper 95\% Mean

upper $95 \%$ Mea
Sample 1 Copper rand

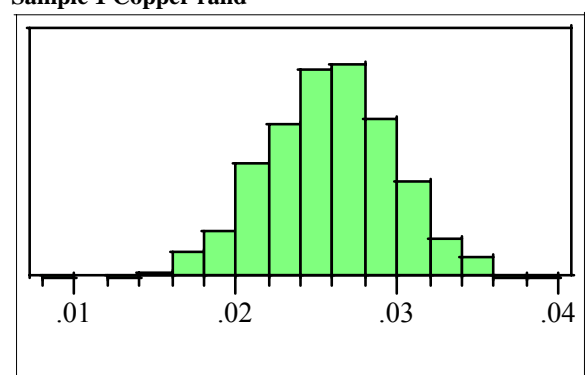

Moments

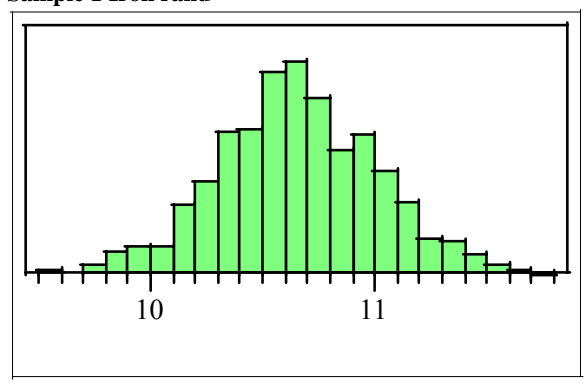

1.1190392

0.0656381

0.002075

1.1231123

1000

Mean

Std Err Mean

upper $95 \%$ Mean

lower $95 \%$ Mean

Sample 1 Iron rand

Moments

Mean

10.65507

Std Dev

upper $95 \%$ Mea

lower $95 \%$ Mea

0.354523

0.011211

10.633071

1000

0.0258161

0.004015

.

$$
1000
$$

0.0004868

$$
1000
$$




\section{Exhibit A9. Uncertainties of Inputs to WL Estimation}

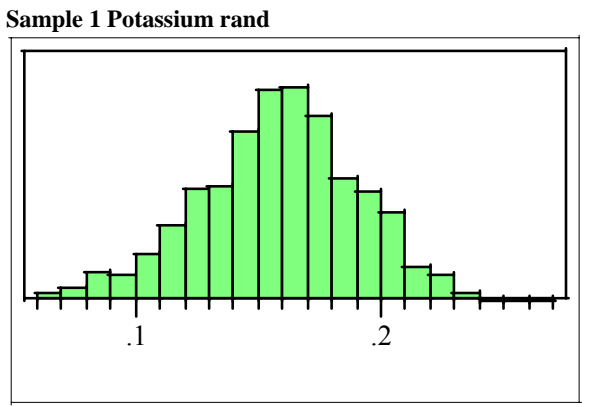

Moments

Mean

Std Dev

Std $95 \%$ Me

lower $95 \%$ Mean

0.158278

0.0322615

0.0010202

0.1602799

Sample 1 Lithium rand

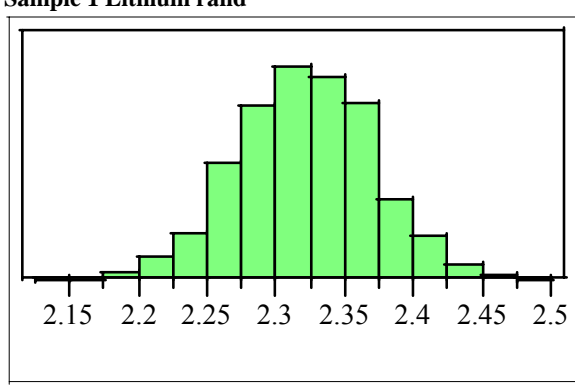

Moments

Mean

Std Dev

upper $95 \%$ Mean

2.3210837

0.0497367

0.0015728

2.324170

1000

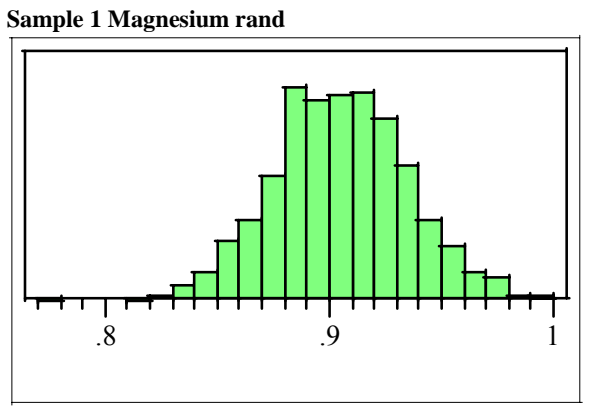

Moments

Mean

Std Err Mean

Sur $95 \%$ Mea

upper $95 \%$ Mea

Sample 1 Manganese rand

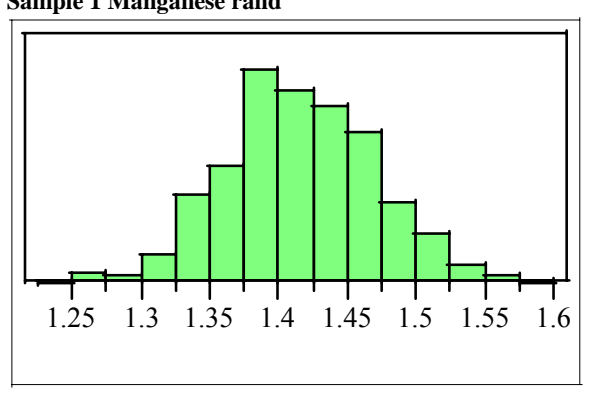

Moments

Mean

Std Dev

upper $95 \%$ Mea

$95 \%$ Mea
Sample 1 Sodium rand

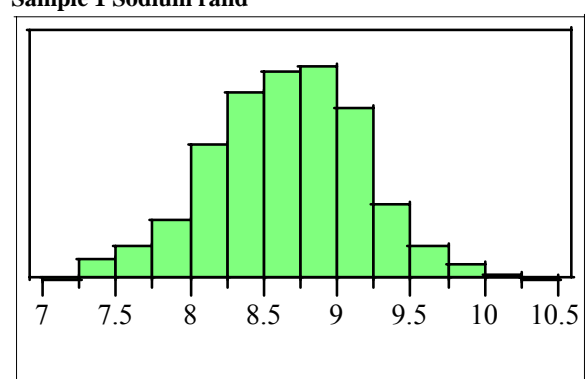

Moments

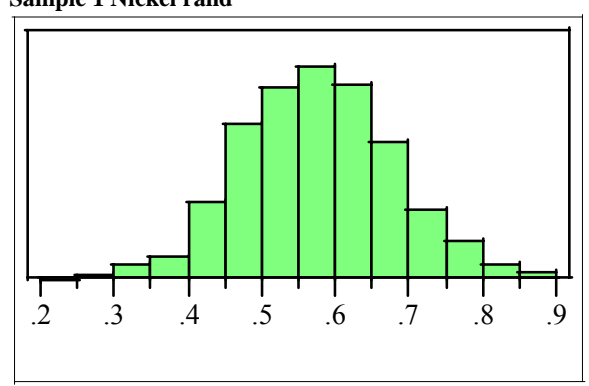

0.904882

0.0295937

0.0009358

0.9067184

1000

Mean

Std Err Mean

upper $95 \%$ Mean

lower $95 \%$ Mean

Sample 1 Nickel rand

Moments

Mean

0.5749857

Std Dev

upper $95 \%$ Mean

lower $95 \%$ Mea

8.6606444

0.511811

0.016184

8.6924047

1000

0.0540309

0.0017086
1.4186942

1000 


\section{Exhibit A9. Uncertainties of Inputs to WL Estimation}

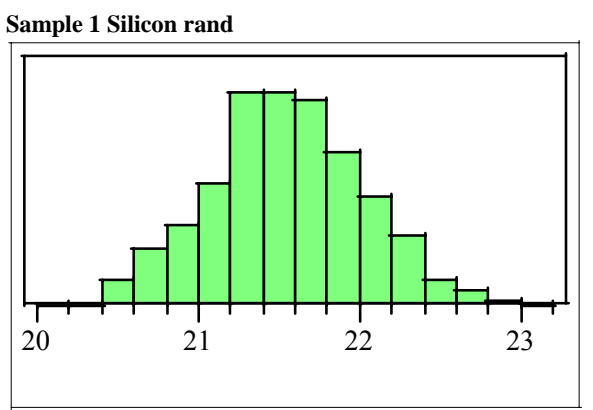

Moments

Mean

Std Dev

Std $95 \%$ Me

lower $95 \%$ Mean

21.552335

0.4753648

21.581834

21.522837

Sample 1 Titanium rand

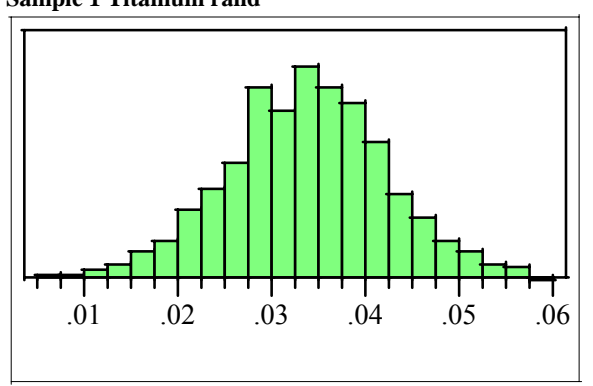

Moments

Mean

Std Dev

upper $95 \%$ Mea

ower 95\% Mean

0.0336882

0.0085425

0.0002701

0.0342183

1000

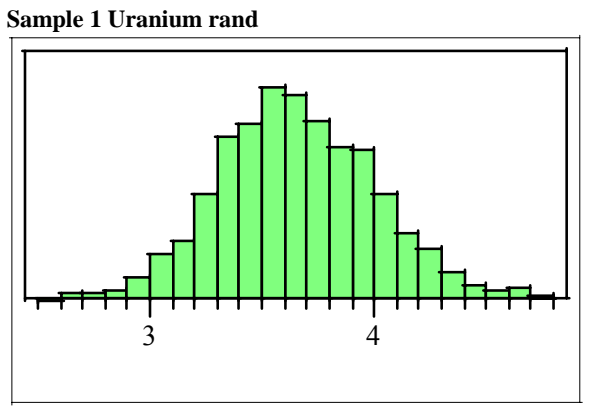

Moments

Mean

Std Err Mean

upper $95 \%$ Mean

upper $95 \%$ Mea

3.6579208

0.3494935

0.011052

3.6796085

1000

Sample 1 Zirconium rand

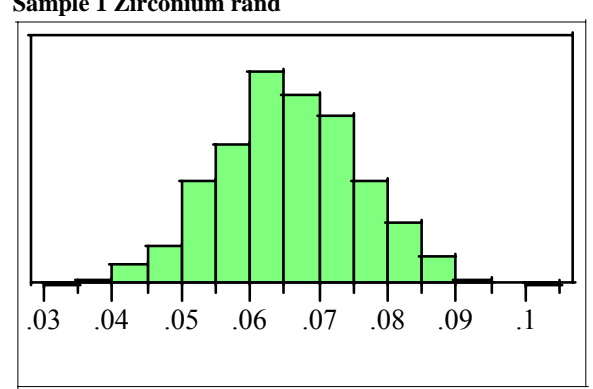

Moments

Mean

Std Dev

Std Err Mean

upper $95 \%$ Mean

lower 95\% Mean

0.0101862

0.0003221

1000
Sample 2 Aluminum rand

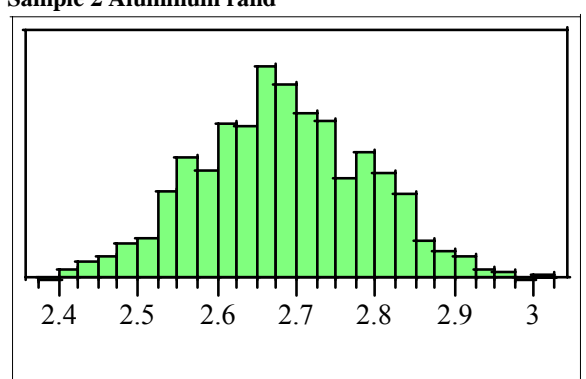

Moments

Mean

Std Err Mean

upper $95 \%$ Mean

2.6828504

0.1075516

0.0690524

2.6895245
2.6761763

Sample 2 Boron rand

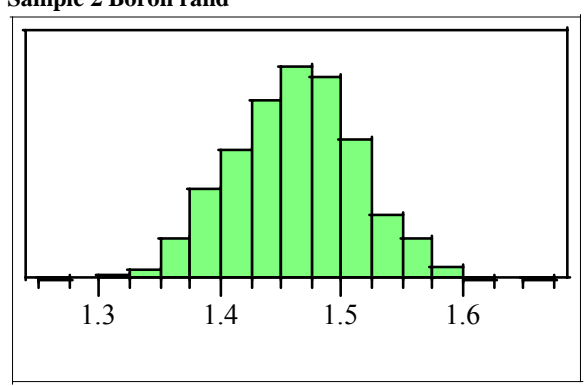

Moments

Mean

Std Err Mean

upper 95\% Mean

lower 95\% Mea

1.4614006

0.0523557

0.0016556
1.4646496

1.4646496
1.4581517

1000 


\section{Exhibit A9. Uncertainties of Inputs to WL Estimation}

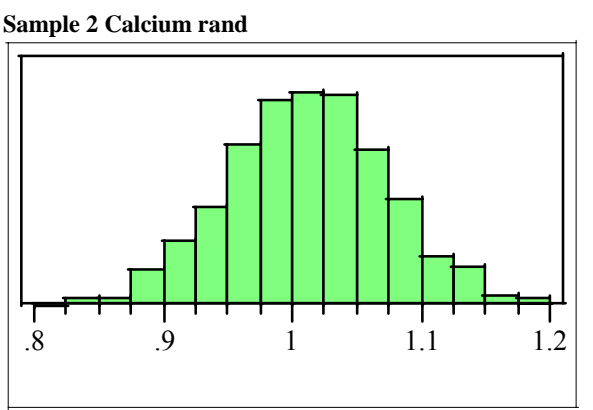

Moments

Mean

Std Dev

Std $95 \%$ Me

lower $95 \%$ Mean

1.0121304

0.0614892

1.0159461

1.0083147

Sample 2 Chromium rand

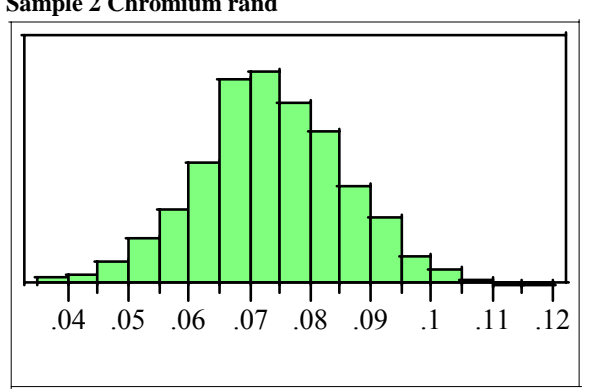

Moments

Mean

Std Dev

upper 95\% Mea

ower 95\% Mean

0.0735538

0.0122432

0.0003872

0.0743135

1000

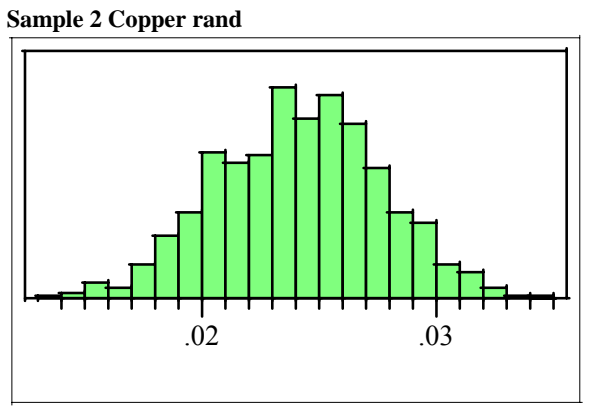

Moments

Mean

Std Dev
Std Err Mean

upper $95 \%$ Mean

upper $95 \%$ Mea

0.0241199

0.0035701

0.0001129

0.0243414

Sample 2 Iron rand

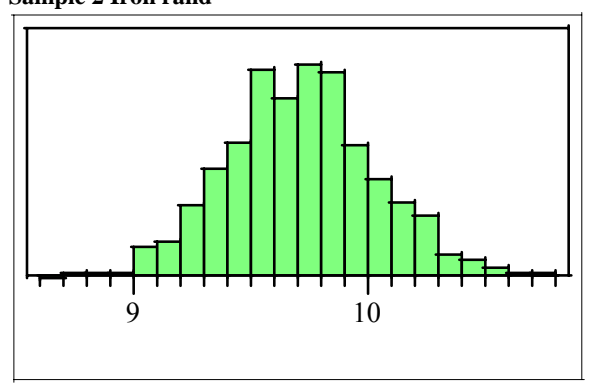

Moments

Mean

Std Dev

upper $95 \%$ Mea

upper $95 \%$ Mea

$5 \%$ Mea

0.316342

0.0100036
9.7362912

9.7362912
Sample 2 Potassium rand

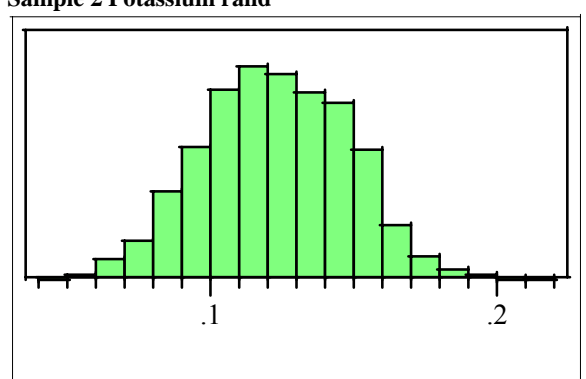

Moments

Mean

Std Err Mean

upper 95\% Mean

0.1225999

0.0252617

0.0007988

0.124167

Sample 2 Lithium rand

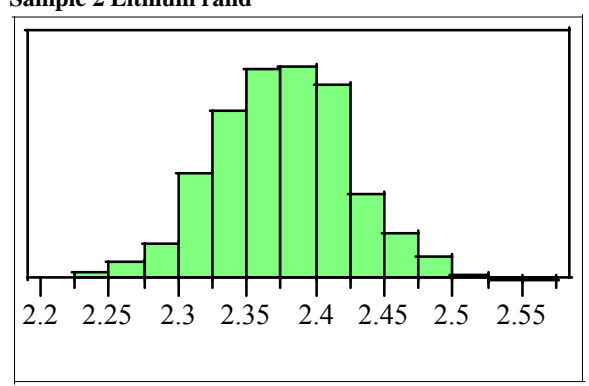

Moments

Mean

Std Err Mean

upper 95\% Mea

lower $95 \%$ Mea

2.3764611

1000

0.050104

0.0015844
2.3795703

2.3733519

1000 


\section{Exhibit A9. Uncertainties of Inputs to WL Estimation}

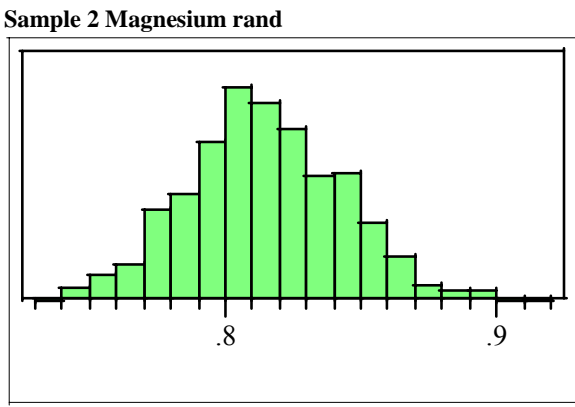

Moments

Mean

Std Dev

Std $95 \%$ Me

lower $95 \%$ Mean

0.8145322

0.0286388

0.8163094

Sample 2 Manganese rand

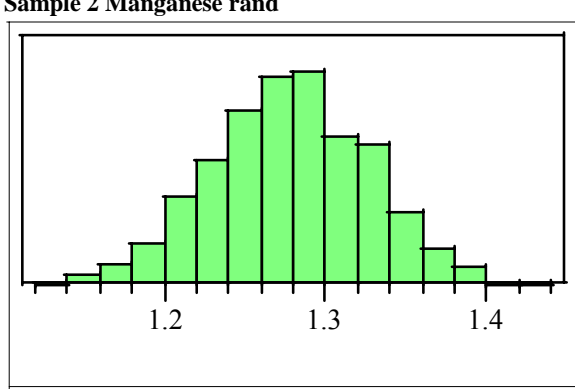

Moments

Mean

Std Dev

upper 95\% Mean

ower 95\% Mean

1.2778164

0.0487013

0.0015401

1.2808385

1000

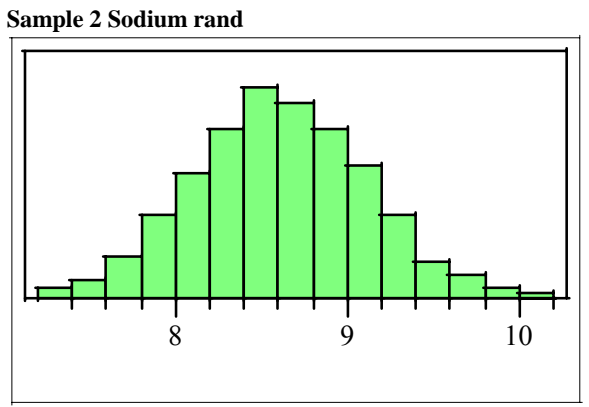

Moments

Mean

Std Err Mean

utd 1 rean

upper $95 \%$ Mea

8.6083959

0.513328

0.0162329

8.6402503

1000

Sample 2 Nickel rand

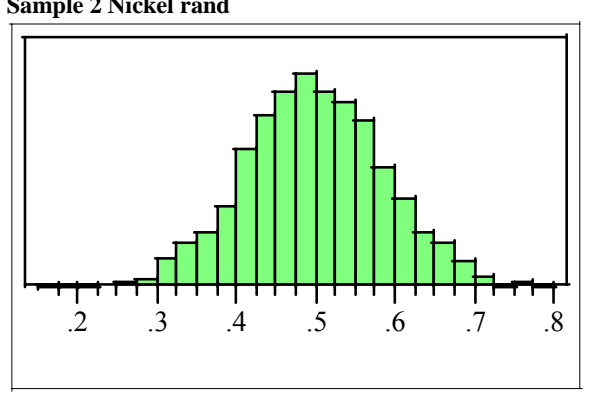

Moments

Mean

Std Dev

Std Err Mean

upper $95 \%$ Mean

lower 95\% Mean

0.4984901

0.0875116

0.0027674

0.4930596

1000
Sample 2 Silicon rand

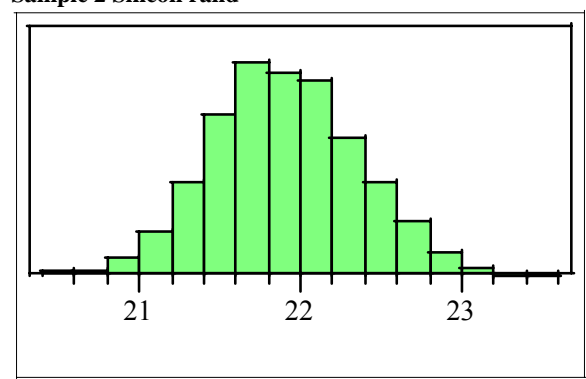

Moments

Mean

Std Err Mean

upper $95 \%$ Mean

upper $95 \%$ Mean
lower $95 \%$ Mean

21.896357

0.4558205

0.014414

21.92464

Sample 2 Titanium rand

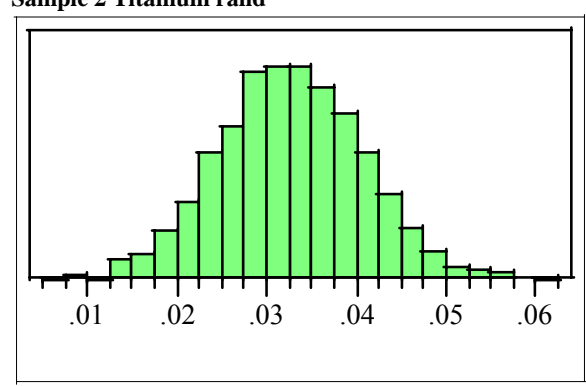

Moments

Mean

0.0326827

Std Dev

upper $95 \%$ Mean

lower 95\% Mea

0.0080251

0.0002538

0.0331807 


\section{Exhibit A9. Uncertainties of Inputs to WL Estimation}

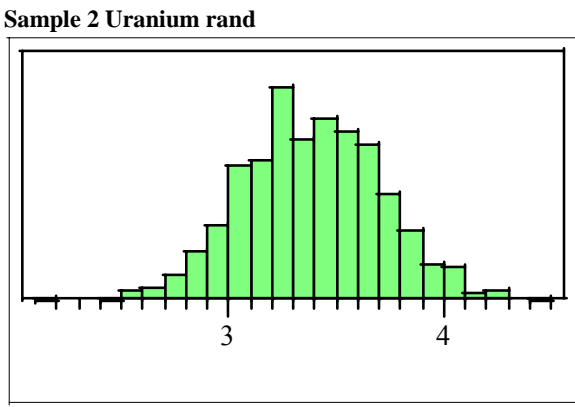

Moments

Mean

Std Dev

Std $95 \%$ Me

lower $95 \%$ Mean

3.3856388

0.3195218

0.0101042

3.4054667

Sample 2 Zirconium rand

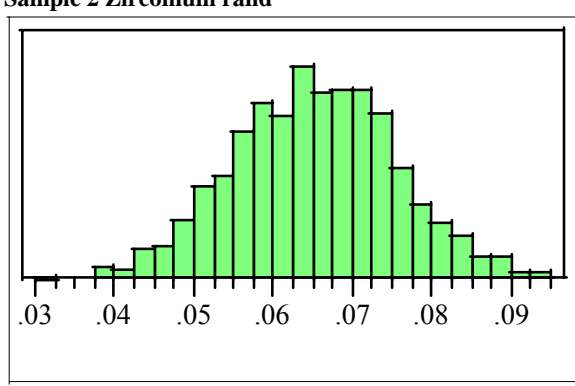

Moments

Mean

Std Dev

upper $95 \%$ Mea

ower 95\% Mean

0.0651991

0.0102212

0.0003232

1000
Sample 3 Aluminum rand

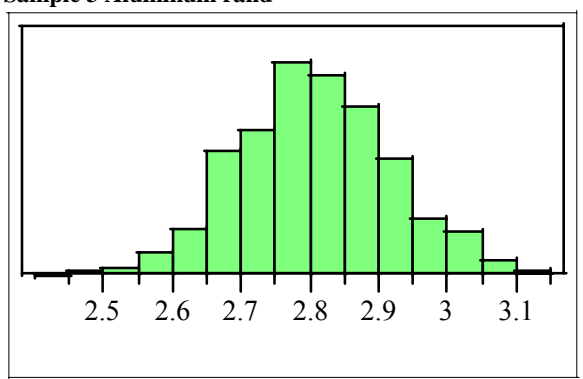

Moments

Mean

Std Err Mean

upper $95 \%$ Mean

upper $95 \%$ Mean

2.8076269

0.1120968

0.003544

2.814583

1000

Sample 3 Boron rand

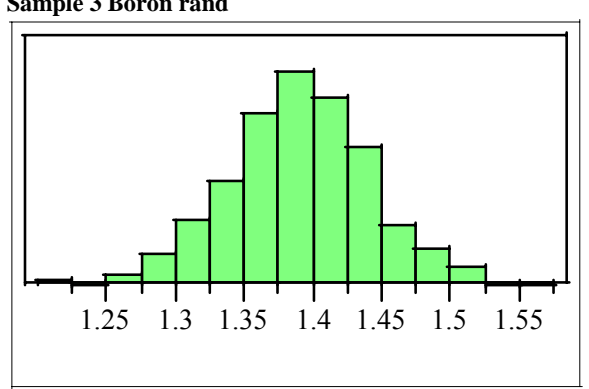

Moments

Mean

Std Err Mean

upper $95 \%$ Mea

$95 \%$ Mea

0.0016248

1.394229
Sample 3 Calcium rand

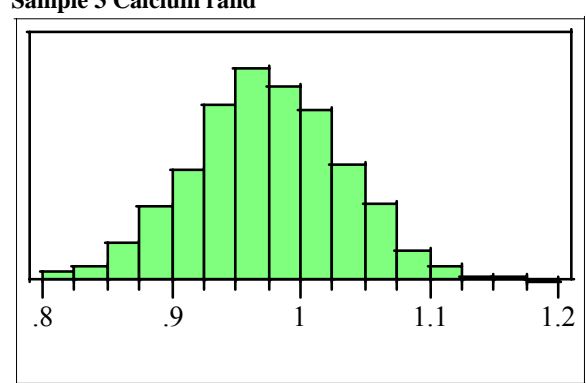

Moments

Mean

Std Err Mean

upper $95 \%$ Mean

lower $95 \%$ Mean

0.9744006

0.059136

0.0780703

0.9780703

Sample 3 Chromium rand

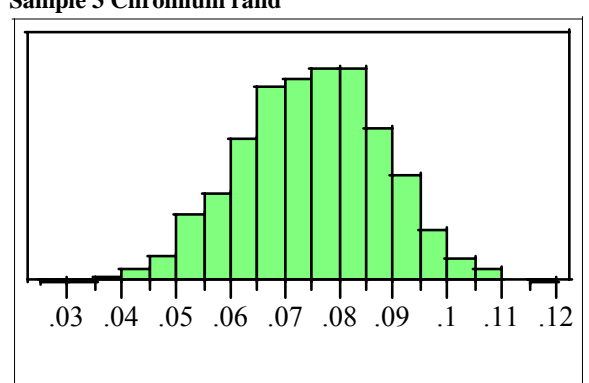

Moments

Mean

0.0749752

Std Dev

upper $95 \%$ Mean

lower 95\% Mea

0.0131037

0.0004144

1000

0.0757884

1000 


\section{Exhibit A9. Uncertainties of Inputs to WL Estimation}

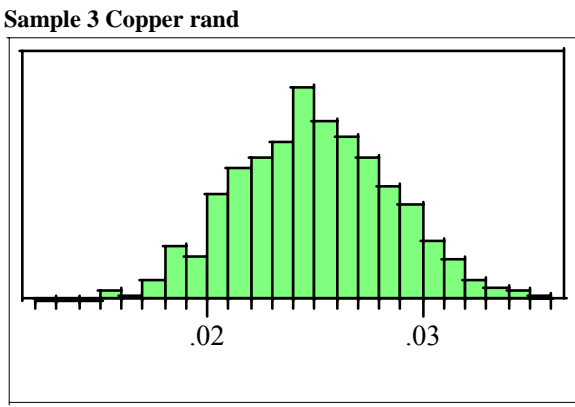

Moments

Mean

Std Err Mea

Std $95 \%$ Me

upper $95 \%$ Mean

0.0249515

0.0036532

0.0251782

0.0251782

Sample 3 Iron rand

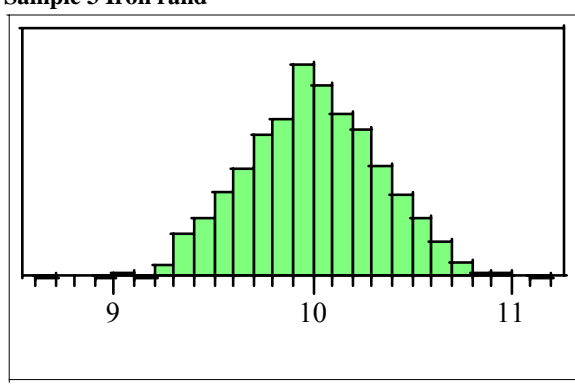

Moments

Mean

Std Dev

upper $95 \%$ Mean

ower 95\% Mean

9.9989525

0.3389558

0.0107187

9. 9779187

1000

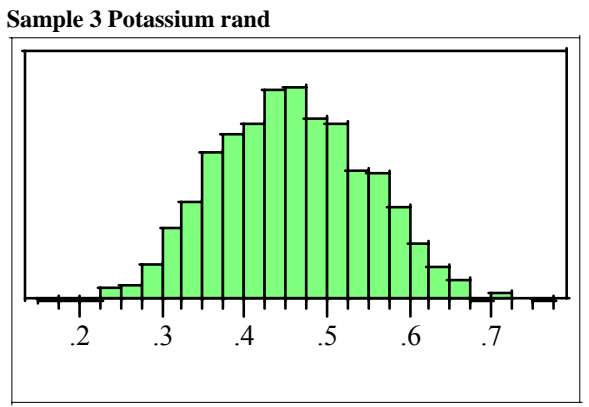

Moments

Mean

Std Dev
Std Err Mean

utd Err Mean

upper $95 \%$ Mea

0.457802

0.0914566

0.002892

0.4634773

Sample 3 Lithium rand

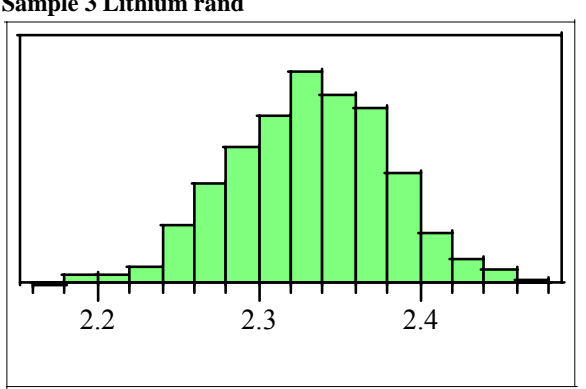

Moments

Mean

Std Dev

upper 95\% Mean

upper $95 \%$ Mea

$95 \%$ Mea

1000
Sample 3 Magnesium rand

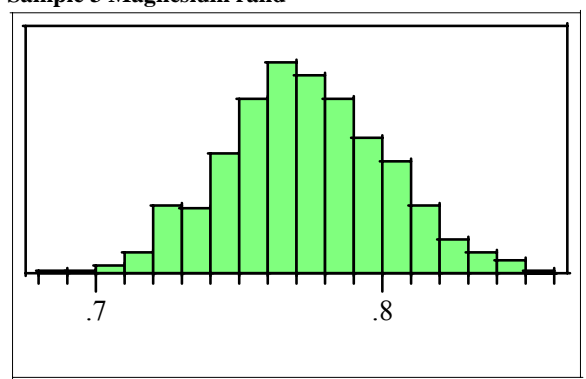

Moments

Mean

Std Err Mean

upper $95 \%$ Mean

lower $95 \%$ Mean

0.7730272

0.028265

0.0008938

0.7747812

1000

Sample 3 Manganese rand

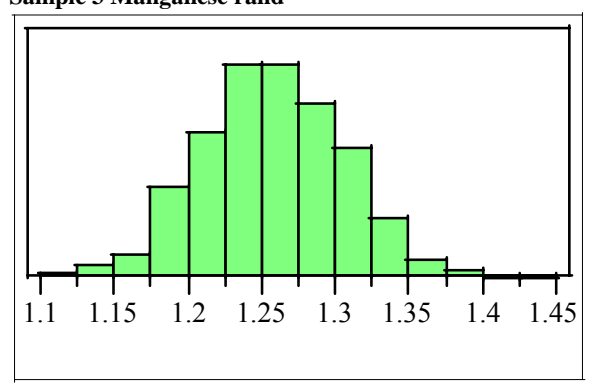

Moments

Mean

Std Err Mean

upper 95\% Mean

lower 95\% Mea

0.0015169
1.2607116

1.2607116

1000 


\section{Exhibit A9. Uncertainties of Inputs to WL Estimation}

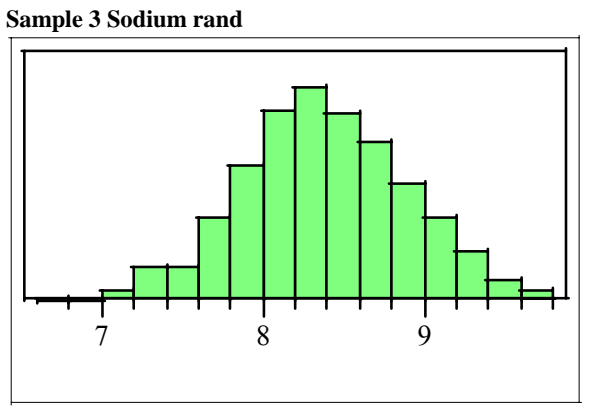

Moments

Mean

Std Dev

Std $95 \%$ Me

lower $95 \%$ Mean

8.3816208

0.5051904

8.4129702

Sample 3 Nickel rand

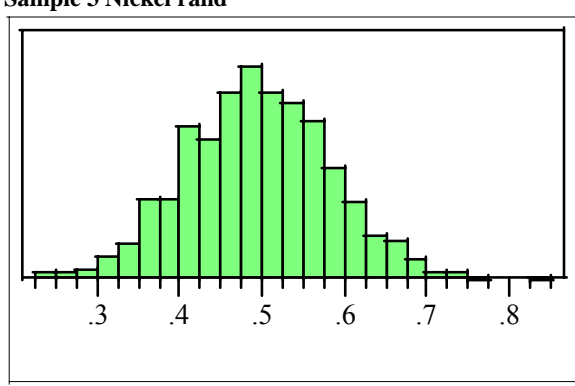

Moments

Mean

Std Dev

lower $95 \%$ Mean

0.4948066

0.0871556

0.0027561

0.4893982

1000
Sample 3 Silicon rand

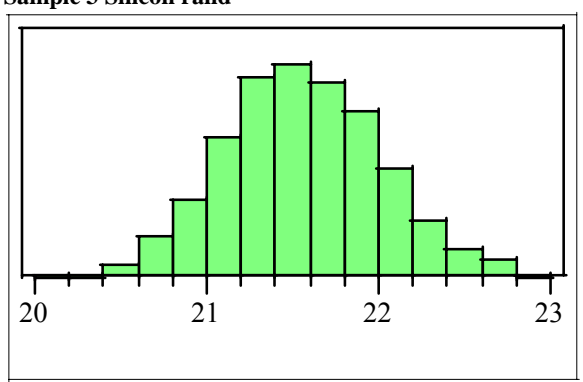

Moments

Mean

Std Dev

10 $95 \%$ Men

upper $95 \%$ Mea

21.56003

0.4570451

0.014453

21.588392

1000

Sample 3 Titanium rand

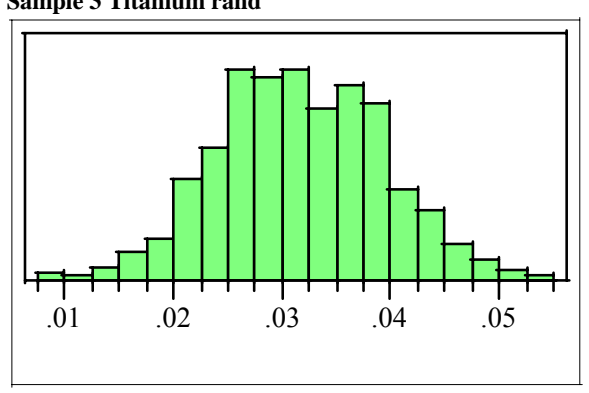

Moments

Mean

Std Err Mean

1

upper $95 \%$ Mea

$95 \%$ Mean
0.0316716

0.007832

0.0002477

0.0321576

1056
Sample 3 Uranium rand

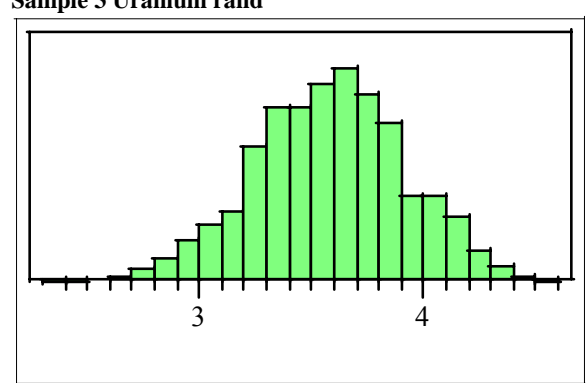

Moments

Mean

Std Err Mean

upper $95 \%$ Mean

lower 95\% Mean

3.5828415

0.3383134

0.0106984

3.6038354

Sample 3 Zirconium rand

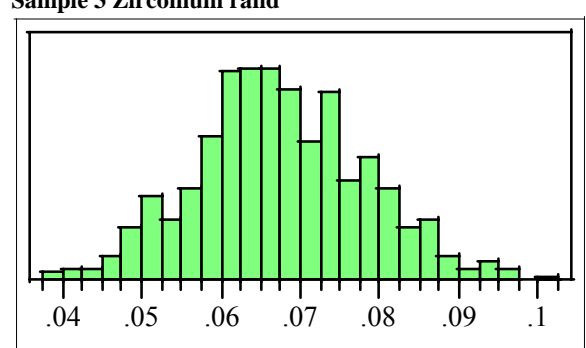

Moments

Mean

0.0675057

Std Err Mea

upper 95\% Mean

lower 95\% Mean $\quad 0.066835$

0.0003415

0.0681759 


\section{Exhibit A9. Uncertainties of Inputs to WL Estimation}

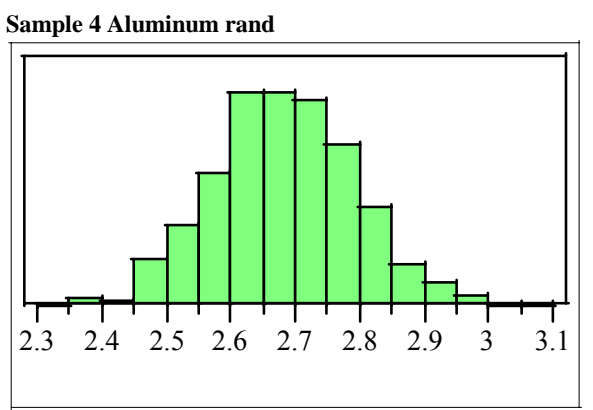

Moments

Mean

Std Dev

Std $95 \%$ Me

lower $95 \%$ Mean

2.6830387

0.1083886

0.0034275

Sample 4 Boron rand

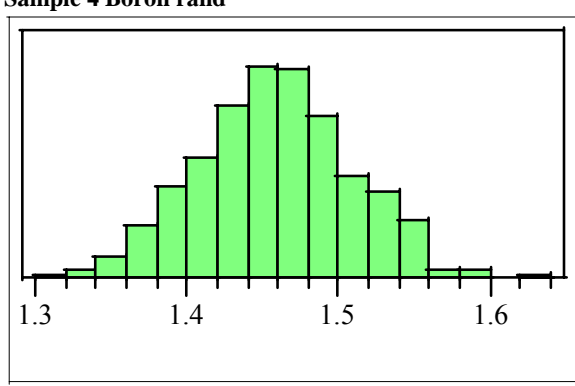

Moments

Mean

Std Dev

lower $95 \%$ Mean

1.4583574

0.0512704

1.4615389

1.4615389

1000

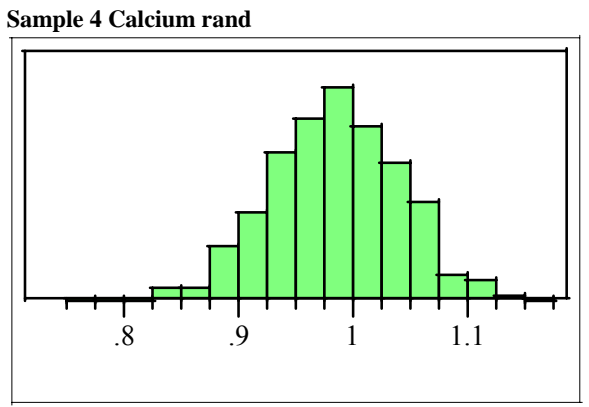

Moments

Mean

Std Err Mean

upper $95 \%$ Mean

upper $95 \%$ Mea

0.9836845

0.0572296

0.0018098

0.9872359

1000

Sample 4 Chromium rand

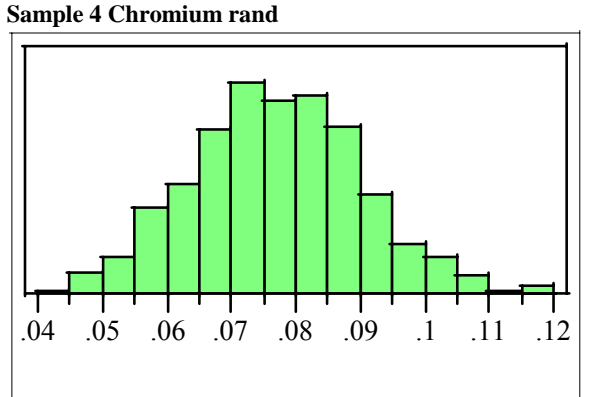

Moments

Mean

Std Dev

1) $95 \%$ Mean

upper $95 \%$ Mea

$\mathrm{N}$
0.077153

0.0132214

0.0004181

0.0779735

1000
Sample 4 Copper rand

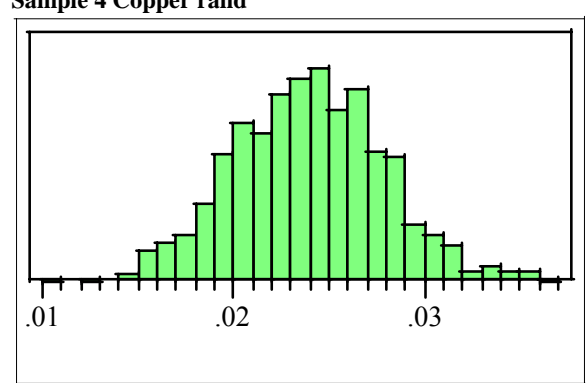

Moments

Mean

Std Err Mean

upper $95 \%$ Mean

lower $95 \%$ Mean

0.0239388

0.0039312

0.000124

0.0241827

Sample 4 Iron rand

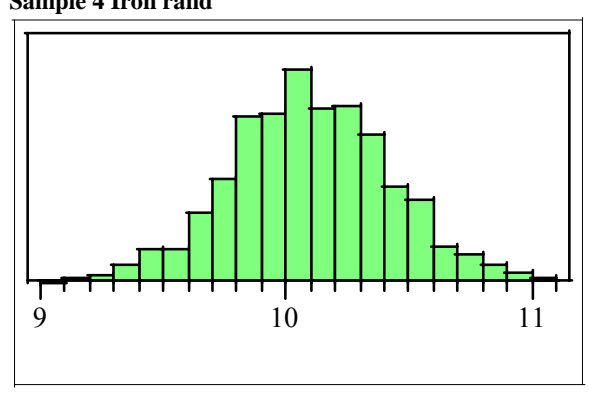

Moments

Mean

10.095789

Std Dev

upper $95 \%$ Mea

lower $95 \%$ Mean $\quad 10.075578$

0.3257001

0.0102995 


\section{Exhibit A9. Uncertainties of Inputs to WL Estimation}

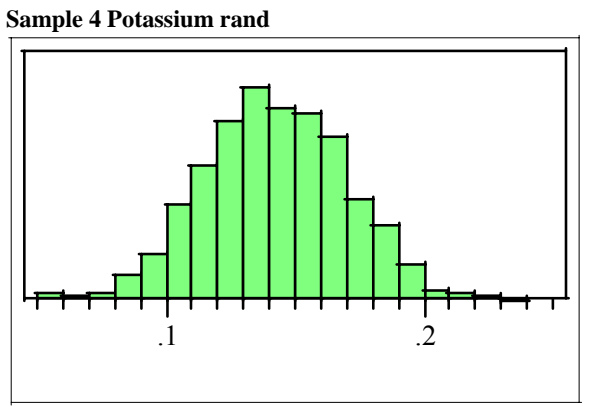

Moments

Mean

Std Dev

upper $95 \%$ Me

upper $95 \%$ Mean

0.1421589

0.0277372

0.1438801

0.1404376

Sample 4 Lithium rand

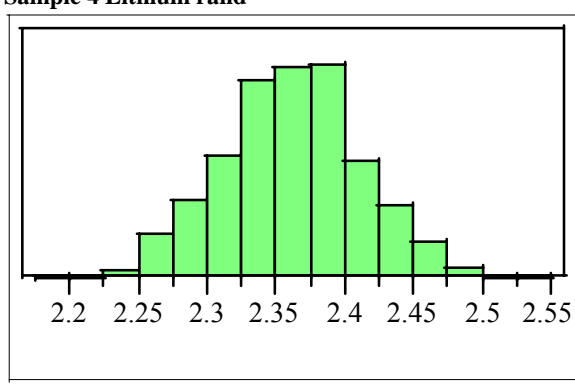

Moments

Mean

Std Dev

upper 95\% Mea

ower 95\% Mean

2.3619907

0.0509036

0.0016097
2.3651495

2.3651495

1000

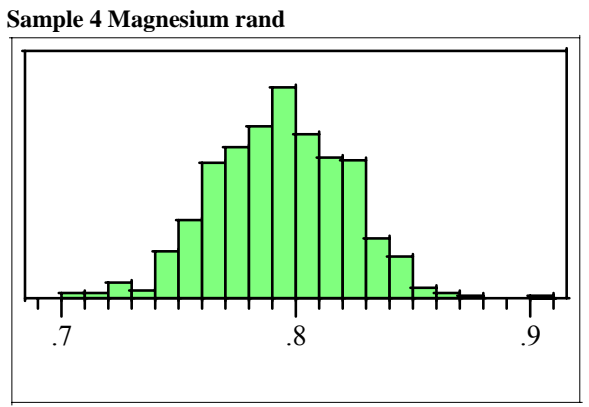

Moments

Mean

Std Err Mean

upper $95 \%$ Mean

lower $95 \%$ Mea

0.7936821

0.0289016

0.0009139

1000

Sample 4 Manganese rand

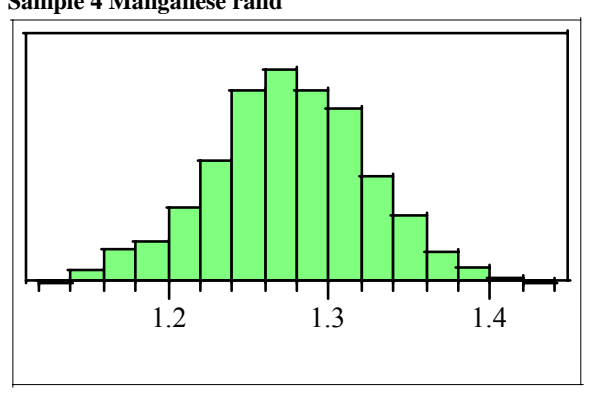

Moments

Mean

Std Dev

upper 95\% Mean

upper $95 \%$ Mea

$5 \%$ Mea

0.0487224
0.0015407

0.0015407
Sample 4 Sodium rand

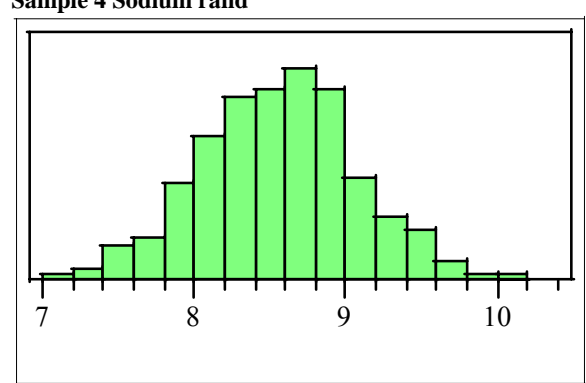

Moments

Mean

Std Err Mean

upper 95\% Mean

0.522425

0.016520

8.5946992

Sample 4 Nickel rand

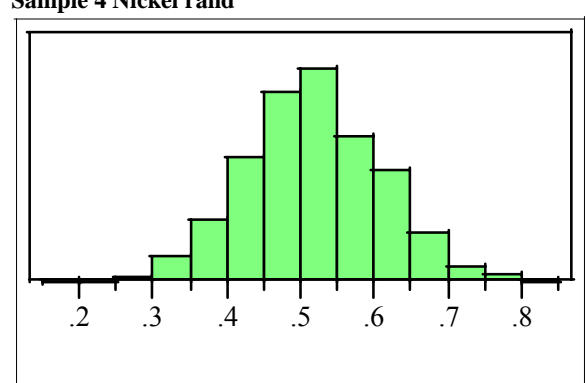

Moments

Mean

0.5165981

Std Dev

upper $95 \%$ Mea

lower $95 \%$ Mea

0.0926662

$\mathrm{N}$

0.0029304

0.5223485
0.5108477

1000 


\section{Exhibit A9. Uncertainties of Inputs to WL Estimation}

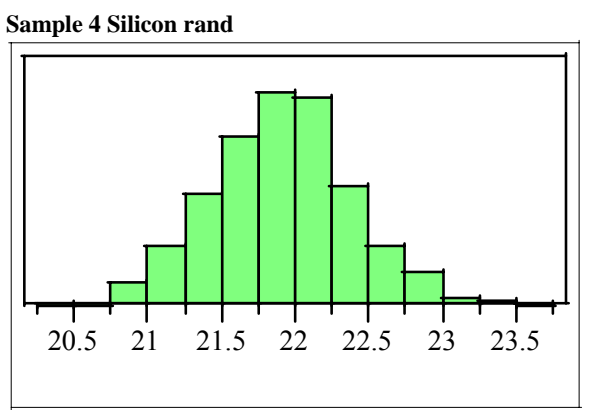

Moments

Mean

Std Dev

Std $95 \%$ Mean

lower $95 \%$ Mean

21.903404

0.4733596

.014968

21.874029

Sample 4 Titanium rand

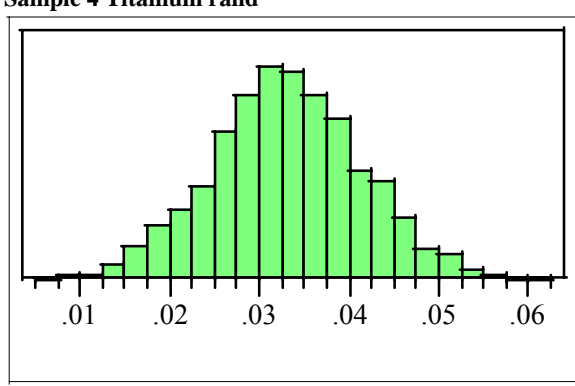

Moments

Mean

Std Dev

upper 95\% Mea

ower 95\% Mean

0.0330058

0.0083819

0.0002651

0.0324856

1000

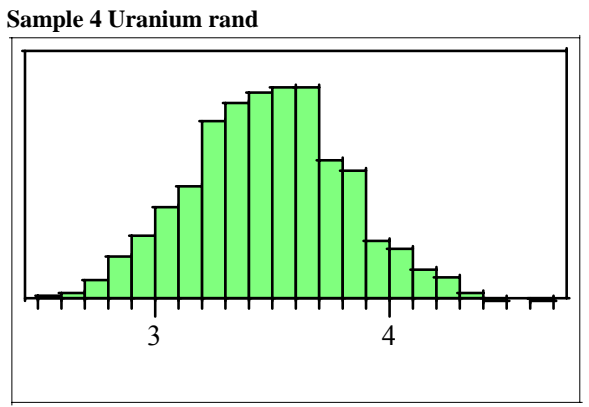

Moments

Mean

Std Dev
Std Err Mean

utd Err Mean

upper $95 \%$ Mea

3.4834769

0.3291783

0.0104095

Sample 4 Zirconium rand

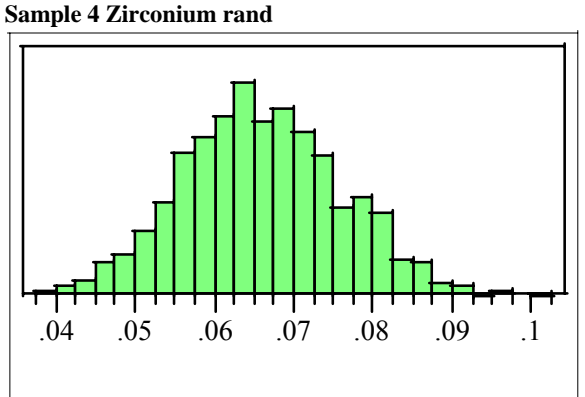

Moments

Mean

Std Dev

upper $95 \%$ Mean

upper $95 \%$ Mea

r $95 \%$ Mean

0.0660073

0.0099542

0.0003148

0.066625

1000
Sample 1 Sum of Oxides rand

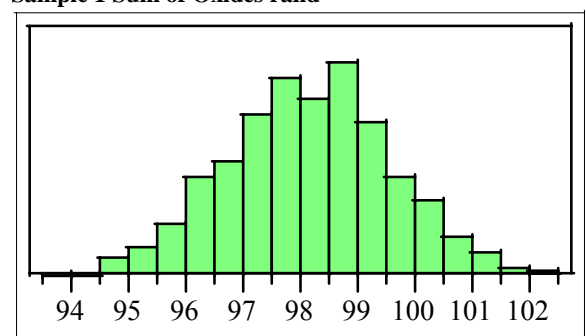

Moments

Mean

Std Err Mean

upper $95 \%$ Mean

lower $95 \%$ Mean

0.045068

0.045068

98.05352

Sample 2 Sum of Oxides rand

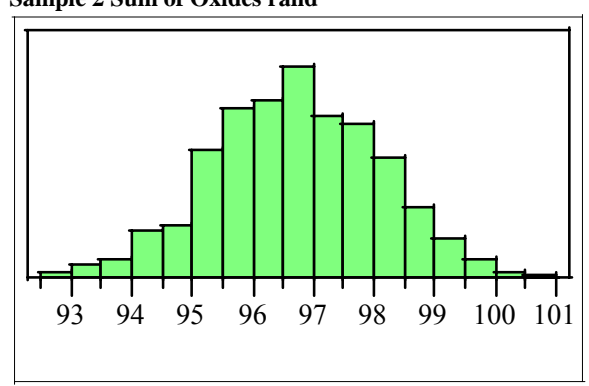

Moments

Mean

96.701304

Std Dev

upper 95\% Mean

lower 95\% Mea

1.391274

0.043996
96.787639

0.0739639
96.614969 
Exhibit A9. Uncertainties of Inputs to WL Estimation

Sample 3 Sum of Oxides rand

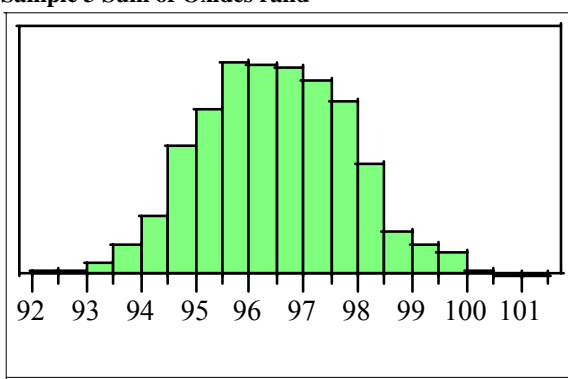

Moments

Mean

Std Err Mean

. $95 \%$ Met

upper $95 \%$ Mea

96.4814

1.3820193

0.0437033

96.567161

Sample 4 Sum of Oxides rand

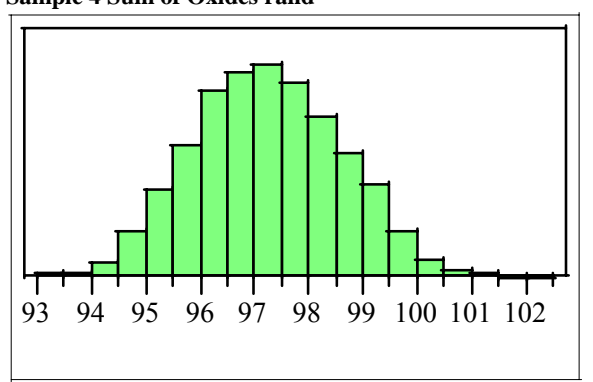

Moments

Mean

Std Dev

upper $95 \%$ Me

lower 95\% Mean

97.247048

1.3767123

0.0435355
97.33248

97.33248
97.161617

1000

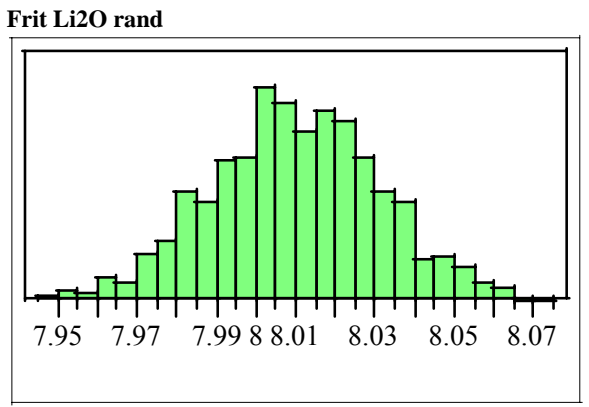

Moments

Mean

Std Err Mean

upper $95 \%$ Mean

lower $95 \%$ Mean

8.0098204

0.0210682

0.0006662

8.0111278

$$
1000
$$


WSRC-TR-2004-00508

Exhibit A10. Relationships between $\mathrm{Li}_{2} \mathrm{O}$ Content and Sum of Oxides of SME Samples
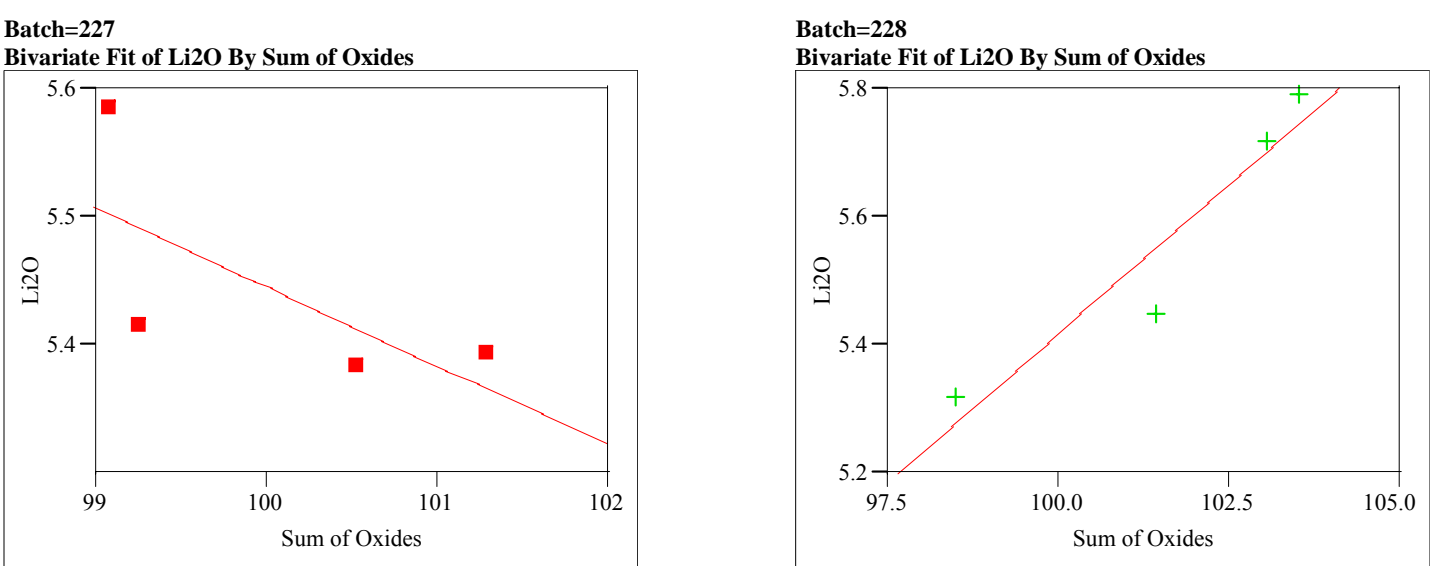

Batch $=229$

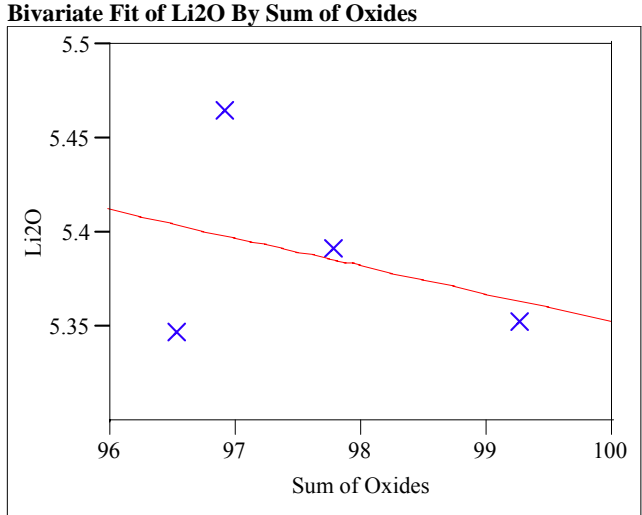

\section{Linear Fit}

Linear Fit

$\mathrm{Li} 2 \mathrm{O}=11.598166-0.0615289$ Sum of Oxides

\section{Linear Fit}

Linear Fit

$\mathrm{Li} 2 \mathrm{O}=-3.914883+0.093324$ Sum of Oxides

\section{Summary of Fit}

\section{Summary of Fit}

$\begin{array}{lr}\text { RSquare } & 0.469648 \\ \text { RSquare Adj } & 0.204473 \\ \text { Root Mean Square Error } & 0.0847 \\ \text { Mean of Response } & 5.443608 \\ \text { Observations (or Sum Wgts) } & 4\end{array}$

\begin{tabular}{lr} 
Observations (or Sum Wgts) & 5.443608 \\
\hline
\end{tabular}

\begin{tabular}{lrrrr}
\multicolumn{9}{l}{ Analysis of Variance } \\
Source & DF & Sum of Squares & Mean Square & F Ratio \\
Model & 1 & 0.01270604 & 0.012706 & 1.7711 \\
Error & 2 & 0.01434833 & 0.007174 & Prob $>$ F \\
C. Total & 3 & 0.02705437 & & 0.3147
\end{tabular}

$\begin{array}{lllll}\text { C. Total } & 3 & 0.02705437 & 0.007174 & \text { Prob }>\text { F }\end{array}$

Parameter Estimates Estimate Std Error t Ratio Prob $>1$

$\begin{array}{lrrrr}\text { Term } & \text { Estimate } & \text { Std Error } & \text { t Ratio } & \text { Prob }>|t| \\ \text { Intercept } & 11.598166 & 4.624828 & 2.51 & 0.1290\end{array}$

$\begin{array}{lrrrr}\text { Intercept } & 11.598166 & 4.624828 & 2.51 & 0.1290 \\ \text { Sum of Oxides } & -0.061529 & 0.046234 & -1.33 & 0.3147\end{array}$

Revision 0

\section{Linear Fit \\ Linear Fit \\ $\mathrm{Li} 2 \mathrm{O}=6.844091-0.0149152$ Sum of Oxides}

Summary of Fit

RSquare

RSquare Adj $\quad 0.111071$

Root Mean Square Error $\quad-0.33339$

Mean of Response 5.38817

5.38817

Analysis of Variance

Source DF Sum of Squares Mean Square F Ratio

$\begin{array}{lllll}\text { Model } \quad 1 & 0.00098573 & 0.000986 & 0.2499\end{array}$

$\begin{array}{lllll}\text { Error } & 2 & 0.00788909 & 0.003945 & \text { Prob }>\text { F } \\ \text { C. Total } & 3 & 0.00887482 & & 0.6667\end{array}$

Parameter Estimates

$\begin{array}{llll}\text { Parameter Estimates } & & & \\ \text { Term } & \text { Estimate Std Error } & t \text { Ratio } & \text { Prob }>|t|\end{array}$

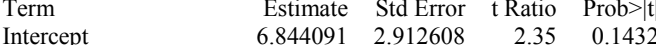

$\begin{array}{lllll}\text { Sum of Oxides } & -0.014915 & 0.029836 & -0.50 & 0.6667\end{array}$ 
WSRC-TR-2004-00508

Exhibit A10. Relationships between $\mathrm{Li}_{2} \mathrm{O}$ Content and Sum of Oxides of SME Samples

Revision 0

Batch $=230$

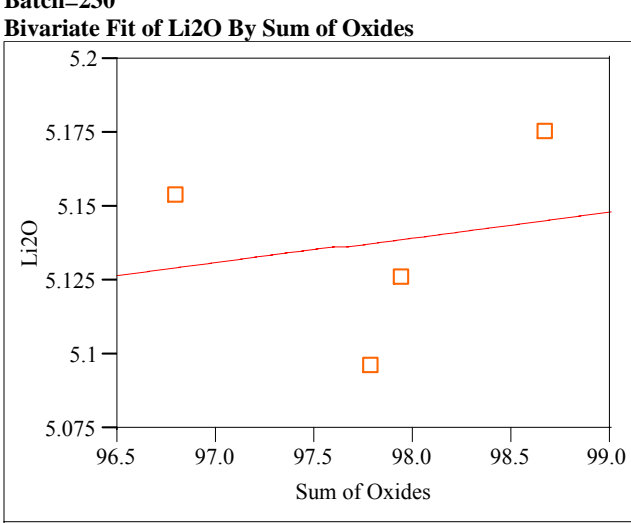

Linear Fit

Linear Fit

$\mathrm{Li} 2 \mathrm{O}=4.2871812+0.0086987$ Sum of Oxides

\section{Summary of Fit}

RSquare

Root Mean Square Error $\quad 0.041517$

Mean of Response

5.137896

Observations (or Sum Wgts)

\section{Analysis of Variance}

Source DF Sum of Squares Mean Square F Ratio

$\begin{array}{lllll}\text { Model } & 1 & 0.00013560 & 0.000136 & 0.0787 \\ \text { Error } & 2 & 0.00344724 & 0.001724 & \text { Plob }>\text { F }\end{array}$

$\begin{array}{lllrr}\text { Error } & 2 & 0.00344724 & 0.001724 & \text { Prob }>\text { F } \\ \text { C. Total } & 3 & 0.00358284 & & 0.8055\end{array}$

Parameter Estimates

Term

Estimate Std Error $t$ Ratio Prob $>|t|$

$\begin{array}{lrrrr} & 4.2871812 & 3.03312 & 1.41 & 0.2931 \\ \text { Sum of Oxides } & 0.0086987 & 0.031013 & 0.28 & 0.8055\end{array}$
Batch=231

Bivariate Fit of Li2O By Sum of Oxides

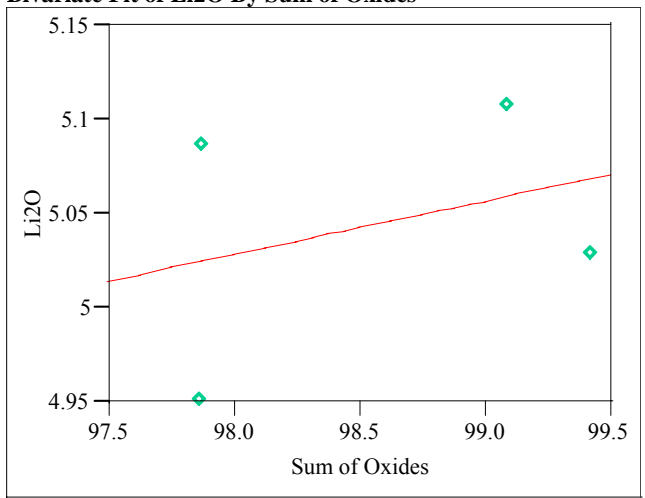

\section{Linear Fit}

Linear Fit

$\mathrm{Li} 2 \mathrm{O}=2.2605519+0.0282428$ Sum of Oxides

Summary of Fit

$\begin{array}{ll}\text { RSquare } & 0.106645 \\ \text { RSquare Adj } & -0.34003 \\ \text { Root Mean Square Error } & 0.081369 \\ \text { Mean of Response } & 5.044245\end{array}$

Observations (or Sum Wgts)

Analysis of Variance

Source DF Sum of Squares Mean Square F Ratio

$\begin{array}{llllll}\text { Model } & 1 & 0.00158076 & 0.001581 & 0.2388\end{array}$

$\begin{array}{lllrr}\text { Error } & 2 & 0.01324190 & 0.006621 & \text { Prob }>\text { F } \\ \text { C. Total } & 3 & 0.01482266 & & 0.6734\end{array}$

Parameter Estimates

$\begin{array}{lrlll}\text { Parameter Estimates } & \text { Estimate } & \text { Std Error } & \mathrm{t} \text { Ratio } & \text { Prob }>|\mathrm{t}|\end{array}$

$\begin{array}{lllll}\text { Intercept } & 2.2605519 & 5.697181 & 0.40 & 0.7299\end{array}$
Batch $=232$

Bivariate Fit of Li2O By Sum of Oxides

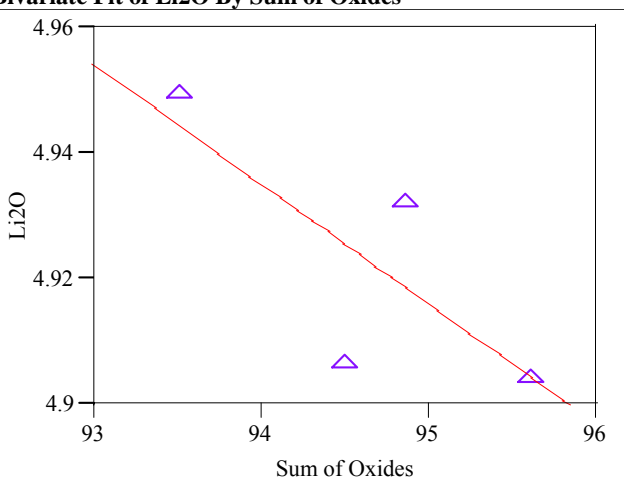

\section{Linear Fit}

Linear Fit

$\mathrm{Li} 2 \mathrm{O}=6.7293915-0.019088$ Sum of Oxides

Summary of Fit

$\begin{array}{ll}\text { RSquare } & 0.59196 \\ \text { RSquare Adj } & 0.38794\end{array}$

RSquare Adj $\quad 0.387941$

Root Mean Square Enor $\quad 0.016976$

Mean of Response $\quad 4.92314$

Analysis of Variance

Source DF Sum of Squares Mean Square F Ratio

$\begin{array}{lllll}\text { Model } & 1 & 0.00083615 & 0.000836 & 2.9015\end{array}$

$\begin{array}{lllll}\text { Error } & 2 & 0.00057636 & 0.000288 & \text { Prob }>\text { F }\end{array}$

Parameter Estimates

$\begin{array}{lrrrr}\text { Parameter Estimates } & & & & \\ \text { Term } & \text { Estimate } & \text { Std Error } & \text { t Ratio } & \text { Prob }>|t| \\ \text { Intercept } & 6.7293915 & 1.060427 & 6.35 & 0.0239\end{array}$

$\begin{array}{lllllll} & \text { Sum of Oxides } & -0.019088 & 0.011206 & -1.70 & 0.2306\end{array}$ 
WSRC-TR-2004-00508

Exhibit A10. Relationships between $\mathrm{Li}_{2} \mathrm{O}$ Content and Sum of Oxides of SME Samples

Revision 0

Batch=233

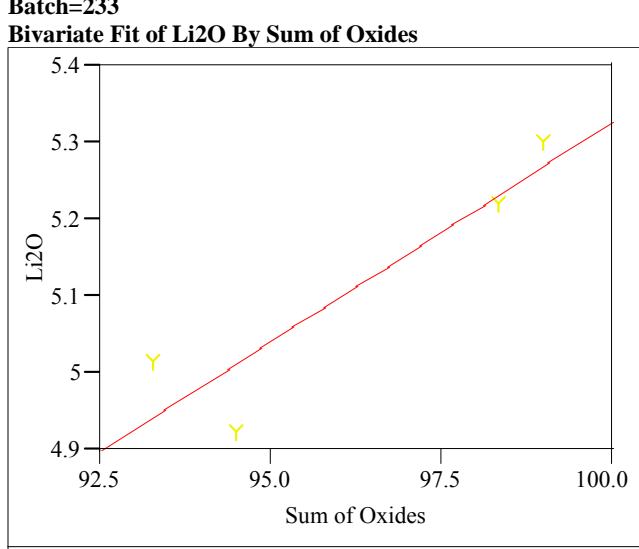

\section{Linear Fit}

Linear Fit

$\mathrm{Li} 2 \mathrm{O}=-0.385165+0.0571142$ Sum of Oxide

\section{Summary of Fit}

RSquare

RSquare Adj

0.843759

Root Mean Sq

Mean of Response

Th
4

\section{Analysis of Variance}

Source DF Sum of Squares Mean Square F Ratio

$\begin{array}{lllll}\text { Model } & 1 & 0.07756686 & 0.077567 & 10.8007\end{array}$

$\begin{array}{lllll}\text { Error } & 2 & 0.01436331 & 0.007182 & \text { Prob }>\text { F }\end{array}$

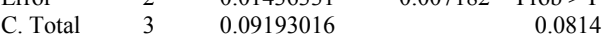

\section{Parameter Estimates}

Term

$\begin{array}{rrrr}\text { Estimate } & \text { Std Error } & \text { t Ratio } & \text { Prob }>|t| \\ -0.385165 & 1.674218 & -0.23 & 0.8394\end{array}$

$\begin{array}{lrrrr}\text { Intercept } & -0.385165 & 1.674218 & -0.23 & 0.8394 \\ \text { Sum of Oxides } & 0.0571142 & 0.017379 & 3.29 & 0.0814\end{array}$
Batch $=234$

Bivariate Fit of Li2O By Sum of Oxides

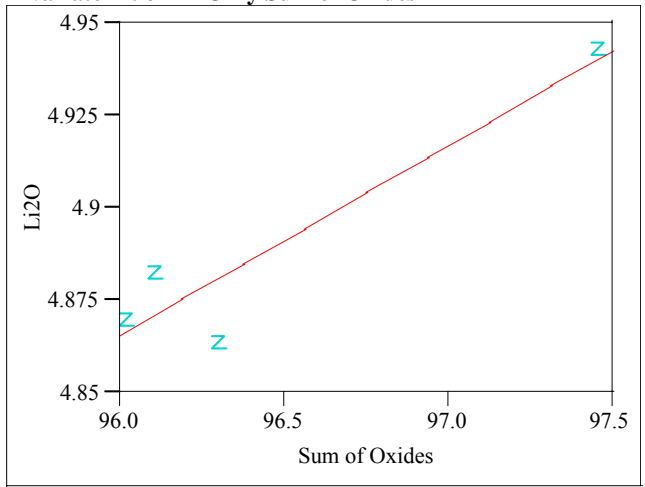

\section{Linear Fit}

Linear Fit

$\mathrm{Li} 2 \mathrm{O}=-0.059662+0.0513035$ Sum of Oxides

Summary of Fit

$\begin{array}{lr}\text { RSquare } & 0.882189 \\ \text { RSquare Adj } & 0.823283 \\ \text { Root Mean Square Error } & 0.015312 \\ \text { Mean of Response } & 4.889774 \\ \text { Observations (or Sum Wgts) } & 4\end{array}$

Observations (or Sum Wgts)

Analysis of Variance

Source DF Sum of Squares Mean Square F Ratio

$\begin{array}{lllll}\text { Model } & 1 & 0.00351137 & 0.003511 & 14.9763\end{array}$

$\begin{array}{lllrr}\text { Error } & 2 & 0.00046892 & 0.000234 & \text { Prob }>\text { F } \\ \text { C. Total } & 3 & 0.00398029 & & 0.0608\end{array}$

Parameter Estimates

Term Estimate Std Error t Ratio Prob $>|t|$

Intercept

$\begin{array}{rrrr}\text { Estimate } & \text { Std Error } & \text { t Ratio } & \text { Prob }>|t| \\ -0.059662 & 1.278971 & -0.05 & 0.9670\end{array}$

$\begin{array}{llllll}\text { Sum of Oxides } & 0.0513035 & 0.013257 & 3.87 & 0.0608\end{array}$
Batch $=235$

Bichite Fit of Li2O By Sum of Oxides

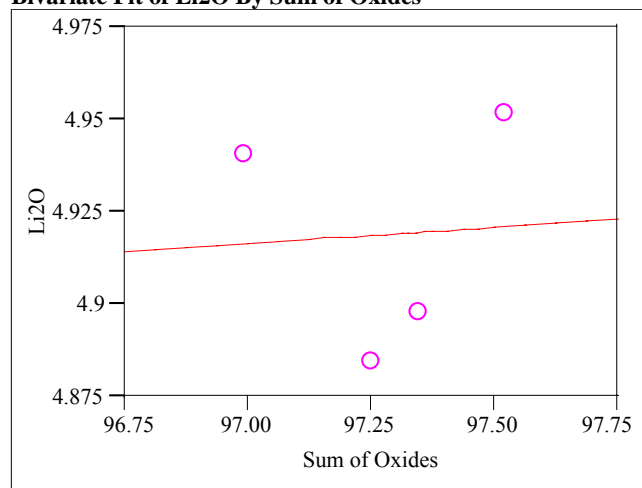

\section{Linear Fit}

Linear Fit

$\mathrm{Li} 2 \mathrm{O}=4.0977761+0.0084403$ Sum of Oxides

Summary of Fit

$\begin{array}{lr}\text { RSquare } & 0.003311 \\ \text { RSquare Adj } & -0.49503 \\ \text { Root Mean Square Error } & 0.039654 \\ \text { Mean of Response } & 4.918838 \\ \text { Observations (or Sum Wgts) } & 4\end{array}$

$\begin{array}{llll}\text { Analysis of Variance } & \\ \text { Source DF Sum of Squares Mean Square F Ratio }\end{array}$

$\begin{array}{lrrrr}\text { Model } & 1 & 0.00001045 & 0.000010 & 0.0066\end{array}$

$\begin{array}{lllll}\text { Error } & 2 & 0.00314481 & 0.001572 & \text { Prob }>\text { F }\end{array}$

$\begin{array}{llll}\text { C. Total } & 3 & 0.00315526 & 0.001525\end{array}$

Parameter Estimates

Term Estimate Std Error $t$ Ratio Prob $>|t|$

$\begin{array}{lllll}\text { Intercept } & 4.0977761 & 10.0724 & 0.41 & 0.7235\end{array}$

$\begin{array}{llllll}\text { Sum of Oxides } & 0.0084403 & 0.103541 & 0.08 & 0.9425\end{array}$ 
WSRC-TR-2004-00508

Exhibit A10. Relationships between $\mathrm{Li}_{2} \mathrm{O}$ Content and Sum of Oxides of SME Samples

Revision 0

Batch $=236$

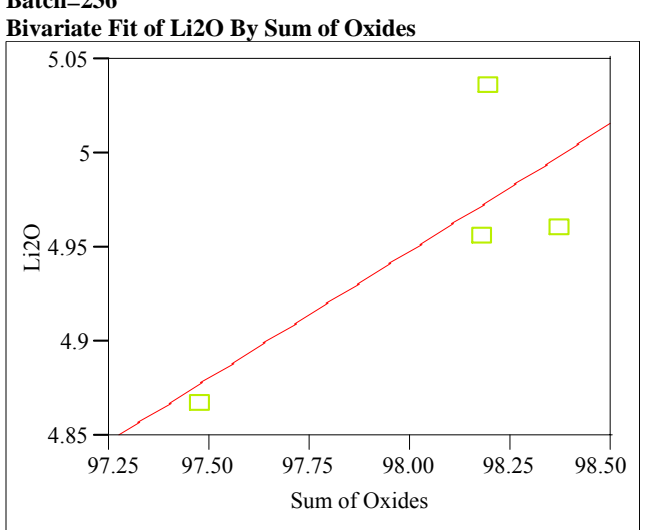

\section{Linear Fit}

Linear Fit

$\mathrm{Li} 2 \mathrm{O}=-8.316597+0.135352$ Sum of Oxides

\section{Summary of Fit}

$\begin{array}{ll}\text { RSquare } & 0.608249 \\ \text { RSquare Adj } & 0.412373 \\ \text { Root Mean Square Error } & 0.052647 \\ \text { Mean of Response } & 4.954899\end{array}$

Mean of Response $\quad 4.954899$

Observations (or Sum Wgts)

\begin{tabular}{|c|c|c|c|c|}
\hline \multicolumn{5}{|c|}{ f Variance } \\
\hline Source & DF & Sum of Squares & Mean Square & F Ratio \\
\hline Model & 1 & 0.00860708 & 0.008607 & 3.1053 \\
\hline Error & 2 & 0.00554351 & 0.002772 & Prob $>F$ \\
\hline C. Total & 3 & 0.01415059 & & 0.2201 \\
\hline
\end{tabular}

$\begin{array}{lllll}\text { Error } & 2 & 0.00554351 & 0.002772 & \text { Prob }>\text { F } \\ \text { C. Total } & 3 & 0.01415059 & & 0.2201\end{array}$

Parameter Estimates

Term Estimate Std Error $\quad$ t Ratio Prob $>|\mathrm{t}|$

$\begin{array}{lllll}\text { Intercept } & -8.316597 & 7.531335 & -1.10 & 0.3846\end{array}$
Batch $=237$

Bivariate Fit of Li2O By Sum of Oxides

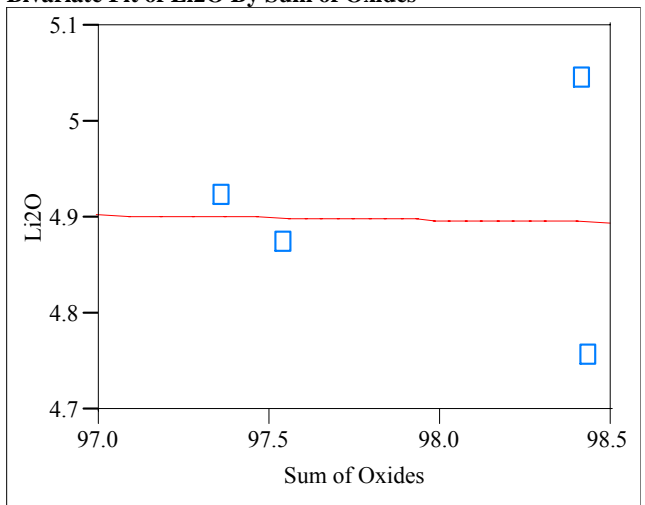

\section{Linear Fit}

Linear Fit

Linear Fit
Li2O $=5.3739087-0.0048608$ Sum of Oxides

Summary of Fit

RSquare

RSquare Adj

Root Mean Square Er

Mean of Response

0.000525

$-0.49921$

0.147384

4.897848

Analysis of Variance

Source DF Sum of Squares Mean Square F Ratio

$\begin{array}{lllll}\text { Model } & 1 & 0.00002280 & 0.000023 & 0.0010\end{array}$

$\begin{array}{lllll}\text { Error } & 2 & 0.04344403 & 0.021722 & \text { Prob }>\text { F } \\ \text { C. To } & 3 & 0.0434683 & & 0.977\end{array}$

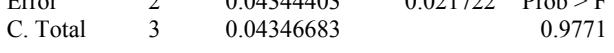

\section{Parameter Estimates}

Term Estimate Std Error $t$ Ratio Prob $>\mid t$

$\begin{array}{lrrrr}\text { Intercept } & 5.3739087 & 14.69399 & 0.37 & 0.7496\end{array}$

$\begin{array}{lrrrr}\text { Sum of Oxides } & 5.3739087 & 14.69399 & 0.37 & 0.7496 \\ & -0.004861 & 0.15003 & -0.03 & 0.9771\end{array}$
Batch $=238$

Bivariate Fit of Li2O By Sum of Oxides

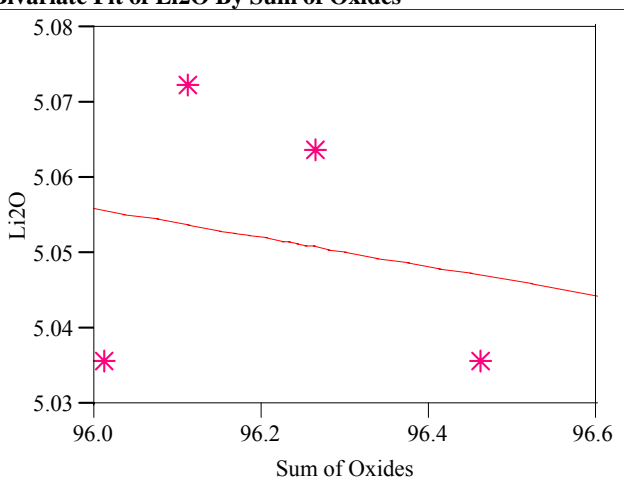

\section{Linear Fit}

Linear Fit

$\mathrm{Li} 2 \mathrm{O}=6.8897496-0.0191028$ Sum of Oxides

Summary of Fit

RSquare

RSquare Adj

Root Mean Square Error $\quad-0.44171$

$\begin{array}{lr}\text { Mean of Response } & 5.05178\end{array}$

4

Analysis of Variance

Source DF Sum of Squares Mean Square F Ratio

$\begin{array}{lllll}\text { Model } & 1 & 0.00004197 & 0.000042 & 0.0809 \\ \text { Eror } & 2 & 0.00103798 & 0.000519 & 0.8029\end{array}$

$\begin{array}{llllr}\text { Error } & 2 & 0.00103798 & 0.000519 & \text { Prob }>\text { F } \\ \text { C. Total } & 3 & 0.00107995 & & 0.8029\end{array}$

Parameter Estimates

$\begin{array}{lrlrr}\text { Parameter Estimates } & & & & \\ \text { Term } & \text { Estimate } & \text { Std Error } & \mathrm{t} \text { Ratio } & \text { Prob }>|t| \\ \text { Intercept } & 6.8897496 & 6.463227 & 1.07 & 0.3981\end{array}$

$\begin{array}{lllll}\text { Sum of Oxides } & -0.019103 & 0.067175 & -0.28 & 0.8029\end{array}$ 
WSRC-TR-2004-00508

Exhibit A10. Relationships between $\mathrm{Li}_{2} \mathrm{O}$ Content and Sum of Oxides of SME Samples

Revision 0

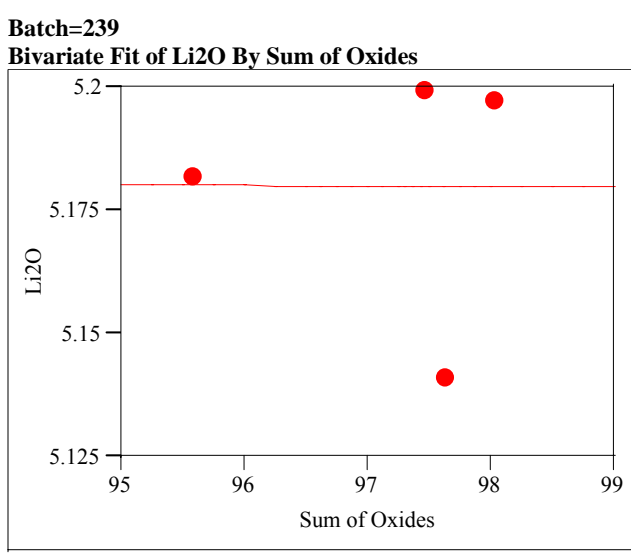

Linear Fit

Li2O $=5.1921022-0.0001258$ Sum of Oxides

\section{Summary of Fit}

$\begin{array}{lr}\text { RSquare } & 0.000026 \\ \text { RSquare Adj } & -0.49996 \\ \text { Root Mean Square Error } & 0.033003 \\ \text { Mean of Response } & 5.179877 \\ \text { Observations (or Sum Wgts) } & 4\end{array}$

\section{Analysis of Variance}

Source DF Sum of Squares Mean Square F Ratio

$\begin{array}{lllll}\text { Model } & 1 & 0.00000006 & 0.000000 & 0.0001 \\ \text { Error } & 2 & 0.00217838 & 0.001089 & \text { Pro }\end{array}$

$\begin{array}{lllrr}\text { C. Total } & 3 & 0.00217838 & 0.001089 & \text { Prob }>\text { F } \\ \text { Cror } & 3 & 0.00217844 & & 0.9949\end{array}$

Parameter Estimates

Term

Estimate Std Error $\mathrm{t}$ Ratio Prob $>|\mathrm{t}|$

$\begin{array}{lrrrr} & 5.1921022 & 1.692281 & 3.07 & 0.0918 \\ \text { Sum of Oxides } & -0.000126 & 0.017412 & -0.01 & 0.9949\end{array}$
Batch $=240$

Bivariate Fit of Li20 By Sum of Oxides

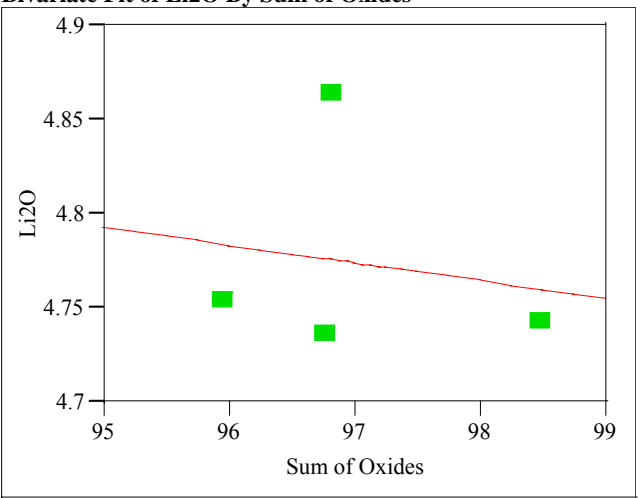

\section{Linear Fit}

Linear Fit

$\mathrm{Li2O}=5.6847488-0.0093903$ Sum of Oxides

Summary of Fit

RSquare

RSquare Adj

Rquare E

0.027753

$-0.45837$

4.774056

Observations (or Sum Wgts)

4

Analysis of Variance

Source DF Sum of Squares Mean Square F Ratio

Model $\quad 1 \quad 0.00029959$

$\begin{array}{lllrr}\text { Error } & 2 & 0.01049527 & 0.005248 & \text { Prob }>\text { F } \\ \text { C. Total } & 3 & 0.01079486 & & 0.8334\end{array}$

Parameter Estimates

Term Estimate Std Error t Ratio Prob $>|t|$

$\begin{array}{llrrr}\text { Intercept } & 5.6847488 & 3.811591 & 1.49 & 0.2744\end{array}$
Batch $=241$

Bivariate Fit of Li2O By Sum of Oxides

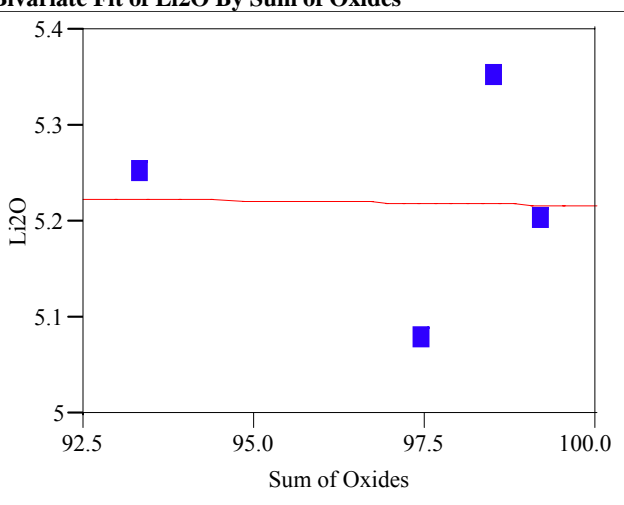

Linear Fit

Linear Fit

$\mathrm{Li} 2 \mathrm{O}=5.3172252-0.001004$ Sum of Oxides

Summary of Fit

RSquare

RSquare Adj $\quad \begin{array}{ll}0.000544 \\ -0.49918\end{array}$

Root Mean Square Error $\quad 0.139195$

$\begin{array}{lr}\text { Mean of Response } & 5.219706 \\ \text { Observations (or Sum Wgts) } & 4\end{array}$

Analysis of Varianc

Source DF Sum of Squares Mean Square F Ratio

$\begin{array}{lllll}\text { Model } & 1 & 0.00002109 & 0.000021 & 0.0011\end{array}$

$\begin{array}{lllll}\text { Error } & 2 & 0.03875050 & 0.019375 & \text { Prob }>\text { F }\end{array}$

$\begin{array}{llll}\text { C. Total } & 3 & 0.03877159 & 0.9767\end{array}$

Parameter Estimates

$\begin{array}{lrrr}\text { Term Estimate } & \text { Std Error t Ratio Prob }>|t|\end{array}$

$\begin{array}{lllll}\text { Intercept } & 5.3172252 & 2.956356 & 1.80 & 0.2139\end{array}$

$\begin{array}{lllll}\text { Sum of Oxides } & -0.001004 & 0.030429 & -0.03 & 0.9767\end{array}$ 
WSRC-TR-2004-00508

Exhibit A10. Relationships between $\mathrm{Li}_{2} \mathrm{O}$ Content and Sum of Oxides of SME Samples

Revision 0

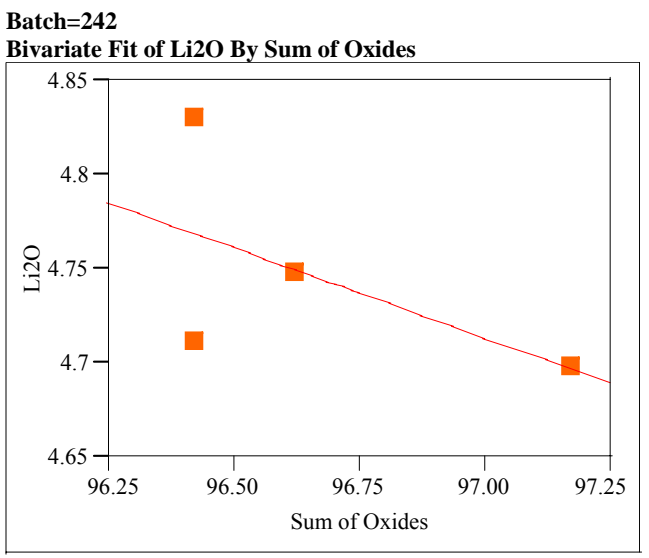

\section{Linear Fit}

Linear Fit

$\mathrm{Li} 2 \mathrm{O}=14.008491-0.0958273$ Sum of Oxides

\section{Summary of Fit}

$\begin{array}{ll}\text { RSquare } & 0.330238 \\ \text { RSquare Adj } & -0.00464 \\ \text { Root Mean Square Error } & 0.059241 \\ \text { Mean of Response } & 4.746068\end{array}$

Observations (or Sum Wgts)

Batch $=243$

Bivarite Fit of Li2O By Sum of Oxides

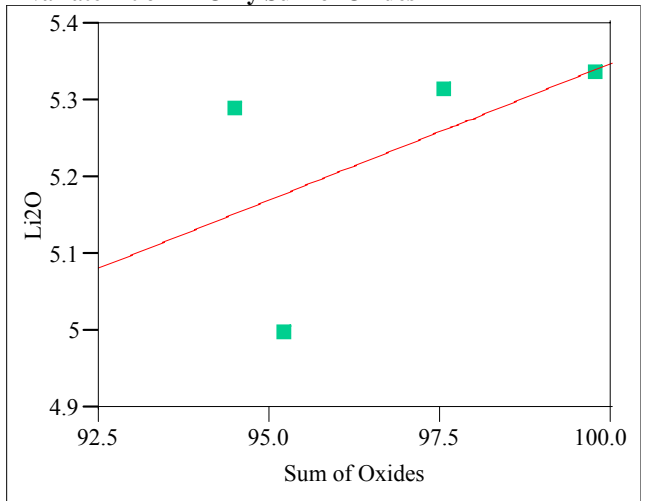

\section{Linear Fit}

Linear Fit

$\mathrm{Li} 2 \mathrm{O}=1.791165+0.0355723$ Sum of Oxide

Summary of Fit

RSquare

RSquare Adj

Root Mean Square Erro

$\begin{array}{lr}\text { Mean of Response } & 5.232623 \\ \text { Observations (or Sum Wgts) } & 4\end{array}$

$-0.07216$

0.165438

Analysis of Variance

Source DF Sum of Squares Mean Square F Ratio

$\begin{array}{lllll}\text { Model } & 1 & 0.00346079 & 0.003461 & 0.9861\end{array}$

$\begin{array}{lllll}\text { Error } & 2 & 0.00701889 & 0.003509 & \text { Prob }>\text { F }\end{array}$

Parameter Estimates

$\begin{array}{lrrrr}\text { Parameter Estimates } & & & & \\ \text { Term } & \text { Estimate } & \text { Std Error } & \mathrm{t} \text { Ratio } & \text { Prob }>|\mathrm{t}| \\ \text { Intercept } & 14.008491 & 9.327354 & 1.50 & 0.2720\end{array}$

\section{Analysis of Variance}

Source DF Sum of Squares Mean Square F Ratio

$\begin{array}{lllll}\text { Model } & 1 & 0.02184398 & 0.021844 & 0.7981\end{array}$

$\begin{array}{llllll}\text { Error } & 2 & 0.05473977 & 0.027370 & \text { Prob }>\text { F }\end{array}$

$\begin{array}{llll}\text { C. Total } & 3 & 0.07658375 & 0.4659\end{array}$

\section{Parameter Estimates}

Term Estimate Std Error $t$ Ratio Prob $>\mid t$

$\begin{array}{lllll}\text { Intercept } & 1.791165 & 3.853126 & 0.46 & 0.6877\end{array}$

$\begin{array}{lllll}\text { Sum of Oxides } & 0.0355723 & 0.039818 & 0.89 & 0.4659\end{array}$
Batch $=244$

Bivariate Fit of Li2O By Sum of Oxides

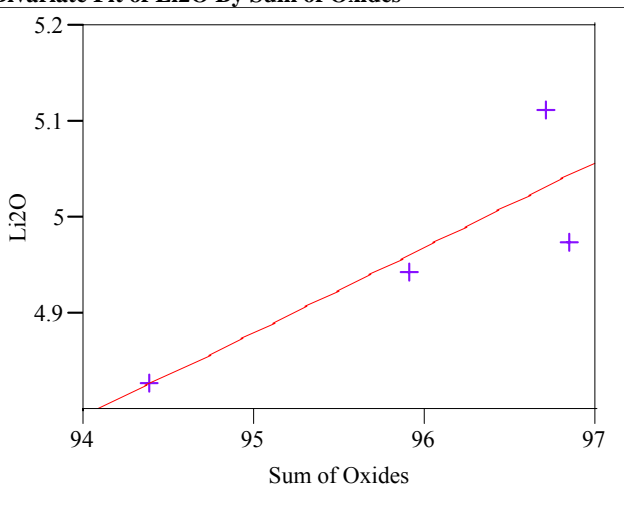

\section{Linear Fit}

Linear Fit

Li2O $=-3.528828+0.0885128$ Sum of Oxides
.

Summary of Fit

RSquare

RSquare Adj $\quad 0.719412$

Root Mean Square Error $\quad 0.076424$

Mean of Response $\quad 4.964587$

Observations (or Sum Wgts) 4

Analysis of Variance

Source DF Sum of Squares Mean Square F Ratio

$\begin{array}{lllll}\text { Model } & 1 & 0.02995010 & 0.029950 & 5.1279\end{array}$

$\begin{array}{lllrr}\text { Error } & 2 & 0.01168128 & 0.005841 & \text { Prob }>\text { F } \\ \text { C. Total } & 3 & 0.04163138 & & 0.1518\end{array}$

Parameter Estimates

Term Estimate Std Error t Ratio Prob $>\mid$

$\begin{array}{lllll}\text { Intercept } & -3.528828 & 3.750905 & -0.94 & 0.446\end{array}$

$\begin{array}{lllll}\text { Sum of Oxides } & 0.0885128 & 0.039087 & 2.26 & 0.1518\end{array}$ 
WSRC-TR-2004-00508

Exhibit A10. Relationships between $\mathrm{Li}_{2} \mathrm{O}$ Content and Sum of Oxides of SME Samples

Revision 0

Batch $=245$

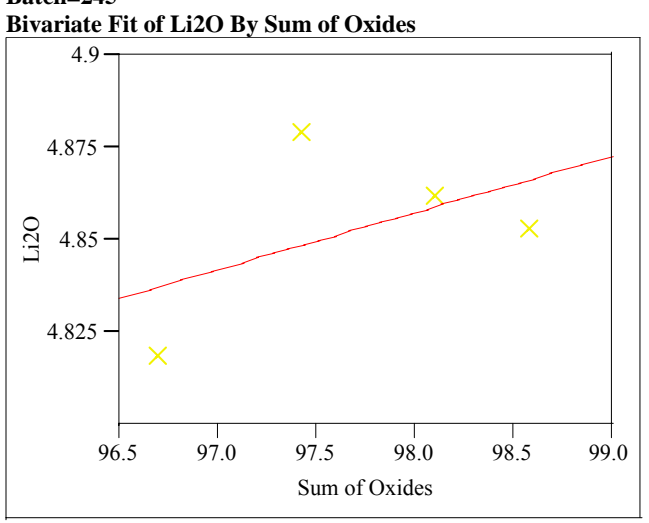

Linear Fit

Linear Fit

$\mathrm{Li2O}=3.3504045+0.0153763$ Sum of Oxides

\section{Summary of Fit}

RSquare

RSquare Adj

Root Mean Square Error

Mean of Response

0.24709

Observations (or Sum Wgts)

$-0.12936$

4.852637

Analysis of Variance

Source DF Sum of Squares Mean Square F Ratio

$\begin{array}{lrrrr}\text { Model } & 1 & 0.00047643 & 0.000476 & 0.6564\end{array}$

$\begin{array}{llrrr}\text { Error } & 2 & 0.00145172 & 0.000726 & \text { Prob }>\text { F } \\ \text { C. Total } & 3 & 0.00192815 & 0.5029\end{array}$

Parameter Estimates

Term

Estimate Std Error $\quad \mathrm{t}$ Ratio Prob $>|\mathrm{t}|$

$\begin{array}{lllrr} & 3.3504045 & 1.854287 & 1.81 & 0.2125 \\ \text { Sum of Oxides } & 0.0153763 & 0.018979 & 0.81 & 0.5029\end{array}$
Batch $=246$

Bivariate Fit of Li2O By Sum of Oxides

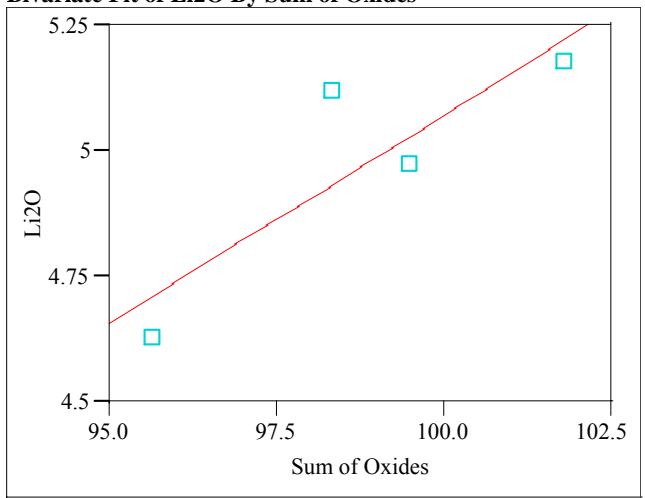

\section{Linear Fit}

Linear Fit

$\mathrm{Li} 2 \mathrm{O}=-3.215316+0.0828688$ Sum of Oxides

Summary of Fit

RSquare Adj

Mean of Response

0.151581

Observations (or Sum Wgts)

4

Analysis of Variance

Source DF Sum of Squares Mean Square F Ratio

$\begin{array}{lllll}\text { Model } & 1 & 0.13534217 & 0.135342 & 5.8904\end{array}$

$\begin{array}{lllll}\text { Error } & 2 & 0.04595385 & 0.022977 & \text { Prob }>\text { F }\end{array}$

Parameter Estimates

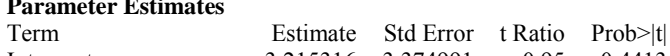
$\begin{array}{lllll}\text { Intercept } & -3.215316 & 3.374991 & -0.95 & 0.4413 \\ \text { Sume of Oxides } & 0.082868 & 0.034144 & 2.43 & 0.1360\end{array}$
Batch $=247$

Bivariate Fit of Li2O By Sum of Oxides

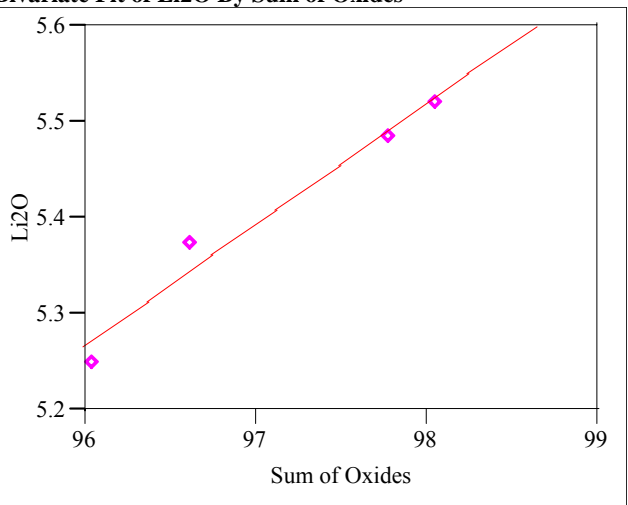

Linear Fit

Linear Fit

$\mathrm{Li} 2 \mathrm{O}=-6.857995+0.1262854$ Sum of Oxides

Summary of Fit

0.969353

$\begin{array}{lr}\text { RSquare Adj } & 0.95403 \\ \text { Root Mean Square Error } & 0.026091\end{array}$

Mean of Response $\quad 5.407547$

Observations (or Sum Wgts)

Analysis of Variance

Source DF Sum of Squares Mean Square F Ratio

$\begin{array}{lllll}\text { Model } & 1 & 0.04306361 & 0.043064 & 63.2593\end{array}$

$\begin{array}{lllll}\text { Error } & 2 & 0.00136149 & 0.000681 & \text { Prob }>\text { F }\end{array}$

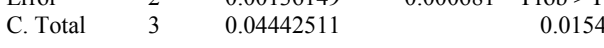

Parameter Estimates

Term Estimate Std Error t Ratio Prob $>\mid t$

$\begin{array}{lllll}\text { Intercept } & -6.857995 & 1.542198 & -4.45 & 0.0470\end{array}$

$\begin{array}{lrrrr}\text { Sum of Oxides } & 0.1262854 & 0.015878 & 7.95 & 0.0154\end{array}$ 
WSRC-TR-2004-00508

Exhibit A10. Relationships between $\mathrm{Li}_{2} \mathrm{O}$ Content and Sum of Oxides of SME Samples
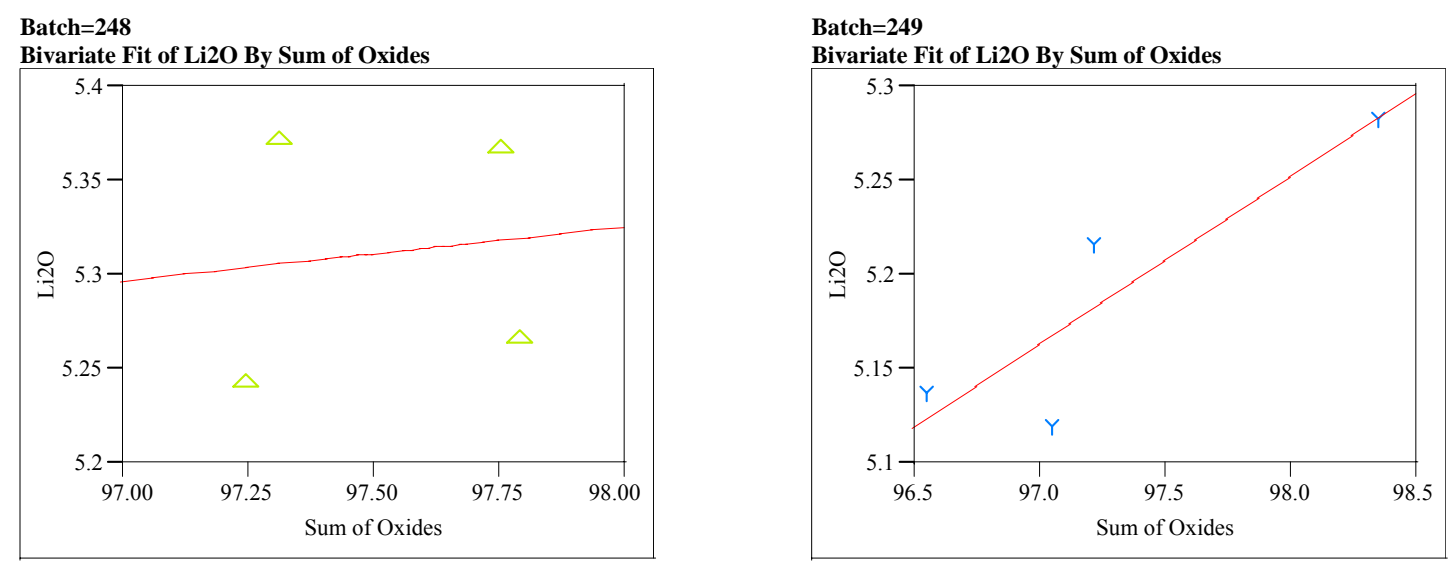

Batch $=250$

Revision 0

\section{Linear Fit \\ Linear Fit}

$\mathrm{Li} 2 \mathrm{O}=2.5046377+0.0287829$ Sum of Oxides

\section{Linear Fit}

Linear Fit

$\mathrm{Li} 2 \mathrm{O}=-3.408634+0.0883675$ Sum of Oxides

Summary of Fit

\section{Summary of Fit}

$\begin{array}{ll}\text { RSquare } & 0.015138 \\ \text { RSquare Adj } & -0.47729 \\ \text { Root Mean Square Error } & 0.081704 \\ \text { Mean of Response } & 5.311743\end{array}$

Mean of Response 5.311743

Analysis of Variance

$\begin{array}{lrrrr}\text { Source } & \text { DF } & \text { Sum of Squares } & \text { Mean Square } & \text { F Ratio } \\ \text { Model } & 1 & 0.00020522 & 0.000205 & 0.0307 \\ \text { Error } & 2 & 0.01335093 & 0.006675 & \text { Prob }>\text { F } \\ \text { C. Total } & 3 & 0.01355615 & & 0.8770\end{array}$

Parameter Estimates

$\begin{array}{lrrrr}\text { Term } & \text { Estimate } & \text { Std Error } & \mathrm{t} \text { Ratio } & \text { Prob }>|t| \\ \text { Intercept } & 2.5046377 & 16.01002 & 0.16 & 0.8900\end{array}$

$\begin{array}{lrrrr}\text { Intercept } & 2.5046377 & 16.01002 & 0.16 & 0.8900 \\ \text { Sum of Oxides } & 0.0287829 & 0.16416 & 0.18 & 0.8770\end{array}$

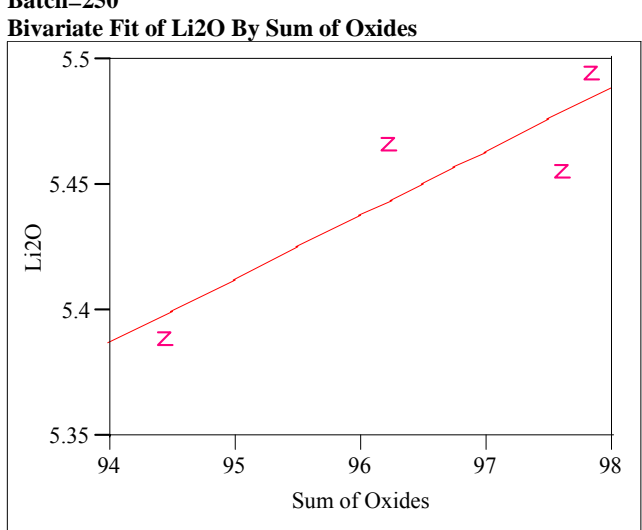

\section{Linear Fit}

Linear Fit

$\mathrm{Li} 2 \mathrm{O}=2.9946469+0.0254487$ Sum of Oxides

Summary of Fit

$\begin{array}{lr}\text { RSquare } & 0.79429 \\ \text { RSquare Adj } & 0.691436 \\ \text { Root Mean Square Error } & 0.024837\end{array}$

Mean of Response 5.451143

Analysis of Variance

Source DF Sum of Squares Mean Square F Ratio

$\begin{array}{llllll}\text { Model } & 1 & 0.00476389 & 0.004764 & 7.7224 \\ \text { Enor } & 2 & 0.00123378 & 0.000617 & \text { Prb }\end{array}$

$\begin{array}{lllrr}\text { Error } & 2 & 0.00123378 & 0.000617 & \text { Prob }>\text { F } \\ \text { C. Total } & 3 & 0.00599766 & & 0.1088\end{array}$

Parameter Estimates

$\begin{array}{lrrrr}\text { Parameter Estimates } & & & & \\ \text { Term } & \text { Estimate } & \text { Std Error } & \mathrm{t} \text { Ratio } & \text { Prob }>|t| \\ \text { Intercept } & 2.9946469 & 0.884059 & 3.39 & 0.0772\end{array}$

$\begin{array}{lllll}\text { Strecpt } & 2.9946469 & 0.884059 & 3.39 & 0.0772\end{array}$ 
WSRC-TR-2004-00508

Exhibit A10. Relationships between $\mathrm{Li}_{2} \mathrm{O}$ Content and Sum of Oxides of SME Samples

Revision 0

Batch $=251$

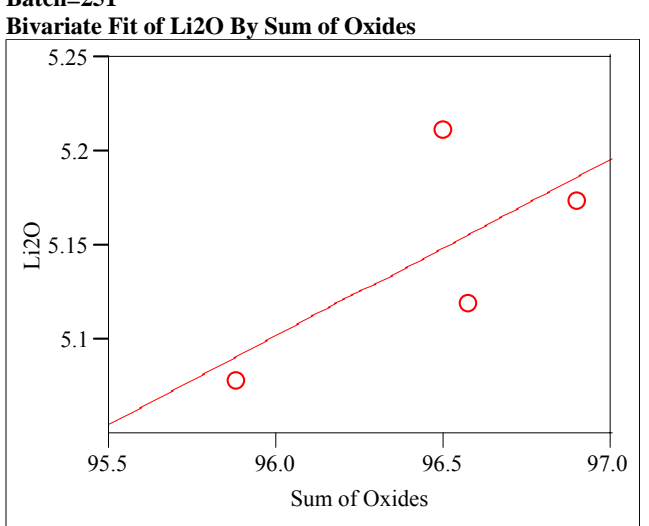

\section{Linear Fit}

Linear Fit

$\mathrm{Li} 2 \mathrm{O}=-3.889327+0.0936614$ Sum of Oxides

\section{Summary of Fit}

$\begin{array}{ll}\text { RSquare } & 0.457718 \\ \text { RSquare Adj } & 0.186578 \\ \text { Root Mean Square Error } & 0.052995 \\ \text { Mean of Response } & 5.145969\end{array}$

Mean of Response

Analysis of Variance

Source DF Sum of Squares Mean Square F Ratio

$\begin{array}{lllll}\text { Model } & 1 & 0.00474106 & 0.004741 & 1.6881\end{array}$

$\begin{array}{lllrr}\text { Error } & 2 & 0.00561696 & 0.002808 & \text { Prob }>\text { F } \\ \text { C. Total } & 3 & 0.01035802 & & 0.3235\end{array}$

Parameter Estimates

$\begin{array}{lrrrr}\text { Term } & \text { Estimate } & \text { Std Error } & \mathrm{t} \text { Ratio } & \text { Prob }>|\mathrm{t}| \\ \text { Intercept } & -3.889327 & 6.954146 & -0.56 & 0.6322 \\ \text { Sum of Oxides } & 0.0936614 & 0.072087 & 1.30 & 0.3235\end{array}$
Batch $=252$

Bivariate Fit of Li2O By Sum of Oxides

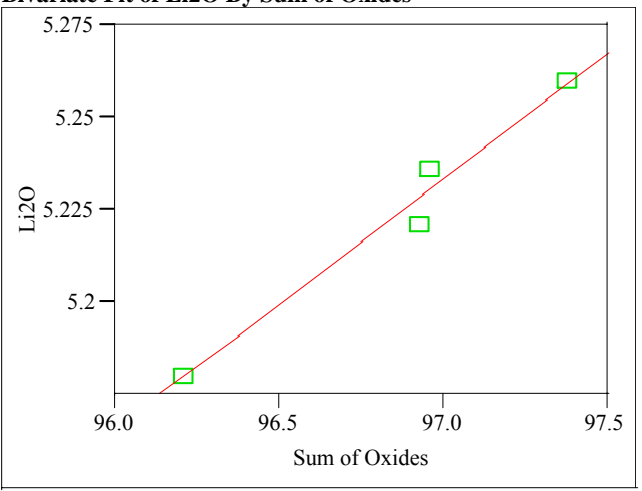

\section{Linear Fit}

Linear Fit

$\mathrm{Li} 2 \mathrm{O}=-1.359193+0.0679639$ Sum of Oxides

Summary of Fit

$\begin{array}{lr}\text { RSquare } & 0.974115 \\ \text { RSquare Adj } & 0.961172 \\ \text { Root Mean Square Error } & 0.006595 \\ \text { Mean of Response } & 5.224012 \\ \text { Observations (or Sum Wgts) } & 4\end{array}$

Observations (or Sum Wgts)

Source DF Sum of Squares Mean Square F Ratio

$\begin{array}{lllll}\text { Model } & 1 & 0.00327338 & 0.003273 & 75.2638\end{array}$

$\begin{array}{lllrr}\text { Error } & 2 & 0.00008698 & 0.000043 & \text { Prob }>\text { F } \\ \text { C. Total } & 3 & 0.00336036 & & 0.0130\end{array}$

Parameter Estimates

Term Estimate Std Error t Ratio Prob $>|t|$

Intercept

$\begin{array}{rrrr}-1.359193 & 0.758837 & -1.79 & 0.2151 \\ 0.0679639 & 0.007834 & 8.68 & 0.0130\end{array}$

$\begin{array}{lllll}\text { Sum of Oxides } & 0.0679639 & 0.007834 & 8.68 & 0.0130\end{array}$
Batch $=253$

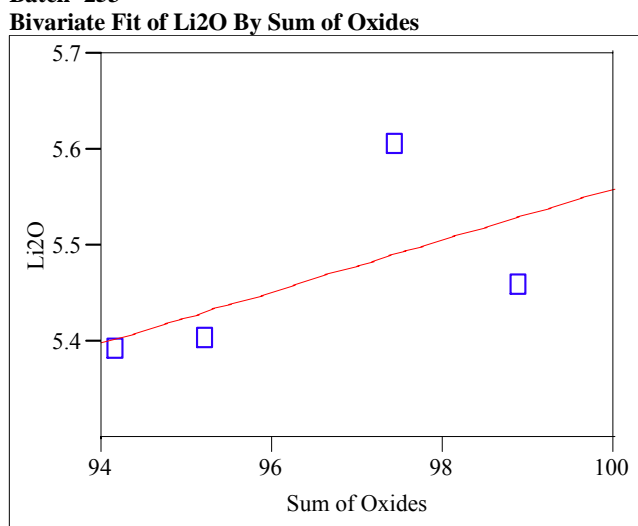

\section{Linear Fit}

Linear Fit

$\mathrm{Li} 2 \mathrm{O}=2.8787888+0.0268065$ Sum of Oxides

Summary of Fit

0.339065

RSquare Adj $\quad 0.008598$

Root Mean Square Error $\quad 0.097692$

$\begin{array}{lr}\text { Mean of Response } & 5.463522 \\ \text { Observations (or Sum Wgts) } & 4\end{array}$

Analysis of Variance

Source DF Sum of Squares Mean Square F Ratio

$\begin{array}{lllll}\text { Model } & 1 & 0.00979200 & 0.009792 & 1.0260\end{array}$

$\begin{array}{lllll}\text { Error } & 2 & 0.01908739 & 0.009544 & \text { Prob }>\text { F }\end{array}$

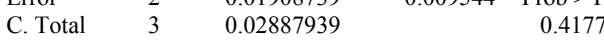

Parameter Estimates

Term Estimate Std Error t Ratio Prob $>\mid t$

$\begin{array}{lllll}\text { Intercept } & 2.8787888 & 2.552219 & 1.13 & 0.3765 \\ \text { Sum of Oxides } & 0.0268065 & 0.026464 & 1.01 & 0.4177\end{array}$ 
WSRC-TR-2004-00508

Exhibit A10. Relationships between $\mathrm{Li}_{2} \mathrm{O}$ Content and Sum of Oxides of SME Samples

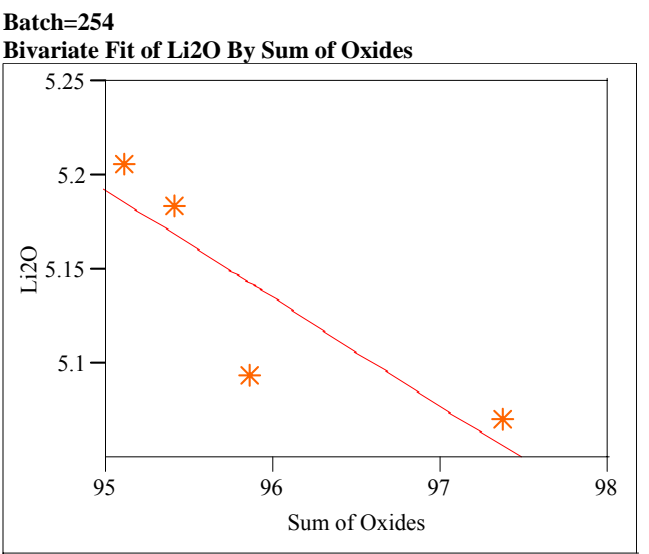

\section{Linear Fit}

Linear Fit

$\mathrm{i} 2 \mathrm{O}=10.661639-0.0575656$ Sum of Oxides

Summary of Fit

\begin{tabular}{|c|c|c|c|c|c|}
\hline \multicolumn{3}{|c|}{ RSquare } & \multicolumn{3}{|c|}{0.75514} \\
\hline \multicolumn{3}{|c|}{$\begin{array}{l}\text { RSquare Adj } \\
\text { Root Mean Square Frror }\end{array}$} & \multicolumn{3}{|c|}{0.63271} \\
\hline \multicolumn{3}{|c|}{ Root Mean Square Error } & \multicolumn{3}{|c|}{0.040333} \\
\hline \multicolumn{3}{|c|}{ Mean of Response } & \multicolumn{3}{|c|}{5.138434} \\
\hline \multicolumn{3}{|c|}{ Observations (or Sum Wgts) } & 4 & & \\
\hline \multicolumn{6}{|c|}{ Analysis of Variance } \\
\hline Source & $\mathrm{DF}$ & Sum of Squares & Mean Square & \\
\hline Model & 1 & 0.01003379 & 0.010034 & & \\
\hline Error & 2 & 0.00325353 & 0.001627 & \multicolumn{2}{|c|}{ Prob $>$ F } \\
\hline C. Total & 3 & 0.01328732 & & & \\
\hline \multicolumn{6}{|c|}{ Parameter Estimates } \\
\hline \multicolumn{3}{|l|}{ Term } & Std Error & t Ratio & Prob $>|t|$ \\
\hline \multicolumn{2}{|l|}{ Intercept } & 10.661639 & 2.224021 & 4.79 & 0.0409 \\
\hline \multicolumn{2}{|c|}{ Sum of Oxides } & -0.057566 & 0.023179 & -2.48 & 0.1310 \\
\hline
\end{tabular}

Batch=255

Bivariate Fit of Li2O By Sum of Oxides

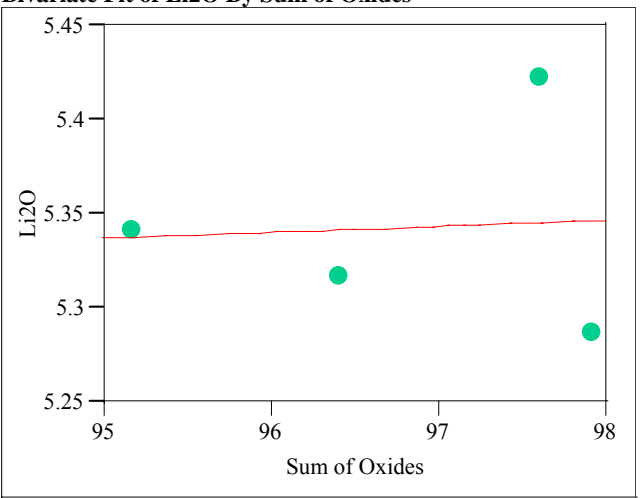

\section{Linear Fit}

Linear Fit

$\mathrm{Li2O}=5.0346382+0.0031804$ Sum of Oxides

Summary of Fit

RSquare

RSquare Adj

Root Mean Square Eror

Mean of Response

0.04924

0.071057

(or Sum Wgts)

5
4

Analysis of Variance

Source DF Sum of Squares Mean Square F Ratio

\begin{tabular}{lllll} 
Model & 1 & 0.00004772 & 0.000048 & 0.0095 \\
\hline & 2 & 0.0100925 & 0.00504 & 0.9314
\end{tabular}

$\begin{array}{lllrr}\text { Error } & 2 & 0.01009825 & 0.005049 & \text { Prob }>\text { F } \\ \text { C. Total } & 3 & 0.01014597 & & 0.9314\end{array}$

Parameter Estimates

$\begin{array}{lrrrr}\text { Parameter Estimates } & & & & \\ \text { Term } & \text { Estimate } & \text { Std Error } & \mathrm{t} \text { Ratio } & \text { Prob }>|\mathrm{t}| \\ \text { Intercept } & 5.0346382 & 3.166063 & 1.59 & 0.2528 \\ \text { Sum of } & 0.0031804 & 0.032714 & 0.10 & 0.9314\end{array}$
Batch=256

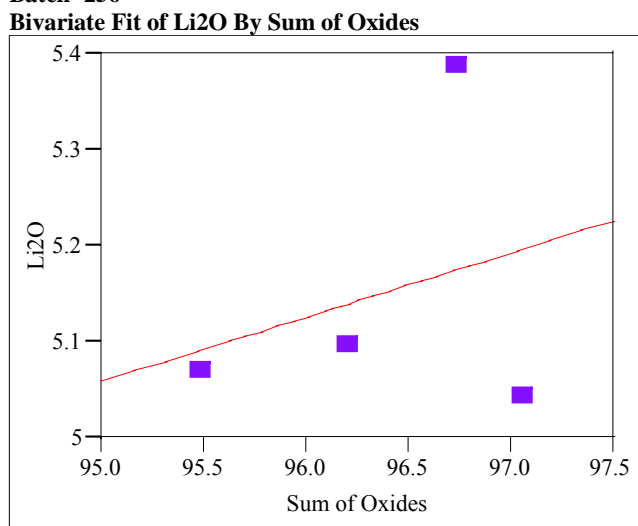

Linear Fit

Linear Fit

$\mathrm{Li} 2 \mathrm{O}=-1.313879+0.067078$ Sum of Oxides

Summary of Fit

RSquare Adj $\quad-0.37609$

Root Mean Square Error $\quad 0.188518$

$\begin{array}{lr}\text { Mean of Response } & 5.149737 \\ \text { Observations (or Sum Wgts) } & 4\end{array}$

Analysis of Variance

Source DF Sum of Squares Mean Square F Ratio

$\begin{array}{lllll}\text { Model } & 1 & 0.00640033 & 0.006400 & 0.1801\end{array}$

$\begin{array}{lllll}\text { Error } & 2 & 0.07107797 & 0.035539 & \text { Prob }>\text { F } \\ \text { C. } & 3 & 0.07747830 & & 0.712\end{array}$

$\begin{array}{lllr}\text { C. Total } & 3 & 0.07747830 & 0.03539\end{array}$

Parameter Estimates

Term Estimate Std Error $t$ Ratio Prob $>|t|$

$\begin{array}{lllll}\text { Intercept } & -1.313879 & 15.23124 & -0.09 & 0.9391\end{array}$

$\begin{array}{lrrrr}\text { Sum of Oxides } & -1.313879 & 15.23124 & -0.09 & 0.9391 \\ & 0.067078 & 0.158063 & 0.42 & 0.7126\end{array}$ 
WSRC-TR-2004-00508

Exhibit A10. Relationships between $\mathrm{Li}_{2} \mathrm{O}$ Content and Sum of Oxides of SME Samples

Revision 0

Batch $=257$

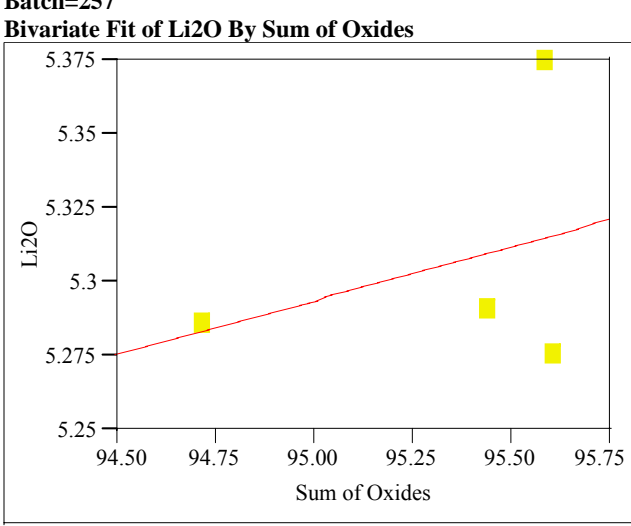

Linear Fit

Linear Fit

$\mathrm{Li} 2 \mathrm{O}=1.8205285+0.0365572$ Sum of Oxides

\section{Summary of Fit}

RSquare

RSquare Adj

Root Mean Square Error

Mean of Response

\subsection{7}

-0.32863
0.052622

5.305822

Observations (or Sum Wgts)

Analysis of Variance

Source DF Sum of Squares Mean Square F Ratio

$\begin{array}{lrrrr}\text { Model } & 1 & 0.00071434 & 0.000714 & 0.2580\end{array}$

$\begin{array}{lllrr}\text { Error } & 2 & 0.00553825 & 0.002769 & \text { Prob }>\text { F } \\ \text { C. Total } & 3 & 0.00625259 & 0.6620\end{array}$

Parameter Estimates

Term

Estimate Std Error $\mathrm{t}$ Ratio Prob $>|\mathrm{t}|$

Sum of Oxides

$\begin{array}{rrrr}\text { Estimate } & \text { Std Error } & \text { t Ratio } & \text { Prob }>|t| \\ .8205285 & 6.862171 & 0.27 & 0.8156\end{array}$ $\begin{array}{llll}1.8205285 & 6.862171 & 0.27 & 0.8156 \\ 0.0365572 & 0.071977 & 0.51 & 0.6620\end{array}$
Batch $=258$

Bivariate Fit of Li2O By Sum of Oxides

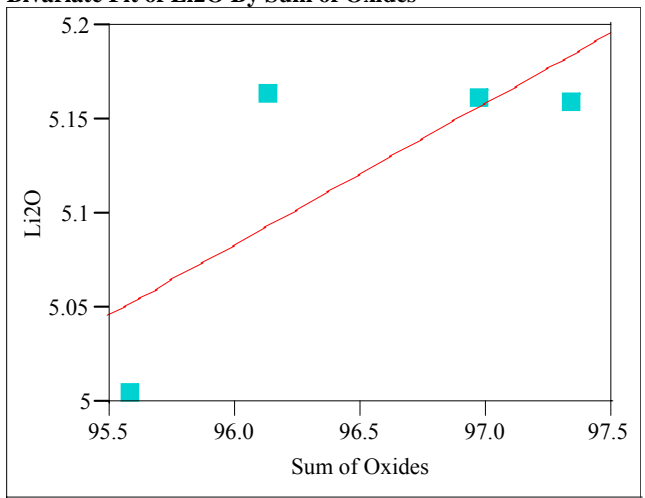

\section{Linear Fit}

Linear Fit

$\mathrm{Li} 2 \mathrm{O}=-2.122348+0.0750586$ Sum of Oxides

Summary of Fit

RSquare

RSquare Adj

Root Mean Square

0.576473

0.062649

5.121211

Observations (or Sum Wgts)

Analysis of Variance

Source DF Sum of Squares Mean Square F Ratio

$\begin{array}{lllll}\text { Model } & 1 & 0.01068443 & 0.010684 & 2.7223 \\ & 2 & 0.00784969 & 0.003925 & \text { P.b }\end{array}$

$\begin{array}{lllrr}\text { Error } & 2 & 0.00784969 & 0.003925 & \text { Prob }>\text { F } \\ \text { C. Total } & 3 & 0.01853412 & & 0.2407\end{array}$

Parameter Estimates

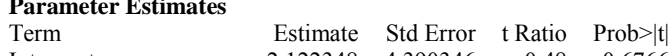

$\begin{array}{lrllll}\text { Intercept } & -2.122348 & 4.390346 & -0.48 & 0.6766\end{array}$
Batch=259

Bivariate Fit of Li2O By Sum of Oxides

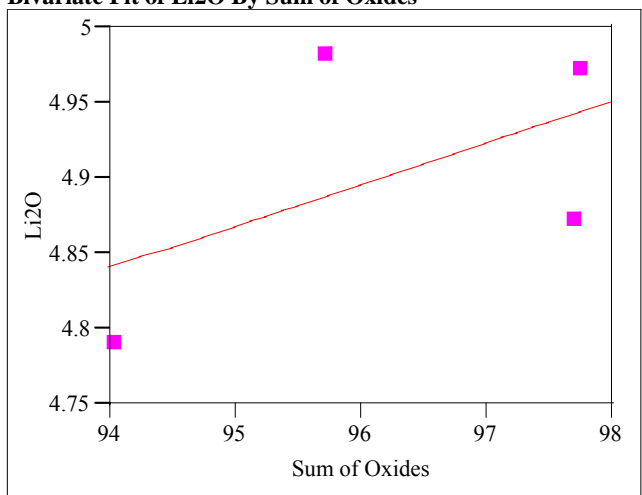

\section{Linear Fit}

Linear Fit

$\mathrm{Li} 2 \mathrm{O}=2.2337289+0.0277295$ Sum of Oxides

Summary of Fit

RSquare

$\begin{array}{ll}0.29651 & -0.05052 \\ \text { Root Mean Square Error } & 0.092667\end{array}$

Roon of Response $\quad 0.092667$

4.903768

Analysis of Variance

Source DF Sum of Squares Mean Square F Ratio

$\begin{array}{lllll}\text { Model } & \quad & 0.00734819 & 0.007348 & 0.8557\end{array}$

$\begin{array}{lllll}\text { Error } & 2 & 0.01717432 & 0.008587 & \text { Prob }>\text { F } \\ \text { C. Total } & 3 & 0.02452251 & & 0.452\end{array}$

Parameter Estimates

Term Estimate Std Error t Ratio Prob $>\mid t$

$\begin{array}{lrrrr}\text { Intercept } & \text { Estimate } & \text { Std Error } & & \\ \text { Inatio } & \text { Prob }>\mid t\end{array}$

$\begin{array}{llllll}\text { Sum of Oxides } & 0.0277295 & 0.029976 & 0.93 & 0.4526\end{array}$ 
WSRC-TR-2004-00508

Exhibit A10. Relationships between $\mathrm{Li}_{2} \mathrm{O}$ Content and Sum of Oxides of SME Samples

Revision 0

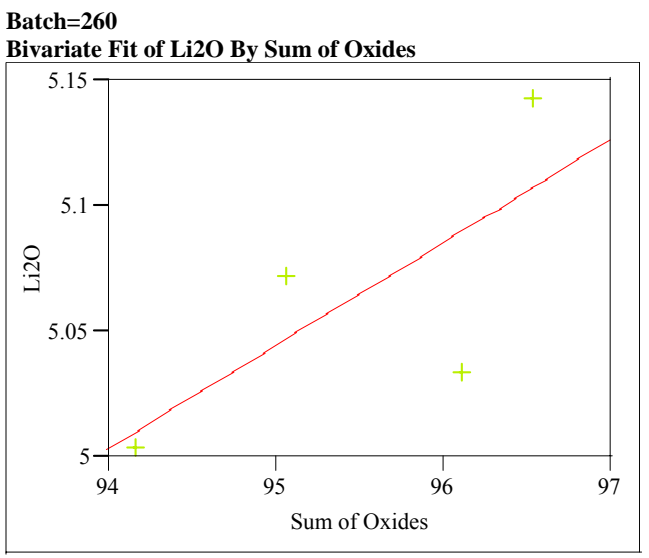

\section{Linear Fit \\ Linear Fit}

$\mathrm{Li} 2 \mathrm{O}=1.1498706+0.0409917$ Sum of Oxides

\section{Summary of Fit}

\begin{tabular}{|c|c|c|c|c|c|}
\hline \multirow{2}{*}{\multicolumn{3}{|c|}{ RSquare }} & \multicolumn{3}{|l|}{.528325} \\
\hline \multirow{2}{*}{\multicolumn{2}{|c|}{ Root Mean Square Error }} & & \multicolumn{3}{|c|}{0.292488} \\
\hline & & Root Mean Square Error & \multicolumn{3}{|c|}{0.050842} \\
\hline \multicolumn{3}{|c|}{ Mean of Response } & \multicolumn{3}{|l|}{063083} \\
\hline \multicolumn{3}{|c|}{ Observations (or Sum Wgts) } & \multicolumn{3}{|l|}{4} \\
\hline \multicolumn{6}{|c|}{ Analysis of Variance } \\
\hline Source & $\mathrm{DF}$ & Sum of Squares & Mean Square & \multicolumn{2}{|c|}{ F Ratio } \\
\hline Model & 1 & 0.00579074 & 0.005791 & \multicolumn{2}{|c|}{2.2402} \\
\hline Error & 2 & 0.00516982 & 0.002585 & \multicolumn{2}{|c|}{ Prob $>$ F } \\
\hline C. Total & 3 & 0.01096057 & & \multicolumn{2}{|c|}{0.2731} \\
\hline \multicolumn{6}{|c|}{ Parameter Estimates } \\
\hline \multicolumn{3}{|c|}{ Term } & Std Error & t Ratio & $\operatorname{Prob}>|t|$ \\
\hline Intercept & & 1.14987 & $6 \quad 2.614627$ & 0.44 & 0.70 \\
\hline Sum of O & ides & 0.04099 & $\begin{array}{ll}7 & 0.027387\end{array}$ & 1.50 & 0.2731 \\
\hline
\end{tabular}

Batch=261

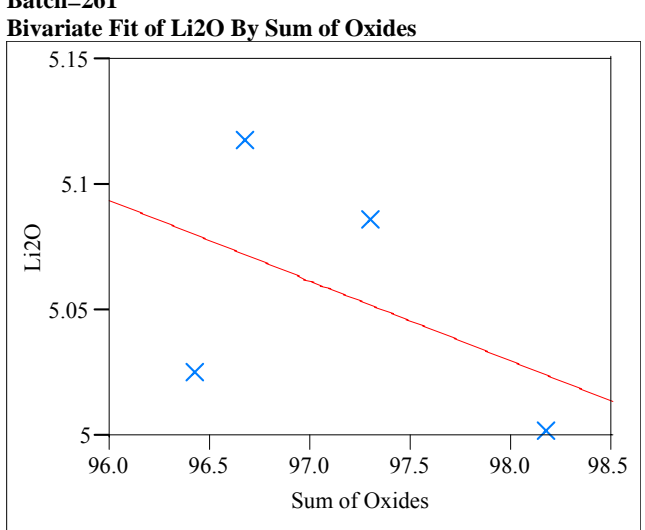

\section{Linear Fit}

Linear Fit

$\mathrm{Li} 2 \mathrm{O}=8.1612185-0.031954$ Sum of Oxides

Summary of Fit

$\begin{array}{lr}\text { RSquare } & 0.215561 \\ \text { Rquare Adj } & -0.17666 \\ \text { Root Mean Square Error } & 0.058055 \\ \text { Mean of Response } & 5.057162 \\ \text { Observations (or Sum Wgts) } & 4\end{array}$

Observations (or Sum Wgts)

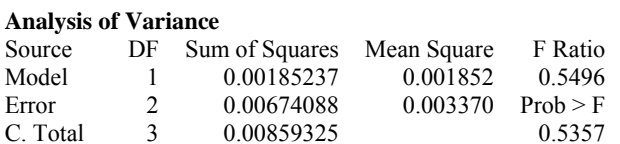

\begin{tabular}{lllll} 
Error & 2 & 0.00674088 & 0.003370 & 0.5496 \\
\hline C. Tota & 3 & 0.0085325 & 0.5357
\end{tabular}

$\begin{array}{lll}\text { C. Total } & 0.00859325 & 0.5357\end{array}$

Parameter Estimates

Term Estimate Std Error t Ratio Prob $>|t|$

$\begin{array}{lllll}\text { Intercept } & 8.1612185 & 4.187158 & 1.95 & 0.1906\end{array}$
Batch=262

Bivariate Fit of Li2O By Sum of Oxides

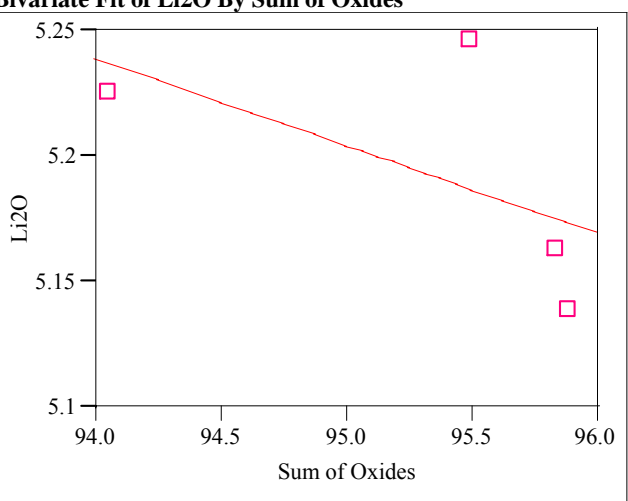

\section{Linear Fit}

Linear Fit

$\mathrm{Li} 2 \mathrm{O}=8.521486-0.0349198$ Sum of Oxides

Summary of Fit

$\begin{array}{ll}\text { RSquare } & 0.351198 \\ \text { RSquare Adj } & 0.026796\end{array}$

RSquare Adj $\quad 0.026796$

Rean of Response $\quad 0.05012$

Observations (or Sum Wgts) 5.19333

Analysis of Variance

Source DF Sum of Squares Mean Square F Ratio

$\begin{array}{lllll}\text { Model } & 1 & 0.00271964 & 0.002720 & 1.0826\end{array}$

$\begin{array}{lllrr}\text { Error } & 2 & 0.00502425 & 0.002512 & \text { Prob > F } \\ \text { C. Total } & 3 & 0.00774389 & & 0.4074\end{array}$

Parameter Estimates

Term $\quad$ Estimate Std Error $t$ Ratio Prob $>|t|$

$\begin{array}{lllrr}\text { Intercept } & 8.521486 & 3.198763 & 2.66 & 0.1167\end{array}$ 
WSRC-TR-2004-00508

Exhibit A10. Relationships between $\mathrm{Li}_{2} \mathrm{O}$ Content and Sum of Oxides of SME Samples

Revision 0

Batch=263

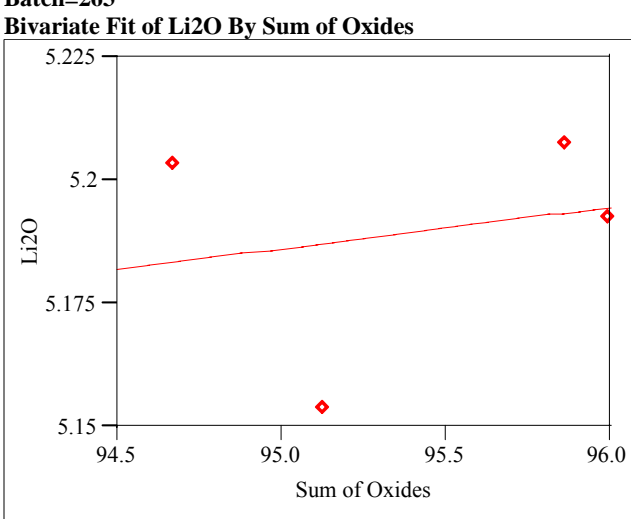

Linear Fit

Linear Fit

$\mathrm{Li} 2 \mathrm{O}=4.3854703+0.0084274$ Sum of Oxides

\section{Summary of Fit}

$\begin{array}{lr}\text { RSquare } & 0.046186 \\ \text { RSquare Adj } & -0.43072 \\ \text { Root Mean Square Error } & 0.029323 \\ \text { Mean of Response } & 5.189565 \\ \text { Observations (or Sum Wgts) } & 4\end{array}$

Observations (or Sum Wgts)

DF Sum of Squares Mean Square F Ratio

$\begin{array}{lllll}\text { Error } & 2 & 0.00171973 & 0.000860 & \text { Prob }>\text { F } \\ \text { C. } & 3 & 0.00180301 & & 0.785\end{array}$

\section{Parameter Estimates}

$\begin{array}{lrrrr}\text { Intercept } & 4.3854703 & 2.583916 & 1.70 & 0.2317 \\ \text { Sum of Oxides } & 0.0084274 & 0.02708 & 0.31 & 0.7851\end{array}$

$\begin{array}{rrrr}\text { Estimate } & \text { Std Error } & t \text { Ratio } & \text { Prob }>|t|\end{array}$
Batch $=264$

Bivariate Fit of Li2O By Sum of Oxides

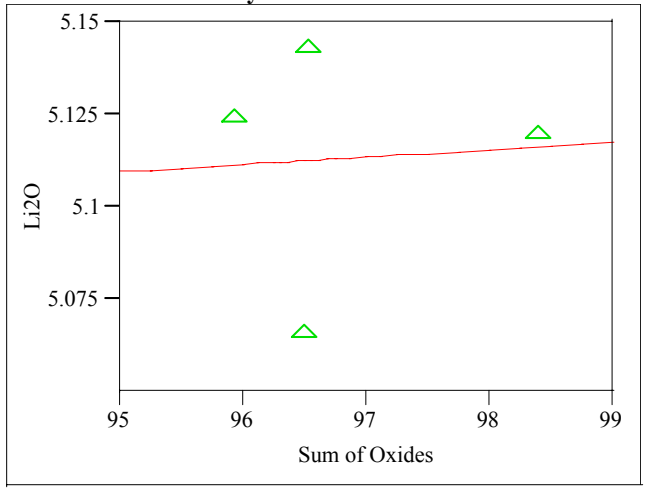

\section{Linear Fit}

Linear Fit

$\mathrm{Li} 2 \mathrm{O}=4.9223189+0.0019702$ Sum of Oxides

Summary of Fit

$\begin{array}{lr}\text { RSquare } & 0.004083 \\ \text { RSquare Adj } & -0.49387 \\ \text { Root Mean Square Error } & 0.040595 \\ \text { Mean of Response } & 5.113138 \\ \text { Observations (or Sum Wgts) } & 4\end{array}$

Observations (or Sum Wgts)

Analysis of Variance

Source DF Sum of Squares Mean Square F Ratio

$\begin{array}{lrrrr}\text { Model } & 1 & 0.00001351 & 0.000014 & 0.0082\end{array}$

$\begin{array}{llllll}\text { Error } & 2 & 0.00329586 & 0.001648 & \text { Prob > F }\end{array}$

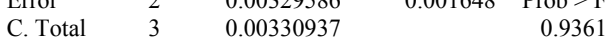

Parameter Estimates

Term Estimate Std Error $\quad \mathrm{t}$ Ratio Prob $>|\mathrm{t}|$ $\begin{array}{lllrr}\text { Intercept } & 4.9223189 & 2.107297 & 2.34 & 0.1446 \\ \text { Sum } & 0.0019702 & 0.021757 & 0.09 & 0.9361\end{array}$
Batch $=265$

Bivariate Fit of Li2O By Sum of Oxides

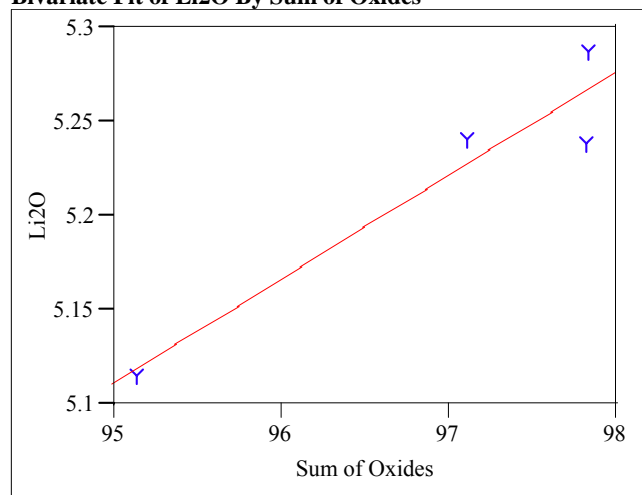

\section{Linear Fit}

Linear Fit

$\mathrm{Li2O}=-0.129792+0.0551634$ Sum of Oxides

Summary of Fit

$\begin{array}{lr}\text { RSquare } & 0.912886 \\ \text { RSquare Adj } & 0.869329 \\ \text { Root Mean Square Error } & 0.026608 \\ \text { Mean of Response } & 5.220244 \\ \text { Observations (or Sum Wgts) } & 4\end{array}$

Analysis of Variance

Source DF Sum of Squares Mean Square F Ratio

$\begin{array}{lllll}\text { Model } & 1 & 0.01483779 & 0.014838 & 20.9584\end{array}$

$\begin{array}{lllll}\text { Error } & 2 & 0.00141592 & 0.000708 & \text { Prob }>\text { F } \\ \text { C. Total } & 3 & 0.01625371 & & 0.0445\end{array}$

Parameter Estimates

Term $\quad$ Estimate Std Error t Ratio Prob $>|t|$

$\begin{array}{lrrrr}\text { Intercept } & -0.129792 & 1.168706 & -0.11 & 0.9217\end{array}$

Sum of Oxides 